\title{
The hypoxic tumor microenvironment: from imaging to targeting
}

Citation for published version (APA):

Peeters, S. G. J. A. (2015). The hypoxic tumor microenvironment: from imaging to targeting. [Doctoral Thesis, Maastricht University]. Datawyse / Universitaire Pers Maastricht. https://doi.org/10.26481/dis.20150709sp

Document status and date:

Published: 01/01/2015

DOI:

10.26481/dis.20150709sp

Document Version:

Publisher's PDF, also known as Version of record

\section{Please check the document version of this publication:}

- A submitted manuscript is the version of the article upon submission and before peer-review. There can be important differences between the submitted version and the official published version of record.

People interested in the research are advised to contact the author for the final version of the publication, or visit the DOI to the publisher's website.

- The final author version and the galley proof are versions of the publication after peer review.

- The final published version features the final layout of the paper including the volume, issue and page numbers.

Link to publication

\footnotetext{
General rights rights.

- You may freely distribute the URL identifying the publication in the public portal. please follow below link for the End User Agreement:

www.umlib.nl/taverne-license

Take down policy

If you believe that this document breaches copyright please contact us at:

repository@maastrichtuniversity.nl

providing details and we will investigate your claim.
}

Copyright and moral rights for the publications made accessible in the public portal are retained by the authors and/or other copyright owners and it is a condition of accessing publications that users recognise and abide by the legal requirements associated with these

- Users may download and print one copy of any publication from the public portal for the purpose of private study or research.

- You may not further distribute the material or use it for any profit-making activity or commercial gain

If the publication is distributed under the terms of Article $25 \mathrm{fa}$ of the Dutch Copyright Act, indicated by the "Taverne" license above, 
a

ه The hypoxic tumor microenvironment: from imaging to targeting

$\Delta$

$\Delta$

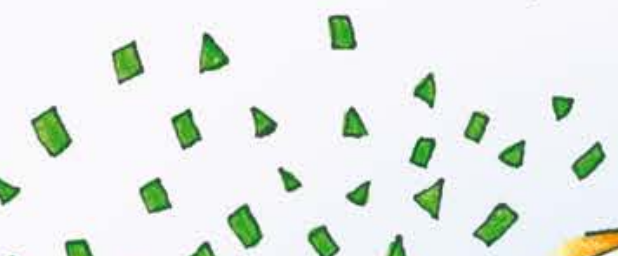

$\Delta: \Delta \Delta, \triangle \Delta$

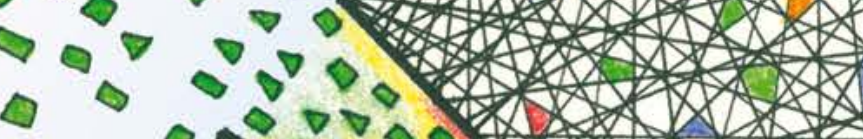
\begin{tabular}{lll}
$\triangle$ & \\
\hline
\end{tabular}

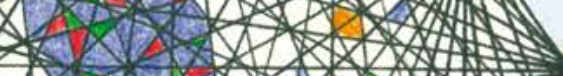
1218 111 121

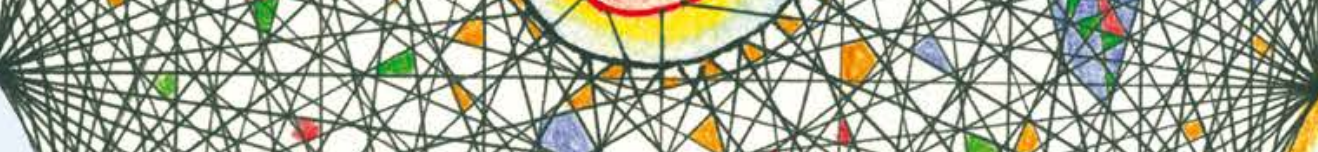
1. 1 1 1. 1 1

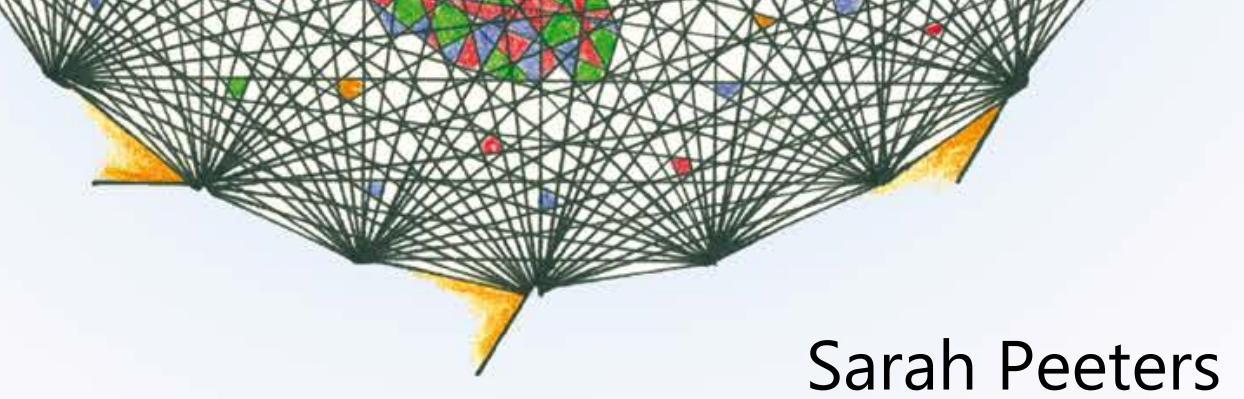




\section{Cover}

Abstract and free interpretation of imaging and targeting of the hypoxic tumor microenvironment

Cover design: Ciel Peeters - Hermans

Layout: $\quad$ Sarah Peeters

\section{Production}

Printing: $\quad$ Datawyse | Universitaire Pers Maastricht

ISBN: 9789461594501

(c) Sarah G.J.A. Peeters, Maastricht 2015. 


\title{
The hypoxic tumor microenvironment: from imaging to targeting
}

\author{
Proefschrift \\ ter verkrijging van de graad van doctor aan de Universiteit Maastricht, \\ op gezag van de Rector Magnificus, Prof dr. L.L.G. Soete, \\ volgens het besluit van het College van Decanen \\ in het openbaar te verdedigen \\ op donderdag 9 juli 2015 om 12.00 uur \\ door \\ Sarah Gertruda Johanna Adriana Peeters
}

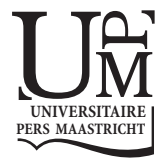




\section{Promotor}

Prof. dr. P. Lambin

\section{Co-promotor}

Dr. L.J. Dubois

\section{Beoordelingscommissie}

Prof. dr. F.C.S. Ramaekers (voorzitter)

Prof. dr. R.P. Coppes, UMC Groningen

Prof. dr. M.J. Daemen, AMC Amsterdam

Prof. dr. F. Mottaghy

Dr. K.M.A. Rouschop 


\section{Contents}

\section{Introduction}

Chapter 1 Introduction and outline of the thesis

Part I: Noninvasive imaging of the hypoxic tumor microenvironment

Chapter 2 Current preclinical and clinical applications of hypoxia PET imaging

Chapter 3 Comparison of 3 hypoxia PET tracers

Chapter $4 \quad\left[{ }^{18} \mathrm{~F}\right] \mathrm{VM} 4-037$ tumor imaging

Part II: Targeting of the hypoxic tumor regions and the hypoxia response

Chapter 5 TH-302 and radiotherapy monitored by $\left[{ }^{18} \mathrm{~F}\right] \mathrm{HX} 4$ imaging 85

$\begin{array}{lll}\text { Chapter } 6 & \text { CAIX inhibition and radiotherapy } & 109\end{array}$

$\begin{array}{lll}\text { Chapter } 7 & \text { CAIX dual targeting enhances effect IR } & 131\end{array}$

\section{Discussion}

Chapter 8 General discussion \& Future perspectives

Summary

Samenvatting

Valorization addendum

Acknowledgements / Dankwoord

Curriculum Vitae

List of Publications 

Chapter 1

Introduction and outline of the thesis 


\section{Cancer}

Cancer is a leading cause of death worldwide. It was estimated that there would be 3.45 million new cases of cancer and 1.75 million cancer related deaths in 2012 only in Europe. In that year, the most common cancer sites for male patients were prostate, lung and colorectum while for female patients this was breast, colorectum and lung. The highest overall mortality rates were observed for lung, colorectum, breast and stomach (1).

Cancer is characterized by a mass of cells with an abnormal behavior, rapidly dividing and the potential to invade other tissues of the body. In normal healthy tissue, cells are replaced once they become old or damaged. During the lifetime of the cell, however, mutations can be introduced to the genetic material (DNA), which can be inherited by the daughter cells. When multiple mutations occur sequentially this can lead to an altered cell function, e.g. uncontrolled cell growth and evasion of apoptosis (2). When these cells are not cleared by the surrounding tissue, a mass of superfluous cells can arise, called a benign cancer, which can become malignant when the cancer is more aggressive and has the potential to invade the surrounding tissues. Moreover, a tumor is able to provide itself with sufficient nutrition and oxygen by inducing the development of new blood vessels. All these tumor characteristics are called the hallmarks of cancer and have been extensively described by Hanahan and Weinberg (3).

The treatment of a diagnosed cancer patient generally consists of a combination of chemotherapy, radiotherapy or surgery (4). However, these therapies lose their efficacy in the presence of regions with low oxygen concentrations, hypoxia, in the tumor $(5,6)$. Therefore, knowledge about hypoxia and its downstream pathways, the hypoxia response, is important. Moreover visualization of hypoxia will provide insights in therapy outcome and gives the ability to adapt therapy. Additionally, new drugs are needed, directed specifically against those therapy-resistance regions, to complement current treatments.

\section{Hypoxia and hypoxia response}

Development of new blood vessels (angiogenesis) is of great importance for the growth of tumor cells. To stimulate the development of blood vessel angiogenic signals are secreted by the tumor itself. However due to the rapid tumor growth vessels are 
often leaky and chaotically structured. Blood supply to the tumor is therefore hampered, leading to scarcity of nutrition and oxygen (hypoxia). Different kinds of hypoxia, acute, chronic and cycling hypoxia, are known to arise. Acute perfusionlimited hypoxia occurs when existing vessels collapse or are temporally obstructed preventing blood flow to a certain region. Chronic diffusion-limited hypoxia is characterized by the outgrowth of tumor cells from the vessels, leading to an oxygen gradient in which oxygen does not reach all the cells surrounding a blood vessel (7). When a region of tumor cells is exposed to oxygen deprivation alternated with periods of reoxygenation this is referred to as cycling hypoxia. This is recognized as a separate status of the tumor since the behavior of those cells can be completely different from well oxygenated or hypoxic cells $(8,9)$.

Hypoxic conditions are known to reduce the efficacy of radiotherapy. The basic principle of ionizing radiation is to damage the cancer cell to such an extent that they will die. Free radicals are formed that can induce damage immediately to the DNA. Indirect damaging occurs when in or close to the DNA radicals $(\mathrm{OH})$ are formed from ionizing radiation in combination with water $\left(\mathrm{H}_{2} \mathrm{O}\right)$. In the presence of oxygen, the damage is fixed. However, when oxygen concentrations are low, the radical interacts with a proton and forms water again. It has been shown that a 2.5 to 3 fold higher radiation dose is required in hypoxic areas to obtain the same radiation response as in well-oxygenated regions (7).

When a cell is exposed to low oxygen concentrations it induces changes in order to survive (10). One of the main changes occurring rapidly after a drop in oxygen is stabilization of the Hypoxia inducible factor 1 alpha (HIF-1 $\alpha$ ). Whereas under normal oxygen concentrations HIF- $1 \alpha$ is covalently modified by prolyl hydroxylases facilitating the interaction with the Von Hippel-Lindau (VHL) complex and inducing constant degradation via ubiquitination, low oxygen concentrations stabilize HIF-1 $\alpha$ enabling the interaction with HIF-1 $\beta$ (also known as 'aryl hydrocarbon receptor nuclear translocator' [ARNT]) and the translocation to the nucleus. The formed HIF complex recognizes and binds to the 'hypoxia-response-elements' (HRE), a specific base sequence on the DNA, located in the promotor region of some genes. This initiates together with cofactors, such as CBP/p300 and the DNA polymerase II (Pol II) complex, the transcription of hypoxia response genes, among others VEGF (vascular endothelial growth factor), GLUT-1 (glucose transporter) and CAIX (Carbonic Anhydrase IX) (11). 
As a consequence of the low oxygen concentrations cells can no longer rely on the oxidative phosphorylation for the production of their energy (ATP). Therefore cells have to use the anaerobic glycolysis, which is less effective in the production of ATP. However, by the HIF dependent upregulation of GLUT-1, more glucose is transported into the cell providing the cell with intermediary metabolites that are needed for the growth and maintenance of cells under stress conditions (11). The anaerobic glycolysis generates the waste product lactate that accumulates in the cytoplasm, acidifying the intracellular $\mathrm{pH}$. This unfavorable situation is counteracted by an increased expression and activity of plasma membrane transporters, like sodium-proton exchanger 1 (NHE) and monocarboxylate transporters (MCT) or other $\mathrm{pH}$ regulating proteins like CAIX. While NHE and MCT directly export acidic products, CAIX catalyzes the conversion of carbon dioxide $\left(\mathrm{CO}_{2}\right.$, which diffuses passively over the cell membrane) and water $\left(\mathrm{H}_{2} \mathrm{O}\right)$ to bicarbonate $\left(\mathrm{HCO}_{3}{ }^{-}\right)$and a proton $\left(\mathrm{H}^{+}\right)(12)$. Bicarbonate is transported back into the cell by a sodium-bicarbonate co-transporter (NBC) or an anion exchanger (AE) and function as a buffer in maintaining a stable physiological intracellular $\mathrm{pH}$, while the proton remains in the extracellular environment, contributing to the acidification (13) (figure 1.1).

These changes, induced by hypoxia, result in a reversed $\mathrm{pH}$ gradient across the cells. In order to maintain a stable intracellular $\mathrm{pH}(\mathrm{pHi})$ around 7.2 , the extracellular $\mathrm{pH}(\mathrm{pHe})$ is acidified. In normal healthy tissues the pHe is around 7.4 while in hypoxic cancer cells this can be reduced ranging from 7.1 to even 6.7. Besides the reduced radiotherapy efficacy caused by the low availability of oxygen, this reversed $\mathrm{pH}$ gradient over the cell can counteract the function of both radiotherapy and chemotherapeutics (12).

\section{Monitoring tumor hypoxia}

Since hypoxia is known to cause therapy resistance, knowledge about the oxygen status of the tumor before starting treatment is of great value. One way of monitoring tumor hypoxia is using Positron Emission Tomography (PET) imaging, possibly combined with Computed Tomography (CT) for anatomical information. Most PET tracers directly monitoring hypoxia are based on 2-nitroimidazoles that undergo an electron reduction upon low oxygen concentrations followed by trapping in the cell $(15,16)$. Over the last decade several PET tracers have been developed and studied in both preclinical and clinical setting, with the most potent ones being [ $\left.{ }^{18} \mathrm{~F}\right] \mathrm{FMISO}$, $\left[{ }^{18} \mathrm{~F}\right] \mathrm{FAZA}$ and $\left[{ }^{18} \mathrm{~F}\right] \mathrm{HX} 4(17-19)$. 
Another approach is to visualize proteins that are regulated by hypoxia and can be used as a surrogate marker for hypoxia. A very attractive target for this is CAIX since its expression is associated with worse clinical outcome (20). This transmembrane protein is easy accessible since it consists of two extracellular located domains each containing a catalytic active site. Moreover, the expression of CAIX in normal tissues is limited; only stomach, gut and gallbladder epithelia are known to express low levels of this protein (21). Both the extracellular location of the protein and the almost exclusive expression in hypoxic cancer tissues make CAIX an attractive target for both imaging and targeting and this has been investigated by many using either antibodies or small molecule based probes (22). For imaging purposes, antibodies directed against CAIX have been developed and coupled to a radioactive tracer to make them detectable for PET imaging (22).

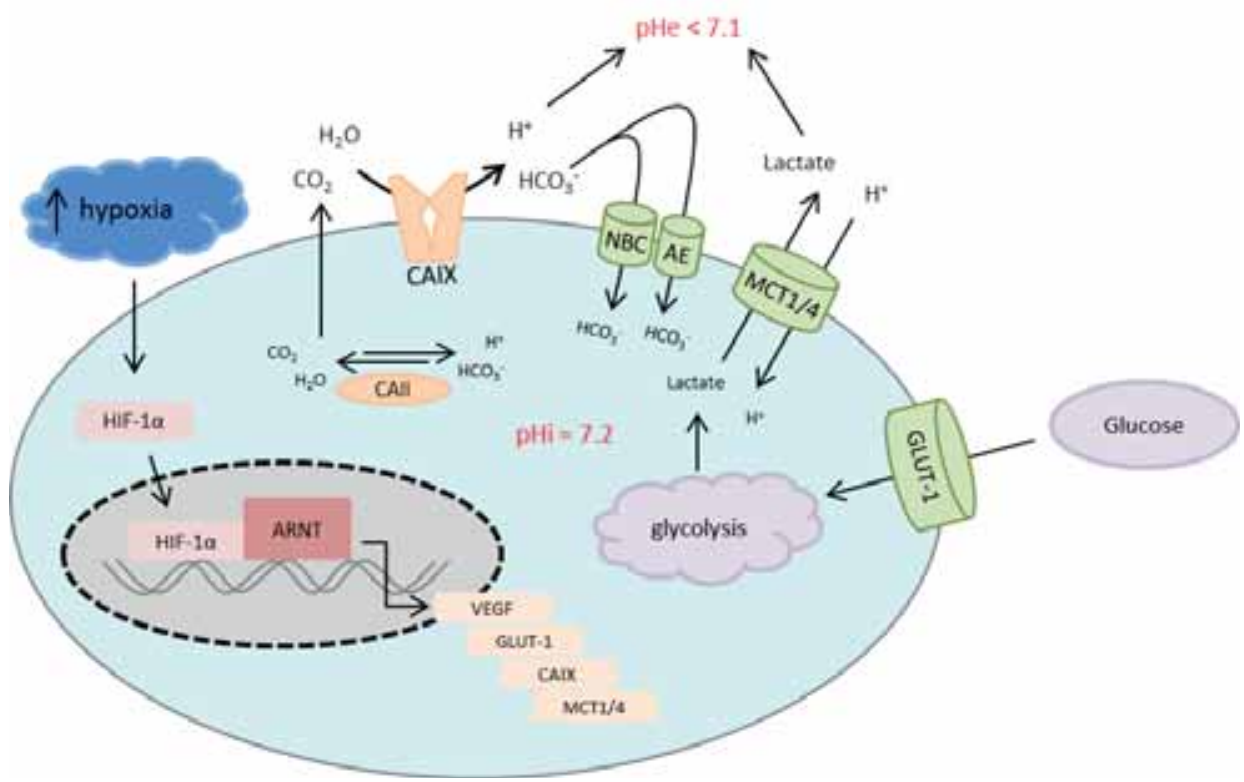

Figure 1.1 Schematic representation of the HIF-1 pathway with the effects on CAIX. HIF-1 $\alpha$ (Hypoxia Inducible Factor $1 \alpha$ ), ARNT (aryl hydrocarbon receptor nuclear translocator), CAll (Carbonic Anhydrase II), CAIX (Carbonic Anhydrase IX), NBC (sodium-bicarbonate co-transporter), AE (Anion Exchanger), MCT1/4 (Monocarboxylate transporters 1/4), GLUT-1 (glucose transporter-1), VEGF (vascular endothelial growth factor). Adapted from McDonald et al.(14). 
Sulfonamides are the most extensively studied group of small molecules. Fluorescent labeling of a sulfonamide showed to be promising for the detection of CAIX in a preclinical model since accumulation of the sulfonamide in the tumor was dependent on the oxygen status (22). However, fluorescent labeling is less applicable in the patient situation and therefore labeling of those compounds with radioactive tracers is under investigation.

\section{Targeting hypoxia to improve treatment outcome}

The efficacy of conventional anti-cancer therapies is impaired by the presence of tumor hypoxia. Therefore drugs have been developed specifically targeting those therapy resistant regions. In line with monitoring of hypoxia this targeting could be done by two approaches: direct targeting of the hypoxic cells or via inhibition of the hypoxia response proteins. Furthermore, the tumor hypoxic fraction could be modified by exposing subjects to high oxygen concentrations. This last approach is not investigated in this thesis, although modification of the oxygen concentrations is used to investigate the oxygen dependency of hypoxia PET tracers and a hypoxia targeting drug.

Direct targeting of hypoxic cells can be done using drugs that specifically get activated upon hypoxic conditions. These so called hypoxia-activated prodrugs are nontoxic under normal oxygen concentrations, only when oxygen is scarce the drug gets converted and becomes a cytotoxin that is able to crosslink DNA and induce cell damage. Tirapazamine is a well-known first generation hypoxia-activated cytotoxin and has a demonstrated anti-cancer activity. The efficacy of tirapazamine in combination with conventional radiotherapy and chemotherapy has been demonstrated in clinical phase II studies, but normal tissue toxicities seemed to be dose limiting (7). Clinical phase III studies however, did not show any beneficial effect (23). These negative results might partly be explained by the absence of patient selection on the presence of tumor hypoxia. Second generation hypoxia-selective cytotoxins were designed that were more stable and able to leave the cell after conversion. By the possibility of the active drug to diffuse, a so called 'bystander effect' was generated, which does not limit the toxic effect to the hypoxic cells of the tumor. However, the oxygen threshold for activation of these drugs is very critical. Since cytotoxicity in normal possibly slightly hypoxic tissues needs to be avoided, activation should only occur in severe hypoxic regions. As a consequence of the bystander effect the adjacent 'conventional therapy resistant' cells will then also be eliminated $(7,24)$. TH-302 is very promising new 
generation drug and has shown preclinical and clinical potential in the combination with several chemotherapeutics (25-27).

The other approach of sensitizing tumors to conventional therapies is by targeting the hypoxia response. As described above, CAIX is a good candidate for targeting because it plays a major role in the maintenance of the $\mathrm{pH}$ balance, which is favorable for the survival of cancer cells. The acidic extracellular $\mathrm{pH}$ promotes tumor cell migration, invasiveness, adaptation to micro environmental stresses and resistance to therapy (21). Inhibition of the enzymatic function of this protein would counteract the extracellular acidification and the intracellular alkalization, exacerbating the circumstances for the cancer cell and making it more susceptible for cell death. In line with CAIX as imaging target, both monoclonal antibodies and small molecular inhibitors can be used. First clinical trials for this approach are ongoing (22).

\section{Objective and outline of the thesis}

The main aim of this study is to integrate non-invasive tumor hypoxia imaging in the anticancer therapy for selecting subjects suitable for additional anti-hypoxia treatment. With this theranostic approach, which combines imaging and therapy, preclinical studies were conducted in line with the concept of personalized medicine (figure 1.2). The first objective of this thesis was to noninvasively visualize the hypoxic tumor environment to gain knowledge about the characteristics of the different tracers. This was done by either direct visualization of the hypoxic cells or by using the surrogate hypoxia marker CAIX. For the direct imaging of hypoxia clinically available and applicable PET tracers were used, while for the surrogate hypoxia imaging the specificity of a new CAIX tracer was assessed for the first time in this thesis. The second objective was to target the hypoxic tumor microenvironment, guided by hypoxia imaging where possible, with the aim to find an additional therapy that can overcome the pitfall of hypoxia-induced radiotherapy resistance. First clinical trials on the combination of TH-302 and chemotherapy show very promising results. The combination of this bioreductive drug with radiotherapy was investigated here for the first time in a preclinical setting with the aim to provide evidence for a clinical trial. For targeting of the hypoxia response protein CAIX, several drugs have been developed and described. In this thesis two of those CAIX specific small molecule inhibitors were tested in preclinical setting in combination with radiotherapy to investigate their potential in anticancer therapy. 


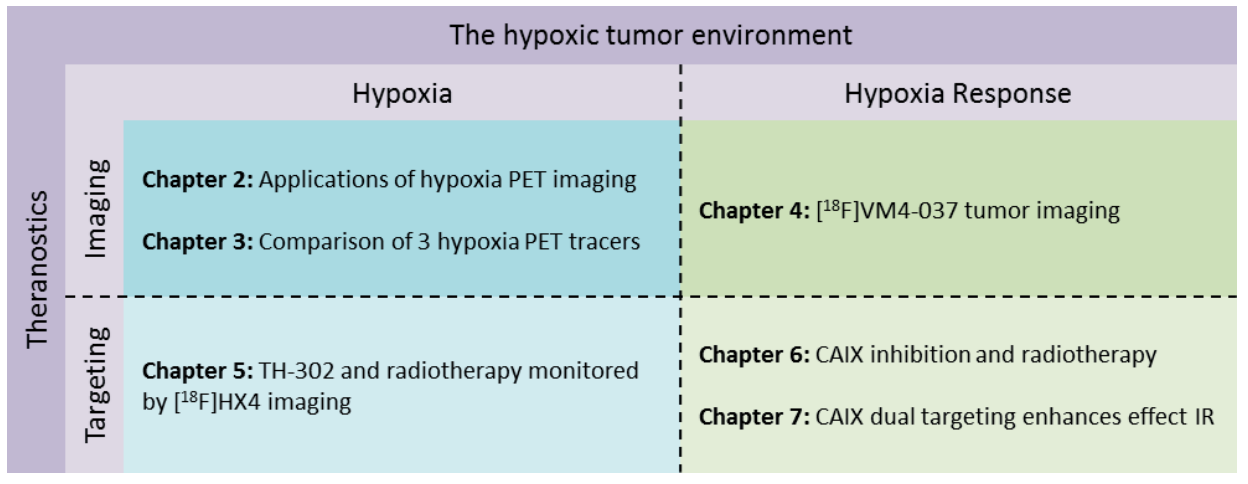

Figure 1.2: Schematic presentation of the thesis outline.

\section{Part I: Noninvasive imaging of the hypoxic tumor microenvironment}

The first part of the thesis focusses on the noninvasive imaging of the hypoxic tumor microenvironment. A comprehensive, objective overview of the recent literature is given in chapter 2, describing literature studying 2-nitroimidazole based hypoxia PET tracers in both pre-clinical and clinical setting. In chapter 3 the most promising, clinically used hypoxia PET tracers, $\left[{ }^{18} \mathrm{~F}\right] \mathrm{FMISO},\left[{ }^{18} \mathrm{~F}\right] \mathrm{FAZA}$ and $\left[{ }^{18} \mathrm{~F}\right] \mathrm{HX} 4$, were characterized with regard to optimal imaging time, tumor-to-blood ratios, spatial reproducibility and sensitivity to oxygen modification. This is the first study assessing these three hypoxia PET tracers in one preclinical tumor model, providing data for a fair comparison and a first indication on how the tracers relate to each other. Besides direct detection of the hypoxic regions, proteins expressed in response to hypoxia can be used for detection. Chapter 4 studied a new radioactive labeled PET tracer, $\left[{ }^{18} \mathrm{~F}\right] \mathrm{VM} 4-037$, directed against CAIX using microPET and biodistribution in two preclinical CAIX expressing tumor models.

\section{Part II: Targeting of the hypoxic tumor regions and the hypoxia response.}

The second part of this thesis studies drugs targeting hypoxia or the hypoxia response protein CAIX in combination with radiotherapy in order to improve treatment outcome. Chapter 5 studied the combination effect of TH-302 and radiotherapy in two preclinical tumor models. Modification of the tumor oxygen status was used to investigate the oxygen dependent efficacy of TH-302 in tumors. Furthermore, the hypoxic fraction before and after treatment was monitored by $\left[{ }^{18} \mathrm{~F}\right] \mathrm{HX} 4$ hypoxia PET imaging to study the effect of $\mathrm{TH}-302$ on the hypoxic fraction and to assess the correlation between pretreatment hypoxic fraction and treatment outcome. In chapter 6 CAIX was targeted as part of the hypoxia response using an indanesulfonamide with 
high specific affinity for CAIX. In chapter 7 a new approach was studied using a dual targeting drug that consists of a sulfamide directed against CAIX combined with a hypoxia targeting nitroimidazole moiety. In both chapters 6 and 7 the combination effect of CAIX targeting and radiotherapy was investigated.

Chapter 8 includes a general discussion on the monitoring and targeting of the hypoxic tumor environment in relation to the latest literature. Important applications and future directions will be discussed. 


\section{References}

1. Ferlay J, Steliarova-Foucher E, Lortet-Tieulent J, Rosso S, Coebergh JW, Comber H, et al. Cancer incidence and mortality patterns in Europe: estimates for 40 countries in 2012. Eur J Cancer. 2013;49:1374-403.

2. Vogelstein B, Papadopoulos N, Velculescu VE, Zhou S, Diaz LA, Jr., Kinzler KW. Cancer genome landscapes. Science. 2013;339:1546-58.

3. Hanahan D, Weinberg RA. Hallmarks of cancer: the next generation. Cell. 2011;144:646-74.

4. DeSantis CE, Lin CC, Mariotto AB, Siegel RL, Stein KD, Kramer JL, et al. Cancer treatment and survivorship statistics, 2014. CA Cancer J Clin. 2014;64:252-71.

5. Hockel M, Schlenger K, Aral B, Mitze M, Schaffer U, Vaupel P. Association between tumor hypoxia and malignant progression in advanced cancer of the uterine cervix. Cancer Res. 1996;56:4509-15.

6. Teicher BA. Hypoxia and drug resistance. Cancer Metastasis Rev. 1994;13:139-68.

7. Brown JM, Wilson WR. Exploiting tumour hypoxia in cancer treatment. Nat Rev Cancer. 2004;4:437-47.

8. Koritzinsky M, Wouters BG. The roles of reactive oxygen species and autophagy in mediating the tolerance of tumor cells to cycling hypoxia. Semin Radiat Oncol. 2013;23:252-61.

9. Dewhirst MW, Cao Y, Moeller B. Cycling hypoxia and free radicals regulate angiogenesis and radiotherapy response. Nat Rev Cancer. 2008;8:425-37.

10. Wouters BG, Koritzinsky M. Hypoxia signalling through mTOR and the unfolded protein response in cancer. Nat Rev Cancer. 2008;8:851-64.

11. Harris AL. Hypoxia--a key regulatory factor in tumour growth. Nat Rev Cancer. 2002;2:38-47.

12. Webb BA, Chimenti M, Jacobson MP, Barber DL. Dysregulated pH: a perfect storm for cancer progression. Nat Rev Cancer. 2011;11:671-7.

13. Swietach $\mathrm{P}$, Vaughan-Jones RD, Harris AL, Hulikova A. The chemistry, physiology and pathology of $\mathrm{pH}$ in cancer. Philos Trans R Soc Lond B Biol Sci. 2014;369:20130099.

14. McDonald PC, Winum JY, Supuran CT, Dedhar S. Recent developments in targeting carbonic anhydrase IX for cancer therapeutics. Oncotarget. 2012;3:84-97.

15. Lee ST, Scott AM. Hypoxia positron emission tomography imaging with 18f-fluoromisonidazole. Semin Nucl Med. 2007;37:451-61.

16. Padhani AR, Krohn KA, Lewis JS, Alber M. Imaging oxygenation of human tumours. Eur Radiol. 2007;17:861-72.

17. Dubois LJ, Lieuwes NG, Janssen MH, Peeters WJ, Windhorst AD, Walsh JC, et al. Preclinical evaluation and validation of [18F]HX4, a promising hypoxia marker for PET imaging. Proc Natl Acad Sci U S A. 2011;108:14620-5.

18. Halmos GB, Bruine de Bruin L, Langendijk JA, van der Laan BF, Pruim J, Steenbakkers RJ. Head and neck tumor hypoxia imaging by 18F-fluoroazomycin-arabinoside (18F-FAZA)-PET: a review. Clin Nucl Med. 2014;39:44-8.

19. Lopci E, Grassi I, Chiti A, Nanni C, Cicoria G, Toschi L, et al. PET radiopharmaceuticals for imaging of tumor hypoxia: a review of the evidence. Am J Nucl Med Mol Imaging. 2014;4:365-84.

20. Generali D, Berruti A, Brizzi MP, Campo L, Bonardi S, Wigfield S, et al. Hypoxia-inducible factor-1alpha expression predicts a poor response to primary chemoendocrine therapy and disease-free survival in primary human breast cancer. Clin Cancer Res. 2006;12:4562-8.

21. Pastorek J, Pastorekova S. Hypoxia-induced carbonic anhydrase IX as a target for cancer therapy: From biology to clinical use. Semin Cancer Biol. 2014.

22. Tafreshi NK, Lloyd MC, Bui MM, Gillies RJ, Morse DL. Carbonic anhydrase IX as an imaging and therapeutic target for tumors and metastases. Subcell Biochem. 2014;75:221-54. 
23. Reddy SB, Williamson SK. Tirapazamine: a novel agent targeting hypoxic tumor cells. Expert Opin Investig Drugs. 2009;18:77-87.

24. Wilson WR, Hay MP. Targeting hypoxia in cancer therapy. Nat Rev Cancer. 2011;11:393-410.

25. Chawla SP, Cranmer LD, Van Tine BA, Reed DR, Okuno SH, Butrynski JE, et al. Phase II study of the safety and antitumor activity of the hypoxia-activated prodrug $\mathrm{TH}-302$ in combination with doxorubicin in patients with advanced soft tissue sarcoma. J Clin Oncol. 2014;32:3299-306.

26. Liu Q, Sun JD, Wang J, Ahluwalia D, Baker AF, Cranmer LD, et al. TH-302, a hypoxia-activated prodrug with broad in vivo preclinical combination therapy efficacy: optimization of dosing regimens and schedules. Cancer Chemother Pharmacol. 2012;69:1487-98.

27. Sun JD, Liu Q, Wang J, Ahluwalia D, Ferraro D, Wang Y, et al. Selective tumor hypoxia targeting by hypoxia-activated prodrug TH-302 inhibits tumor growth in preclinical models of cancer. Clin Cancer Res. 2012;18:758-70. 



\section{Chapter 2}

\section{Current pre-clinical and clinical applications of hypoxia PET imaging using 2-nitroimidazoles}

Published in: Q J Nucl Med Mol Imaging. 2015;59:39-57

Sarah G.J.A. Peeters*, Catharina M.L. Zegers*, Ala Yaromina, Wouter van Elmpt, Ludwig Dubois, Philippe Lambin

*contributed equally 


\section{Abstract}

Hypoxia is a common characteristic of solid tumors and is associated with poor prognosis. Positron Emission Tomography (PET) can visualize tumor hypoxia in a noninvasive, 3-dimensional manner and can be used to acquire information longitudinally. Multiple 2-nitroimidazole based PET tracers are developed, validated and quantified in the search for the ideal hypoxia tracer and several tracers have shown to reliably represent tumor hypoxia. Furthermore, multiple studies describe the prognostic value of hypoxia PET imaging and the ability to monitor hypoxia during treatment. These applications can be of great potential and their role in treatment planning and modification needs to be further assessed with respect to personalized chemoradiation therapy. In this review we focus on the tracers that were positively validated in preclinical and clinical studies and report accurate quantification and visualization of hypoxia. The characteristics of these tracers are summarized for both preclinical and clinical studies. Furthermore, the clinical applications of hypoxia PET imaging are addressed with a focus on the ability to reliably monitor tumor hypoxia during treatment and the prognostic potential. Also the feasibility studies for hypoxia guided intensity modulated radiation therapy and the patient stratification for hypoxia targeted drugs are assessed. 


\section{Introduction}

Hypoxia in solid tumors is a negative prognostic factor for treatment outcome (1). Due to impaired and chaotic blood vessel development, cells outgrow the blood supply or vessels collapse leading to regions of chronic and acute hypoxia. Gold standard techniques for the detection of hypoxic regions consist of measuring oxygen concentrations directly with the Eppendorf oxygen sensitive electrode or immunohistochemistry of tumor biopsies. However both techniques are invasive and do not represent the tumor in three dimensions. In contrast, hypoxia position emission tomography (PET) imaging gives the opportunity to visualize the extent of hypoxia in a noninvasive manner, in three dimensions and additionally is able to provide information longitudinally, i.e. hypoxia status can be monitored over the course of treatment. For these reasons, hypoxia PET imaging has been widely studied over the last decade and is developing from a diagnostic technique that can detect hypoxia, into a tool that can be integrated versatile, i.e. in the treatment monitoring, outcome prediction and treatment targeting. Multiple tracers have been developed, validated and shown to exhibit different characteristics. In this review we focus on nitroimidazole-based tracers that were validated in preclinical and clinical studies and report accurate and reproducible quantification of hypoxia for multiple cancer sites. We discuss the ability of these hypoxia PET tracers to monitor treatment response and address their prognostic and predictive value for treatment outcome. Furthermore we assess current and future opportunities of hypoxia PET imaging as a tool to support treatment decisions (figure 2.1).

\section{Hypoxia PET Tracers}

Most PET tracers suitable for the detection of hypoxia are based on the principle of 2nitroimidazoles. After injection this molecule can diffuse freely from the vascular compartment to surrounding tissues where an electron reduction occurs which is reversed again under normal oxygen conditions. However, upon low oxygen conditions or in the presence of certain nitroreductase enzymes the molecule undergoes a second electron reduction and binds covalently to cellular components, causing the tracer to get trapped. Because of this specific accumulation in hypoxic tumor regions, and washout of the tracer in normal oxygenated tissues, these tumor areas can be clearly visualized by detection of the radioactive label connected to the tracer. The perfect hypoxia tracer should fulfill certain criteria, i.e. a large difference in washout rate between background and oxygen deprived tissues for high signal to noise ratios and 
specific and irreversible binding to hypoxic regions. Furthermore, the hydrophilicity of a tracer is of importance for the amount of clearance in well-oxygenated tissues while more lipophilic tracers accumulate more easily in hypoxic tissues. The first developed hypoxia PET tracer based on the 2-nitroimidazole principle is $\left[{ }^{18} \mathrm{~F}\right]$ labeled fluoromisonidazole $\left(\left[{ }^{18} \mathrm{~F}\right] \mathrm{FMISO}\right)$. However, some concerns were raised about the stability of $\left[{ }^{18} \mathrm{~F}\right] \mathrm{FMISO}$, the metabolite formation (2) and the slow clearance from background tissues, causing modest signal to noise ratios (3). Therefore, second generation hypoxia PET tracers have been developed to overcome these concerns. $\left[{ }^{18} \mathrm{~F}\right]$ Fluoroazomycin arabinoside $\left(\left[{ }^{18} \mathrm{~F}\right] \mathrm{FAZA}\right)$ is a nitroimidazole coupled to an arabinose sugar making the tracer more hydrophilic compared to $\left[{ }^{18} \mathrm{~F}\right] \mathrm{FMISO}$. Due to this property the visualization of the tracer should result in faster clearance from nonhypoxic/normal tissue and therefore have a better signal to noise ratio. [ ${ }^{18} \mathrm{~F}$ Fluoroerythronitroimidazole ( $\left.\left[{ }^{18} \mathrm{~F}\right] \mathrm{FETNIM}\right)$ is also a hydrophilic compound, causing higher tumor to background ratios compared to $\left[{ }^{18} \mathrm{~F}\right] \mathrm{FMISO}$ (4) $\left[{ }^{18} \mathrm{~F}\right] \mathrm{FETNIM}$ metabolite analysis showed high amounts of unchanged tracer present in plasma and urine of rats and dogs, whereas in the liver almost no intact $\left[{ }^{18}\right.$ F]FETNIM was present. Only low amounts of $\left[{ }^{18}\right.$ F]FETNIM binding to plasma proteins was observed (4). Tracers with a more lipophilic character were also of potential interest, leading to the development of the fluorinated etanidazole compounds $\left[{ }^{18} \mathrm{~F}\right] \mathrm{EF} 5$ and $\left[{ }^{18} \mathrm{~F}\right] \mathrm{EF} 3$. EF5 was first used as a monoclonal antibody for invasive detection of hypoxia in tissue biopsies (5). In the first study describing the radioactive labeling of this 2-nitroimidazole, analysis of metabolites showed that the tracer was unmodified (6). The chemical structure of $\left[{ }^{18} \mathrm{~F}\right] \mathrm{EF} 3$, and $\left[{ }^{18} \mathrm{~F}\right] \mathrm{EF5}$ are analogous to each other although small differences in chemical properties cause differences in biodistribution. Another tracer that tries to overcome the limitations of tumor to background ratio and aims for preferred pharmacokinetics and clearance properties is flortanidazole $\left[{ }^{18} \mathrm{~F}\right] \mathrm{HX} 4$ (7). A biodistribution study of $\left[{ }^{18} \mathrm{~F}\right] \mathrm{HX} 4$ showed high uptake in the bladder, liver and kidneys with the bladder wall being the most critical organ absorbing most radiation (8). The same study shows that $82 \%$ and $84 \%$ of the $\left[{ }^{18} \mathrm{~F}\right] \mathrm{HX} 4$ is intact and unmetabolized after 2 hours p.i. in the plasma and urine, respectively. Although many more hypoxia tracers have been developed and reviewed (9) this overview focuses on the ones that have been validated successfully in preclinical and clinical studies (figure 2.1). 


\section{Hypoxia PET tracers}

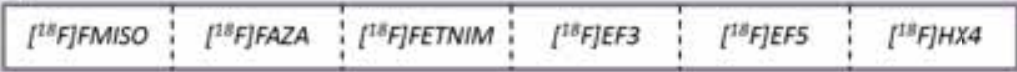

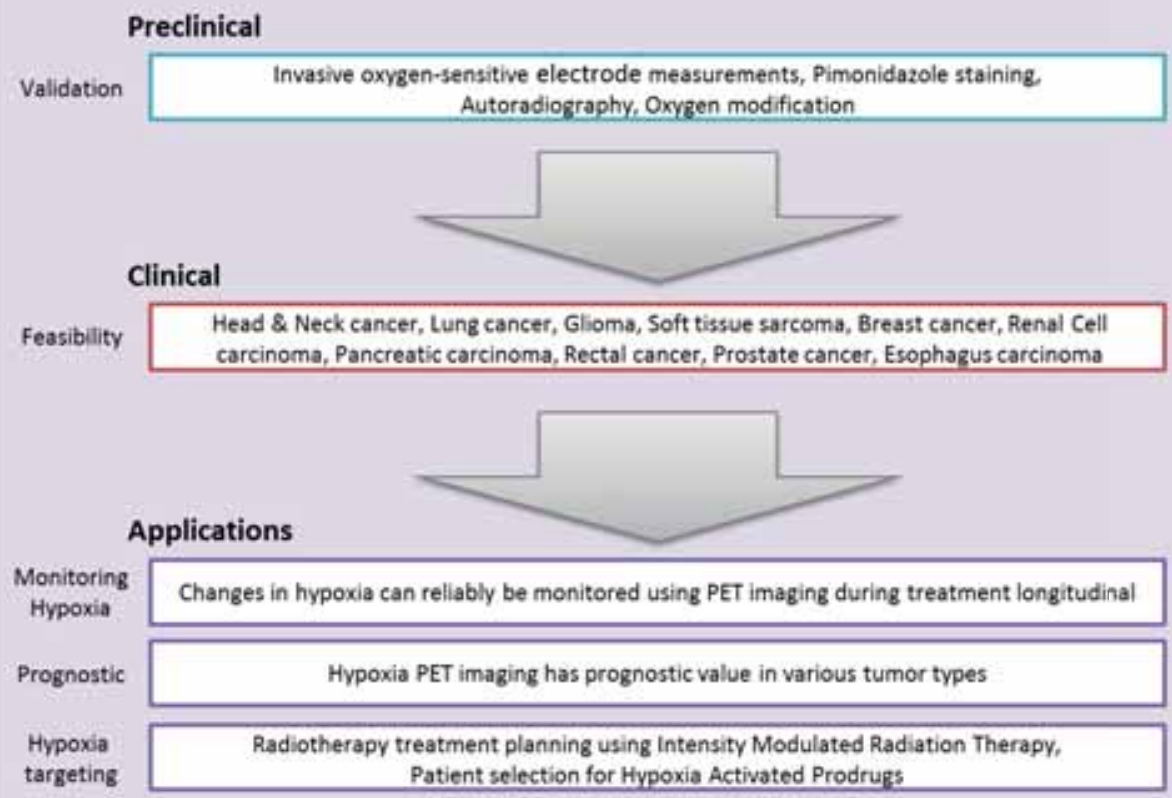

Figure 2.1 Overview of the outline of the review

\section{Validation}

An important step in the assessment of a successful hypoxia PET tracer is the validation of these agents, to ensure that the observed PET uptake in the tumor represents actual tumor hypoxia. First of all this can be done in a direct comparison between the hypoxia PET characteristics, like standardized uptake value or tumor to background ratio, and the gold standard which assesses the partial oxygen pressure in the tumor, i.e. Eppendorf $\mathrm{pO}_{2}$ measurements. In addition, hypoxia PET imaging can be correlated to immunohistochemical markers which directly or indirectly represent tumor hypoxia. Another validation method to detect hypoxia specific tracer accumulation is by exposing subjects to modified oxygen concentration breathing just before and after the tracer injection. Preclinical studies form the ideal platform for the first feasibility 
and validation studies. However, since pharmacokinetics differ between humans and rodents, further validation in clinical trials is necessary.

\section{$\left[{ }^{18} \mathrm{~F}\right] \mathrm{FMISO}$}

The validation of $\left[{ }^{18} \mathrm{~F}\right] \mathrm{FMISO}$ has been performed in multiple studies which assessed the uptake and distribution of $\left[{ }^{18} \mathrm{~F}\right] \mathrm{FMISO}$ in different tumor models using a range of validation techniques. A selection of these studies is reported here.

The first assessment of oxygen dependency of $\left[{ }^{18} \mathrm{~F}\right] \mathrm{FMISO}$ was demonstrated in porcine livers (10). Feasibility of hypoxia imaging using $\left[{ }^{18} \mathrm{~F}\right] \mathrm{FMISO}$ was shown in tumor-bearing mice although no correlation was found with oxygen partial pressure as detected by polarographic oxygen-sensitive electrodes. However, autoradiography showed more $\left[{ }^{18}\right.$ F]FMISO uptake in the tumor of ambient air breathing animals compared to better oxygenated tumors in animals exposed to carbogen $\left(95 \% \mathrm{O}_{2}, 5 \%\right.$ $\mathrm{CO}_{2}$ ) breathing (11). In a rat rhabdomyosarcoma tumor model a heterogeneous pattern was observed on autoradiography for both $\left[{ }^{18} \mathrm{~F}\right] \mathrm{FMISO}$ uptake and pimonidazole staining (12). Furthermore a significant correlation was observed between hypoxic volumes assessed by $\left[{ }^{18} \mathrm{~F}\right] \mathrm{FMISO}$ PET and pimonidazole immunostaining in tumor sections. A weak but significant correlation was found for 10 different human primary head and neck xenograft tumor models comparing $\left[{ }^{18} \mathrm{~F}\right] \mathrm{FMISO}$ uptake with pimonidazole staining, however, the correlation was stronger when the pattern of hypoxia was ribbon-like and had a micro-regional distribution instead of a homogeneous distribution (13). In addition a relationship between $\left[{ }^{18} \mathrm{~F}\right] \mathrm{FMISO}$ and pimonidazole was observed in Dunning R3327-AT bearing rats, although some mismatch was found possibly due to incomplete washout of $\left[{ }^{18} \mathrm{~F}\right] \mathrm{FMISO}$ from the wellperfused areas (14). Moreover, $\left[{ }^{18} \mathrm{~F}\right]$ FMISO PET imaging was able to detect hypoxic micro-metastases as confirmed by autoradiography and pimonidazole staining (15). Responsiveness of $\left[{ }^{18} \mathrm{~F}\right] \mathrm{FMISO}$ to different oxygen concentrations $\left(10 \% \mathrm{O}_{2}\right.$, normal air or carbogen) was shown in a SCCVII tumor model and corresponded with pimonidazole uptake (16).

Dogs bearing spontaneous sarcomas were imaged using $\left[{ }^{18} \mathrm{~F}\right] \mathrm{FMISO}$ and these data confirmed the Eppendorf $\mathrm{pO}_{2}$ measurements (17). Similar findings were observed in R3327-AT bearing rats where oxygen probe measurements broadly corresponded with $\left[{ }^{18}\right.$ F]FMISO uptake (18). However, Chang et al. reported that although the overall data were positive, large variation was observed between individual data pairs indicating that $\left[{ }^{18}\right.$ F]FMISO PET imaging might be problematic (19). Using the combined diffusion- 
retention dynamic information of two $\left[{ }^{18} \mathrm{~F}\right] \mathrm{FMISO}$ scans performed at 0-15 min p.i. and $4 \mathrm{~h}$ p.i., reported to give an accurate measure of the median oxygen concentration comparable to average oxygen probe measurements (20).

In clinical setting, most validation studies using the Eppendorf $\mathrm{pO}_{2}$ measurements are performed in the head and neck cancer patient population, because of the relative easy accessible tumor location. Several publications correlate the $\left[{ }^{18} \mathrm{~F}\right] \mathrm{FMISO}$ PET uptake with pO2 measurements, however with contradictory results. Mortensen et al. (21) observed no correlation, while Gagel et al. (22) and Zimny et al. (23) reported respectively a moderate and strong correlation. The validation of hypoxia PET imaging in 13 patients with accessible soft tissue sarcomas was performed by Bentzen et al. in combination with Eppendorf pO2 measurements (24). A lack of concordance between the PET results and the oxygen measurements was observed, suggesting that $\left[{ }^{18} \mathrm{~F}\right] \mathrm{FMISO}$ PET imaging was not able to detect tumor hypoxia or its extend as defined by $\mathrm{pO} 2$ measurements.

The clinical $\left[{ }^{18}\right.$ F]FMISO characteristics were also compared to the immunohistochemical staining of endogenous hypoxia markers. A weak but significant correlation between the $\left[{ }^{18} \mathrm{~F}\right] \mathrm{FMISO}$ hypoxic volume and HIF1 $\alpha$ expression was found in head and neck cancer patients $(25,26)$. In patients with non-small-cell-lung cancer (NSCLC) no correlation was observed between the $\left[{ }^{18} \mathrm{~F}\right] \mathrm{FMISO}$ uptake and the immunohistochemical tumor markers microvessel density, HIF1 $\alpha$, VEGF and GLUT1 (27). In contrast, in patients with glioma brain tumors a significant correlation between $\left[{ }^{18}\right.$ F]FMISO PET imaging and the vascular endothelial growth factor receptor (VEGF) and the cell proliferation associated antigen (Ki67) expression was observed. The relationship between tumor hypoxia and Ki67 expression could be explained by the fact that both tumor parameters were related to the tumor grade (28). In addition HIF1 $\alpha$ expression showed a (non-significant) trend towards increased uptake. In breast cancer patients there was no correlation between hypoxia PET imaging using $\left[{ }^{18} \mathrm{~F}\right] \mathrm{FMISO}$ and HIF1- $\alpha$ expression, however, a significant correlation was observed with the proliferation marker Ki67 (29). In renal cell carcinoma an increased microvessel density was present in comparison to the normal kidney parenchyma, however, this was not correlated to $\left[{ }^{18}\right.$ F]FMISO PET imaging (30).

\section{$\left[{ }^{18} \mathrm{~F}\right] \mathrm{FAZA}$}

Feasibility of $\left[{ }^{18} \mathrm{~F}\right]$ FAZA visualization was assessed in a squamous cell carcinoma of the head and neck ( $\mathrm{FaDu})$, a cervix ( $\mathrm{SiHa}$ ) tumor model and two murine tumor models. 
$\left[{ }^{18}\right.$ F]FAZA uptake based on microPET imaging was compared to Eppendorf $\mathrm{pO}_{2}$ measurements, autoradiography and pimonidazole immunohistochemistry staining. Based on the invasive Eppendorf electrode measurements all tumors were assigned as being very hypoxic which was confirmed by pimonidazole staining and $\left[{ }^{18} \mathrm{~F}\right] \mathrm{FAZA}$ PET images. Furthermore significant correlations were observed between the autoradiography images and pimonidazole uptake, while overall an inverse relationship was found with the perfusion marker Hoechst 33342 (31, 32). These data were confirmed in the same murine tumor model on multiple time points post tracer injection and supplemented with biodistribution data confirming the previous findings (33). In addition [ $\left.{ }^{18} \mathrm{~F}\right]$ FAZA uptake was shown to correlate with the mRNA expression of CA9 and GLUT-1 after fragmenting the tumor in milligram-sized portions, which, according to the authors, allows multiple pairwise measurements at the smallest volumetric scale possible (34). Autoradiography of $\left[{ }^{18} \mathrm{~F}\right] \mathrm{FAZA}$ distribution in a SQ20b xenograft model also showed a clear positive relationship with pimonidazole and CAIX staining (35). Another validation study for $\left[{ }^{18} \mathrm{~F}\right] \mathrm{FAZA}$ uptake was performed by exposing rhabdomyosarcoma bearing rats to carbogen breathing which resulted in a decreased uptake of the hypoxia tracer (36). Moreover, $\left[{ }^{18} \mathrm{~F}\right]$ FAZA PET correlated with the hypoxic fraction as measured by oxyLite $\mathrm{pO}_{2}$, Electron Paramagnetic Resonance (EPR) and ${ }^{19} \mathrm{~F}-\mathrm{MRI}$ suggesting that $\left[{ }^{18} \mathrm{~F}\right] \mathrm{FAZA}$ PET is a promising imaging technique for hypoxia evaluation (36) In an orthotropic brain tumor model the detection of hypoxia using $\left[{ }^{18} \mathrm{~F}\right]$ FAZA PET imaging revealed low tumor to background ratios at 18-20 days post tumor implantation while high ratios were observed after 28-30 days (37). Bioluminescence imaging of the luciferase activity induced under the control of 'hypoxia responsive elements' confirmed that hypoxia development started around day 18 and increased from this day onward.

\section{$\left[{ }^{18}\right.$ F]FETNIM}

In preclinical setting, the paper describing the synthesis and first validation of $\left[{ }^{18}\right.$ F]FETNIM reports high tumor to blood (TBR) and tumor to muscle (TMR) at $4 \mathrm{~h}$ p.i. as detected by biodistribution. In addition on autoradiography hypoxic regions could be differentiated from necrotic regions (38). In NSCLC patients, Hu et al. observed a significant correlation between $\left[{ }^{18}\right.$ F]FETNIM hypoxia PET imaging and the immunohistochemical markers HIF1 $\alpha$, GLUT-1 and VEGF. Since all these immunohistochemical markers are up-regulated in the absence of oxygen, this result provides the indirect evidence that $\left[{ }^{18}\right.$ F]FETNIM uptake gives an accurate indication of tumor hypoxia (39). 


\section{$\left[{ }^{18} \mathrm{~F}\right]$ EF5 \& $\left[{ }^{18} \mathrm{~F}\right]$ EF3}

Studies comparing $\left[{ }^{18} \mathrm{~F}\right] \mathrm{EF} 5$ with $\mathrm{pO}_{2}$ or pimonidazole assessed hypoxic fractions are not available. However, validation of $\left[{ }^{18} \mathrm{~F}\right] \mathrm{EF} 5$ in a HCT116 model reported feasibility of PET acquisition based on ex vivo gamma counting (40). Feasibility of $\left[{ }^{18} \mathrm{~F}\right] \mathrm{EF} 5$ imaging was also shown in a rat model where tumors with high hypoxic values demonstrated a TMR between 1.2-1.7 while for low hypoxic tumors a TMR below unity was observed (41). In the tumor models H460, HCT116 and PC3, PET imaging of $\left[{ }^{18} \mathrm{~F}\right] \mathrm{EF5}$ was performed followed by autoradiography and staining for immunofluorescence hypoxia marker EF5. The spatial relationship was found to be dependent on the tumor model being the highest in the PC3 model followed by the HCT116 and the H460 model. Notably the uptake of $\left[{ }^{18} \mathrm{~F}\right] \mathrm{EF} 5$ was influenced by the simultaneous injection of nonradioactive EF5 which might have important clinical implication when hypoxia tracer is combined with hypoxia targeting drugs with similar properties (42). Furthermore, Silén et al. reported in preclinical head and neck xenograft models that high $\left[{ }^{18}\right.$ F]EF5 PET uptake was observed in tumor models having high CAIX, GLUT1 and HIF1 $\alpha$ expression, while low values of these endogenous markers were detected in models with low $\left[{ }^{18} \mathrm{~F}\right] \mathrm{EF5}$ uptake (43).

A first validation study for $\left[{ }^{18} \mathrm{~F}\right] \mathrm{EF} 3$ revealed a biodistribution based TBR ranging from 1.2 to 2.9 and ranking of the different tumor models based on $\left[{ }^{18} \mathrm{~F}\right] \mathrm{EF} 3$ uptake was comparable with ranking based on the amount of hypoxia as detected by EF5 immunofluorescence (44). Furthermore a significant correlation was observed between $\left[{ }^{18} \mathrm{~F}\right] \mathrm{EF} 3$ and EF5 immunofluorescence after exposing the tumor bearing animals to either carbogen or $10 \%$ oxygen breathing (45) while pharmacological intervention could not improve TMR (46).

\section{$\left[{ }^{[8} \mathrm{F}\right] \mathrm{HX} 4$}

The first validation of $\left[{ }^{18} \mathrm{~F}\right] \mathrm{HX} 4$ was performed in a rat rhabdomyosarcoma model and demonstrated a significant spatial relationship at tumor-regional level between $\left[{ }^{18} \mathrm{~F}\right] \mathrm{HX} 4$ distribution and pimonidazole staining indicating that $\left[{ }^{18} \mathrm{~F}\right] \mathrm{HX} 4$ does accumulate specifically in hypoxic regions (47). Furthermore $\left[{ }^{18} \mathrm{~F}\right] \mathrm{HX} 4$ uptake was causally related to the tumor oxygenation, since exposing animals to altered oxygen concentrations resulted in a dependent $\left[{ }^{18} \mathrm{~F}\right] \mathrm{HX} 4$ accumulation in the tumor. Carlin et al. reported that the tracer distribution on autoradiography showed a clear positive relationship with pimonidazole and CAIX staining, indicating that HX4 accumulates in the hypoxic regions (35). 
All PET tracers discussed here show feasibility of detecting hypoxic tumor regions although each tracer has its own characteristics. Since studies are performed in different animal tumor models, using various conditions and validation methods, comparisons between different tracers is difficult. For some tracers this is assessed in separate quantification studies (vide infra).

Overall, from the clinical validation studies we can conclude that there is limited evidence linking hypoxia PET imaging with actual oxygen pressure measurements. This might be due to the limitation of oxygen electrode measurements or biopsies to quantify the heterogeneous presence of hypoxia in 3D, which is assessed with hypoxia PET, or the limitation of hypoxia PET to visualize the presence of tumor hypoxia on a micrometer scale given the spatial resolution of current PET CT imaging. Although the uptake of several hypoxia PET tracers correlated with pimonidazole staining in preclinical studies, the use of exogenous pimonidazole hypoxia marker is not very common in clinical studies. Therefore endogenous markers are used which report diverse and inconsistent correlations within or between different tumor types. This diversity might at least partially be explained by possible oxygen-independent regulation of these proteins. Although the often used endogenous hypoxia markers HIF1 $\alpha$, CAIX and GLUT1 are regulated in response to low oxygen concentrations, their expression does not always correlate with the hypoxic regions (48). Therefore, the results of comparisons between hypoxia tracers and endogenous markers should be interpreted with caution.

\section{Quantification}

Quantification of the tracers' uptake is most often determined on the combined $\mathrm{PET} / \mathrm{CT}$ images, providing both functional (hypoxia) and anatomical information. The amount of tracer present in the tumor can be quantified as the "standardized uptake value" (SUV) or tumor-to-background ratio. Based on predefined thresholds, the hypoxic volume or hypoxic fraction can be calculated. Reproducibility is another important measure in hypoxia PET imaging. Quantification of the hypoxic fraction has no use if data are not reproducible over a short or even longer period. Therefore both quantification and reproducibility are evaluated.

Comparative studies in preclinical tumor models can easily quantify the uptake of multiple tracers within one tumor model hereby providing important information on 
the performance of the tracers with regard to each other (figure 2.2). Furthermore the implementation of experimental procedures to increase or decrease the hypoxic fraction can easily be applied to investigate the tracer's behavior to these altered conditions.

A first comparison study investigating both $\left[{ }^{18} \mathrm{~F}\right.$ FMISO with $\left[{ }^{18} \mathrm{~F}\right] \mathrm{FAZA}$ PET uptake in the Walker 256 rat carcinosarcoma model showed an increase in SUV and TBR values from 1 to 3 hours p.i. The $\left[{ }^{18}\right.$ F]FMISO uptake values were higher than those for $\left[{ }^{18} \mathrm{~F}\right]$ FAZA (49). Contradicting results were obtained in EMT6, AR42J and A431 tumor models where biodistribution based TMR was significantly higher for $\left[{ }^{18} \mathrm{~F}\right] \mathrm{FAZA}$ as compared with $\left[{ }^{18} \mathrm{~F}\right] \mathrm{FMISO}(50)$. The presence of hypoxia was confirmed using invasive electrode $\mathrm{pO}_{2}$ measurements. Furthermore, exposing animals to $100 \% \mathrm{O}_{2}$ breathing conditions revealed reduced $\left[{ }^{18}\right.$ F]FAZA PET uptake compared to ambient air breathing conditions, which was confirmed by autoradiography. These results were confirmed in another study on A431 tumor bearing mice (51). Although the specific uptake in the tumor was higher for $\left[{ }^{18} \mathrm{~F}\right] \mathrm{FMISO}$, the TBR and TMR were significantly higher for $\left[{ }^{18} \mathrm{~F}\right] \mathrm{FAZA}$ on both PET imaging and biodistribution. Sensitivity to increased oxygen concentrations was reported for $\left[{ }^{18} \mathrm{~F}\right] \mathrm{FAZA}$ uptake detected by PET and biodistribution. Accumulation of $\left[{ }^{18} \mathrm{~F}\right] \mathrm{FAZA}$ was indicated to be crucial in the first hour post injection (52). Exposing tumor bearing animals to enhanced oxygen concentrations either from 1 hour pre injection till 3 hours post injection or only from 2 minutes pre injection till 1 hour post injection gave similar modified results compared to ambient air breathing.

A preclinical comparative study on $\left[{ }^{18}\right.$ F]FETNIM and $\left[{ }^{18} \mathrm{~F}\right] \mathrm{FMISO}$ in $\mathrm{C} 3 \mathrm{H}$ mammary carcinoma bearing mice used biodistribution to investigate TBR and TMR under ambient air and carbogen breathing. For both tracers, TBR was decreased under carbogen breathing with higher ratios for $\left[{ }^{18} \mathrm{~F}\right]$ FETNIM $(5.8 \pm 2.5$ for air and $2.8 \pm 1.3$ for carbogen) than for $\left[{ }^{18}\right.$ F]FMISO (4.3 \pm 2.0 for air and $2.3 \pm 0.5$ for carbogen) whereas TMR was comparable between the two tracers $\left(\left[{ }^{18} \mathrm{~F}\right]\right.$ FETNIM $6.2 \pm 2.1$ and $\left[{ }^{18} \mathrm{~F}\right] \mathrm{FMISO}$ $6.4 \pm 3.3$ both in normal air) (53).

A comparative study investigated the TBR of $\left[{ }^{18} \mathrm{~F}\right] \mathrm{EF} 3$ and $\left[{ }^{18} \mathrm{~F}\right] \mathrm{FMISO}$ in the same rat rhabdomyosarcoma model. At $2 \mathrm{~h}$ p.i. TBR of $\left[{ }^{18} \mathrm{~F}\right] \mathrm{FMISO}$ was significantly higher than $\left[{ }^{18} \mathrm{~F}\right] \mathrm{EF} 3$, but $\left[{ }^{18} \mathrm{~F}\right] \mathrm{EF} 3$ values at $4 \mathrm{~h}$ p.i were comparable to those of $\left[{ }^{18} \mathrm{~F}\right] \mathrm{FMISO}$. This demonstrates that with respect to TBR values $\left[{ }^{18} \mathrm{~F}\right] \mathrm{EF} 3$ is not superior to $\left[{ }^{18} \mathrm{~F}\right] \mathrm{FMISO}$ (54). This was partially confirmed in a FSA II tumor model where $\left[{ }^{18} \mathrm{~F}\right] \mathrm{FMISO}$ TMR was significantly higher only at 55 and 330 minutes compared to $\left[{ }^{18} \mathrm{~F}\right] \mathrm{EF} 3$ and not at other 
time points. Furthermore, $\left[{ }^{18} \mathrm{~F}\right] \mathrm{EF} 3 \mathrm{TMR}$ remained low under carbogen breathing, while $\left[{ }^{18} \mathrm{~F}\right] \mathrm{FMISO}$ values kept increasing, suggesting that $\left[{ }^{18} \mathrm{~F}\right] \mathrm{EF} 3$ is more sensitive to reduced hypoxia by carbogen breathing (55). Overall, $\left[{ }^{18} \mathrm{~F}\right] \mathrm{EF} 3$ seems to be a good hypoxia tracer although it might not be superior to $\left[{ }^{18} \mathrm{~F}\right] \mathrm{FMISO}$.

A comparative study performed in our laboratory characterized $\left[{ }^{18} \mathrm{~F}\right] \mathrm{FMISO},\left[{ }^{18} \mathrm{~F}\right] \mathrm{FAZA}$ and $\left[{ }^{18} \mathrm{~F}\right] \mathrm{HX} 4$ within one rhabdomyosarcoma tumor model on TBR, reproducibility and reversibility (56). Within this tumor model $\left[{ }^{18} \mathrm{~F}\right] \mathrm{HX} 4$ reached the highest TBR at $3 \mathrm{~h}$ p.i. while $\left[{ }^{18} \mathrm{~F}\right] \mathrm{FAZA}$ reached a stable TBR at $2 \mathrm{~h}$ p.i. and $\left[{ }^{18} \mathrm{~F}\right] \mathrm{FMISO}$ did not reach a plateau. Two PET acquisitions over a 48 hour time period revealed high reproducibility and good overlap in hypoxic regions for both $\left[{ }^{18} \mathrm{~F}\right] \mathrm{FMISO}$ and $\left[{ }^{18} \mathrm{~F}\right] \mathrm{HX} 4$, while $\left[{ }^{18} \mathrm{~F}\right] \mathrm{FAZA}$ did perform worse on this characteristic. Furthermore, $\left[{ }^{18} \mathrm{~F}\right] \mathrm{FMISO}$ showed to be sensitive to decreasing hypoxic fractions while $\left[{ }^{18} \mathrm{~F}\right] \mathrm{FAZA}$ and $\left[{ }^{18} \mathrm{~F}\right] \mathrm{HX} 4$ were responsive to increasing hypoxia. These alterations of oxygen concentrations were introduced by exposing animals to either high or low oxygen concentrations 2.5 hours after tracer injection while PET acquisition was performed just before and immediately after this modification (56). In a SQ20b head and neck xenograft model microPET imaging was performed at only one time point, 80-90 minutes p.i., and demonstrated a tumor to muscle ratio of $1.6 \pm 0.4$ for $\left[{ }^{18} \mathrm{~F}\right] \mathrm{FMISO}, 1.4 \pm 0.1$ for $\left[{ }^{18} \mathrm{~F}\right] \mathrm{HX} 4$ and $1.3 \pm 0.3$ for $\left[{ }^{18} \mathrm{~F}\right] \mathrm{FAZA}$ (35).

The previously described tracers $\left[{ }^{18} \mathrm{~F}\right] \mathrm{FMISO},\left[{ }^{18} \mathrm{~F}\right] \mathrm{FAZA},\left[{ }^{18} \mathrm{~F}\right] \mathrm{HX} 4,\left[{ }^{18} \mathrm{~F}\right] \mathrm{EF}$, and $\left[{ }^{18} \mathrm{~F}\right]$ FETNIM were used for hypoxia PET imaging in patients with head and neck cancer (figure 2.2; $\left[{ }^{18} \mathrm{~F}\right] \mathrm{HX} 4$ image of head and neck cancer patient). A huge variation of data is reported with no standardized imaging acquisition protocol or image analysis. The PET imaging time point p.i. ranges from $1.5 \mathrm{~h}$ to $4 \mathrm{~h}$ p.i. In addition, the selected background tissue ('normal' tissue, muscle, blood, cerebellum) and the thresholds to define tumor hypoxia are diverse; for example, selecting the voxels with a tumor-to-background $\geq$ $1.2, \geq 1.25, \geq 1.3, \geq 1.4, \geq 1.5$, or selecting voxels above the background uptake +3 standard deviations. This makes it very difficult or even impossible to compare the different tracers based on the currently available clinical literature. Only one study compared two tracers in the same patient population; however the authors used different acquisition protocols (57). $\left[{ }^{18} \mathrm{~F}\right] \mathrm{FMISO}$ imaging was performed at $2 \mathrm{~h}$ p.i., while $\left[{ }^{18} \mathrm{~F}\right] \mathrm{HX} 4$ imaging was performed at $1.5 \mathrm{~h}$ p.i. The study showed similar hot spots for both tracers and comparable TBR ratios for the time points used. 


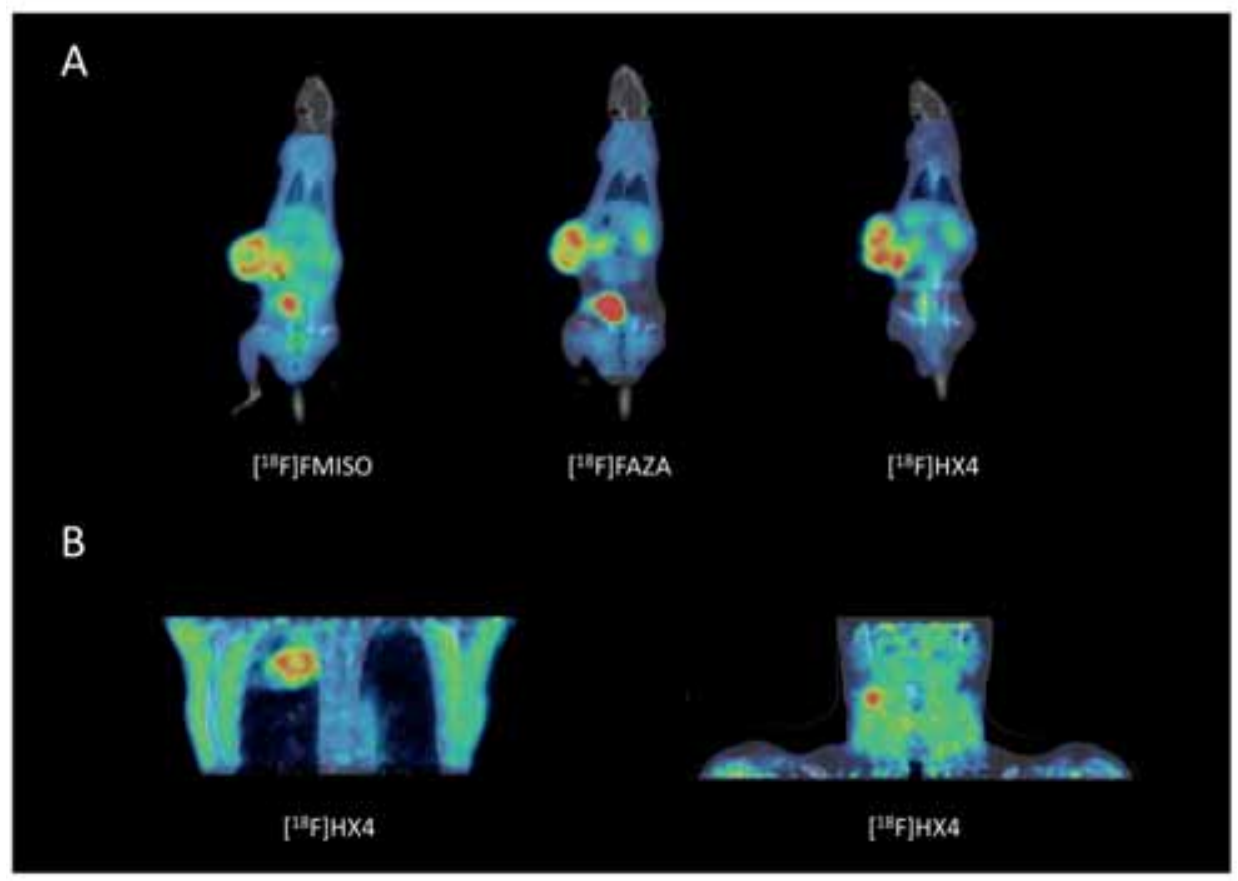

Figure 2.2 A) Preclinical PET/CT images representing [18F]FMISO, [18F]FAZA and [18F]HX4 uptake in a rhabdomyosarcoma bearing rat at 4 hours post injection. B) The clinical images visualize a lung (left) and a Head and Neck (right) cancer patient at 4 hour

In patients with NSCLC the tracers $\left[{ }^{18} \mathrm{~F}\right] \mathrm{FMISO},\left[{ }^{18} \mathrm{~F}\right] \mathrm{FAZA}$ and $\left[{ }^{18} \mathrm{~F}\right] \mathrm{HX} 4$ were used to visualize tumor hypoxia (2; $\left[{ }^{18} \mathrm{~F}\right] \mathrm{HX} 4$ image of lung cancer patient). Rasey et al. already quantified tumor hypoxia, using $\left[{ }^{18} \mathrm{~F}\right] \mathrm{FMISO}$, in 1996 . They observed tumor hypoxia in all NSCLC lesions, however a broad range of the extent of hypoxia was shown with a fractional hypoxic volume ranging from $1.3-94.7 \%$ (2). $\left[{ }^{18}\right.$ F $]$ FAZA PET/CT imaging in patients with NSCLC was performed by Postema et al. (58) and Trinkaus et al. (59) who observed tumor hypoxia in respectively $54 \%$ and $65 \%$ of the patients. These results were confirmed with $\left[{ }^{18} \mathrm{~F}\right] \mathrm{HX} 4$, reporting an increased uptake in $72 \%$ of all NSCLC lesions (60). All these results show that the majority of NSCLC lesions are hypoxic and therefore lung cancer patients could be good candidates for hypoxia targeted therapies.

$\left[{ }^{18} \mathrm{~F}\right] \mathrm{FMISO}$ was also used to quantify tumor hypoxia in patients with brain cancer, pancreatic cancer and renal cell carcinoma. In 11 patients with glioblastoma an average tumor-to-blood ratio of $1.5 \pm 0.2$ was reported (61). This $\left[{ }^{18} \mathrm{~F}\right] \mathrm{FMISO}$ TBR was significantly correlated to the biological aggressiveness measured by tumor growth 
kinetics on MRI. In addition it was observed that the $\left[{ }^{18} \mathrm{~F}\right] \mathrm{FMISO}$ uptake (tumor to cerebellum ratio) was significantly higher for the patients with glioblastoma $(2.7 \pm 0.6)$, in comparison to the other glioma patients $(1.2 \pm 0.1)(62)$. These results were confirmed by Yamamoto et al., who observed no hypoxia (TBR $<1.2)$ in the grade I and II gliomas, and a significantly increasing TBR for grade III (1.8 \pm 0.6$)$ and IV (2.5 \pm 0.4$)$ (63). Therefore, in this patient population, the quantification of tumor hypoxia could contribute to the staging of patients with glioma.

In patients with pancreatic carcinoma, a visual increased uptake of $\left[{ }^{18} \mathrm{~F}\right] \mathrm{FMISO}$ was only observed in 2 out of 10 patients, however all patients showed a TBR $\geq 1.4$, indicating uptake in hypoxic regions. Based on the difficulty to visually assess tumor hypoxia in patients with pancreatic cancer, hypoxia PET imaging should be combined with other modalities for lesion localization (64). The $\left[{ }^{18} \mathrm{~F}\right] \mathrm{FMISO}$ uptake in 11 patients with renal cell carcinoma was mildly increased, with an average tumor SUVmax of 1.3 \pm 0.3 in comparison to the normal kidney tissue $1.1 \pm 0.2$ (30).

In patients with rectal cancer both $\left[{ }^{18} \mathrm{~F}\right] \mathrm{FMISO}$ and $\left[{ }^{18} \mathrm{~F}\right] \mathrm{FAZA}$ was used to assess tumor hypoxia. Loi et al. performed $\left[{ }^{18} \mathrm{~F}\right.$ ]FMISO PET imaging, as part of a phase 1 trial in 6 patients with rectal cancer. Tumor hypoxia was observed in $67 \%$ of the primary lesions and in $83 \%$ of the metastases at baseline (65). In addition it was reported that $\left[{ }^{18} \mathrm{~F}\right]$ FAZA uptake in rectal tumors was significantly higher than the reference tissues, with an average tumor to muscle ratio of 2.0 (66). An additional, important message in this manuscript is a practical issue regarding the influence of scattered activity from the bladder, which can influence the quantitative analysis of $\left[{ }^{18} \mathrm{~F}\right] \mathrm{FAZA}$ PET uptake in the rectum. $\left[{ }^{18} \mathrm{~F}\right] F A Z A$ was additionally used to investigate tumor hypoxia in patients with prostate carcinoma, with negative results. The attempt to visualize tumor hypoxia in 14 patients with histology proven prostate cancer failed to show any significant uptake in the tumor lesions (67). [ ${ }^{18}$ F]FETNIM hypoxia PET imaging was used to describe tumor hypoxia in 28 patients with esophageal carcinoma. Increased uptake of $\left[{ }^{18} \mathrm{~F}\right]$ FETNIM inside the primary tumor was observed which was the first evidence for hypoxia PET quantification in esophageal cancer.

From all these studies, it can be concluded that the imaging contrast of nitroimidazole based hypoxia tracers is limited, however sufficient to quantify tumor hypoxia and extract valuable information for most clinical tumor types. 


\section{Pharmacokinetic analysis}

Besides static PET imaging, quantification of tumor hypoxia can be performed using dynamic PET acquisitions and image analysis. This pharmacokinetic analysis can be used to provide additional tumor specific values of tracer retention and tumor perfusion. In a preclinical investigation in Dunning R3327-AT prostate tumor bearing nude rats the relationship between the $\left[{ }^{18} \mathrm{~F}\right] \mathrm{FMISO}$ pharmacokinetic rate constants was assessed, using an irreversible two-tissue compartment model, in relationship to pO2 measurements. They showed that the rate-constant k3, representing the trapping rate, was better than the standard tumor-to-plasma ratio, to identify tumor hypoxia (68). In addition Busk et al. performed pharmacokinetic modeling of $\left[{ }^{18} \mathrm{~F}\right] \mathrm{FAZA}$ in three murine squamous cell carcinomas using also an irreversible two-tissue compartment model. The time activity curves of the tumors varied between the different tumor types. Correlation of the rate-constants to static $3 \mathrm{~h}$ p.i. $\left[{ }^{18} \mathrm{~F}\right] \mathrm{FAZA}$ PET imaging, showed a poor correlation for $\mathrm{k} 3$ for 2 out of 3 tumor types, however a good correlation was observed with $\mathrm{Ki}$, the influx rate constant; $(k 1 \times k 3) /(k 2+k 3)$ (69). Clinically pharmacokinetic analysis of $\left[{ }^{18} \mathrm{~F}\right] \mathrm{FMISO}$ was only performed in patients with head and neck cancer. In agreement with pre-clinical data, an irreversible 2-tissue compartment model was used to describe the kinetics of $\left[{ }^{18} \mathrm{~F}\right] \mathrm{FMISO}(70,71)$. A positive correlation was observed between the influx rate $\mathrm{Ki}$ and the standard tumor to blood ratio, measured on static $\left[{ }^{18} \mathrm{~F}\right]$ FMISO images (70). Another study used pharmacokinetic modeling to differentiate between tumor perfusion and hypoxia, providing the opportunity to stratify patients in three categories, (i) high perfusion, no hypoxia, (ii) perfusion and hypoxia and (iii) severe hypoxia and reduced perfusion, which could be related to the radiotherapy outcome. For $\left[{ }^{18} \mathrm{~F}\right] \mathrm{FAZA}$, kinetic analysis was clinically performed in patients with NSCLC. Three different compartment models were evaluated; the reversible single-tissue, reversible two-tissue and irreversible two-tissue compartment model. In this patient population, the reversible two-tissue compartment model is found to best represent $\left[{ }^{18} \mathrm{~F}\right] \mathrm{FAZA}$ kinetics (72). Using this model, the calculated volume of distribution $\left(\mathrm{V}_{\mathrm{T}} \mathrm{k} 1 / \mathrm{k} 2(1+\mathrm{k} 3 / \mathrm{k} 4)\right)$ showed a good correlation to the statically measured SUV values and TBR. From the clinical literature we observe a difference in model preference for $\left[{ }^{18} \mathrm{~F}\right] \mathrm{FMISO}$, irreversible and $\left[{ }^{18} \mathrm{~F}\right] \mathrm{FAZA}$, reversible, which could be explained by the different properties of the tracers. However, studies were performed in different patient populations (head and neck versus NSCLC). To make a useful comparison kinetic analysis of the different tracers within the same patient population is necessary. Note that for both $\left[{ }^{18} \mathrm{~F}\right] \mathrm{FMISO}$ and $\left[{ }^{18} \mathrm{~F}\right] \mathrm{FAZA}$, the kinetic parameters $\mathrm{Ki}$ or $\mathrm{V}_{\mathrm{T}}$ were correlated to the static parameter TBR or SUV, which justifies a standard static analysis for these PET tracers. 


\section{Applications}

PET hypoxia imaging can be used for multiple applications. It has been shown to have a prognostic value and can be useful in the monitoring of tumor hypoxia during the course of treatment. Furthermore the assessment of tumor hypoxia can be used to select patients that might benefit from a certain therapy.

\section{Prognostic / predictive value of hypoxia PET imaging}

In a preclinical setting the prognostic value of $\left[{ }^{18} \mathrm{~F}\right] \mathrm{FAZA}$ was assessed by performing a hypoxia PET scan combined with the invasive oxygen sensitive electrode measurement, followed by a single dose of irradiation (55Gy). Most animals were exposed to ambient oxygen breathing while a fraction of the animals was exposed to carbogen breathing from 10 minutes prior to and during both hypoxia measurements and radiotherapy. Both methods could distinguish between the more and less hypoxic tumors resulting in significantly higher local tumor control (73). Another study reported that discrimination between normoxic and hypoxic tumors based on pretreatment $\left[{ }^{18} \mathrm{~F}\right]$ FAZA PET imaging could predict the effectiveness of the combined treatment of radiotherapy with the hypoxia specific cytotoxin tirapazamine (74).

In the clinic the relationship between hypoxia PET imaging and treatment outcome was investigated by several groups (Table 1 ). The use of $\left[{ }^{18} \mathrm{~F}\right] \mathrm{FMISO},\left[{ }^{18} \mathrm{~F}\right] \mathrm{FETNIM}$ and $\left[{ }^{18} \mathrm{~F}\right] \mathrm{EF} 5$ was correlated with the outcome of head and neck cancer patients. Lehtio et al. showed in 21 patients that the fractional hypoxic volume, measured before treatment on $\left[{ }^{18}\right.$ F]FETNIM PET imaging, was predictive for 3-years overall survival (75). In addition it was reported that there is a significant decrease in disease free survival for hypoxic versus non-hypoxic tumors $(76,77)$. On the other hand, no correlation was shown between outcome and pre- or mid-treatment $\left[{ }^{18} \mathrm{~F}\right] \mathrm{FMISO}$ imaging for patients treated with several cycles of platinum based chemotherapy and IMRT (Intensity Modulated Radiation Therapy). However, this conclusion was based on a 3-year local and regional progression-free survival rate of $100 \%$ and $95 \%$. Only one patient showed regional failure after treatment, but did not have a positive $\left[{ }^{18} \mathrm{~F}\right] \mathrm{FMISO}$ PET/CT scan during treatment (78). The association between local progression-free survival and $\left[{ }^{18} \mathrm{~F}\right] \mathrm{FMISO}$ PET imaging before and during chemo-radiotherapy was investigated and showed that $\left[{ }^{18} \mathrm{~F}\right]$ FMISO PET imaging during treatment had a higher prognostic power than baseline hypoxia imaging (79). In addition, parameters extracted from the kinetic analysis of $\left[{ }^{18} \mathrm{~F}\right]$ FMISO PET imaging and its changes during treatment were highly correlated to radiotherapy outcome $(80,81)$. 
Rischin et al. for the first time demonstrated the predictive value of $\left[{ }^{18} \mathrm{~F}\right] \mathrm{FMISO}$ in patients with head and neck cancer randomized in a Phase II trial for chemoradiotherapy alone or in combination with hypoxic cell cytotoxin tirapazamine. Pretreatment $\left[{ }^{18}\right.$ F]FMISO tumor hypoxia was associated with a high risk of locoregional failure after chemoradiotherapy alone. A considerable improvement in locoregional control was found in patients with hypoxic tumors treated with tirapazamine, whereas tirapazamine could not improve the outcome of chemoradiotherapy in non-hypoxic tumors (82).

Several studies investigated the prognostic value of hypoxia PET imaging for patients with head and neck cancer in comparison to standard metabolic PET imaging using $\left[{ }^{18}\right.$ F]FDG. In an univariate analysis it was shown that the nodal stage and $\left[{ }^{18} \mathrm{~F}\right] \mathrm{FMISO}$ PET parameters (TBR and hypoxic volume) were significantly correlated with survival, while the $\left[{ }^{18} \mathrm{~F}\right]$ FDG SUV max $_{\text {was not }}$ (83). These results were confirmed by another study which observed a significant correlation between FMISO PET uptake and chemotherapy response, while $\left[{ }^{18}\right.$ F]FDG uptake was not correlated (26). A relationship between overall survival, the hypoxic subvolume, defined on $\left[{ }^{18} \mathrm{~F}\right] \mathrm{EF} 5 \mathrm{PET}$ imaging, and the metabolic active tumor volume, defined on the $\left[{ }^{18}\right.$ F]FDG-PET was observed (84). However, also in this study, the hypoxia PET uptake was stronger correlated with overall survival in comparison to $\left[{ }^{18} \mathrm{~F}\right] \mathrm{FDG}$. All these studies show the prognostic potential of hypoxia PET imaging over metabolic $\left[{ }^{18}\right.$ F]FDG PET imaging. However, Thorwarth et al. showed in a small cohort of patients $(\mathrm{N}=12)$ that a parameter based on the voxel-wise comparison of both tracers quantifying discrepancies between $\left[{ }^{18} \mathrm{~F}\right]$ FMISO and $\left[{ }^{18} \mathrm{~F}\right]$ FDG PET was the most significant variable (85), suggesting that a combination of two tracers on a voxel level may increase both their prognostic significance.

Hypoxia PET imaging was also used to predict the outcome of patients with lung cancer. It was observed that the TBR and hypoxic volume defined on $\left[{ }^{18} \mathrm{~F}\right] \mathrm{FETNIM} \mathrm{PET}$ were predictors of overall survival in a univariate analysis, while the $\left[{ }^{18} \mathrm{~F}\right] \mathrm{FDG}$ uptake was not significant (86). The $\left[{ }^{18} \mathrm{~F}\right] \mathrm{FMISO}$ PET time activity curve type (i.e. rapid washout, delayed washout or accumulation) and a tumor to muscle ratio of 2.0 were shown to be good parameters to predict tumor response (87). On the other hand no significant difference was observed in disease-free survival between NSCLC patients $(\mathrm{N}=17)$ having a hypoxic or a non-hypoxic lesions (59). For patients with glioma, $\left[{ }^{18}\right.$ F]FMISO PET imaging was observed to be prognostic for treatment outcome (28). The majority of patients who died of progressive disease had positive $\left[{ }^{18} \mathrm{~F}\right] \mathrm{FMISO}$ 
uptake, while for those that were still alive, $\left[{ }^{18} \mathrm{~F}\right] \mathrm{FMISO}$ was negative in most cases. In addition, an increased TBR or hypoxic volume defined on $\left[{ }^{18} \mathrm{~F}\right] \mathrm{FMISO}$ PET imaging was found to be significantly related to a decreased overall survival in patients with newly diagnosed glioma $(88,89)$.

$\left[{ }^{18}\right.$ F]FETNIM uptake was significantly correlated with progression-free survival in patients with cervical carcinoma (90). In addition, the $\left[{ }^{18}\right.$ F]FETNIM SUV ${ }_{\text {max }}$ was the best predictor of treatment response in comparison to tumor length, diameter, and $\mathrm{SUV}_{\text {mean }}$ in patients with esophageal carcinoma (91). Cheng et al. evaluated $\left[{ }^{18} \mathrm{~F}\right] \mathrm{FMISO}$ PET imaging in patients with breast cancer for the prediction of treatment resistance to hormonal therapy (29). They observed a significant correlation between baseline $\left[{ }^{18}\right.$ F]FMISO uptake and clinical outcome. Using a TBR threshold $\geq 1.2$ to quantify tumor hypoxia allowed correct prediction for $88 \%$ of the patients with progressive disease. Comparison of $\left[{ }^{18} \mathrm{~F}\right]$ FMISO PET imaging at baseline and follow-up showed that the patients with a partial response or stable disease showed a decreasing trend of the $\left[{ }^{18} \mathrm{~F}\right] \mathrm{FMISO}$ TBR, while patients with progressive disease did not. In patients with renal cell carcinoma, the presence of baseline tumor hypoxia was related to a shorter progression-free survival, however overall survival was not related to $\left[{ }^{18} \mathrm{~F}\right] \mathrm{FMISO}$ uptake. Also changes in tumor hypoxia during treatment were not prognostic for progression-free or overall survival (92).

In the previous paragraph "validation" we have described that there is limiting evidence linking hypoxia PET imaging with actual oxygen pressure measurements in patients. However given the prognostic value of hypoxia PET imaging in various tumor types, the question is how we should approach validation of our current hypoxia PET imaging techniques in clinical practice. Given the highly prognostic value of hypoxia PET imaging, which is in general stronger than the other available (imaging) parameters, it shows that there is added prognostic and maybe predictive value in hypoxia imaging. Therefore, hypoxia PET imaging could be integrated in clinical trials which have the aim to improve loco-regional control or survival, especially for hypoxia modifying treatments.

\section{Monitoring tumor hypoxia during treatment}

Experimental and clinical data demonstrate that tumor hypoxia plays an important role in malignant progression and resistance to anti-cancer treatments. Hypoxia PET imaging can be used as a noninvasive tool to evaluate the effectiveness of therapy to eliminate hypoxic tumor cells and adapt treatment accordingly. This was studied in 
some preclinical studies giving a first indication about the possibilities. However treatment schedules as they are applied to patients are hard to mimic in preclinical setting.

Acquisition of a $\left[{ }^{18}\right.$ F]FMISO PET scan was performed before and after the treatment of a HT29 xenograft tumor using the antivascular compound vadimezan (93). A reduced $\left[{ }^{18} \mathrm{~F}\right] \mathrm{FMISO}$ uptake 24 hours after vadimezan administration was observed only in a part of the treatment group. Quantification of the tumor microvasculature revealed that this was most likely due to changes in perfusion and could probably not be assigned to changes in hypoxia. The antiangiogenic treatment with sunitinib also reduced the $\left[{ }^{18}\right.$ F]FMISO uptake after 7 days of treatment in an orthotopic glioma model while the cerebral blood volume (CBV) was increased and the vessel permeability was decreased (94). Control animals were unaffected in CBV but had high hypoxic values. However, in a renal cell carcinoma xenograft model, the hypoxic fraction detected by $\left[{ }^{18} \mathrm{~F}\right] \mathrm{FMISO}$ was increased after 2, 3 and 7 days of treatment with Sorafenib, an antiangiogenic drug, compared to control (95). This was supported by immunohistochemical data indicating that the mean vessel density decreased, while necrosis in the tumor increased.

\section{Table 2.1 Overview of clinical studies associated with outcome.}

TBR = tumor to background ratio (independent of selected background), HV = hypoxic volume (independent of used threshold), $\mathrm{HF}=$ hypoxic fraction (independent of used threshold)

${ }^{1}$ Definitive radiotherapy ( $\left.R T ; N=4\right)$, preoperative $R T(N=13)$, postoperative $R T(N=1)$, interrupted $R T$ (44Gy; $\mathrm{N}=1), \mathrm{RT}$ in combination with chemotherapy $(\mathrm{N}=2)$ and patients without $\mathrm{RT}(\mathrm{N}=2)$

${ }^{2}$ No statistical test was performed, however none of the patients experienced local failure and only one patient experienced regional failure 5 months after treatment completion, this was not a patient with a positive FMISO PET.

${ }^{3} \mathrm{P}$-values are dependent on the threshold used to define hypoxic volume

${ }^{4} \mathrm{HRP}=$ ratio between tracer retention potential and perfusion ratio

${ }^{5}$ No statistical test was performed, however reported was that change of curve type was correlated with patient outcome

${ }^{6}$ Definitive radiotherapy with or without chemotherapy or definitive resection with or without adjuvant radiotherapy

${ }^{7}$ Multivariate analysis was performed on 50 patients (which had both FDG and FMISO imaging)

${ }^{8}$ Randomized trial, treatment of patients was radiotherapy in combination with either tirapazamine and cisplatine (CIS-TPZ), or cisplatin and fluorouracil (CIS-FU).

${ }^{9}$ Patients were treated with standard chemoradiation or a combination of carboplatin, paclitaxel, gefitinib and radiation.

${ }^{10}$ No statistical analysis was performed, however of the patients who died, 7 had positive FMISO uptake, 1 equivocal and 1 negative, while of those alive, 5 had negeative uptake and equivocal in 1.

11 Imaging was performed before operation or stereotactic biopsy $(\mathrm{N}=11)$, after resection and before radiation therapy $(\mathrm{N}-7)$ or after resection and radiotherapy $(\mathrm{N}=11)$. 


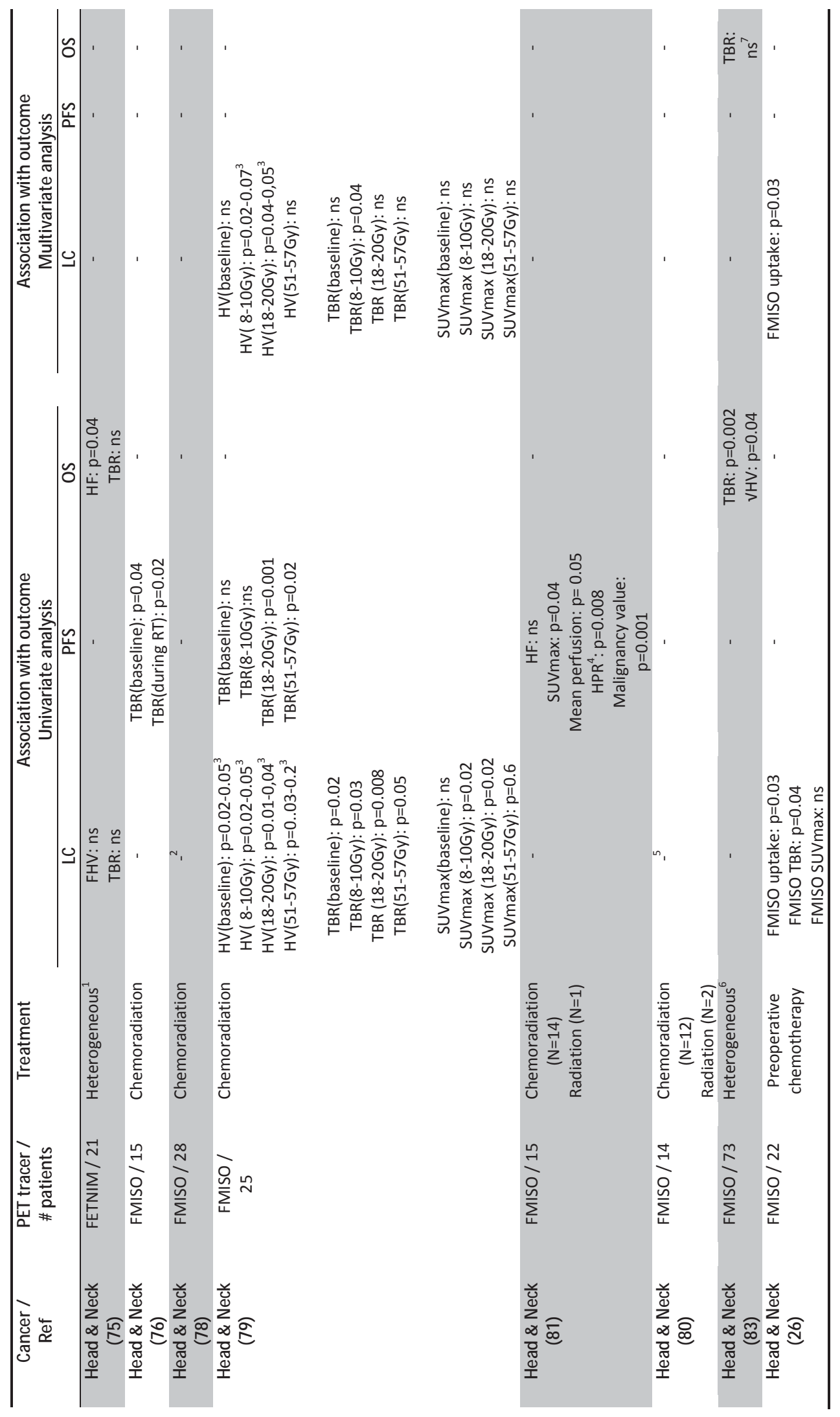




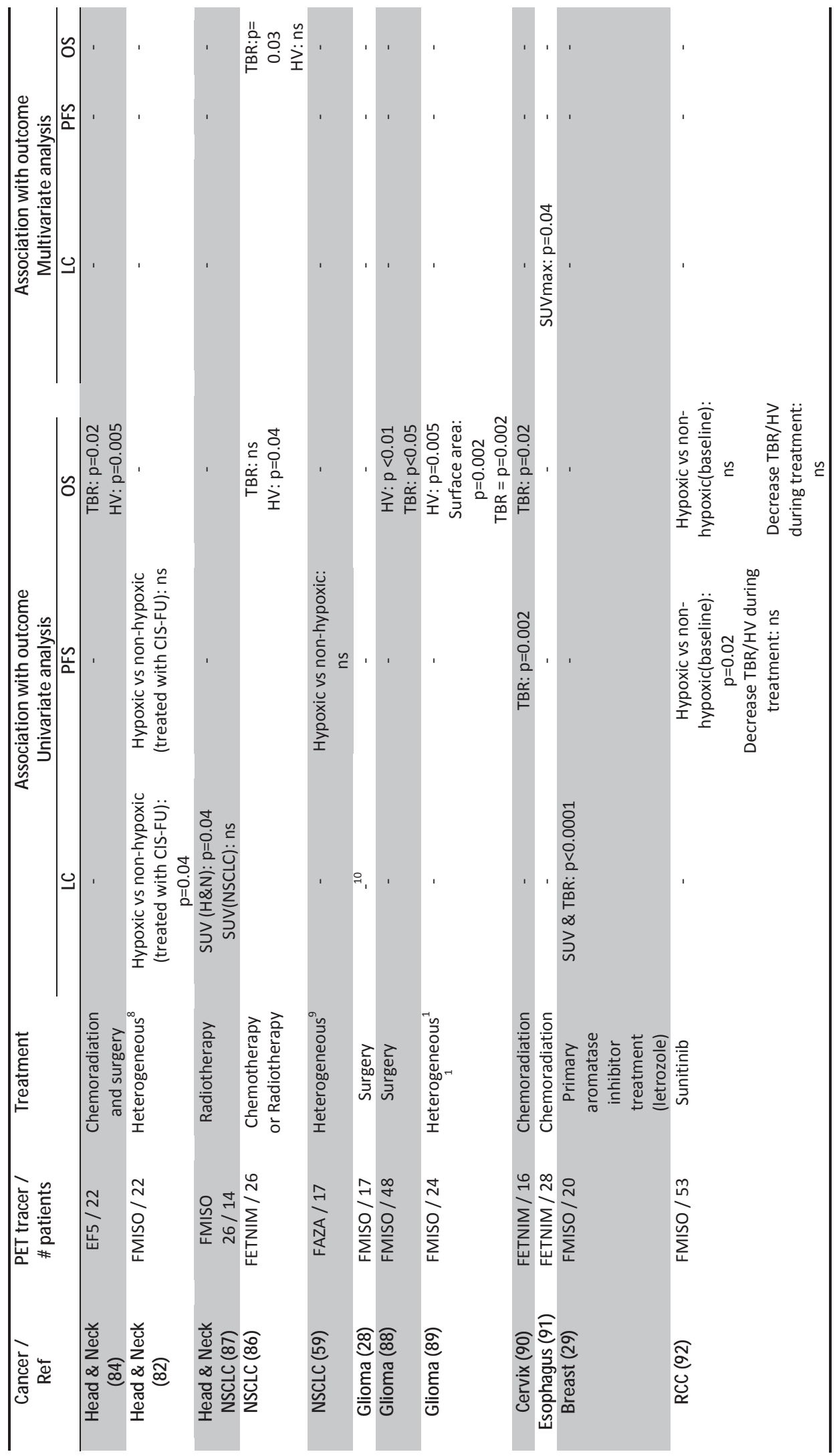


In a preclinical cervix tumor model two $\left[{ }^{18}\right.$ F]FAZA PET scans were acquired on consecutive days before the start of fractionated radiotherapy, followed by a $\left[{ }^{18} \mathrm{~F}\right] \mathrm{FAZA}$ PET scan after 4 and 10 fractions of 2.5Gy (96). Voxel to voxel analysis revealed a good reproducibility on the pretreatment scans, but the overall $\left[{ }^{18} \mathrm{~F}\right] \mathrm{FAZA}$ uptake changed over treatment. Treatment of A431 bearing animals with the pan-erbB tyrosine kinase inhibitor $\mathrm{Cl}-1033$ was monitored using hypoxia PET imaging pretreatment and on day 3,6 or 7 (97). Therapy was shown to be effective since shrinkage of the treated tumor occurred. This was reflected in the $\left[{ }^{18}\right.$ F]FAZA uptake which decreased over treatment from day 0 to day 3 and an even further decrease on day $6 .\left[{ }^{18} \mathrm{~F}\right]$ FMISO imaging at day 7 confirmed the low uptake in the $\mathrm{Cl}-1033$ treated animals while the control animals had high tracer uptake for both $\left[{ }^{18} \mathrm{~F}\right] \mathrm{FAZA}$ and $\left[{ }^{18} \mathrm{~F}\right] \mathrm{FMISO}$. The efficacy of another tyrosine kinase inhibitor (Gefitinib) was tested in the same tumor model showing similar results (98). The amount of hypoxia in the treatment group is reduced as measured by $\left[{ }^{18} \mathrm{~F}\right]$ FAZA PET imaging, while the control group remained hypoxic. $\left[{ }^{18} \mathrm{~F}\right] \mathrm{EF} 5$ PET imaging was used to monitor treatment response of a bioreductive drug combined with radiotherapy (99). This showed a reduction in SUV comparing $24 \mathrm{~h}$ post treatment with a pretreatment scan indicative for tumor response.

These preclinical studies show a discrepancy in hypoxia PET imaging after the use of antivascular treatments which is possibly caused by differences in perfusion as a result of the treatment. The monitoring of other anticancer therapies did show consistent results, indicating that hypoxia PET imaging is suitable for this kind of studies.

Several clinical studies focused on the monitoring of hypoxia using noninvasive PET imaging during treatment. In patients with head and neck squamous cell carcinoma a decreased uptake of $\left[{ }^{18} \mathrm{~F}\right] \mathrm{FMISO}$ after treatment with neoadjuvant chemotherapy was reported (100). In addition, in $89 \%$ of the patients $\left[{ }^{18} \mathrm{~F}\right] \mathrm{FMISO}$ uptake was completely absent after treatment with one cycle of chemotherapy (5-fluororacil \& carboplatin) and 40Gy of IMRT (78). Servagi-Vernat reported the uptake of $\left[{ }^{18} \mathrm{~F}\right] \mathrm{FAZA}$ before and during concomitant chemo-radiotherapy in the primary tumor and the involved lymph nodes. In all lesions, the fractional hypoxic volume and SUV $V_{\max }$ decreased after 17 fractions of radiotherapy (101). The changes in $\left[{ }^{18}\right.$ F]FMISO kinetics after treatment with (chemo-)radiotherapy were also studied. Three different curve types were defined, representing a severe, intermediate and low degree of hypoxia (80). Based on these curves, a decreased amount of hypoxia in the majority of patients was shown. From these studies we can conclude that in patients with head and neck cancer, 
hypoxia decreases during (chemo-) radiation treatment, indicating therapy induced reoxygenation.

In patients with NSCLC, the evaluation of hypoxia changes during treatment was investigated using $\left[{ }^{18} \mathrm{~F}\right] \mathrm{FMISO}$ PET imaging. One study observed an average decrease in tumor hypoxia during radiotherapy, however the changes were unpredictable and only one patient had no detectable tumor hypoxia by the end of radiotherapy (102). Another study observed no significant changes in $\left[{ }^{18} \mathrm{~F}\right] \mathrm{FMISO}$ uptake before and during treatment with chemo-radiotherapy (103). On the other hand in a study using $\left[{ }^{18} \mathrm{~F}\right]$ FAZA, tumor hypoxia was eliminated after treatment with chemo-radiotherapy in the majority of NSCLC patients (59). For cervical carcinoma $\left[{ }^{18}\right.$ F]FAZA PET imaging was performed before, during and after radiotherapy. Before the start of radiotherapy positive $\left[{ }^{18}\right.$ F]FAZA uptake was observed in 5 out of 15 patients. During treatment persistent hypoxia was observed in 4 patients, while after radiotherapy no remaining hypoxia was observed (104). As part of a multimodality imaging trial $\left[{ }^{18} \mathrm{~F}\right.$ FMISO imaging was performed in sarcomas before, during and after chemotherapy (105). All soft tissue sarcomas presented a hypoxic volume before the start of treatment, which remained present during and after treatment. The changes of tumor hypoxia during treatment were diverse among the patient population. Rajendran et al. confirms these results, reporting tumor hypoxia in 14 out of 19 patients with soft tissue sarcoma, which decreased, increased or remained stable during treatment in 6, 2 and 2 patients respectively (106). Both studies reported that there was no association between the hypoxic volume in soft tissue sarcomas and the tumor grade.

Comparing the results of the different tumor types, it is striking that in majority of head and neck studies a decrease of tumor hypoxia is observed during treatment, while these results are diverse in NSCLC, cervical cancer and sarcomas. This might suggest that tumor hypoxia is more persistent in these cancer types, or the given treatment is not effective in eradicating the hypoxic cells. This might also explain the results of Overgaard et al. showing that oxygen modification in addition to radiotherapy provides a significantly increased tumor control for head and neck cancer patients, however not for uterine cervix or NSCLC (107).

\section{Hypoxia targeting}

Based on clinical studies showing an association between hypoxia and treatment outcome, hypoxia PET imaging might be a powerful tool for patient selection and 
treatment adaptation. The available literature mainly focuses on the adaptation of radiotherapy based on hypoxia PET imaging information and so far only feasibility studies have been reported. Furthermore the patients that are sensitive to hypoxia targeted treatments could be selected, moving in the direction of individualized medicine.

One possibility to target hypoxic tumor subvolumes is to escalate radiation dose. The feasibility of $\left[{ }^{18} \mathrm{~F}\right] \mathrm{FMISO}$ PET based IMRT to the hypoxic tumor volume was investigated for 8 patients with head and neck cancer (108). They were able to perform a doseescalation (78Gy) to the hypoxic tumor volume in 6 out of 8 patients without increasing the normal tissue doses. Additionally, $\left[{ }^{18} \mathrm{~F}\right] \mathrm{FMISO}-$ based boost plans could provide an increased tumor control probability without increasing expected complications (109). A theoretical model also indicated the potential for dose sparing by focusing the dose to the radioresistant hypoxic tumor volumes (110). In addition, Chang et al. performed a biological modeling study in 8 HNSCC patients, using $\left[{ }^{18} \mathrm{~F}\right.$ FMISO PET imaging for radiotherapy dose painting (111). They compared the standard radiotherapy plan with uniform dose escalation and hypoxia-based dose painting. Hypoxia-based dose painting demonstrated the advantage to increase the tumor control probability without increasing the normal tissue toxicity, therefore increasing the chances for an uncomplicated tumor control probability. These results confirm that it is theoretically possible to perform hypoxia-based radiotherapy in patients with head and neck cancer. However, it is essential to know whether the defined hypoxic volume is accurate and extensive enough to target the radioresistant areas. For example, it was shown that all recurrences $(\mathrm{N}=9)$ after chemoradiotherapy were located within the $\left[{ }^{18} \mathrm{~F}\right] \mathrm{FDG}$ avid regions, however 3 of these recurrences were located outside the baseline hypoxic volume, defined on $\left[{ }^{18}\right.$ F]FMISO PET imaging (76). Therefore it is essential to assess the spatial relationship between hypoxic volumes and the recurrences before adapting radiation treatment plans.

Since tumor hypoxia is a dynamic process involving acute and chronic hypoxia, it is of importance to investigate the effect of hypoxia changes during treatment and its effect on the hypoxia PET based radiation treatment plan. A relative stable spatial distribution of the $\left[{ }^{18}\right.$ F]FMISO PET uptake was observed during chemoradiotherapy in 16 patients with locally advanced head and neck cancer (112). While Lin et al. observed a change in the hypoxic volume in 4 out of 7 patients, resulting in decreased radiation coverage based on the initial hypoxia PET based treatment plan (113). Nevertheless, hypoxia dose painting still increased the equivalent uniform dose to the hypoxic 
volume. This dose could however be further increased by incorporating serial hypoxia PET imaging in the radiotherapy plan. These results were confirmed by a study using $\left[{ }^{18}\right.$ F]FAZA PET imaging, before and during radiation therapy, here also fluctuations of hypoxia were observed during treatment and adaptive radiotherapy was recommended (101).

Besides the use for radiotherapy, hypoxia PET imaging could provide useful information for the selection of patients for hypoxia-targeted drugs. The use of $\left[{ }^{18} \mathrm{~F}\right] \mathrm{FMISO}$ during hypoxia targeted therapy with tirapazamine in combination with chemoradiation was investigated in 16 patients with head and neck cancer (114). All tumor sites showed a reduced uptake after 4 weeks of treatment with chemoradiation and tirapazamine, which was consistent with an effect after hypoxia-targeted therapy. These results were confirmed by a later study which showed that $\left[{ }^{18} \mathrm{~F}\right] \mathrm{FMISO}$ PET imaging is associated with a higher risk of locoregional failure in patients treated with (standard) chemo-radiation. In patients with a hypoxic tumor the locoregional control could be improved by additional treatment with tirapazamine, while in patients without tumor hypoxia additional therapy with tirapazamine was not effective. This study demonstrates that $\left[{ }^{18} \mathrm{~F}\right] \mathrm{FMISO}$ may identify patients who may benefit from hypoxia targeting drugs (82).

At the moment there is no literature available regarding the use of hypoxia PET imaging with nitroimidazoles for hypoxia targeting in patients with lung cancer. However there is an ongoing trial investigating the effect of nitroglycerin (a nitric oxide donor, known as a vasodilating agent) on $\left[{ }^{18} \mathrm{~F}\right] \mathrm{HX} 4$ hypoxia PET imaging (NCT01210378). This trial is an example of a new interesting approach of 'window-ofopportunity' clinical trials, to gain insights about the antitumor activity of nitroglycerin or for example other hypoxia modifiers in a disease state not disturbed by previous or simultaneous treatments. In addition a terminated trial is reported (NCT00862134), where patients were treated with the hypoxia activated drug PR104, in combination with $\left[{ }^{18} \mathrm{~F}\right]$ FMISO PET imaging. For patients with renal cell carcinoma, $\left[{ }^{18} \mathrm{~F}\right] \mathrm{FMISO} \mathrm{PET}$ imaging was used to assess the changes of tumor hypoxia after treatment with sunitinib. In this study patients with baseline tumor hypoxia showed a decreased $\left[{ }^{18} \mathrm{~F}\right] \mathrm{FMISO}$ uptake during treatment with sunitinib, while the $\left[{ }^{18} \mathrm{~F}\right] \mathrm{FMISO}$ uptake remained stable in the non-hypoxic lesions (92).

To our knowledge no other hypoxia-targeted drugs were investigated in combination with hypoxia PET imaging. However, we believe that the addition of hypoxia PET 
imaging to the currently investigated anti-hypoxia treatments is essential. In this way patients and acquired results can be stratified based on the presence of tumor hypoxia, creating the evidence for future patient selection.

\section{Future perspectives}

Several nitroimidazole-based PET tracers have shown their ability not only to reliably measure tumor hypoxia but also demonstrated the prognostic significance for treatment outcome in several cancers. Preclinical and clinical studies confirm a value of PET in monitoring important changes in tumor hypoxia during treatment and show the feasibility of integrating hypoxia PET imaging for patient's stratification, therapy optimization before initiation of treatment and/or during therapy. Although the results of the clinical studies incorporating hypoxia PET imaging are promising, none of the hypoxia PET tracers have yet been adequately validated to justify its use in routine clinical practice. Prospective randomized clinical trials integrating hypoxia PET imaging, testing relevant interventions and evaluating dose escalation strategies are therefore necessary.

\section{Acknowledgement}

Authors acknowledge financial support from the QulC-ConCePT project, which is partly funded by EFPI A companies and the Innovative Medicine Initiative Joint Undertaking (IMI JU) under Grant Agreement No. 115151. Authors also acknowledge financial support from the National Institute of Health (NIH-USA U01 CA 143062-01, Radiomics of NSCLC), EU 6th (Euroxy no: 502932) and 7th framework program (Metoxia no: 222741 and ARTFORCE no: 257144), euroCAT (IVA Interreg - www.eurocat.info), Kankeronderzoekfonds Limburg from the Health Foundation Limburg and the Dutch Cancer Society (KWF UM 2008-4210, KWF UM 2009-4454, KWF UM 2011-5020, KWF MAC 2011-4970 , KWF UM 2012-5394, KWF MAC 2013-6425). 


\section{References}

1. Nordsmark M, Overgaard J. A confirmatory prognostic study on oxygenation status and loco-regional control in advanced head and neck squamous cell carcinoma treated by radiation therapy. Radiother Oncol. 2000;57:39-43.

2. Rasey JS, Koh WJ, Evans ML, Peterson LM, Lewellen TK, Graham MM, et al. Quantifying regional hypoxia in human tumors with positron emission tomography of [18F]fluoromisonidazole: a pretherapy study of 37 patients. Int J Radiat Oncol Biol Phys. 1996;36:417-28.

3. Krohn KA, Link JM, Mason RP. Molecular imaging of hypoxia. J Nucl Med. 2008;49 Suppl 2:129S-48S.

4. Gronroos T, Eskola O, Lehtio K, Minn H, Marjamaki P, Bergman J, et al. Pharmacokinetics of [18F]FETNIM: a potential marker for PET. J Nucl Med. 2001;42:1397-404.

5. Lord EM, Harwell L, Koch CJ. Detection of Hypoxic Cells by Monoclonal-Antibody Recognizing 2Nitroimidazole Adducts. Cancer Res. 1993;53:5721-6.

6. Dolbier WR, Jr., Li AR, Koch CJ, Shiue CY, Kachur AV. [18F]-EF5, a marker for PET detection of hypoxia: synthesis of precursor and a new fluorination procedure. Appl Radiat Isot. 2001;54:73-80.

7. Kolb HC, Finn MG, Sharpless KB. Click Chemistry: Diverse Chemical Function from a Few Good Reactions. Angew Chem Int Ed Engl. 2001;40:2004-21.

8. Doss M, Zhang JJ, Belanger MJ, Stubbs JB, Hostetler ED, Alpaugh K, et al. Biodistribution and radiation dosimetry of the hypoxia marker 18F-HX4 in monkeys and humans determined by using whole-body PET/CT. Nucl Med Commun. 2010;31:1016-24.

9. Lopci E, Grassi I, Chiti A, Nanni C, Cicoria G, Toschi L, et al. PET radiopharmaceuticals for imaging of tumor hypoxia: a review of the evidence. Am J Nucl Med Mol Imaging. 2014;4:365-84.

10. Piert $M$, Machulla $H$, Becker $G$, Stahlschmidt A, Patt $M$, Aldinger $P$, et al. Introducing fluorine-18 fluoromisonidazole positron emission tomography for the localisation and quantification of pig liver hypoxia. Eur J Nucl Med. 1999;26:95-109.

11. Bentzen L, Keiding S, Horsman MR, Gronroos T, Hansen SB, Overgaard J. Assessment of hypoxia in experimental mice tumours by [18F]fluoromisonidazole PET and pO2 electrode measurements. Influence of tumour volume and carbogen breathing. Acta Oncol. 2002;41:304-12.

12. Dubois L, Landuyt W, Haustermans K, Dupont P, Bormans G, Vermaelen P, et al. Evaluation of hypoxia in an experimental rat tumour model by [(18)F]fluoromisonidazole PET and immunohistochemistry. Brit J Cancer. 2004;91:1947-54.

13. Troost EG, Laverman P, Philippens ME, Lok J, van der Kogel AJ, Oyen WJ, et al. Correlation of [18F]FMISO autoradiography and pimonidazole [corrected] immunohistochemistry in human head and neck carcinoma xenografts. Eur J Nucl Med Mol Imaging. 2008;35:1803-11.

14. Cho H, Ackerstaff E, Carlin S, Lupu ME, Wang Y, Rizwan A, et al. Noninvasive multimodality imaging of the tumor microenvironment: registered dynamic magnetic resonance imaging and positron emission tomography studies of a preclinical tumor model of tumor hypoxia. Neoplasia. 2009;11:247-59, 2p following 59.

15. Huang T, Civelek AC, Zheng H, Ng CK, Duan X, Li J, et al. (18)F-misonidazole PET imaging of hypoxia in micrometastases and macroscopic xenografts of human non-small cell lung cancer: a correlation with autoradiography and histological findings. Am J Nucl Med Mol Imaging. 2013;3:142-53.

16. Matsumoto K, Szajek L, Krishna MC, Cook JA, Seidel J, Grimes K, et al. The influence of tumor oxygenation on hypoxia imaging in murine squamous cell carcinoma using [64Cu]Cu-ATSM or [18F]Fluoromisonidazole positron emission tomography. Int J Oncol. 2007;30:873-81.

17. Bruehlmeier M, Kaser-Hotz B, Achermann R, Bley CR, Wergin M, Schubiger PA, et al. Measurement of tumor hypoxia in spontaneous canine sarcomas. Vet Radiol Ultrasound. 2005;46:348-54. 
18. O'Donoghue JA, Zanzonico P, Pugachev A, Wen B, Smith-Jones P, Cai S, et al. Assessment of regional tumor hypoxia using 18F-fluoromisonidazole and 64Cu(II)-diacetyl-bis(N4-methylthiosemicarbazone) positron emission tomography: Comparative study featuring microPET imaging, Po2 probe measurement, autoradiography, and fluorescent microscopy in the R3327-AT and FaDu rat tumor models. Int J Radiat Oncol Biol Phys. 2005;61:1493-502.

19. Chang J, Wen B, Kazanzides P, Zanzonico P, Finn RD, Fichtinger G, et al. A robotic system for 18 F-FMISO PET-guided intratumoral pO2 measurements. Med Phys. 2009;36:5301-9.

20. Monnich D, Troost EG, Kaanders JH, Oyen WJ, Alber M, Zips D, et al. Correlation between tumor oxygenation and 18F-fluoromisonidazole PET data simulated based on microvessel images. Acta Oncol. 2013;52:1308-13.

21. Mortensen LS, Buus S, Nordsmark M, Bentzen L, Munk OL, Keiding S, et al. Identifying hypoxia in human tumors: A correlation study between 18F-FMISO PET and the Eppendorf oxygen-sensitive electrode. Acta Oncol. 2010;49:934-40.

22. Gagel B, Piroth M, Pinkawa M, Reinartz P, Zimny M, Kaiser HJ, et al. pO polarography, contrast enhanced color duplex sonography (CDS), [18F] fluoromisonidazole and [18F] fluorodeoxyglucose positron emission tomography: validated methods for the evaluation of therapy-relevant tumor oxygenation or only bricks in the puzzle of tumor hypoxia? BMC Cancer. 2007;7:113.

23. Zimny M, Gagel B, DiMartino E, Hamacher K, Coenen HH, Westhofen M, et al. FDG--a marker of tumour hypoxia? A comparison with [18F]fluoromisonidazole and pO2-polarography in metastatic head and neck cancer. Eur J Nucl Med Mol Imaging. 2006;33:1426-31.

24. Bentzen L, Keiding S, Nordsmark M, Falborg L, Hansen SB, Keller J, et al. Tumour oxygenation assessed by $18 \mathrm{~F}$-fluoromisonidazole PET and polarographic needle electrodes in human soft tissue tumours. Radiother Oncol. 2003;67:339-44.

25. Norikane T, Yamamoto Y, Maeda Y, Kudomi N, Matsunaga T, Haba R, et al. Correlation of (18)Ffluoromisonidazole PET findings with HIF-1alpha and p53 expressions in head and neck cancer: comparison with (18)F-FDG PET. Nucl Med Commun. 2014;35:30-5.

26. Sato J, Kitagawa Y, Yamazaki Y, Hata H, Asaka T, Miyakoshi M, et al. Advantage of FMISO-PET over FDGPET for predicting histological response to preoperative chemotherapy in patients with oral squamous cell carcinoma. Eur J Nucl Med Mol Imaging. 2014.

27. Cherk MH, Foo SS, Poon AM, Knight SR, Murone C, Papenfuss AT, et al. Lack of correlation of hypoxic cell fraction and angiogenesis with glucose metabolic rate in non-small cell lung cancer assessed by 18F-Fluoromisonidazole and 18F-FDG PET. J Nucl Med. 2006;47:1921-6.

28. Cher LM, Murone C, Lawrentschuk N, Ramdave S, Papenfuss A, Hannah A, et al. Correlation of hypoxic cell fraction and angiogenesis with glucose metabolic rate in gliomas using 18F-fluoromisonidazole, 18F-FDG PET, and immunohistochemical studies. J Nucl Med. 2006;47:410-8.

29. Cheng J, Lei L, Xu J, Sun Y, Zhang Y, Wang X, et al. 18F-fluoromisonidazole PET/CT: a potential tool for predicting primary endocrine therapy resistance in breast cancer. J Nucl Med. 2013;54:333-40.

30. Lawrentschuk N, Poon AM, Foo SS, Putra LG, Murone C, Davis ID, et al. Assessing regional hypoxia in human renal tumours using 18F-fluoromisonidazole positron emission tomography. BJU Int. 2005;96:540-6.

31. Busk M, Horsman MR, Jakobsen S, Bussink J, van der Kogel A, Overgaard J. Cellular uptake of PET tracers of glucose metabolism and hypoxia and their linkage. Eur J Nucl Med Mol Imaging. 2008;35:2294-303.

32. Busk M, Horsman MR, Jakobsen S, Keiding S, van der Kogel AJ, Bussink J, et al. Imaging hypoxia in xenografted and murine tumors with 18F-fluoroazomycin arabinoside: a comparative study involving microPET, autoradiography, PO2-polarography, and fluorescence microscopy. Int J Radiat Oncol Biol Phys. 2008;70:1202-12. 
33. Busk M, Horsman MR, Jakobsen S, Hansen KV, Bussink J, van der Kogel A, et al. Can hypoxia-PET map hypoxic cell density heterogeneity accurately in an animal tumor model at a clinically obtainable image contrast? Radiother Oncol. 2009;92:429-36.

34. Busk M, Toustrup K, Sorensen BS, Alsner J, Horsman MR, Jakobsen S, et al. In vivo identification and specificity assessment of mRNA markers of hypoxia in human and mouse tumors. BMC Cancer. 2011;11:63.

35. Carlin S, Zhang H, Reese M, Ramos NN, Chen Q, Ricketts SA. A comparison of the imaging characteristics and microregional distribution of 4 hypoxia PET tracers. J Nucl Med. 2014;55:515-21.

36. Tran LB, Bol A, Labar D, Jordan B, Magat J, Mignion L, et al. Hypoxia imaging with the nitroimidazole 18F-FAZA PET tracer: a comparison with OxyLite, EPR oximetry and 19F-MRI relaxometry. Radiother Oncol. 2012;105:29-35.

37. Lo Dico A, Valtorta S, Martelli C, Belloli S, Gianelli U, Tosi D, et al. Validation of an engineered cell model for in vitro and in vivo HIF-1alpha evaluation by different imaging modalities. Mol Imaging Biol. 2014;16:210-23.

38. Yang DJ, Wallace S, Cherif A, Li C, Gretzer MB, Kim EE, et al. Development of F-18-labeled fluoroerythronitroimidazole as a PET agent for imaging tumor hypoxia. Radiology. 1995;194:795-800.

39. Hu M, Xing L, Mu D, Yang W, Yang G, Kong L, et al. Hypoxia imaging with 18Ffluoroerythronitroimidazole integrated $\mathrm{PET} / \mathrm{CT}$ and immunohistochemical studies in non-small cell lung cancer. Clin Nucl Med. 2013;38:591-6.

40. Chitneni SK, Bida GT, Dewhirst MW, Zalutsky MR. A simplified synthesis of the hypoxia imaging agent 2(2-Nitro-1H-imidazol-1-yl)-N-(2,2,3,3,3-[(18)F]pentafluoropropyl)-acetamide ([18F]EF5). Nucl Med Biol. 2012;39:1012-8.

41. Ziemer LS, Evans SM, Kachur AV, Shuman AL, Cardi CA, Jenkins WT, et al. Noninvasive imaging of tumor hypoxia in rats using the 2-nitroimidazole 18F-EF5. Eur J Nucl Med Mol Imaging. 2003;30:259-66.

42. Chitneni SK, Bida GT, Zalutsky MR, Dewhirst MW. Comparison of the Hypoxia PET Tracer 18 F-EF5 to Immunohistochemical Marker EF5 in 3 Different Human Tumor Xenograft Models. J Nucl Med. 2014;55:1192-7.

43. Silen J, Hogel H, Kivinen K, Silvoniemi A, Forsback S, Loyttyniemi E, et al. Uptake of [F]EF5 as a Tracer for Hypoxic and Aggressive Phenotype in Experimental Head and Neck Squamous Cell Carcinoma. Transl Oncol. 2014.

44. Mahy P, De Bast M, Leveque PH, Gillart J, Labar D, Marchand J, et al. Preclinical validation of the hypoxia tracer 2-(2-nitroimidazol-1-yl)- N-(3,3,3-[(18)F]trifluoropropyl)acetamide, [(18)F]EF3. Eur J Nucl Med Mol Imaging. 2004;31:1263-72.

45. Mahy P, De Bast M, Gillart J, Labar D, Gregoire V. Detection of tumour hypoxia: comparison between EF5 adducts and [18F]EF3 uptake on an individual mouse tumour basis. Eur J Nucl Med Mol Imaging. 2006;33:553-6.

46. Christian N, Bol A, De Bast M, Labar D, Lee J, Mahy P, et al. Determination of tumour hypoxia with the PET tracer [18F]EF3: improvement of the tumour-to-background ratio in a mouse tumour model. Eur J Nucl Med Mol Imaging. 2007;34:1348-54.

47. Dubois LJ, Lieuwes NG, Janssen MH, Peeters WJ, Windhorst AD, Walsh JC, et al. Preclinical evaluation and validation of [18F]HX4, a promising hypoxia marker for PET imaging. Proc Natl Acad Sci U S A. 2011;108:14620-5.

48. Rademakers SE, Lok J, van der Kogel AJ, Bussink J, Kaanders JH. Metabolic markers in relation to hypoxia; staining patterns and colocalization of pimonidazole, HIF-1alpha, CAIX, LDH-5, GLUT-1, MCT1 and MCT4. BMC Cancer. 2011;11:167. 
49. Sorger D, Patt M, Kumar P, Wiebe LI, Barthel H, Seese A, et al. [18F]Fluoroazomycinarabinofuranoside (18FAZA) and [18F]Fluoromisonidazole (18FMISO): a comparative study of their selective uptake in hypoxic cells and PET imaging in experimental rat tumors. Nucl Med Biol. 2003;30:317-26.

50. Piert M, Machulla HJ, Picchio M, Reischl G, Ziegler S, Kumar P, et al. Hypoxia-specific tumor imaging with 18F-fluoroazomycin arabinoside. J Nucl Med. 2005;46:106-13.

51. Reischl G, Dorow DS, Cullinane C, Katsifis A, Roselt P, Binns D, et al. Imaging of tumor hypoxia with [124I]IAZA in comparison with [18F]FMISO and [18F]FAZA--first small animal PET results. J Pharm Pharm Sci. 2007;10:203-11.

52. Maier FC, Kneilling M, Reischl G, Cay F, Bukala D, Schmid A, et al. Significant impact of different oxygen breathing conditions on noninvasive in vivo tumor-hypoxia imaging using [(1)(8)F]-fluoroazomycinarabino-furanoside ([(1)(8)F]FAZA). Radiat Oncol. 2011;6:165.

53. Gronroos T, Bentzen L, Marjamaki P, Murata R, Horsman MR, Keiding S, et al. Comparison of the biodistribution of two hypoxia markers [18F]FETNIM and [18F]FMISO in an experimental mammary carcinoma. Eur J Nucl Med Mol Imaging. 2004;31:513-20.

54. Dubois L, Landuyt W, Cloetens L, Bol A, Bormans G, Haustermans K, et al. [18F]EF3 is not superior to [18F]FMISO for PET-based hypoxia evaluation as measured in a rat rhabdomyosarcoma tumour model. Eur J Nucl Med Mol Imaging. 2009;36:209-18.

55. Mahy P, De Bast M, de Groot T, Cheguillaume A, Gillart J, Haustermans K, et al. Comparative pharmacokinetics, biodistribution, metabolism and hypoxia-dependent uptake of [18F]-EF3 and [18F]MISO in rodent tumor models. Radiother Oncol. 2008;89:353-60.

56. Peeters SG, Zegers CM, Lieuwes NG, Van Elmpt W, Eriksson J, Van Dongen GA, et al. A comparative study of [18F]HX4, [18F]FAZA and [18F]FMISO hypoxia PET tracers in a preclinical

tumor model. Int J Radiat Oncol Biol Phys. 2014.

57. Chen L, Zhang Z, Kolb HC, Walsh JC, Zhang J, Guan Y. (1)(8)F-HX4 hypoxia imaging with PET/CT in head and neck cancer: a comparison with (1)(8)F-FMISO. Nucl Med Commun. 2012;33:1096-102.

58. Postema EJ, McEwan AJ, Riauka TA, Kumar P, Richmond DA, Abrams DN, et al. Initial results of hypoxia imaging using 1-alpha-D: -(5-deoxy-5-[18F]-fluoroarabinofuranosyl)-2-nitroimidazole ( 18F-FAZA). Eur J Nucl Med Mol Imaging. 2009;36:1565-73.

59. Trinkaus ME, Blum R, Rischin D, Callahan J, Bressel M, Segard T, et al. Imaging of hypoxia with 18F-FAZA PET in patients with locally advanced non-small cell lung cancer treated with definitive chemoradiotherapy. J Med Imaging Radiat Oncol. 2013;57:475-81.

60. Zegers $C M$, van Elmpt W, Wierts R, Reymen B, Sharifi H, Ollers MC, et al. Hypoxia imaging with [(1)(8)F]HX4 PET in NSCLC patients: defining optimal imaging parameters. Radiother Oncol. 2013;109:58-64.

61. Szeto MD, Chakraborty G, Hadley J, Rockne R, Muzi M, Alvord EC, Jr., et al. Quantitative metrics of net proliferation and invasion link biological aggressiveness assessed by MRI with hypoxia assessed by FMISO-PET in newly diagnosed glioblastomas. Cancer Res. 2009;69:4502-9.

62. Hirata K, Terasaka S, Shiga T, Hattori N, Magota K, Kobayashi H, et al. (1)(8)F-Fluoromisonidazole positron emission tomography may differentiate glioblastoma multiforme from less malignant gliomas. Eur J Nucl Med Mol Imaging. 2012;39:760-70.

63. Yamamoto $\mathrm{Y}$, Maeda $\mathrm{Y}$, Kawai N, Kudomi N, Aga F, Ono $\mathrm{Y}$, et al. Hypoxia assessed by 18Ffluoromisonidazole positron emission tomography in newly diagnosed gliomas. Nucl Med Commun. 2012;33:621-5.

64. Segard T, Robins PD, Yusoff IF, Ee H, Morandeau L, Campbell EM, et al. Detection of hypoxia with 18Ffluoromisonidazole (18F-FMISO) PET/CT in suspected or proven pancreatic cancer. Clin Nucl Med. 2013;38:1-6. 
65. Loi S, Ngan SY, Hicks RJ, Mukesh B, Mitchell P, Michael M, et al. Oxaliplatin combined with infusional 5fluorouracil and concomitant radiotherapy in inoperable and metastatic rectal cancer: a phase I trial. Brit J Cancer. 2005;92:655-61.

66. Havelund BM, Holdgaard PC, Rafaelsen SR, Mortensen LS, Theil J, Bender D, et al. Tumour hypoxia imaging with 18F-fluoroazomycinarabinofuranoside $\mathrm{PET} / \mathrm{CT}$ in patients with locally advanced rectal cancer. Nucl Med Commun. 2013;34:155-61.

67. Garcia-Parra R, Wood D, Shah RB, Siddiqui J, Hussain H, Park H, et al. Investigation on tumor hypoxia in resectable primary prostate cancer as demonstrated by 18F-FAZA PET/CT utilizing multimodality fusion techniques. Eur J Nucl Med Mol Imaging. 2011;38:1816-23.

68. Bartlett RM, Beattie BJ, Naryanan M, Georgi JC, Chen Q, Carlin SD, et al. Image-guided PO2 probe measurements correlated with parametric images derived from 18F-fluoromisonidazole small-animal PET data in rats. J Nucl Med. 2012;53:1608-15.

69. Busk M, Munk OL, Jakobsen S, Wang T, Skals M, Steiniche T, et al. Assessing hypoxia in animal tumor models based on pharmocokinetic analysis of dynamic FAZA PET. Acta Oncol. 2010;49:922-33.

70. Wang W, Lee NY, Georgi JC, Narayanan M, Guillem J, Schoder H, et al. Pharmacokinetic analysis of hypoxia (18)F-fluoromisonidazole dynamic PET in head and neck cancer. J Nucl Med. 2010;51:37-45.

71. Thorwarth D, Eschmann SM, Paulsen F, Alber M. A kinetic model for dynamic [18F]-Fmiso PET data to analyse tumour hypoxia. Phys Med Biol. 2005;50:2209-24.

72. Verwer EE, van Velden FH, Bahce I, Yaqub M, Schuit RC, Windhorst AD, et al. Pharmacokinetic analysis of [18F]FAZA in non-small cell lung cancer patients. Eur J Nucl Med Mol Imaging. 2013;40:1523-31.

73. Mortensen LS, Busk M, Nordsmark M, Jakobsen S, Theil J, Overgaard J, et al. Accessing radiation response using hypoxia PET imaging and oxygen sensitive electrodes: a preclinical study. Radiother Oncol. 2011;99:418-23.

74. Beck R, Roper B, Carlsen JM, Huisman MC, Lebschi JA, Andratschke N, et al. Pretreatment 18F-FAZA PET predicts success of hypoxia-directed radiochemotherapy using tirapazamine. J Nucl Med. 2007;48:97380.

75. Lehtio K, Eskola O, Viljanen T, Oikonen V, Gronroos T, Sillanmaki L, et al. Imaging perfusion and hypoxia with PET to predict radiotherapy response in head-and-neck cancer. Int J Radiat Oncol Biol Phys. 2004;59:971-82.

76. Dirix P, Vandecaveye V, De Keyzer F, Stroobants S, Hermans R, Nuyts S. Dose painting in radiotherapy for head and neck squamous cell carcinoma: value of repeated functional imaging with (18)F-FDG PET, (18)F-fluoromisonidazole PET, diffusion-weighted MRI, and dynamic contrast-enhanced MRI. J Nucl Med. 2009;50:1020-7.

77. Mortensen LS, Johansen J, Kallehauge J, Primdahl H, Busk M, Lassen P, et al. FAZA PET/CT hypoxia imaging in patients with squamous cell carcinoma of the head and neck treated with radiotherapy: results from the DAHANCA 24 trial. Radiother Oncol. 2012;105:14-20.

78. Lee N, Nehmeh S, Schoder H, Fury M, Chan K, Ling CC, et al. Prospective trial incorporating pre-/midtreatment [18F]-misonidazole positron emission tomography for head-and-neck cancer patients undergoing concurrent chemoradiotherapy. Int J Radiat Oncol Biol Phys. 2009;75:101-8.

79. Zips D, Zophel K, Abolmaali N, Perrin R, Abramyuk A, Haase R, et al. Exploratory prospective trial of hypoxia-specific PET imaging during radiochemotherapy in patients with locally advanced head-andneck cancer. Radiother Oncol. 2012;105:21-8.

80. Eschmann SM, Paulsen F, Bedeshem C, Machulla HJ, Hehr T, Bamberg M, et al. Hypoxia-imaging with (18)F-Misonidazole and PET: changes of kinetics during radiotherapy of head-and-neck cancer. Radiother Oncol. 2007;83:406-10. 
81. Thorwarth D, Eschmann SM, Scheiderbauer J, Paulsen F, Alber M. Kinetic analysis of dynamic 18Ffluoromisonidazole PET correlates with radiation treatment outcome in head-and-neck cancer. BMC Cancer. 2005;5:152.

82. Rischin D, Hicks RJ, Fisher R, Binns D, Corry J, Porceddu S, et al. Prognostic significance of [18F]misonidazole positron emission tomography-detected tumor hypoxia in patients with advanced head and neck cancer randomly assigned to chemoradiation with or without tirapazamine: a substudy of Trans-Tasman Radiation Oncology Group Study 98.02. J Clin Oncol. 2006;24:2098-104.

83. Rajendran JG, Schwartz DL, O'Sullivan J, Peterson LM, Ng P, Scharnhorst J, et al. Tumor hypoxia imaging with [F-18] fluoromisonidazole positron emission tomography in head and neck cancer. Clin Cancer Res. 2006;12:5435-41.

84. Komar G, Lehtio K, Seppanen M, Eskola O, Levola H, Lindholm P, et al. Prognostic value of tumour blood flow, [(18)F]EF5 and [ (18)F]FDG PET/CT imaging in patients with head and neck cancer treated with radiochemotherapy. Eur J Nucl Med Mol Imaging. 2014;41:2042-50.

85. Thorwarth D, Eschmann SM, Holzner F, Paulsen F, Alber M. Combined uptake of [18F]FDG and [18F]FMISO correlates with radiation therapy outcome in head-and-neck cancer patients. Radiother Oncol. 2006;80:151-6.

86. Li L, Hu M, Zhu H, Zhao W, Yang G, Yu J. Comparison of 18F-Fluoroerythronitroimidazole and 18Ffluorodeoxyglucose positron emission tomography and prognostic value in locally advanced non-smallcell lung cancer. Clin Lung Cancer. 2010;11:335-40.

87. Eschmann SM, Paulsen F, Reimold M, Dittmann H, Welz S, Reischl G, et al. Prognostic impact of hypoxia imaging with 18F-misonidazole PET in non-small cell lung cancer and head and neck cancer before radiotherapy. J Nucl Med. 2005;46:253-60.

88. Kawai N, Lin W, Cao WD, Ogawa D, Miyake K, Haba R, et al. Correlation between (18)Ffluoromisonidazole PET and expression of HIF-1alpha and VEGF in newly diagnosed and recurrent malignant gliomas. Eur J Nucl Med Mol Imaging. 2014;41:1870-8.

89. Swanson KR, Chakraborty G, Wang CH, Rockne R, Harpold HL, Muzi M, et al. Complementary but distinct roles for $\mathrm{MRI}$ and 18F-fluoromisonidazole PET in the assessment of human glioblastomas. J Nucl Med. 2009;50:36-44.

90. Vercellino L, Groheux D, Thoury A, Delord M, Schlageter MH, Delpech Y, et al. Hypoxia imaging of uterine cervix carcinoma with (18)F-FETNIM PET/CT. Clin Nucl Med. 2012;37:1065-8.

91. Yue J, Yang Y, Cabrera AR, Sun X, Zhao S, Xie P, et al. Measuring tumor hypoxia with (1)(8)F-FETNIM PET in esophageal squamous cell carcinoma: a pilot clinical study. Dis Esophagus. 2012;25:54-61.

92. Hugonnet F, Fournier L, Medioni J, Smadja C, Hindie E, Huchet V, et al. Metastatic renal cell carcinoma: relationship between initial metastasis hypoxia, change after 1 month's sunitinib, and therapeutic response: an 18F-fluoromisonidazole PET/CT study. J Nucl Med. 2011;52:1048-55.

93. Oehler C, O'Donoghue JA, Russell J, Zanzonico P, Lorenzen S, Ling CC, et al. 18F-fluromisonidazole PET imaging as a biomarker for the response to 5,6-dimethylxanthenone-4-acetic acid in colorectal xenograft tumors. J Nucl Med. 2011;52:437-44.

94. Valable S, Petit E, Roussel S, Marteau L, Toutain J, Divoux D, et al. Complementary information from magnetic resonance imaging and (18)F-fluoromisonidazole positron emission tomography in the assessment of the response to an antiangiogenic treatment in a rat brain tumor model. Nucl Med Biol. 2011;38:781-93.

95. Murakami M, Zhao S, Zhao Y, Chowdhury NF, Yu W, Nishijima K, et al. Evaluation of changes in the tumor microenvironment after sorafenib therapy by sequential histology and 18F-fluoromisonidazole hypoxia imaging in renal cell carcinoma. Int J Oncol. 2012;41:1593-600. 
96. Busk M, Mortensen LS, Nordsmark M, Overgaard J, Jakobsen S, Hansen KV, et al. PET hypoxia imaging with FAZA: reproducibility at baseline and during fractionated radiotherapy in tumour-bearing mice. Eur J Nucl Med Mol Imaging. 2013;40:186-97.

97. Dorow DS, Cullinane C, Conus N, Roselt P, Binns D, McCarthy TJ, et al. Multi-tracer small animal PET imaging of the tumour response to the novel pan-Erb-B inhibitor $\mathrm{Cl}-1033$. Eur J Nucl Med Mol Imaging. 2006;33:441-52.

98. Solomon B, Binns D, Roselt P, Weibe LI, McArthur GA, Cullinane C, et al. Modulation of intratumoral hypoxia by the epidermal growth factor receptor inhibitor gefitinib detected using small animal PET imaging. Mol Cancer Ther. 2005;4:1417-22.

99. Chitneni SK, Bida GT, Yuan H, Palmer GM, Hay MP, Melcher T, et al. 18F-EF5 PET imaging as an early response biomarker for the hypoxia-activated prodrug SN30000 combined with radiation treatment in a non-small cell lung cancer xenograft model. J Nucl Med. 2013;54:1339-46.

100. Yamane T, Kikuchi M, Shinohara S, Senda M. Reduction of [(18)F]fluoromisonidazole uptake after neoadjuvant chemotherapy for head and neck squamous cell carcinoma. Mol Imaging Biol. 2011;13:227-31.

101. Servagi-Vernat S, Differding S, Hanin FX, Labar D, Bol A, Lee JA, et al. A prospective clinical study of (1)(8)F-FAZA PET-CT hypoxia imaging in head and neck squamous cell carcinoma before and during radiation therapy. Eur J Nucl Med Mol Imaging. 2014;41:1544-52.

102. Koh WJ, Bergman KS, Rasey JS, Peterson LM, Evans ML, Graham MM, et al. Evaluation of oxygenation status during fractionated radiotherapy in human nonsmall cell lung cancers using [F18]fluoromisonidazole positron emission tomography. Int J Radiat Oncol Biol Phys. 1995;33:391-8.

103. Vera P, Bohn P, Edet-Sanson A, Salles A, Hapdey S, Gardin I, et al. Simultaneous positron emission tomography (PET) assessment of metabolism with (1)(8)F-fluoro-2-deoxy-d-glucose (FDG), proliferation with (1)(8)F-fluoro-thymidine (FLT), and hypoxia with (1)(8)fluoro-misonidazole (F-miso) before and during radiotherapy in patients with non-small-cell lung cancer (NSCLC): a pilot study. Radiother Oncol. 2011;98:109-16.

104. Schuetz M, Schmid MP, Potter R, Kommata S, Georg D, Lukic D, et al. Evaluating repetitive 18Ffluoroazomycin-arabinoside (18FAZA) PET in the setting of MRI guided adaptive radiotherapy in cervical cancer. Acta Oncol. 2010;49:941-7.

105. Eary JF, Link JM, Muzi M, Conrad EU, Mankoff DA, White JK, et al. Multiagent PET for risk characterization in sarcoma. J Nucl Med. 2011;52:541-6.

106. Rajendran JG, Mankoff DA, O'Sullivan F, Peterson LM, Schwartz DL, Conrad EU, et al. Hypoxia and glucose metabolism in malignant tumors: evaluation by [18F]fluoromisonidazole and [18F]fluorodeoxyglucose positron emission tomography imaging. Clin Cancer Res. 2004;10:2245-52.

107. Overgaard J, Horsman MR. Modification of Hypoxia-Induced Radioresistance in Tumors by the Use of Oxygen and Sensitizers. Semin Radiat Oncol. 1996;6:10-21.

108. Choi W, Lee SW, Park SH, Ryu JS, Oh SJ, Im KC, et al. Planning study for available dose of hypoxic tumor volume using fluorine-18-labeled fluoromisonidazole positron emission tomography for treatment of the head and neck cancer. Radiother Oncol. 2010;97:176-82.

109. Hendrickson K, Phillips M, Smith W, Peterson L, Krohn K, Rajendran J. Hypoxia imaging with [F-18] FMISO-PET in head and neck cancer: potential for guiding intensity modulated radiation therapy in overcoming hypoxia-induced treatment resistance. Radiother Oncol. 2011;101:369-75.

110. Toma-Dasu I, Uhrdin J, Antonovic L, Dasu A, Nuyts S, Dirix P, et al. Dose prescription and treatment planning based on FMISO-PET hypoxia. Acta Oncol. 2012;51:222-30.

111. Chang JH, Wada M, Anderson NJ, Lim Joon D, Lee ST, Gong SJ, et al. Hypoxia-targeted radiotherapy dose painting for head and neck cancer using (18)F-FMISO PET: a biological modeling study. Acta Oncol. 2013;52:1723-9. 
112. Bittner MI, Wiedenmann N, Bucher S, Hentschel M, Mix M, Weber WA, et al. Exploratory geographical analysis of hypoxic subvolumes using 18F-MISO-PET imaging in patients with head and neck cancer in the course of primary chemoradiotherapy. Radiother Oncol. 2013;108:511-6.

113. Lin Z, Mechalakos J, Nehmeh S, Schoder H, Lee N, Humm J, et al. The influence of changes in tumor hypoxia on dose-painting treatment plans based on 18F-FMISO positron emission tomography. Int J Radiat Oncol Biol Phys. 2008;70:1219-28.

114. Hicks RJ, Rischin D, Fisher R, Binns D, Scott AM, Peters LJ. Utility of FMISO PET in advanced head and neck cancer treated with chemoradiation incorporating a hypoxia-targeting chemotherapy agent. Eur J Nucl Med Mol Imaging. 2005;32:1384-91. 


\section{Chapter 3}

\section{A comparative study of the hypoxia PET tracers $\left[{ }^{18} \mathrm{~F}\right] \mathrm{HX} 4,\left[{ }^{18} \mathrm{~F}\right] \mathrm{FAZA}$ and $\left[{ }^{18} \mathrm{~F}\right] \mathrm{FMISO}$ in a preclinical tumor model}

Published in: Int J Radiat Oncol Biol Phys. 2015;91:351-9

Sarah G.J.A. Peeters, Catharina M.L. Zegers, Natasja G. Lieuwes, Wouter van Elmpt, Jonas Eriksson, Guus A.M.S. van Dongen, Ludwig Dubois*, Philippe Lambin* 


\section{Summary}

Visualizing hypoxic tumor regions is of importance for improving cancer treatment and hypoxia positron emission tomography has been shown to be a promising tool. The tumor uptake of 3 clinical hypoxia PET tracers, $\left[{ }^{18} \mathrm{~F}\right] \mathrm{FMISO},\left[{ }^{18} \mathrm{~F}\right] \mathrm{FAZA}$ and $\left[{ }^{18} \mathrm{~F}\right] \mathrm{HX} 4$, was assessed within 1 preclinical tumor model to compare their performances regarding optimal imaging time, tumor-to-background ratios, spatial reproducibility and modified oxygen concentrations. This study provides insights into the strengths and weaknesses of these tracers.

\section{Abstract}

Purpose: Several individual clinical and preclinical studies have shown the possibility to evaluate tumor hypoxia by using noninvasive positron emission tomography (PET). The current study compared 3 hypoxia PET tracers frequently used in the clinic, $\left[{ }^{18} \mathrm{~F}\right] \mathrm{FMISO}$, $\left[{ }^{18} \mathrm{~F}\right] \mathrm{FAZA}$ and $\left[{ }^{18} \mathrm{~F}\right] \mathrm{HX} 4$ in a preclinical tumor model. Tracer uptake was evaluated for the optimal time point for imaging, tumor-to-blood ratios (TBR), spatial reproducibility and sensitivity towards oxygen modifications.

Methods and Materials: PET/computed tomotgraphy (CT) images of rhabdomyosarcoma R1 bearing WAG/Rij rats were acquired at multiple time points post injection (p.i.) with one of the hypoxia tracers. TBR values were calculated and reproducibility was investigated by voxel-to-voxel analysis, represented as correlation coefficients (R) or DICE similarity coefficient of the high-uptake volumes. Tumor oxygen modifications were induced by exposure to either carbogen/nicotinamide treatment or $7 \%$ oxygen breathing.

Results: TBR was stabilized and maximal at 2 hours p.i. for $\left[{ }^{18} \mathrm{~F}\right] \mathrm{FAZA}(4.0 \pm 0.5)$ and at 3 hours p.i. for $\left[{ }^{18} \mathrm{~F}\right] \mathrm{HX} 4(7.2 \pm 0.7)$ whereas $\left[{ }^{18} \mathrm{~F}\right] \mathrm{FMISO}$ showed a constant increasing TBR $(9.0 \pm 0.8$ at 6 hours p.i.). High spatial reproducibility was observed by voxel-to-voxel comparisons and DICE similarity coefficient calculations on the $30 \%$ highest uptake volume for both $\left[{ }^{18} \mathrm{~F}\right] \mathrm{FMISO}(\mathrm{R}=0.86$, DICE coefficient $=0.76)$ and $\left[{ }^{18} \mathrm{~F}\right] \mathrm{HX} 4(\mathrm{R}=0.76$, DICE coefficient $=0.70)$ whereas $\left[{ }^{18} \mathrm{~F}\right] \mathrm{FAZA}$ was less reproducible $(\mathrm{R}=0.52$ DICE coefficient $=0.49$ ). Modifying the hypoxic fraction resulted in enhanced mean standardized uptake values for both $\left[{ }^{18} \mathrm{~F}\right] \mathrm{HX} 4$ and $\left[{ }^{18} \mathrm{~F}\right] \mathrm{FAZA}$ upon $7 \%$ oxygen breathing. Only $\left[{ }^{18} \mathrm{~F}\right] \mathrm{FMISO}$ uptake was found to be reversible upon exposure to nicotinamide and carbogen.

Conclusions: This study indicates that each tracer has its own strengths and, depending on the question to be answered, a different tracer can be put forward. 


\section{Introduction}

Tumor hypoxia is an important factor in worsening cancer patients' treatment outcome. Regions of low oxygen concentration are a well-known characteristic of solid tumors and can be caused by impaired blood vessel development, temporal occlusions of blood vessels or excessive tumor growth $(1,2)$. Knowledge about the extension and location of hypoxia would provide additional information that could be integrated into strategies of conventional treatments, potentially leading to improved therapeutic outcome (3). Positron Emission Tomography (PET) has been shown to be a suitable, noninvasive, 3-dimensional imaging technique for the detection of hypoxic tumor regions. PET tracers containing the oxygen-sensitive nitroimidazole group are specifically designed to detect hypoxic regions, and the feasibility of these tracers has been studied extensively in several independent clinical and preclinical studies (1).

${ }^{18} \mathrm{~F}$ - labeled fluoromisonidazole ( $\left[{ }^{18} \mathrm{~F}\right] \mathrm{FMISO}$ ) was the first specific hypoxia PET tracer and, although it may be the most frequently used tracer for this purpose, its suitability is limited because of slow tumor-specific accumulation and nonspecific washout (4). Second generation 2-nitroimidazole tracers with different clearance and hydrophilicity characteristics have been developed in an attempt to overcome these disadvantages:, $\left[{ }^{18} \mathrm{~F}\right]$ fluoroazomycin arabinoside (FAZA), $\left[{ }^{18} \mathrm{~F}\right]$ fluoroerythronitroimidazole (FETNIM), and $\left[{ }^{18} \mathrm{~F}\right]$ tri-fluoroetanidazole (EF3). In preclinical settings, these tracers have been investigated separately or solely in comparison to hypoxia immunohistochemical staining or $\left[{ }^{18} \mathrm{~F}\right] \mathrm{FMISO}$ PET imaging, using different experimental set-ups, tumor models and acquisition protocols (5-8). Next, the third-generation hypoxia tracer $\left[{ }^{18} \mathrm{~F}\right]$ flortanidazole $(\mathrm{HX} 4)$ was developed and evaluated in a preclinical rhabdomyosarcoma tumor model, where it was found to be dependent on tumoral oxygenation status (9). Only recently, a comparative study in preclinical animal models reported a clear relationship among the uptake of $\left[{ }^{18} \mathrm{~F}\right] \mathrm{FMISO},\left[{ }^{18} \mathrm{~F}\right] \mathrm{FAZA}$ and $\left[{ }^{18} \mathrm{~F}\right] \mathrm{HX} 4$ and with immunohistochemical stainings for perfusion, hypoxia and carbonic anhydrase IX (10). With respect to patients usage of PET tumor hypoxia for patient imaging, clinical studies have indicated that both $\left[{ }^{18} \mathrm{~F}\right] \mathrm{FAZA}$ and $\left[{ }^{18} \mathrm{~F}\right] \mathrm{FMISO}$ have prognostic potential $(11,12)$ and a phase I clinical study demonstrated that imaging using $\left[{ }^{18} \mathrm{~F}\right] \mathrm{HX} 4$ was feasible and non-toxic (13).

In this study, we compare the 3 most frequently used and clinically available hypoxia tracers, $\left[{ }^{18} \mathrm{~F}\right] \mathrm{FMISO},\left[{ }^{18} \mathrm{~F}\right] \mathrm{FAZA}$ and $\left[{ }^{18} \mathrm{~F}\right] \mathrm{HX} 4$, along with the metabolic tracer $\left[{ }^{18} \mathrm{~F}\right]$ fluorodeoxyglucose (FDG) in a preclinical tumor model. We investigated the uptake of each tracer over time and determined the tumor-to-background ratios. A 
second PET scan was performed on the same animal after 48 hours to assess spatial reproducibility. Furthermore, tracer uptake was challenged by exposing the animals to modified oxygen concentrations. The focus of this study was to investigate the performance and characteristics of the different hypoxia PET tracer, using the same tumor model.

\section{Material and Methods}

\section{Tracer synthesis, tumor model, experimental design}

Tracer synthesis of $\left[{ }^{18} \mathrm{~F}\right] \mathrm{FMISO},\left[{ }^{18} \mathrm{~F}\right] \mathrm{FAZA}$ and $\left[{ }^{18} \mathrm{~F}\right] \mathrm{HX} 4$ (figure 3.1A) was performed as described previously (14-17). All animal experimental procedures were approved by the Animal Ethical Committee of Maastricht University and were in accordance with the Helsinki Declaration of 1975, as revised in 2000. Adult WAG/Rij rats received subcutaneous implants of the syngeneic rhabdomyosarcoma R1 tumors $\left(1 \mathrm{~mm}^{3}\right)$ in the lateral flank. Experiments were started when tumors reached a minimal volume of 3 $\mathrm{cm}^{3}$ to meet the resolution of the PET scanner and to have a stable hypoxic (18) and necrotic (5) area. Average tumor volume for $\left[{ }^{18} \mathrm{~F}\right] \mathrm{FDG}$ is $21 \pm 12 \mathrm{~cm}^{3}, 16 \pm 6 \mathrm{~cm}^{3}$ for $\left[{ }^{18} \mathrm{~F}\right] \mathrm{FAZA}, 13 \pm 6 \mathrm{~cm}^{3}$ for $\left[{ }^{18} \mathrm{~F}\right] \mathrm{FMISO}$ and $11 \pm 5 \mathrm{~cm}^{3}$ for $\left[{ }^{18} \mathrm{~F}\right] \mathrm{HX} 4$. During the experimental procedures, rats were anesthetized with intraperitoneal injections of sodium pentobarbital $(60 \mathrm{mg} / \mathrm{kg})$. Animals were immobilized on a board and placed outside the scanner between scans to maintain and monitor anesthesia. Radioactive tracers (radiochemical purity was maintained at $>95 \%$ and synthesis yield at $5.2 \pm 2.5 \mathrm{GBq}$ ) were injected into the lateral tail vein by using an intravenous line $(0.4 \mathrm{~mm} \mathrm{G} 27$ Venoflux needle; Vygon Vet, Ecouen, France) flushed with $10 \%$ heparin solution $(21 \pm 2$ $\mathrm{MBq}$ for $\left[{ }^{18} \mathrm{~F}\right] \mathrm{FDG}, 17 \pm 5 \mathrm{MBq}$ for $\left[{ }^{18} \mathrm{~F}\right] \mathrm{FAZA}, 21 \pm 2 \mathrm{MBq}$ for $\left[{ }^{18} \mathrm{~F}\right] \mathrm{FMISO}$, and $21 \pm 2 \mathrm{MBq}$ for $\left.\left[{ }^{18} \mathrm{~F}\right] \mathrm{HX} 4\right)$.

\section{Image acquisition and analysis}

Images were acquired and analyzed using a clinical PET/CT scanner (Siemens Biograph 40, Siemens Healthcare) and dedicated software (TrueD VC60; Siemens) as described in more detail in the supplement section and previously (9). Tumor to blood ratios (TBR) and tumor to muscle ratios (TMR) were determined using heart and muscle of the hind leg as background tissue, respectively. Spatial reproducibility scans were performed in the same animal within short time frames, using rigid registration voxel to voxel analysis (from 2 to 6 hours post injection [p.i].) or 48 hours apart using 
nonrigid registration (details in supplement section) of the tumor for long-term comparison to overcome the $24 \%$ tumor growth (which were $31 \pm 2 \%$ for $\left[{ }^{18} \mathrm{~F}\right] \mathrm{FDG}$, $26 \pm 2 \%$ for $\left[{ }^{18} \mathrm{~F}\right] \mathrm{FAZA}, 23 \pm 1 \%$ for $\left[{ }^{18} \mathrm{~F}\right] \mathrm{FMISO}$, and $22 \pm 3 \%$ for $\left.\left[{ }^{18} \mathrm{~F}\right] \mathrm{HX} 4\right)$. Furthermore, a voxel-wise comparison of the 2 scans was performed for which a correlation coefficient was calculated. Imaging schedules for oxygen modification using either nicotinamide $\left(500 \mathrm{mg} / \mathrm{kg}\right.$, intraperitoneal) and carbogen $\left(95 \% \mathrm{O}_{2}, 5 \% \mathrm{CO}_{2}\right.$, flow $\left.5 \mathrm{l} / \mathrm{min}\right)$ or $7 \%$ oxygen (residual $\mathrm{N}_{2}$, flow $2,5 \mathrm{l} / \mathrm{min}$ ) breathing are shown in figure 3.1B. In short, after injection of the tracer, a first basal scan was performed at 2 hours p.i., followed by oxygen modification treatment and a second scan at 5 hours p.i.

A

["F]FMSO<smiles>O=[N+]([O-])c1nccn1CC(O)CF</smiles>

Clearance

Hydrophilicity (logP)

B

Dynamic imaging

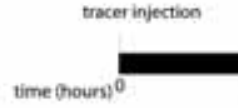

["F]HX4<smiles>O=[N+]([O-])c1nccn1Cc1cnnn1C(CO)CF</smiles>

renal 0.04

renal

$-0.69$
["F]FDG<smiles>OCC1OC(O)C(F)C(O)C1O</smiles>

Oxygen modification

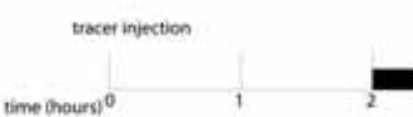

axyen modification
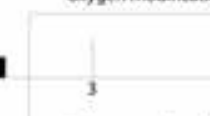

nicotinamide / carbogen breathing

Figure 3.1 Tracer characteristics and imaging protocols A) Structure formula, main clearance system and logP value for hydrophilicity of the 3 hypoxia tracers $\left[{ }^{18} \mathrm{~F}\right] \mathrm{FMISO},\left[{ }^{18} \mathrm{~F}\right] \mathrm{FAZA}$ and $\left[{ }^{18} \mathrm{~F}\right] \mathrm{HX} 4$ and the metabolic tracer $\left[{ }^{18}\right.$ F]FDG (9). B) Schematic representation of the different imaging protocols for dynamic imaging and for oxygen modification, using either nicotinamide combined with carbogen breathing or $7 \%$ oxygen breathing. Imaging acquisition is indicated by black boxes. 
A

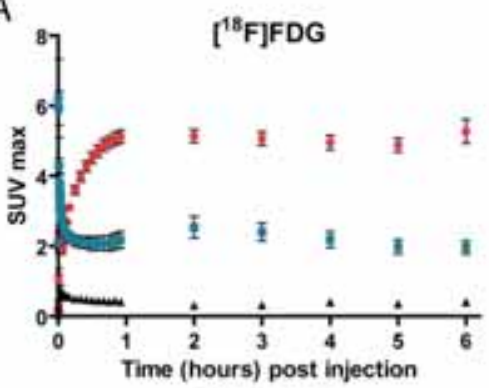

$\left[{ }^{18} \mathrm{~F}\right] \mathrm{FMISO}$

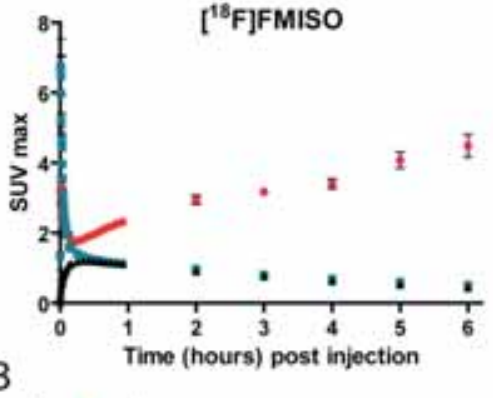

B

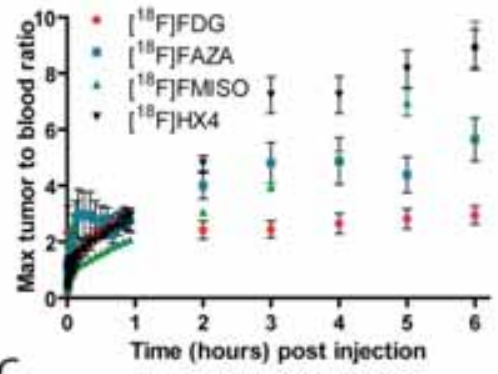

C

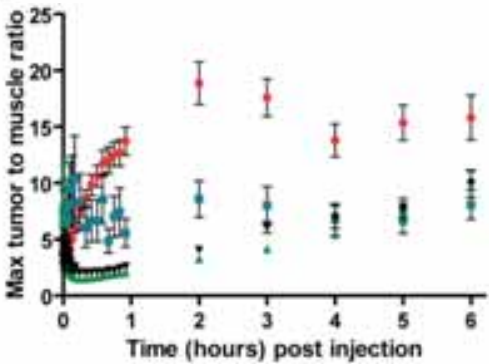

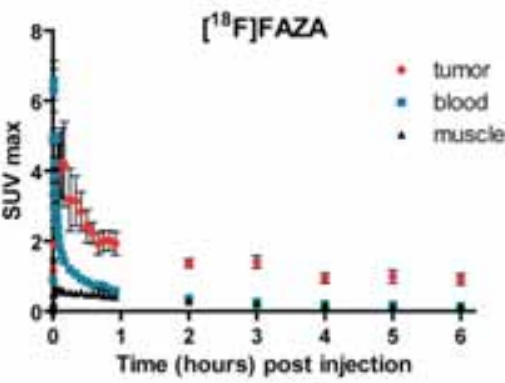

$\left[{ }^{18} \mathrm{~F}\right] \mathrm{HX} 4$
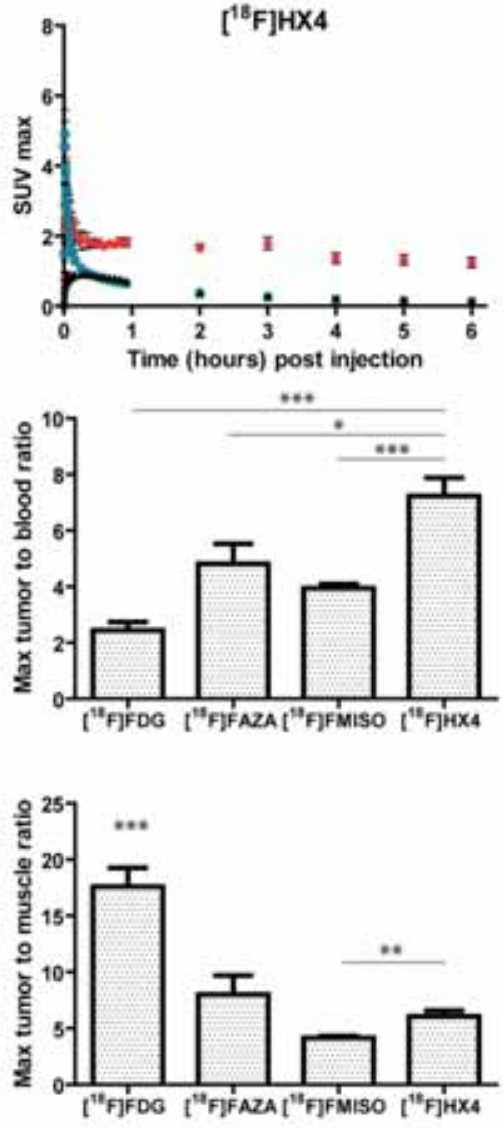

Figure 3.2 SUVmax and TBR A) SUVmax of the tumor (red), blood (blue) and muscle (black) over time. B) Left panel: Maximum TBR over time for $\left[{ }^{18} \mathrm{~F}\right] F \mathrm{FG}$ (red), $\left[{ }^{18} \mathrm{~F}\right] \mathrm{FAZA}$ (blue), $\left[{ }^{18} \mathrm{~F}\right] \mathrm{FMISO}$ (green) and $\left[{ }^{18} \mathrm{~F}\right] \mathrm{HX} 4$ (black). Right panel; TBR tracer comparison at 3 hours post-injection. ${ }^{*} \mathrm{P}<0.05,{ }^{* * *}<0.001$. C) Left panel: Maximum TMR over time. Right panel: Maximum TMR comparison for the 4 tracers at 3 hours p.i. Number of animals for all experimetns: $\left[{ }^{18} \mathrm{~F}\right] \mathrm{FDG} n=12,\left[{ }^{18} \mathrm{~F}\right] F A Z A n=13,\left[{ }^{18} \mathrm{~F}\right] \mathrm{FMISO} n=16$ and $\left[{ }^{18} \mathrm{~F}\right] \mathrm{HX} 4 \mathrm{n}=18$ except for Figure $\mathrm{C}$ $\left[{ }^{18} \mathrm{~F}\right] \mathrm{FAZA} \mathrm{n}=12$. Data are means $\pm \mathrm{SEM}$. SUVmax=maximum standard uptake value; TBR=tumor-to-blood ratio; TMR=tumor-to-muscle ratio. 


\section{Statistics}

Prism version 5.01 software (GraphPad) for Windows (Microsoft) was used to perform statistical analyses. To determine the statistical significance of differences between 2 independent groups of variables, we used the nonparametric Mann-Whitney-U test for small groups. Spatial reproducibility was analyzed using either a DICE similarity coefficient for the calculation of the overlap fractions or Pearson correlation for the voxel wise comparison. P-values of $<0.05$ were assumed to be significant.

\section{Results}

PET/CT imaging was performed to assess tracer accumulation over time for the 4 different tracers, using a dynamic imaging schedule (figure 3.1A, B). Each tracer had a different accumulation pattern within the tumor, represented by maximum (SUV $V_{\max }$ figure 3.2A) and mean (SUV mean figure S3.1A) standardized uptake values, whereas blood and muscle tracer uptake exhibited a similar pattern. Due to clearance of the nonspecific binding and specific trapping in hypoxic tumor regions, all TBRs were greater than 1 (figure 3.2B and figure S3.1B). Furthermore, clearance rates for heart and muscle were comparable, giving a muscle to blood ratio around unity (figure S3.1D). For the metabolic tracer $\left[{ }^{18} \mathrm{~F}\right] \mathrm{FDG}$, the maximal TBR of $2.4 \pm 0.3$ was reached at 2 hours p.i. Of all the hypoxia tracers, $\left[{ }^{18} \mathrm{~F}\right] \mathrm{FAZA}$ was the first to reach a plateau phase for TBR at 2 hours p.i. $(4.0 \pm 0.5)$, followed by $\left[{ }^{18} \mathrm{~F}\right] \mathrm{HX} 4(\mathrm{TBR} 7.2 \pm 0.7)$ at 3 hours p.i. (figure 3.2B). TBR for $\left[{ }^{18} \mathrm{~F}\right] \mathrm{FMISO}$ kept increasing; TBR of $\left[{ }^{18} \mathrm{~F}\right] \mathrm{FMISO}$ at 6 hours p.i. was comparable to that of $\left[{ }^{18} \mathrm{~F}\right] \mathrm{HX} 4$ at 3 hours p.i. At the first stable time point for $\left[{ }^{18} \mathrm{~F}\right] \mathrm{HX} 4$ ( 3 hours p.i.), this tracer had a significantly higher TBR than either $\left[{ }^{18} \mathrm{~F}\right] \mathrm{FAZA}(\mathrm{P}=0.0154)$ or $\left[{ }^{18} \mathrm{~F}\right] \mathrm{FMISO}\left(\mathrm{P}<0.0001\right.$ ) (figure $3.2 \mathrm{~B}$ right panel) even at 2 hours p.i. $\left[{ }^{18} \mathrm{~F}\right] \mathrm{HX} 4$ had already reached a TBR that was equal to or higher than that of $\left[{ }^{18} \mathrm{~F}\right] \mathrm{FMISO}$ or $\left[{ }^{18} \mathrm{~F}\right] \mathrm{FAZA}$. When muscle tissue was used as tissue reference, trends were shown for the hypoxia tracers that were similar to maximal TBR (figure 3.2C and figure S3.1C).

Uptake images from 2, 3, 4 and 5 hours p.i. were compared to the 6 hours p.i. scan to perform a voxel-to-voxel comparison of the absolute tumor uptake. A correlation coefficient was calculated from the 2 scans (see figure 3.3A). Averaged correlation coefficients demonstrated a stable uptake pattern in the tumor for all investigated tracers over short time periods (up to 6 hours) (figure 3.3B). Reproducibility was studied by comparing 2 PET scans acquired within a 48 hour time interval using voxelto-voxel analyses. Calculated correlation coefficients were high for $\left[{ }^{18} \mathrm{~F}\right] \mathrm{FDG}(0.87)$ 
A

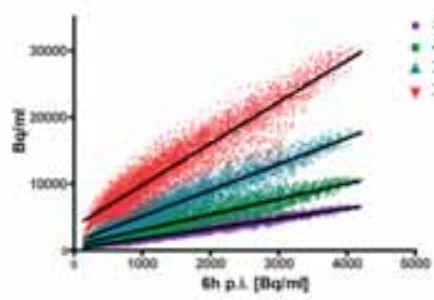

C

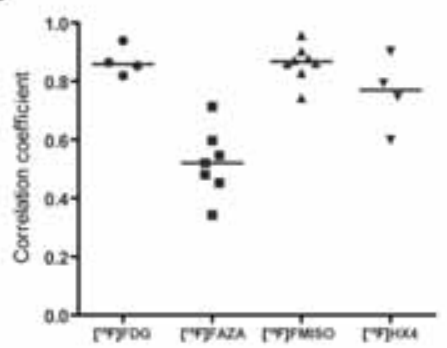

B
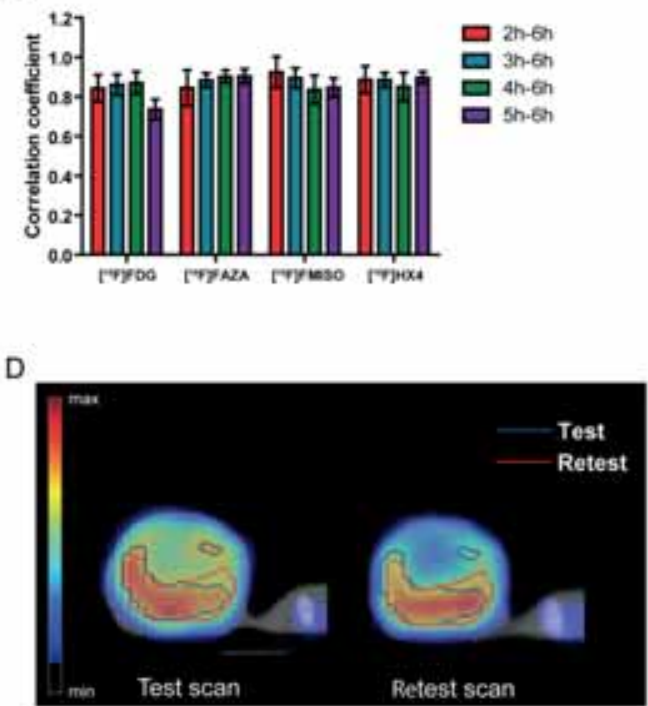

$\mathrm{E}$

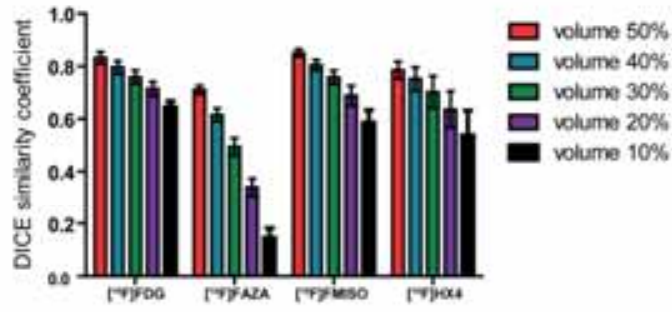

Figure 3.3 Voxel-to-voxel analysis and spatial reproducibility. A) Dynamic scans were used for voxel-tovoxel comparison between scans obtained at 2, 3, 4 or 5 hours p.i. and the 6 hour scan, shown in the representative scatter plot. B) Correlation coefficients of voxel-to-voxel analyses over a short-term time frame. For all tracers $n=4 \mathrm{C}$ ) Correlation coefficients of voxel-to-voxel analyses over a 48-hour time frame shows $\left[{ }^{18} \mathrm{~F}\right]$ FAZA that is significantly lower: $\mathrm{P}_{\mathrm{FDG}}$ vs. FAZA $=0.0061, \mathrm{P}_{\mathrm{FAZA} \text { vs. FMISO }}=0.0003, \mathrm{P}_{\mathrm{FAZA}}$ vs HX4 $=0.0121$. Each dot represents 1 animal, and the mean is indicated. D) Representative $\left[{ }^{18} \mathrm{~F}\right] \mathrm{HX} 4$ PET/CT image of a tumor crosssection visualized over a 48-hour time interval. Delineation on the test and retest scan shows the $30 \%$ of the total tumor volume with the highest SUV. Overlap fractions of these regions were calculated and represent the DICE similarity coefficient. E) Spatial reproducibility over a 48-hour time frame is presented per tracer as a percentage of total tumor volume. $\left[{ }^{18} \mathrm{~F}\right] \mathrm{FDG} n=4,\left[{ }^{18} \mathrm{~F}\right] \mathrm{FAZA} n=7,\left[{ }^{18} \mathrm{~F}\right] \mathrm{FMISO} n=8$ and $\left[{ }^{18} \mathrm{~F}\right] \mathrm{HX} 4 \mathrm{n}=4$. Data are the means \pm SEM.

$\left[{ }^{18} \mathrm{~F}\right] \mathrm{FMISO}(0.86)$ and $\left[{ }^{18} \mathrm{~F}\right] \mathrm{HX} 4(0.76)$; whereas $\left[{ }^{18} \mathrm{~F}\right] \mathrm{FAZA}$ had a significantly $(\mathrm{P}<0.05)$ lower correlation coefficient (0.52) (figure 3.3C). To further investigate spatial reproducibility, we calculated an overall DICE similarity coefficient in which the high uptake region as a percentage of the total tumor volume from the first scan was 

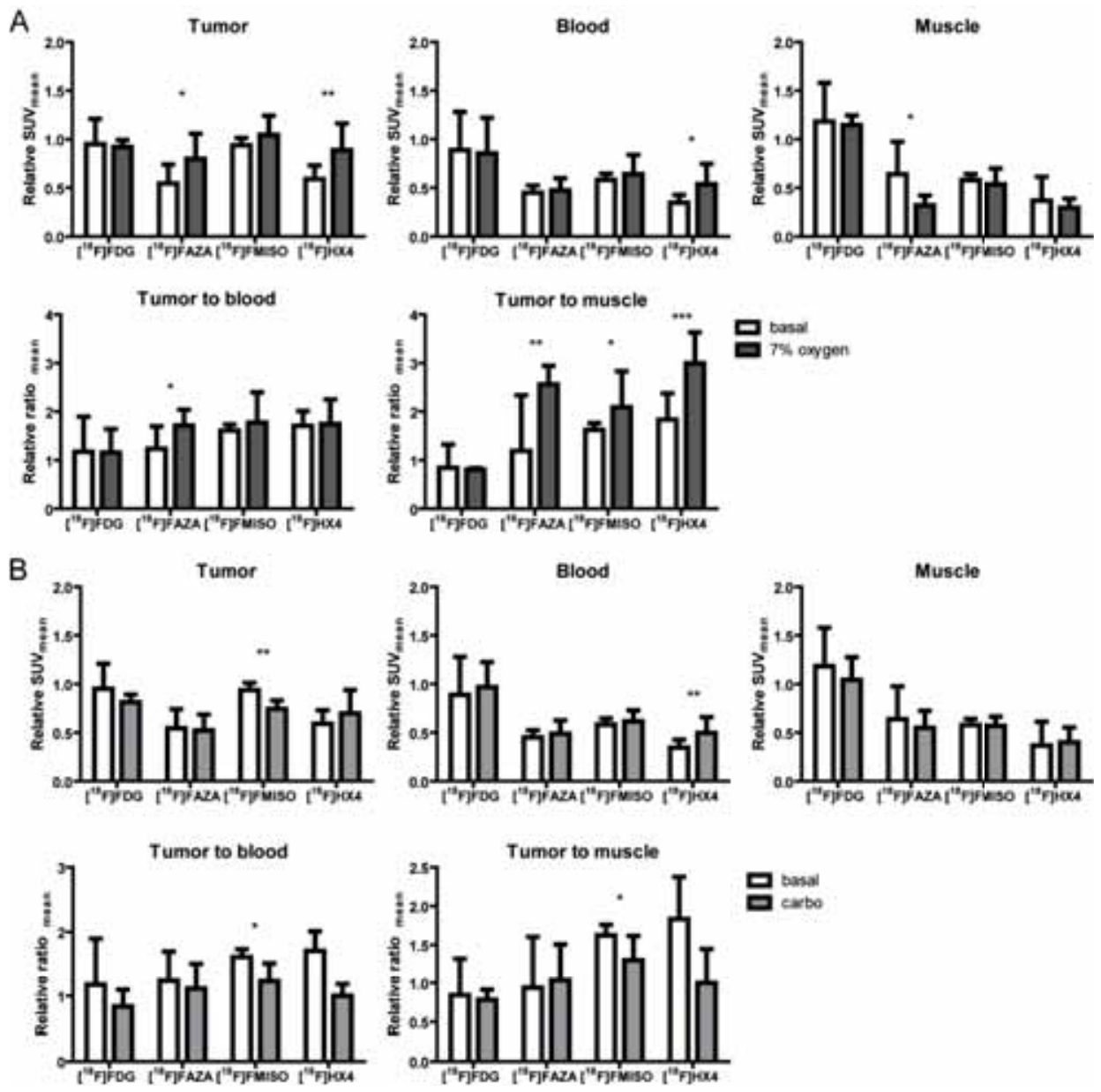

Figure 3.4 Oxygen modification. Relative $S U V_{\text {mean }}$ and tracer ratios compare untreated rats (basal) with those breathing 7\% oxgyen (7\% oxygen) A) and those receiving nicotinamide/carbogen (carbo) treatmetn B) . SUVs were calculated and compared 5 hours p.i. to 2 hours p.i. (vertical axis: relative SUV) for each organ separately and for the relative ratios TBR or TMR at 5 hours p.i. to 2 hours p.i. ${ }^{*} \mathrm{P}<0.05,{ }^{*}{ }^{*} \mathrm{P}<0.01$, *** $\mathrm{P}<0.001$. Data are means \pm SD for basal: $\left[{ }^{18} \mathrm{~F}\right] \mathrm{FDG} \mathrm{n}=12,\left[{ }^{18} \mathrm{~F}\right] \mathrm{FAZA} \mathrm{n}=14,\left[{ }^{18} \mathrm{~F}\right] \mathrm{FMISO} \mathrm{n}=16$ and $\left[{ }^{18} \mathrm{~F}\right] \mathrm{HX} 4$ $n=18$, for $7 \%$ oxygen: $\left[{ }^{18} F\right] F D G n=7,\left[{ }^{18} F\right] F A Z A n=7,\left[{ }^{18} F\right] F M I S O n=8$ and $\left[{ }^{18} F\right] H X 4 n=8$, for carbo: $\left[{ }^{18} F\right]$ FDG $n=8$, $\left[{ }^{18} \mathrm{~F}\right] \mathrm{FAZA} n=8,\left[{ }^{18} \mathrm{~F}\right] \mathrm{FMISO} n=6$ and $\left[{ }^{18} \mathrm{~F}\right] \mathrm{HX} 4 \mathrm{n}=14$. SUV $\max =$ maximum standard uptake value; TBR=tumor-toblood ratio; TMR tumor to muscle ratio.

compared to the same percentage of total volume area from a second scan (figure 3.3D). This analysis showed a high reproducibility for $\left[{ }^{18} \mathrm{~F}\right] \mathrm{FDG}(0.83),\left[{ }^{18} \mathrm{~F}\right] \mathrm{FMISO}(0.85)$, $\left[{ }^{18} \mathrm{~F}\right] \mathrm{HX} 4(0.79)$ and $\left[{ }^{18} \mathrm{~F}\right] \mathrm{FAZA}(0.71)$ in a comparison of the $50 \%$ tumor volume with the highest tracer uptake. However, when only the $10 \%$ highest uptake of the tumor volume was selected, $\left[{ }^{18} \mathrm{~F}\right] \mathrm{FDG},\left[{ }^{18} \mathrm{~F}\right] \mathrm{FMISO}$ and $\left[{ }^{18} \mathrm{~F}\right] \mathrm{HX} 4$ showed high spatial 
reproducibility $\left(0.65,0.59\right.$ and 0.49 , respectively), whereas $\left[{ }^{18} \mathrm{~F}\right] \mathrm{FAZA}$ showed a significant lower $(p<0.05)$ spatial reproducibility of 0.14 (figure $3.3 \mathrm{E})$.

Rats were exposed to 7\% oxygen breathing (figure 3.1B), mimicking acute hypoxia. The relative $S V_{\text {mean }}$ tracer uptake after $7 \%$ oxygen treatment was significantly increased for $\left[{ }^{18} \mathrm{~F}\right] \mathrm{HX} 4(\mathrm{P}<0.01)$ and $\left[{ }^{18} \mathrm{~F}\right] \mathrm{FAZA}(\mathrm{P}<0.05)$ in the tumor, compared to that in the untreated animals (figure $3.4 \mathrm{~A}$ ). The mean TBR for $\left[{ }^{18} \mathrm{~F}\right] \mathrm{FAZA}$ was significantly increased $(P<0.05)$; the mean TMR showed a significant increase for all 3 hypoxia tracers $\left(\left[{ }^{18} \mathrm{~F}\right]\right.$ FAZA $\mathrm{P}<0.01,\left[{ }^{18} \mathrm{~F}\right] \mathrm{FMISO} \mathrm{P}<0.05$ and $\left.\left[{ }^{18} \mathrm{~F}\right] \mathrm{HX} 4 \mathrm{P}<0.01\right)$ (figure $\left.3.4 \mathrm{~A}\right)$. When the effect of maximal tumor uptake on increased hypoxia was studied, no significant effects were observed, although there was a trend towards increased uptake of $\left[{ }^{18} \mathrm{~F}\right] \mathrm{HX} 4$ in the tumor (figure S3.2A).

The reversibility of tracer uptake on tumor reoxygenation was examined by treating the rats with nicotinamide and carbogen (figure 3.1B). Relative SUV mean $_{\text {(figure S3.1A) }}$ indicated that $\left[{ }^{18} \mathrm{~F}\right] \mathrm{FMISO}$ remained stable over time in the baseline situation, whereas for other tracers decreased. Influencing tumors towards a more oxygenated state only caused a decrease in $\left[{ }^{18} \mathrm{~F}\right] \mathrm{FMISO}$ tumor uptake and did not change uptake of $\left[{ }^{18} \mathrm{~F}\right] \mathrm{HX} 4$ and $\left[{ }^{18} \mathrm{~F}\right] \mathrm{FAZA}$ (figure 3.4B). Blood uptake values increased for $\left[{ }^{18} \mathrm{~F}\right] \mathrm{HX} 4$, but uptake in the muscle was not influenced. Mean TBR and TMR significantly decreased for $\left[{ }^{18} \mathrm{~F}\right] \mathrm{FMISO}$ and maximal values demonstrated comparable results (figure S3.2B).

\section{Discussion}

This study compares the frequently used hypoxia PET tracers $\left[{ }^{18} \mathrm{~F}\right] \mathrm{FMISO},\left[{ }^{18} \mathrm{~F}\right] \mathrm{FAZA}$, $\left[{ }^{18} \mathrm{~F}\right] \mathrm{HX} 4$ and the metabolic tracer $\left[{ }^{18} \mathrm{~F}\right] \mathrm{FDG}$ in an animal tumor model to assess their tumor-to-background ratios, spatial reproducibility and sensitivity to oxygen modification.

The rat rhabdomyosarcoma R1 model with a large hypoxic fraction (18) was chosen to ensure sufficient visualization of the uptake of hypoxia tracers using a preclinical model on a clinical PET/CT scanner. Although some variation in tumor volume was seen, the hypoxic fraction was shown to be stable within this tumor model (18). PET acquisition was performed from the time of injection untill 6 hours p.i. to determine the optimal uptake in the tumor and highest TBR. In this study, we found the most optimal TBR for $\left[{ }^{18} \mathrm{~F}\right] \mathrm{FAZA}$ at 2 hours p.i. This finding is consistent with clinical studies in head and neck squamous cell carcinoma and non-small cell lung cancer (NSCLC) 
where imaging at 4 hours p.i. did not improve the TBR compared to 2 hours p.i. (1921). $\left[{ }^{18} \mathrm{~F}\right] \mathrm{HX} 4$ shows an optimal TBR at 3 hours p.i which was also observed in an NSCLC patient study where image contrast did improve from 2 to 4 hours p.i. (22). As clinically demonstrated, $\left[{ }^{18} \mathrm{~F}\right] \mathrm{FMISO}$ does not show plateau formation, and has better TBR at later time points (23) which was also observed in this preclinical study. Comparative studies already have indicated that $\left[{ }^{18} \mathrm{~F}\right] \mathrm{HX} 4$ imaging in head and neck cancer patients at 1.5 hours p.i. was found to have TMR properties similar to those of $\left[{ }^{18} \mathrm{~F}\right] \mathrm{FMISO}$ at 2 hours p.i. (24). This is also reflected in the biological half-life of the tracers, which is much higher in normal tissue for $\left[{ }^{18} \mathrm{~F}\right.$ FMISO (clinical:12-13 hours (22), preclinical: 4.5 hours) than for $\left[{ }^{18} \mathrm{~F}\right] \mathrm{HX} 4$ (clinical: 4.3 hours (22), preclinical: 2.2 hours) or $\left[{ }^{18} \mathrm{~F}\right] \mathrm{FAZA}$ (preclinical: 2.8 hours). The findings from this preclinical study are in line with those from available clinical studies and although caution needs to be taken in extrapolation of the data, this might indicate that the results found here in this animal model can be translated to some extend to the clinical setting.

One disparity between clinical and preclinical studies is the use of anesthetic drugs. In this study pentobarbital was used, and although it was shown that this causes a reduction in the radioactivity in blood and muscle, it did not influence tracer uptake in the tumor, nor did it lead to a significant change in tumor-to-background ratios (25).

The ultimate goal of tumor hypoxia imaging is to improve treatment outcome either by detecting hypoxia to aid in the decision to add specific anti-hypoxia drugs or by adapting radiation therapy using image guidance. Considering that hypoxia imaging can be used to generate personalized intensity modulated radiation therapy plans in which these radiation-resistant parts of the tumor can be boosted $(26,27)$, it would be desirable to have a tracer that shows stable uptake over time so that a single scan could be used for several days of treatment. Voxel-to-voxel analyses resulted in high reproducibility for all tracers within a 6-hour scan. Examining spatial reproducibility by comparing a high uptake region revealed good overlap between 2 consecutive scans 48h apart for $\left[{ }^{18} \mathrm{~F}\right] \mathrm{FMISO},\left[{ }^{18} \mathrm{~F}\right] \mathrm{HX} 4$ and $\left[{ }^{18} \mathrm{~F}\right] \mathrm{FDG}$. For $\left[{ }^{18} \mathrm{~F}\right] \mathrm{FMISO}$ this was also reported in a recent clinical head and neck patient study in which 2 scans were highly reproducible over 48 hours (28). However, voxel-to-voxel analysis of $\left[{ }^{18} \mathrm{~F}\right.$ ]FMISO uptake over a 3-day interval found a correlation of the hypoxic distribution in less than $50 \%$ of the head and neck cancer patients (29). [ ${ }^{18} \mathrm{~F}$ FMISO in the same patient population and during chemoradiotherapy showed a stable conformation of the hypoxic subvolumes (30). Our data show that reproducibility of $\left[{ }^{18} \mathrm{~F}\right] \mathrm{FAZA}$ is poor after 48 hours, even without additional anticancer treatment. This is surprising given the fact that all 
investigated hypoxia tracers are based on the same nitroimidazole trapping mechanism. Contradictions in $\left[{ }^{18}\right.$ F]FAZA reproducibility are observed between different preclinical and clinical studies, which might also be caused by the differences in metabolism among organisms. Preclinical micro-PET analysis on $\left[{ }^{18} \mathrm{~F}\right] \mathrm{FAZA}$ uptake showed voxel-to-voxel reproducibility between 2 baseline scans 24 hours apart; even after fractionated radiation therapy, a fairly stable intratumoral tracer distribution was observed (31). However, in a clinical trial, $\left[{ }^{18} \mathrm{~F}\right]$ FAZA uptake was evaluated after several rounds of radiation therapy treatment and hypoxic regions were found not to be in the same location (19). Although $\left[{ }^{18} \mathrm{~F}\right] \mathrm{HX} 4$ shows good reproducibility in first clinical experiments (22), the stability of $\left[{ }^{18} \mathrm{~F}\right] \mathrm{HX} 4$ in detecting the hypoxic fraction during therapy needs to be further assessed. Uptake of $\left[{ }^{18} \mathrm{~F}\right] \mathrm{FDG}$ was clearly distinguishable from background and was highly reproducible, demonstrating the outstanding application of $\left[{ }^{18}\right.$ F]FDG in the detection of tumors. However, we consider $\left[{ }^{18}\right.$ F]FDG to be a metabolic tracer rather that a marker for hypoxia.

Because tumor hypoxia is a dynamic process that consists of both chronic and acute hypoxia, a tumor's oxygen status changes continuously, and most hypoxia tracers mainly detect the chronic hypoxic fraction. However, there are suggestions that acute hypoxia also plays a prominent role in determining the treatment outcome (32). Changing a tumor's oxygen status by clamping or reduced oxygen breathing mimics this dynamic process and gives the opportunity to study the behavior of tracer uptake under these conditions. In the ideal situation, one would wish a hypoxia tracer to rapidly and specifically accumulate in the hypoxic regions with fast clearance in the nonhypoxic tissues. Binding of the tracer would be irreversible, and no circulating free available tracer would be present.

Previous studies have shown that a treatment combining nicotinamide and carbogen increases a tumor's oxygen status $(9,33)$, whereas $7 \%$ oxygen breathing increases the hypoxic fraction (9). In this study, the oxygen modification was applied only 2.5 hours after tracer injection. Increasing the hypoxic fraction during tracer accumulation is dependent on the presence of unbound, circulating tracer. For all hypoxia tracers, circulating tracer was present after 3 hours, based on the measured activity in the blood (SUV of 2.4 for $\left[{ }^{18} \mathrm{~F}\right] F D G ; 0.3$ for $\left[{ }^{18} \mathrm{~F}\right] F A Z A ; 0.8$ for $\left[{ }^{18} \mathrm{~F}\right] \mathrm{FMISO}$, and 0.3 for $\left.\left[{ }^{18} \mathrm{~F}\right] \mathrm{HX} 4\right)$ however only $\left[{ }^{18} \mathrm{~F}\right] \mathrm{FAZA}$ and $\left[{ }^{18} \mathrm{~F}\right] \mathrm{HX} 4$ showed increased uptake in the tumor after $7 \%$ oxygen breathing. This effect was observed mainly in the mean values rather than in the maximum values, indicating that the tumor's overall oxygenation was altered, whereas the maximum value is determined by the severe hypoxic regions that 
will be less affected by this treatment. Exposure to high oxygen concentrations at 2.5 hours after tracer injection would prevent further accumulation or reverse tracer binding. For $\left[{ }^{18} \mathrm{~F}\right] \mathrm{FAZA}$, preclinical data are available that show reduced uptake after pure oxygen or carbogen breathing in tumor bearing mice $(8,34)$. In our experimental setting only $\left[{ }^{18} \mathrm{~F}\right] \mathrm{FMISO}$ showed a lower uptake upon reduced hypoxia. Together with the results of constant accumulation of $\left[{ }^{18} \mathrm{~F}\right] \mathrm{FMISO}$ in the tumor over time these data suggest that further accumulation is prevented when reducing the hypoxic fraction. Previous studies observed that $\left[{ }^{18} \mathrm{~F}\right] \mathrm{FMISO}$ uptake in squamous cell carcinoma bearing mice was influenced by the altered breathing condition (35). These experiments challenged the tracers to their limits and tried to mimic the changing oxygen concentrations in a tumor. It must be kept in mind that these results are influenced by tumor and animal model chosen and that the tracer metabolism is different in patients. Furthermore, exposing animals to modified oxygen concentration will introduce changes to the whole organism that might influence the distribution and metabolism of the tracer. Our data suggest that $\left[{ }^{18} \mathrm{~F}\right] \mathrm{HX} 4$ and $\left[{ }^{18} \mathrm{~F}\right] \mathrm{FAZA}$ are more sensitive to acute hypoxia while $\left[{ }^{18} \mathrm{~F}\right] \mathrm{FMISO}$ uptake is influenced by reoxygenation.

\section{Conclusions}

In conclusion, all investigated tracers showed different characteristics. The ultimate hypoxia tracer has not been developed, but this and other studies show that hypoxia imaging using the existing tracers gives extra information that can be very useful in the treatment of cancer patients.

\section{Acknowledgements}

We acknowledge financial support from the Center for Translational Molecular Medicine framework 030-103 (AIR FORCE), European Union seventh framework programmes (METOXIA grant agreement no: 222741 and ARTFORCE grant agreement no: 257144) and the Kankeronderzoekfonds Limburg from the Health Foundation Limburg.

\section{Conflicts of interest}

The authors declare that they have no conflict of interest. 


\section{Supplementary Data}

\section{Materials \& Methods}

Images were acquired using a clinical PET/CT scanner (Siemens Biograph 40, Siemens Healthcare) with an axial field of view (FoV) of $162 \mathrm{~mm}$, a transaxial FoV of $605 \mathrm{~mm}$ and a spatial resolution of $5.3 \mathrm{~mm}$ FWHM at the center of the FoV. The PET data were attenuation corrected using the acquired CT images. Also, correction for scatter (3D), randoms, dead time and decay of injected radionuclides was applied. First a topogram was acquired followed by a whole body CT scan using a $1 \mathrm{~mm}$ reconstructed slice thickness and a pitch of 0.8. For PET imaging an emission scan in list mode (LM) in one bed position was started simultaneously with tracer injection. LM data were rebinned using Fourier rebinning, and PET images were reconstructed for dynamic 60 minute imaging as $8 \times 15 \mathrm{sec}, 4 \times 30 \mathrm{sec}, 2 \times 1$ minute, $2 \times 2$ minutes and $10 \times 5$ minutes. The reconstructed voxel size was $3.04 \mathrm{~mm}$ in all dimensions. Every hour a similar scan was performed till 6 hours p.i. using a 20 minute emission scan, reconstructed as $4 \times 5$ minutes.

Reconstructed PET/CT images were analyzed in dedicated software (TrueD VC60; Siemens). Manual delineation of the volume of interest (VOI) was performed on the combined PET/CT image to obtain activity values $(\mathrm{Bq} / \mathrm{ml})$ for the tumor, muscle of the hind leg and blood pool (heart). After correcting the activity data for decay, data were quantified by calculating the SUV (activity in VOI / (injected dose/weight of animal)). SUV $_{\text {max }}$ indicates the averaged maximum SUV inside the VOI, SUV $V_{\text {mean }}$ is the averaged mean activity in the VOI. Tumor-to-blood ratios (TBR) and tumor-to-muscle (TMR) ratios were calculated (SUV tumor / SUV blood or muscle, respectively) using either mean or maximal values for both VOIs.

Non-rigid registration of the tumor was performed using in house developed registration software (36). The tumor contours were non-rigidly registered using an intensity based registration method (Morphons, 10 iterations, 8 resolution scales). A weighted sum accumulation of the deformation field was performed with a Gaussian regularization filter of 1.5 times the voxel size. The resulting deformation field was applied to the CT and the PET image of the rat. Next, the $50 \%$ of the total tumor volume with the highest SUV was defined, and the overlapping volume was calculated between the two scans. This was repeated on 40,30, 20 and $10 \%$ of the total tumor volume. 


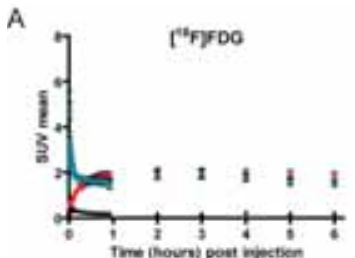

THFMISO

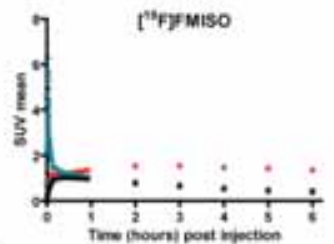

B

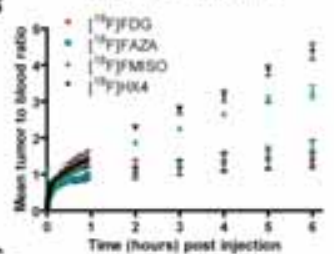

C
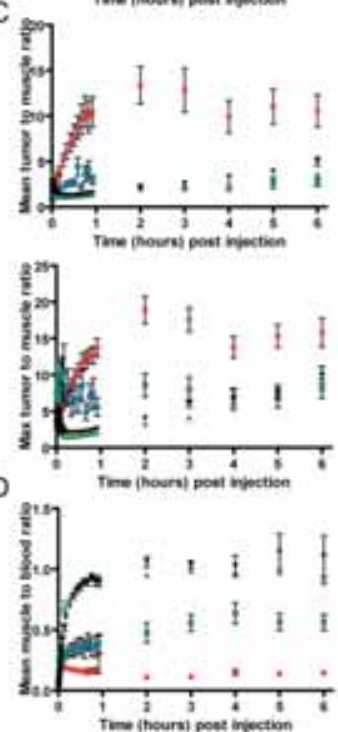

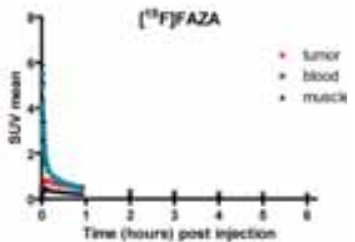

$\mathrm{I}^{\mathrm{H} F p o x}$

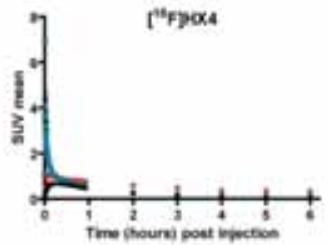

3 noi
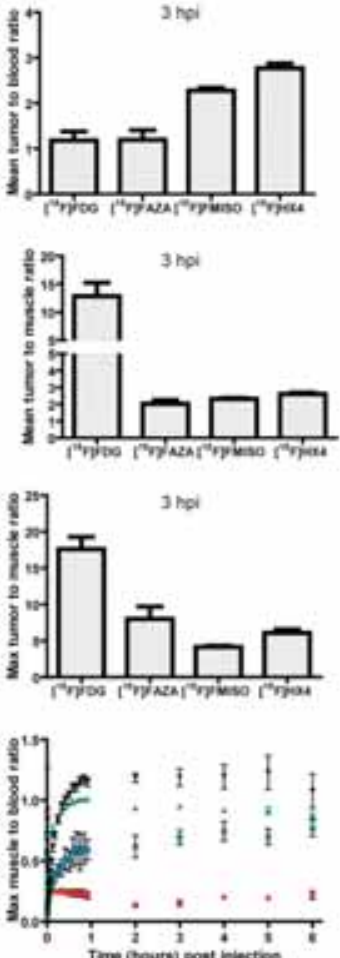

Supplement figure 3.1 Mean SUV and tumor-to-background ratios. a) SUV $V_{\text {mean }}$ of the tumor (red), blood (blue) and muscle (black) over time. $\left[{ }^{18} \mathrm{~F}\right] \mathrm{FDG} n=12,\left[{ }^{18} \mathrm{~F}\right] \mathrm{FAZA} n=13,\left[{ }^{18} \mathrm{~F}\right] \mathrm{FMISO} n=16$ and $\left[{ }^{18} \mathrm{~F}\right] \mathrm{HX} 4 \mathrm{n}=18 \mathrm{~b}$ ) Mean TBR over time for $\left[{ }^{18} \mathrm{~F}\right] \mathrm{FDG}$ (red), $\left[{ }^{18} \mathrm{~F}\right] F A Z A$ (blue), $\left[{ }^{18} \mathrm{~F}\right] \mathrm{FMISO}$ (green) and $\left[{ }^{18} \mathrm{~F}\right] \mathrm{HX} 4$ (black) with TBR tracer comparison at three hours post-injection. $\left[{ }^{18} \mathrm{~F}\right] \mathrm{HX} 4$ had significant better TBR compared with the other tracers $\left[{ }^{18} \mathrm{~F}\right] F D G \mathrm{n}=12,\left[{ }^{18} \mathrm{~F}\right] \mathrm{FAZA} \mathrm{n}=13,\left[{ }^{18} \mathrm{~F}\right] \mathrm{FMISO} \mathrm{n}=16$ and $\left[{ }^{18} \mathrm{~F}\right] \mathrm{HX} 4 \mathrm{n}=18 \mathrm{c}$ ) Mean tumor-to-muscle ratio over time. On the right side the mean TMR comparison of the four tracers at 3 hours p.i.: Mean TMR for $\left[{ }^{18} \mathrm{~F}\right] \mathrm{HX} 4$ was significantly higher than for the other hypoxia tracers: ${ }^{*} \mathrm{P}_{\mathrm{FAZA}}$ vs $\mathrm{HX4}=0.0114$ and ${ }^{*} \mathrm{P}_{\mathrm{FM}}{ }_{\mathrm{FIsO}}$ vs ${ }_{\mathrm{HX}}=0.0102\left[{ }^{18} \mathrm{~F}\right] \mathrm{FDG} \mathrm{n}=12,\left[{ }^{18} \mathrm{~F}\right] \mathrm{FAZA} \mathrm{n}=14,\left[{ }^{18} \mathrm{~F}\right] \mathrm{FMISO} \mathrm{n}=16$ and $\left.\left[{ }^{18} \mathrm{~F}\right] \mathrm{HX} 4 \mathrm{n}=18 . \mathrm{d}\right)$ Mean and maximal muscleto-blood ratio (MBR) over time. ${ }^{*} \mathrm{P}<0.05$. $\left[{ }^{18} \mathrm{~F}\right] \mathrm{FDG} \mathrm{n}=12,\left[{ }^{18} \mathrm{~F}\right] \mathrm{FAZA} \mathrm{n}=14,\left[{ }^{18} \mathrm{~F}\right] \mathrm{FMISO} \mathrm{n}=16$ and $\left[{ }^{18} \mathrm{~F}\right] \mathrm{HX} 4$ $\mathrm{n}=18$. Data are means \pm SEM. 
A
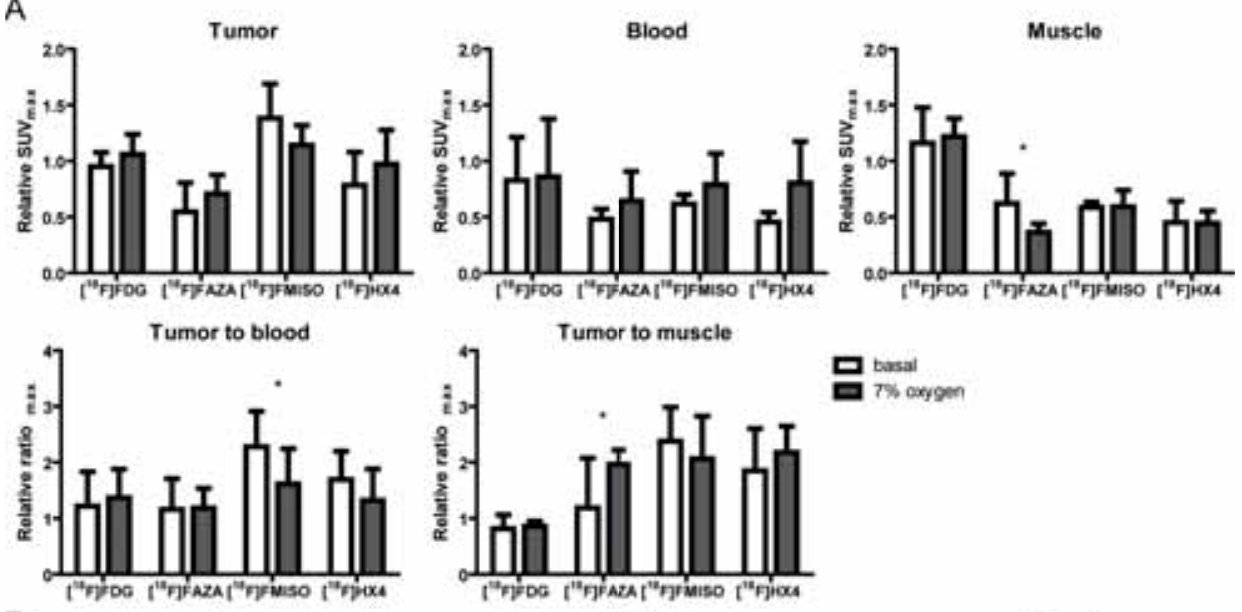

B
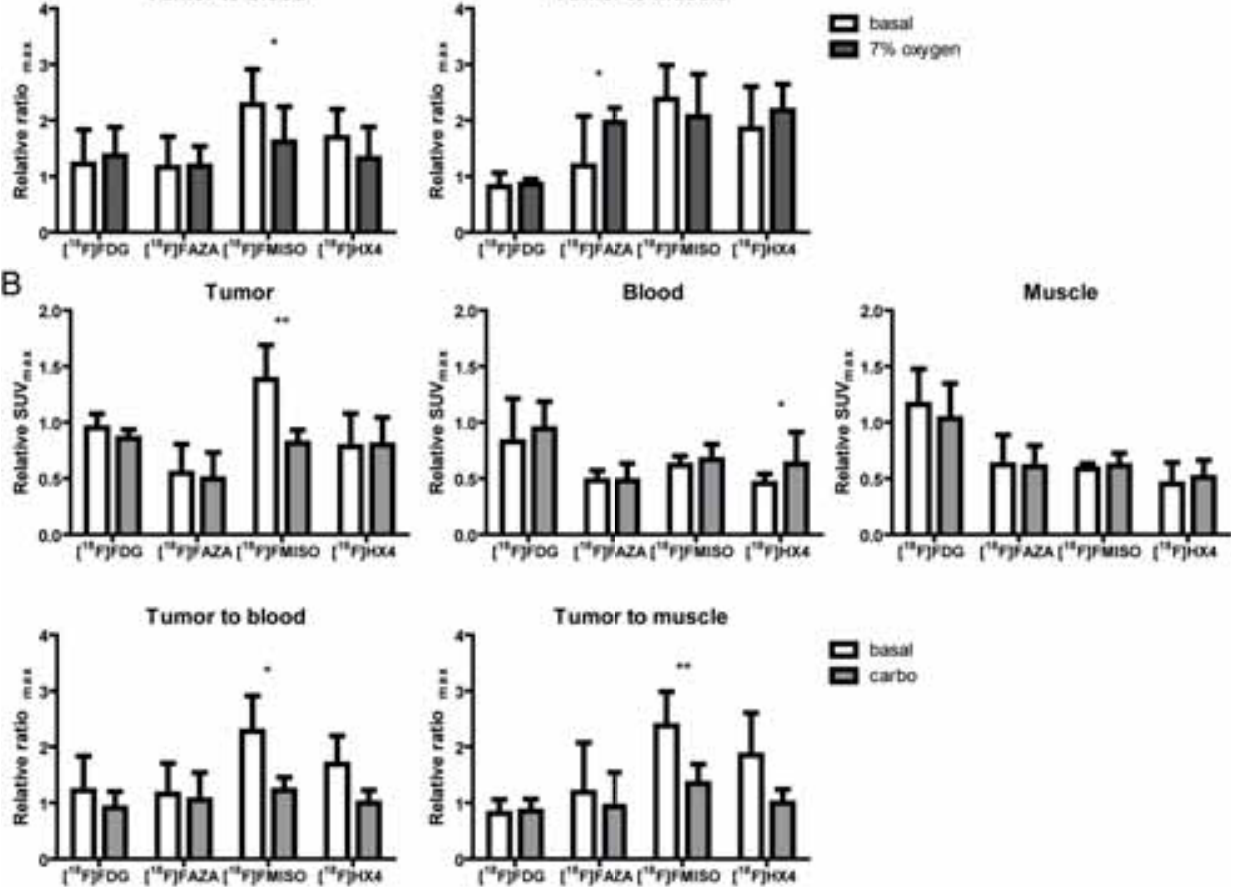

Supplement figure 3.2 Oxygen modification. Relative $S U V_{\max }$ or tracer ratios comparing untreated rats (basal) with a) 7\% oxygen breathing (7\% oxygen) or b) nicotinamide/carbogen (carbo) treated rats. SUV's are calculated and represented as 5 hours p.i. relative to 2 hours p.i. for each organ separately or for the calculated TBR or TMR. ${ }^{*} \mathrm{P}<0.05,{ }^{*} \mathrm{P}<0.01$. Data are means \pm SD for basal: $\left[{ }^{18} \mathrm{~F}\right] \mathrm{FDG} n=12,\left[{ }^{18} \mathrm{~F}\right] \mathrm{FAZA} n=14$, $\left[{ }^{18}\right.$ F]FMISO $n=16$ and $\left[{ }^{18} \mathrm{~F}\right] \mathrm{HX} 4 \mathrm{n}=18$, for $7 \%$ oxygen: $\left[{ }^{18} \mathrm{~F}\right] \mathrm{FDG} n=7,\left[{ }^{18} \mathrm{~F}\right] \mathrm{FAZA} n=7,\left[{ }^{18} \mathrm{~F}\right] \mathrm{FMISO} n=8$ and $\left[{ }^{18} \mathrm{~F}\right] \mathrm{HX} 4$ $n=8$, for carbo: $\left[{ }^{18} \mathrm{~F}\right]$ FDG $n=8,\left[{ }^{18} \mathrm{~F}\right] \mathrm{FAZA} n=8,\left[{ }^{18} \mathrm{~F}\right] \mathrm{FMISO} n=6$ and $\left[{ }^{18} \mathrm{~F}\right] \mathrm{HX} \mathrm{n}=14$ 


\section{References}

1. Hammond EM, Asselin MC, Forster D, O'Connor JP, Senra JM, Williams KJ. The meaning, measurement and modification of hypoxia in the laboratory and the clinic. Clin Oncol (R Coll Radiol). 2014;26:277-88.

2. Horsman MR, Mortensen LS, Petersen JB, Busk M, Overgaard J. Imaging hypoxia to improve radiotherapy outcome. Nat Rev Clin Oncol. 2012;9:674-87.

3. Brown JM, Giaccia AJ. The unique physiology of solid tumors: opportunities (and problems) for cancer therapy. Cancer Res. 1998;58:1408-16.

4. Krohn KA, Link JM, Mason RP. Molecular imaging of hypoxia. J Nucl Med. 2008;49 Suppl 2:129S-48S

5. Dubois L, Landuyt W, Cloetens L, Bol A, Bormans G, Haustermans K, et al. [18F]EF3 is not superior to [18F]FMISO for PET-based hypoxia evaluation as measured in a rat rhabdomyosarcoma tumour model. Eur J Nucl Med Mol Imaging. 2009;36:209-18.

6. Gronroos T, Bentzen L, Marjamaki P, Murata R, Horsman MR, Keiding S, et al. Comparison of the biodistribution of two hypoxia markers [18F]FETNIM and [18F]FMISO in an experimental mammary carcinoma. Eur J Nucl Med Mol Imaging. 2004;31:513-20.

7. Mahy P, De Bast M, de Groot T, Cheguillaume A, Gillart J, Haustermans K, et al. Comparative pharmacokinetics, biodistribution, metabolism and hypoxia-dependent uptake of [18F]-EF3 and [18F]MISO in rodent tumor models. Radiother Oncol. 2008;89:353-60.

8. Reischl G, Dorow DS, Cullinane C, Katsifis A, Roselt P, Binns D, et al. Imaging of tumor hypoxia with [124I]IAZA in comparison with [18F]FMISO and [18F]FAZA--first small animal PET results. J Pharm Pharm Sci. 2007;10:203-11.

9. Dubois LJ, Lieuwes NG, Janssen MH, Peeters WJ, Windhorst AD, Walsh JC, et al. Preclinical evaluation and validation of [18F]HX4, a promising hypoxia marker for PET imaging. Proc Natl Acad Sci U S A. 2011;108:14620-5.

10. Carlin S, Zhang H, Reese M, Ramos NN, Chen Q, Ricketts SA. A comparison of the imaging characteristics and microregional distribution of 4 hypoxia PET tracers. Journal of nuclear medicine : official publication, Society of Nuclear Medicine. 2014;55:515-21.

11. Eschmann SM, Paulsen F, Reimold M, Dittmann H, Welz S, Reischl G, et al. Prognostic impact of hypoxia imaging with 18F-misonidazole PET in non-small cell lung cancer and head and neck cancer before radiotherapy. J Nucl Med. 2005;46:253-60.

12. Mortensen LS, Johansen J, Kallehauge J, Primdahl H, Busk M, Lassen P, et al. FAZA PET/CT hypoxia imaging in patients with squamous cell carcinoma of the head and neck treated with radiotherapy: results from the DAHANCA 24 trial. Radiother Oncol. 2012;105:14-20.

13. van Loon J, Janssen $\mathrm{MH}$, Ollers $\mathrm{M}$, Aerts HJ, Dubois L, Hochstenbag $M$, et al. PET imaging of hypoxia using [18F]HX4: a phase I trial. Eur J Nucl Med Mol Imaging. 2010;37:1663-8.

14. Doss M, Zhang JJ, Belanger MJ, Stubbs JB, Hostetler ED, Alpaugh K, et al. Biodistribution and radiation dosimetry of the hypoxia marker 18F-HX4 in monkeys and humans determined by using whole-body PET/CT. Nucl Med Commun. 2010;31:1016-24.

15. Lim JL, Berridge MS. An efficient radiosynthesis of [18F]fluoromisonidazole. Appl Radiat Isot. 1993;44:1085-91.

16. Reischl G, Ehrlichmann W, Bieg C, Solbach C, Kumar P, Wiebe LI, et al. Preparation of the hypoxia imaging PET tracer [18F]FAZA: reaction parameters and automation. Appl Radiat Isot. 2005;62:897-901.

17. Walsh JC, Kolb HC. Applications of click chemistry in radiopharmaceutical development. Chimia (Aarau). 2010;64:29-33. 
18. Dubois L, Landuyt W, Haustermans K, Dupont P, Bormans G, Vermaelen P, et al. Evaluation of hypoxia in an experimental rat tumour model by [(18)F]fluoromisonidazole PET and immunohistochemistry. Br J Cancer. 2004;91:1947-54.

19. Servagi-Vernat S, Differding S, Hanin FX, Labar D, Bol A, Lee JA, et al. A prospective clinical study of FFAZA PET-CT hypoxia imaging in head and neck squamous cell carcinoma before and during radiation therapy. European journal of nuclear medicine and molecular imaging. 2014.

20. Souvatzoglou M, Grosu AL, Roper B, Krause BJ, Beck R, Reischl G, et al. Tumour hypoxia imaging with [18F]FAZA PET in head and neck cancer patients: a pilot study. European journal of nuclear medicine and molecular imaging. 2007;34:1566-75.

21. Trinkaus ME, Blum R, Rischin D, Callahan J, Bressel M, Segard T, et al. Imaging of hypoxia with 18F-FAZA PET in patients with locally advanced non-small cell lung cancer treated with definitive chemoradiotherapy. J Med Imaging Radiat Oncol. 2013;57:475-81.

22. Zegers $\mathrm{CM}$, van Elmpt W, Wierts R, Reymen B, Sharifi H, Ollers MC, et al. Hypoxia imaging with [(1)(8)F]HX4 PET in NSCLC patients: defining optimal imaging parameters. Radiotherapy and oncology : journal of the European Society for Therapeutic Radiology and Oncology. 2013;109:58-64.

23. Abolmaali N, Haase R, Koch A, Zips D, Steinbach J, Baumann M, et al. Two or four hour [(1)(8)F]FMISOPET in HNSCC. When is the contrast best? Nuklearmedizin. 2011;50:22-7.

24. Chen L, Zhang Z, Kolb HC, Walsh JC, Zhang J, Guan Y. (1)(8)F-HX4 hypoxia imaging with PET/CT in head and neck cancer: a comparison with (1)(8)F-FMISO. Nucl Med Commun. 2012;33:1096-102.

25. Christian N, Bol A, De Bast M, Labar D, Lee J, Mahy P, et al. Determination of tumour hypoxia with the PET tracer [18F]EF3: improvement of the tumour-to-background ratio in a mouse tumour model. Eur J Nucl Med Mol Imaging. 2007;34:1348-54.

26. Hendrickson K, Phillips M, Smith W, Peterson L, Krohn K, Rajendran J. Hypoxia imaging with [F-18] FMISO-PET in head and neck cancer: potential for guiding intensity modulated radiation therapy in overcoming hypoxia-induced treatment resistance. Radiother Oncol. 2011;101:369-75.

27. Thorwarth D, Eschmann SM, Paulsen F, Alber M. Hypoxia dose painting by numbers: a planning study. Int J Radiat Oncol Biol Phys. 2007;68:291-300.

28. Okamoto S, Shiga T, Yasuda K, Ito YM, Magota K, Kasai K, et al. High reproducibility of tumor hypoxia evaluated by 18F-fluoromisonidazole PET for head and neck cancer. J Nucl Med. 2013;54:201-7.

29. Nehmeh SA, Lee NY, Schroder H, Squire O, Zanzonico PB, Erdi YE, et al. Reproducibility of intratumor distribution of (18)F-fluoromisonidazole in head and neck cancer. Int J Radiat Oncol Biol Phys. 2008;70:235-42.

30. Bittner MI, Wiedenmann N, Bucher S, Hentschel M, Mix M, Weber WA, et al. Exploratory geographical analysis of hypoxic subvolumes using 18F-MISO-PET imaging in patients with head and neck cancer in the course of primary chemoradiotherapy. Radiother Oncol. 2013;108:511-6.

31. Busk M, Mortensen LS, Nordsmark M, Overgaard J, Jakobsen S, Hansen KV, et al. PET hypoxia imaging with FAZA: reproducibility at baseline and during fractionated radiotherapy in tumour-bearing mice. Eur J Nucl Med Mol Imaging. 2013;40:186-97.

32. Brown M. Henry S. Kaplan Distinguished Scientist Award Lecture 2007. The remarkable yin and yang of tumour hypoxia. Int J Radiat Biol. 2010;86:907-17.

33. Horsman MR, Overgaard J. Preclinical studies on how to deal with patient intolerance to nicotinamide and carbogen. Radiother Oncol. 2004;70:301-9.

34. Piert M, Machulla HJ, Picchio M, Reischl G, Ziegler S, Kumar P, et al. Hypoxia-specific tumor imaging with 18F-fluoroazomycin arabinoside. J Nucl Med. 2005;46:106-13.

35. Matsumoto K, Szajek L, Krishna MC, Cook JA, Seidel J, Grimes K, et al. The influence of tumor oxygenation on hypoxia imaging in murine squamous cell carcinoma using [64Cu]Cu-ATSM or [18F]Fluoromisonidazole positron emission tomography. Int J Oncol. 2007;30:873-81. 
36. Janssens G, de Xivry JO, Fekkes S, Dekker A, Macq B, Lambin P, et al. Evaluation of nonrigid registration models for interfraction dose accumulation in radiotherapy. Med Phys. 2009;36:4268-76. 



\section{Chapter 4}

\section{$\left[{ }^{18} \mathrm{~F}\right] \mathrm{VM} 4-037$ microPET imaging and biodistribution of two in vivo CAIX- expressing tumor models}

In Press: Mol Imaging Biol.

Sarah G.J.A. Peeters, Ludwig Dubois, Natasja G. Lieuwes, Dennis Laan, Martien Mooijer, Robert C. Schuit, Daniela Vullo, Claudiu T. Supuran, Jonas Eriksson, Albert D. Windhorst, Philippe Lambin 


\section{Abstract}

Purpose $\left[{ }^{18} \mathrm{~F}\right] \mathrm{VM} 4-037$ was recently developed as a positron emission tomography (PET) tracer for the detection of carbonic anhydrase IX (CAIX), a tumor-specific protein upregulated under hypoxic conditions. In this study, the accumulation of [ $\left.{ }^{18} \mathrm{~F}\right] \mathrm{VM} 4-037$ was determined in two CAIX-expressing preclinical human tumor models.

Procedures U373 and HT29 tumor-bearing animals were injected with [ $\left.{ }^{18} \mathrm{~F}\right] \mathrm{VM} 4-037$ and underwent microPET imaging up to $4 \mathrm{~h}$ post-injection (h p.i.). Biodistribution throughout the different organs was assessed at 2 and $4 \mathrm{~h}$ p.i. using gamma counting. Results MicroPET imaging showed high [ $\left.{ }^{18} \mathrm{~F}\right] \mathrm{VM} 4-037$ uptake in the abdominal region, and biodistribution revealed high radioactivity in the kidney, ileum, colon, liver, stomach, and bladder. Although high CAIX expression was confirmed in both tumor models, tumor uptake assessed with microPET and biodistribution experiments was comparable to background tissues.

Conclusions In this study, $\left[{ }^{18} \mathrm{~F}\right] \mathrm{VM} 4-037$ does not specifically accumulate in CAIXexpressing tumors, indicating that the tracer is not suitable for the detection of CAIX. 


\section{Introduction}

Carbonic anhydrase IX (CAIX) is a tumor-specific protein upregulated in response to hypoxia (1). Low oxygen concentrations are a common phenomenon in solid tumors (2). One of the key adaptations to this condition is the stabilization of 'hypoxiainducible factor' (HIF) (3), which regulates the transcription of several genes including the CA9 gene (1). CAIX plays a main role in the regulation of the cellular $\mathrm{pH}$. By catalyzing the reaction of $\mathrm{CO}_{2}$ and $\mathrm{H}_{2} \mathrm{O}$ to bicarbonate and a proton, CAIX preserves the stable intracellular environment and acidifies the extracellular environment (4). Furthermore, the expression of CAIX has been found to be a negative predictor for disease-free survival (5). Inhibition of CAIX function by targeted drugs would therefore be a promising anticancer approach.

Several specific CAIX inhibitors have been developed and described (6). Recent publications show that inhibition of CAIX results in reduced tumor growth in vivo, especially in combination with radiotherapy $(7,8)$. Furthermore, in vitro and in vivo results indicate the potential involvement of CAIX in processes like metastasis and invasion (9-11). Before clinical implementation of these specific inhibitors, it would be extremely valuable to have a screening method to assess the CAIX status of tumors. This would allow patient selection before the start of treatment. A new promising imaging radiopharmaceutical, $\left[{ }^{18} \mathrm{~F}\right] \mathrm{VM} 4-037$, has recently been proposed. So far, this radiopharmaceutical has been tested in healthy human volunteers, demonstrating the safety of $\left[{ }^{18} \mathrm{~F}\right] \mathrm{VM} 4-037(12)$. We assessed the tumor-specific uptake of this positron emission tomography (PET) radiopharmaceutical in two preclinical CAIX-expressing tumor models using microPET and biodistribution.

\section{Material and Methods}

\section{Synthesis of $\left[{ }^{18} \mathrm{~F}\right] \mathrm{VM}$ 4-037}

Synthesis of radioactive-labeled (S)-3-(4-(2-[ $\left.{ }^{18} \mathrm{~F}\right]$ fluoroethoxy)phenyl)-2-(3-methyl-2-(4((2-sulfamoylbenzo[d]thiazol-6-yloxy)methyl)-1H-1,2,3-triazol-1-yl)butanamido) propanoic acid, also referred to as $\left[{ }^{18} \mathrm{~F}\right] \mathrm{VM} 4-037$ was performed as described previously(12). The high-performance liquid chromatography (HPLC)-isolated $\left[{ }^{18}\right.$ F]VM4-037 fractions demonstrated a radiochemical purity always $>99 \%$. Specific activity was typically in the range of $15-50 \mathrm{GBq} / \mu \mathrm{mol}$ and the decay-corrected synthesis yield was $7-9 \%$. 


\section{Animal and tumor model, PET acquisition, and metabolism}

All animal experiments were approved by the ethical committee of the local universities. All applicable institutional and national guidelines for the care and use of animals were followed. U373 glioma (ATCC HTB-17) or HT29 (ATCC HTB-38) colorectal adenocarcinoma cells, resuspended in Basement Membrane Matrix (Matrigel ${ }^{\mathrm{TM}} \mathrm{BD}$ Biosciences), were subcutaneously injected into the lateral flank or the shoulder of NMRI (nu/nu) mice. Tumor volume was measured on regular basis using a Vernier caliper and calculated by the formula $A \times B \times C \times \pi / 6$ in which $A, B$ and $C$ are the three orthogonal diameters of the tumor, each corrected for the thickness of the skin. The minimum tumor volume for inclusion was $>300 \mathrm{~mm}^{3}$ to ensure clear visualization on the microPET. Upon an average tumor volume of $597 \mathrm{~mm}^{3}$ (range 358 - 981) and 1192 $\mathrm{mm}^{3}$ (range $\left.463-1745\right)$ for U373 $(n=4)$ and HT29 $(n=3)$ respectively, PET acquisition was performed under isoflurane anesthesia (induction $4 \%$, maintenance $1-2 \%$ ).

PET imaging was performed using the Focus 120 microPET (Siemens Medical Solutions USA, Inc) with an axial field of view (FOV) of $7.6 \mathrm{~cm}$, a transaxial FOV of $10 \mathrm{~cm}$ and a resolution of approximately $1.5 \mathrm{~mm}$. The animals were positioned in the center of the FOV of the microPET using a laser-positioning system.

A bolus injection of $\left[{ }^{18} \mathrm{~F}\right] \mathrm{VM} 4-037(3.79 \pm 0.55 \mathrm{MBq})$ was given via a catheter placed in the lateral tail vein. PET acquisitions for the U373 tumor model ( $n=4$ mice, all bearing a tumor) were performed at time points 0,120 and 240 minutes post-injection (p.i.) using a 15-minute emission scan. Image acquirement of the HT29 tumor model ( $n=4$ mice, three of which had a tumor) was performed at 30, 60, 90 and 120 minutes after injection using a 10-min emission scan. Each scan was corrected for random counts, dead time, and decay. List mode acquisition and reconstruction were performed as previously described (13). Images were visualized and analyzed using ASIPro VM software (version 6.3.3.0).

\section{Biodistribution}

After the final PET acquisition, animals were sacrificed and prepared for biodistribution. The tumor, blood, skin, bladder, heart, lungs, liver, spleen, stomach, ileum, colon, kidney, lymph nodes, salivary gland, muscle, and brain were weighed, and the amount of radioactivity in each organ was assessed using a gamma-well counter (1480 Wallac Wizard 3" automatic $\gamma$-counter; PerkinElmer, Inc.). Tumors were halved, after which one piece was used for gamma counting and the other half was 
snap frozen for protein isolation. Disintegrations were counted for $1 \mathrm{~min}$ for each sample using an energy window of 350 to $650 \mathrm{keV}$. Samples were corrected for background and decay, and the percentage of the injected dose per gram (\%ID/g) was calculated.

\section{Metabolite Analysis}

C57BL/6 mice were injected with $\left[{ }^{18} \mathrm{~F}\right] \mathrm{VM} 4-037(21.27 \pm 0.42 \mathrm{MBq})$. At $15 \min (\mathrm{n}=3)$ and 45 min $(n=3)$, animals were sacrificed and blood was collected via a heart puncture, centrifuged ( $4 \mathrm{~min}, 4000 \mathrm{rpm}$ ) and plasma was harvested and diluted with acidified water. An activated (10 $\mathrm{ml}$ of ethanol followed by $10 \mathrm{~mL}$ of water) Waters tC18 Sep-Pak solid phase extraction cartridge was used to separate the polar and apolar fractions, which were further analyzed with HPLC (Dionex UltiMate 3000), using a Phenomenex Gemini $5 \mu, 250 \times 10$ column as stationary phase. The mobile phase consisted of acetontirile (A) and a 90 to $15 \%$ gradient of $10 \mathrm{mM}$ phosphoric acid (B) at a flow of $3 \mathrm{ml} / \mathrm{min}^{-1}$.

\section{Western blotting}

Tumors were minced and protein was isolated using RIPA buffer completed with a protease inhibitor cocktail (complete EDTA-free; Roche, Indianapolis, USA). Protein concentrations were determined by Bradford assay (BioRad, Veenendaal, Netherlnds) and protein was separated on a $10 \%$ SDS-PAGE gel. After blotting onto a nitrocellulose membrane (GE Healthcare, Amersham Corp, UK) by electrotransfer, membranes were blocked in $5 \%$ non-fat dry milk and probed overnight with antibodies against CAIX (M75, kindly provided by S. Pastorekova, Institute of Virology, Slovak Academy of Science, Bratislava, Slovak Republic) and $\beta$-actin (Cell Signaling). Secondary antibodies ( $\alpha$-mouse IgG, Cell Signaling) were detected with Western blot detection reagents (Thermo Scientific Pierce, Rockford, USA).

\section{Delta pH assay}

HT29 cells were seeded and incubated for $24 \mathrm{~h}$ under hypoxic conditions $\left(0.2 \% \mathrm{O}_{2}, 5 \%\right.$ $\mathrm{CO}_{2}$, residual $\mathrm{N}_{2}$; MACS VA500 microaerophilic workstation, Don Whitley Scientific, Shipley, UK) with the addition of either vehicle (1 \% DMSO), VM4-037 (1 mM), or 4-(2aminoethyl)benzene sulfonamide (AEBS; $1 \mathrm{mM}$ ), a positive control for CAIX inhibition $(n=4)(4)$. The $\mathrm{pH}$ of the culture medium was immediately measured at the end of each experiment, as described in a previous study (14). The inhibitory constant (Ki) were obtained with a stopped-flow assay method (15). 
A

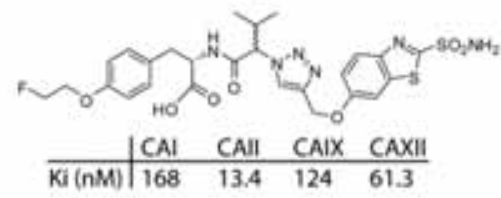

C

D
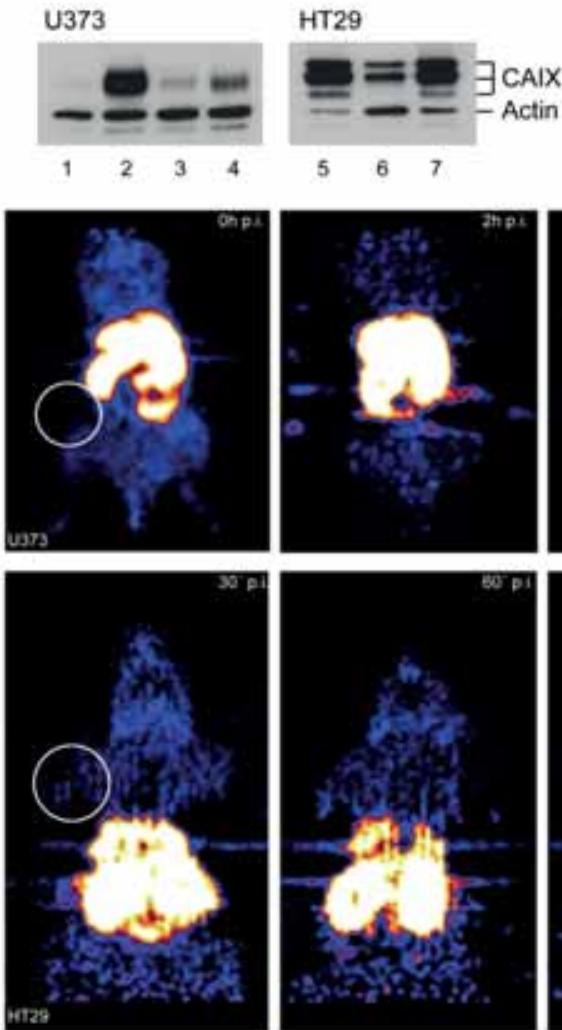

E

U373
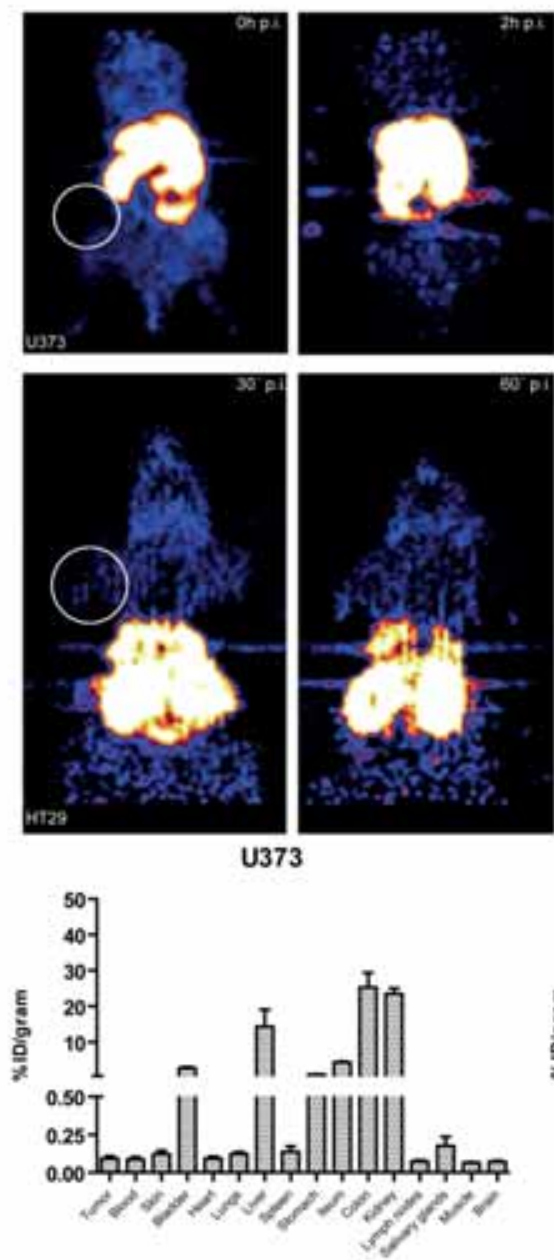

4 hours post injection
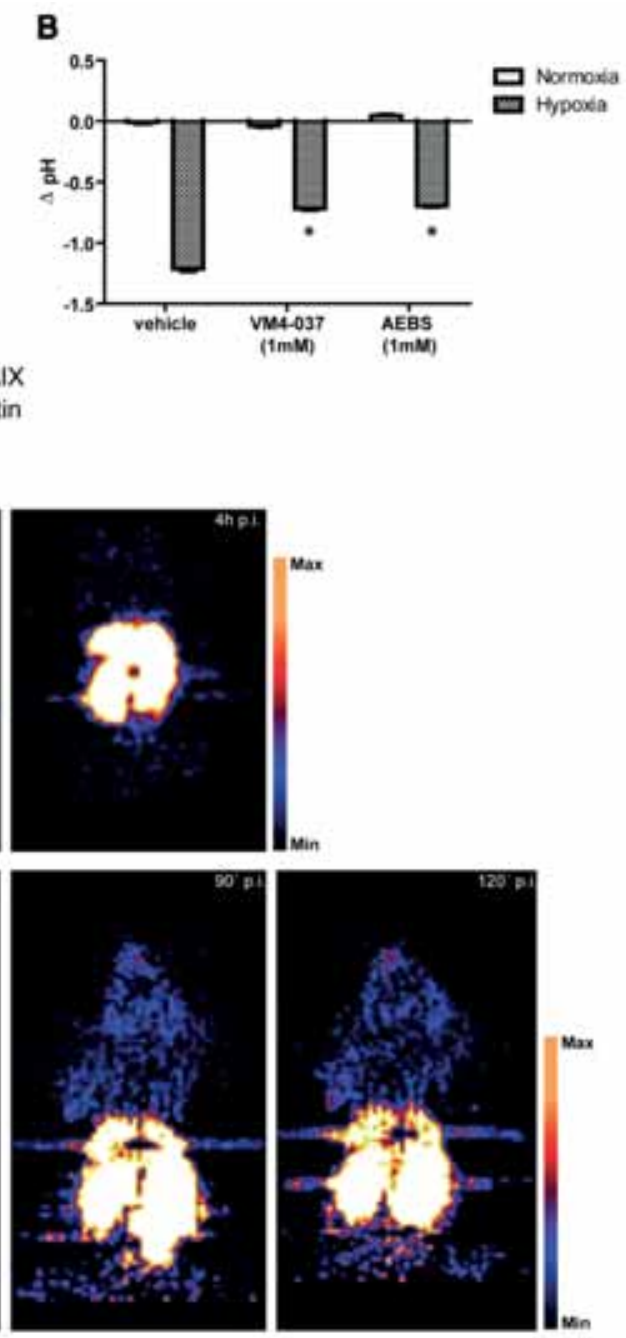

Hा 29

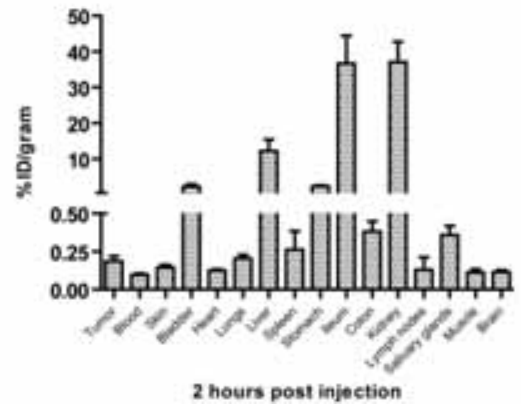


Figure 4.1 [ $\left.{ }^{18} \mathrm{~F}\right] \mathrm{VM}$ 4-037 in CAIX expressing tumors. A) Structure formula of VM4-037 with Ki values for CAI, CAIl, CAIX and CAXII B) In vitro delta pH assay on HT29 cells exposed for $24 \mathrm{~h}$ to $0.2 \%$ hypoxia either with vehicle, VM4-037, or the positive control AEBS $(n=4)$. Bars indicate mean plus SEM. C) Western blot analysis of U373 (number 1-4) or HT29 (number 5-7) protein samples from tumors excised from mice. The bands of CAIX and actin are indicated. D) microPET images of mice bearing U373 (upper, corresponding to figure. 4.1B mouse 1) or HT29 (lower, corresponding to figure 4.1B mouse 7) tumors at multiple time points. High uptake in yellow/white, low uptake in blue as represented by the color scale bars, expressed as Bq/cc. White circle indicates location of tumor. E) Biodistribution at 4 and 2 h p.i. from U373 $(n=4)$ or HT29 $(n=4$ mice, three of which had a tumor) tumor-bearing mice, respectively. Bars indicate mean plus SEM.

\section{Results}

The inhibitory constant of VM4-037 was determined for different carbonic anhydrases and showed a Ki of 124 nM for CAIX while for CAl this was 168 nM, CAll 13.4 nM and for CAXII $61.3 \mathrm{nM}$ (figure 4.1A). A delta $\mathrm{pH}$ assay was performed to verify the CAIXinhibiting potential of VM4-037. Upon hypoxic conditions, the extracellular environment acidifies, and this acidification is reduced upon incubation with either VM4-037 or the positive control AEBS (figure 4.1b). To assess CAIX expression of the in vivo tumors, protein levels were evaluated by Western blot. Although there was variation, all tumors expressed a certain level of CAIX (figure 4.1c).

PET acquisition was performed to investigate the distribution of $\left[{ }^{18} \mathrm{~F}\right] \mathrm{VM} 4-037$ throughout the animal's body and to detect specific uptake in the tumor (figure 4.1D). At the first time point, the shape of the mouse is clearly visible, and high uptake is seen in the abdominal region. Over time, the overall signal intensity decreased, except for the abdominal region. No signal was detected from the tumor region in any of the scans. In the HT29 model, the shape of the animal was also clearly visible, and high signals were detected in the abdominal region. However, no accumulation was observed in the tumor (figure 4.1D).

A biodistribution study performed on the U373 tumor-bearing animals at $4 \mathrm{~h}$ p.i. showed that the organs with more than $1 \%$ ID/g $\left[{ }^{18} \mathrm{~F}\right] \mathrm{VM} 4-037$ uptake were the colon, kidney, and liver, followed by the ileum, bladder, and stomach (figure 4.1E). Disintegrations detected from the tumor were not higher compared to background tissues like the muscle, blood, and skin. Assessing the biodistribution of $\left[{ }^{18} \mathrm{~F}\right] \mathrm{VM} 4-037$ at $2 \mathrm{~h} \mathrm{p.i.} \mathrm{in} \mathrm{the} \mathrm{HT29} \mathrm{tumor-bearing} \mathrm{animals} \mathrm{showed} \mathrm{an} \mathrm{uptake} \mathrm{of} \mathrm{above} 30 \% \mathrm{ID} / \mathrm{g}$ in the kidneys, ileum, and tissues and over $1 \% \mathrm{ID} / \mathrm{g}$ in the liver, bladder, and stomach 
(figure 4.1E). The percent injected dose per gram of the tumor was in the same range as in the low-uptake organs.

Metabolite analysis of $\left[{ }^{18} \mathrm{~F}\right] \mathrm{VM} 4-037$ revealed $87.8 \pm 1.5 \%$ and $43.1 \pm 3.0 \%$ of the tracer to be un-metabolized at 15 and $45 \mathrm{~min}$ p.i, respectively. Polar metabolites accounted for $11.1 \pm 2.0 \%$. Two apolar metabolites were found in the 45 -min samples, accounting for $27.2 \pm 1.9$ and $17.1 \pm 3.6 \%$.

\section{Discussion}

This study investigated the distribution of $\left[{ }^{18} \mathrm{~F}\right] \mathrm{VM} 4-037$ in tumor-bearing animals with the aim to determine the specific accumulation in CAIX-expressing tumors. We assessed the uptake of this tracer in two different CAIX-expressing tumor models using microPET and biodistribution.

Doss et al. assessed the distribution of $\left[{ }^{18} \mathrm{~F}\right] \mathrm{VM} 4-037$ in healthy volunteers (12), demonstrating the safety of this CAIX PET imaging tracer. Consistent with their results, both our microPET imaging and biodistribution data show a high uptake of the tracer in the kidneys and liver at both 2 and $4 \mathrm{~h}$ p.i. Doss et al. also found that radiation dosimetry showed high activity in the bladder at $4.8 \mathrm{~h}$ p.i. based on voiding models (12). In mice, this high-uptake organ was detected at $2 \mathrm{~h}$ p.i. together with the colon, ileum, and stomach. The difference in the pharmacokinetics of the tracer in humans compared to mice could be explained by a more rapid metabolism and clearance in mice. This may indicate that at later time points, $\left[{ }^{18} \mathrm{~F}\right] \mathrm{VM} 4-037$ might non-specifically accumulate in other human organs of the abdominal region besides the kidney and liver.

The main goal of our study was to assess the specific uptake of $\left[{ }^{18} \mathrm{~F}\right] \mathrm{VM} 4-037$ in CAIXexpressing tumors. Although the U373 tumors expressed CAIX, as validated by Western blot, microPET imaging could not distinguish tumor tissue from background tissue. The observed spread in CAIX expression in figure $4.1 \mathrm{~B}$ is part of the natural variation between the different tumors. A second tumor model with an even higher and more stable CAIX expression was used and was located on the shoulder of the animal preventing the abdominal scatter from interfering with the signal from the tumor. Furthermore, imaging was acquired more frequently in the first $2 \mathrm{~h}$ p.i. to ensure coverage of all relevant time points. However, at none of the time points was the tumor distinguishable from the background, suggesting that the tracer does not 
specifically accumulate in the CAIX-expressing tumors. Biodistribution results were used to clearly distinguish the uptake per organ, especially in the high-uptake regions and the tumor. This confirmed that the uptake of $\left[{ }^{18} \mathrm{~F}\right] \mathrm{VM} 4-037$ in the tumor was comparable with the non-specific, overall distribution throughout the body of the animals.

Recently, the uptake of $\left[{ }^{18} \mathrm{~F}\right] \mathrm{VM} 4-037$ was evaluated in kidney cancer patients. Modest uptake signal was detected in the tumors, but no correlation was found with staining for CAIX and CAXII. The authors suggest that larger studies are needed, since in this study only 11 patients underwent imaging with $\left[{ }^{18} \mathrm{~F}\right] \mathrm{VM} 4-037$ (16). Although CAIX is a potential marker for clear cell renal cell carcinoma (17), it is remarkable that uptake from the kidney tumors could be distinguished since the current study, as well as the study conducted by Doss et al., showed high non-tumor-related uptake of $\left[{ }^{18}\right.$ F]VM4037 in this organ.

A new CAIX targeting tracer $\left[{ }^{18} \mathrm{~F}\right] 4 \mathrm{~d}$ has recently shown to be clearly visible in HT-29 tumors (18). Furthermore, another CAIX targeting tracer $(\mathrm{HE})_{3}$-ZCAIX:1, based on Affibody molecules, has recently been developed and tested in CAIX-expressing SK-RC52 renal carcinoma xenografts. The advantage of this new class of molecules is their small size resulting in favorable characteristics regarding extravasation, tissue penetration, and clearance of unbound tracer. These studies indicate that there is a general interest in finding a specific marker to detect CAIX noninvasively (19).

The question remains why no specific tracer accumulation is observed in the tumor. There could be multiple reasons for the discrepancy between the promising in vitro results of VM4-037 and the lack of a signal from the tumor in vivo. The radiopharmaceutical was $>99 \%$ pure at the moment of injection, which means the radiopharmaceutical could not be a reason for the unexpected low tumor uptake. In vitro, the CAIX-expressing cells are easy accessible; in vivo, however, the bioavailability is influenced by the whole organism. Metabolite analysis showed that $43 \%$ of the tracer was still available in the blood plasma 45 min p.i., which should be sufficient for distribution throughout the body and detection of CAIX at least on the early time points investigated in this study. This indicates that metabolic degradation could not be the cause for the low CAIX specific accumulation. However, characteristics of the tumor microenvironment, that are absent in vitro, could influence the uptake of the tracer in the in vivo situation. 


\section{Conclusion}

Although $\left[{ }^{18} \mathrm{~F}\right.$ VMM4-037 demonstrates some activity against CAIX in the in vitro delta $\mathrm{pH}$ assay and in vivo metabolite analysis are favorable, both our in vivo microPET and biodistribution results show that the tracer does not specifically accumulate in CAIXexpressing tumors.

\section{Acknowledgements:}

The authors thank Marissa Rongen and Esther Kooijman for their assistance with performing the metabolite analysis. The authors acknowledge financial support from the CTMM framework (AIRFORCE project, grant 030-103), EU 6th Framework Program (Euroxy project ref. 2003-502932), the EU 7th Framework Program (Metoxia project ref. 2008-222741), the Kankeronderzoekfonds Limburg of the Health Foundation Limburg, and the Dutch Cancer Society (KWF UM 2012-5394, KWF MAC 2013-6089). 


\section{References}

1. Wykoff CC, Beasley NJ, Watson PH, Turner KJ, Pastorek J, Sibtain A, et al. Hypoxia-inducible expression of tumor-associated carbonic anhydrases. Cancer Res. 2000;60:7075-83.

2. Wilson WR, Hay MP. Targeting hypoxia in cancer therapy. Nat Rev Cancer. 2011;11:393-410.

3. Lendahl U, Lee KL, Yang $H$, Poellinger L. Generating specificity and diversity in the transcriptional response to hypoxia. Nat Rev Genet. 2009;10:821-32.

4. Svastova E, Hulikova A, Rafajova M, Zat'ovicova M, Gibadulinova A, Casini A, et al. Hypoxia activates the capacity of tumor-associated carbonic anhydrase IX to acidify extracellular $\mathrm{pH}$. FEBS Lett. 2004;577:439-45.

5. Generali D, Berruti A, Brizzi MP, Campo L, Bonardi S, Wigfield S, et al. Hypoxia-inducible factor-1alpha expression predicts a poor response to primary chemoendocrine therapy and disease-free survival in primary human breast cancer. Clin Cancer Res. 2006;12:4562-8.

6. McDonald PC, Winum JY, Supuran CT, Dedhar S. Recent developments in targeting carbonic anhydrase IX for cancer therapeutics. Oncotarget. 2012;3:84-97.

7. Dubois L, Peeters SG, van Kuijk SJ, Yaromina A, Lieuwes NG, Saraya R, et al. Targeting carbonic anhydrase IX by nitroimidazole based sulfamides enhances the therapeutic effect of tumor irradiation: a new concept of dual targeting drugs. Radiother Oncol. 2013;108:523-8.

8. Dubois L, Peeters S, Lieuwes NG, Geusens N, Thiry A, Wigfield S, et al. Specific inhibition of carbonic anhydrase IX activity enhances the in vivo therapeutic effect of tumor irradiation. Radiother Oncol. 2011;99:424-31.

9. Pacchiano F, Carta F, McDonald PC, Lou Y, Vullo D, Scozzafava A, et al. Ureido-substituted benzenesulfonamides potently inhibit carbonic anhydrase IX and show antimetastatic activity in a model of breast cancer metastasis. J Med Chem. 2011;54:1896-902.

10. Svastova E, Witarski W, Csaderova L, Kosik I, Skvarkova L, Hulikova A, et al. Carbonic anhydrase IX interacts with bicarbonate transporters in lamellipodia and increases cell migration via its catalytic domain. J Biol Chem. 2012;287:3392-402.

11. Lou Y, McDonald PC, Oloumi A, Chia S, Ostlund C, Ahmadi A, et al. Targeting tumor hypoxia: suppression of breast tumor growth and metastasis by novel carbonic anhydrase IX inhibitors. Cancer Res. 2011;71:3364-76.

12. Doss M, Kolb HC, Walsh JC, Mocharla VP, Zhu Z, Haka M, et al. Biodistribution and radiation dosimetry of the carbonic anhydrase IX imaging agent [(18) F]VM4-037 determined from PET/CT scans in healthy volunteers. Mol Imaging Biol. 2014;16:739-46.

13. Aerts HJ, Dubois L, Perk L, Vermaelen P, van Dongen GA, Wouters BG, et al. Disparity between in vivo EGFR expression and 89Zr-labeled cetuximab uptake assessed with PET. J Nucl Med. 2009;50:123-31.

14. Dubois L, Douma K, Supuran CT, Chiu RK, van Zandvoort MA, Pastorekova S, et al. Imaging the hypoxia surrogate marker CA IX requires expression and catalytic activity for binding fluorescent sulfonamide inhibitors. Radiother Oncol. 2007;83:367-73.

15. Innocenti A, Vullo D, Scozzafava A, Supuran CT. Carbonic anhydrase inhibitors. Inhibition of isozymes I, II, IV, V, and IX with anions isosteric and isoelectronic with sulfate, nitrate, and carbonate. Bioorg Med Chem Lett. 2005;15:567-71.

16. Adam R. Metwalli, Baris Turkbey, Yolanda McKinney, Juanita Weaver, Nana Yaqub-Ogun, Maria Merino, et al. Results of a phase II trial of novel carbonic anhydrase IX radiotracer 18F-VM4-037 in renal cell carcinoma. 2014 Genitourinary Cancer Symposium. San Francisco; 2014. 
17. Zhao Z, Liao G, Li Y, Zhou S, Zou H, Fernando S. Prognostic value of carbonic anhydrase IX immunohistochemical expression in renal cell carcinoma: a meta-analysis of the literature. PLoS One. 2014;9:e114096.

18. Liu Z, Pourghiasian M, Radtke MA, Lau J, Pan J, Dias GM, et al. An organotrifluoroborate for broadly applicable one-step (18) f-labeling. Angewandte Chemie. 2014;53:11876-80.

19. Honarvar H, Garousi J, Gunneriusson E, Hoiden-Guthenberg I, Altai M, Widstrom C, et al. Imaging of CAIX-expressing xenografts in vivo using 99mTc-HEHEHE-ZCAIX:1 Affibody molecule. Int J Oncol. 2015;46:513-20. 


\section{Chapter 5}

\section{$\mathrm{TH}-302$ in combination with radiotherapy enhances the therapeutic outcome and is associated with pretreatment $\left[{ }^{18} \mathrm{~F}\right] \mathrm{HX} 4$ hypoxia PET imaging}

In Press: Clin Cancer Res

Sarah G.J.A. Peeters, Catharina M.L. Zegers, Rianne Biemans, Natasja G. Lieuwes, Ruud G.P.M. van Stiphout, Ala Yaromina, Jessica D. Sun, Charles P. Hart, Albert D. Windhorst, Wouter van Elmpt, Ludwig Dubois*, Philippe Lambin* 


\section{Statement of translational relevance}

Radiotherapy is applied to $50 \%$ of all cancer patients and is therefore an important cancer treatment modality. Hypoxia is a feature of solid tumors which gives the opportunity of a tumor-targeted approach. TH-302 is shown to be a promising hypoxiaactivated prodrug. Several clinical trials have already demonstrated the applicability of TH-302 as a monotherapy and in combination with chemotherapy. In this study, the efficacy of the combination treatment of $\mathrm{TH}-302$ and radiotherapy was assessed in two preclinical tumor models: the rat rhabdomyosarcoma model and the human non-small cell lung cancer $\mathrm{H} 460$ xenograft model. Furthermore, we monitored tumor hypoxia with an imaging biomarker used in clinical trials. Upon TH-302 treatment, the hypoxic cells, which are less sensitive to conventional anti-cancer therapies, were significantly decreased. We believe that the current study gives important directions for future clinical studies. 


\section{Abstract}

Purpose: Conventional anticancer treatments are often impaired by the presence of hypoxia. TH-302 selectively targets hypoxic tumor regions where it is converted into a cytotoxic agent. This study assessed the efficacy of the combination treatment of TH302 and radiotherapy in two preclinical tumor models. The effect of oxygen modification on the combination treatment was evaluated and the effect of $\mathrm{TH}-302$ on the hypoxic fraction (HF) was monitored using $\left[{ }^{18} \mathrm{~F}\right] \mathrm{HX} 4-\mathrm{PET}$ imaging and pimonidazole immunohistochemical stainings.

Material \& M ethods: Rhabdomyosarcoma R1 and H460 NSCLC tumor bearing animals were treated with $\mathrm{TH}-302$ and radiotherapy (8Gy, single dose). The tumor oxygenation status was altered by exposing animals to carbogen (95\% oxygen) and nicotinamide, $21 \%$ or $7 \%$ oxygen breathing during the course of the treatment. Tumor growth and treatment toxicity was monitored until the tumor reached 4 times its start volume (T4XSV).

Results: Both tumor models showed a growth delay after TH-302 treatment which further increased when combined with radiotherapy (enhancement ratio rhabdomyosarcoma 1.23; H460 1.49). TH-302 decreases the HF in both models, consistent with its hypoxia-targeting mechanism of action. Treatment efficacy was dependent on tumor oxygenation; increasing the tumor oxygen status abolished the effect of TH-302, while enhancing the HF enlarged TH-302's therapeutic effect. An association was observed in rhabdomyosarcoma tumors between the pre-treatment HF as measured by $\left[{ }^{18} \mathrm{~F}\right] \mathrm{HX} 4-\mathrm{PET}$ imaging and the T4xSV.

Conclusion: The combination of $\mathrm{TH}-302$ and radiotherapy is promising and warrants clinical testing preferably guided by the companion biomarker $\left[{ }^{18} \mathrm{~F}\right] \mathrm{HX} 4$ hypoxia PET imaging for patient selection. 


\section{Introduction}

Hypoxia is a common feature of solid tumors and is known to negatively influence treatment outcome $(1,2)$. Due to the disorganized vessel formation and consequently low oxygen concentrations, conventional chemotherapies and radiotherapies are less effective. To overcome hypoxia-induced treatment resistance, drugs have been developed that specifically target hypoxic tumor regions. These so-called 'hypoxia activated prodrugs' (HAP) are non-toxic under normal oxygen concentrations but are activated in environments with low oxygen concentrations. TH-302 is a second generation HAP of which the activation mechanism is based on the reduction of its 2nitroimidazole moiety. Only in the presence of certain reductases under low oxygen concentrations is the toxic effector bromo-isophosphoramide mustard (Br-IPM) selectively released and able to crosslink DNA leading to cell death.

Preclinical studies have assessed the therapeutic effect of $\mathrm{TH}-302$ alone or in combination with conventional chemotherapies in multiple xenograft models. TH-302 monotherapy led to reduced tumor growth in many of the xenograft models profiled, and was dependent on the hypoxic fraction (3). Combining TH-302 with several clinically used chemotherapeutics offers advantage over single agent treatment, although the treatment schedule and order of administration is of importance (4). A phase 1 study demonstrated the safety of TH-302 monotherapy in patients with solid malignancies (5). Other clinical phase I and II trials successfully combined the standard treatment doxorubicin with intravenous administration of $\mathrm{TH}-302$ in patients with advanced soft tissue sarcoma $(6,7)$ and standard treatment gemcitabine with $\mathrm{TH}-302$ in patients with advanced pancreatic cancer (8).

However, there are no published studies to assess the combination therapy of TH-302 and radiotherapy. Radiotherapy is one of the conventional treatment options applied to multiple cancer types and tumor hypoxia is a known radio-resistance factor. The combination of $\mathrm{TH}-302$ and radiotherapy is hypothesized to be complementary; where TH-302 specifically targets hypoxic cells, radiotherapy has the highest therapeutic gain in the well-oxygenated cells.

Positron emission tomography (PET) imaging is a noninvasive method characterizing the tumor oxygenation status in a 3-dimensional manner. Several PET tracers have been developed that specifically visualize hypoxic regions. One of those tracers, based on the same 2-nitroimidazole hypoxia sensing mechanism as TH-302, is $\left[{ }^{18} \mathrm{~F}\right] \mathrm{HX} 4$. Preclinical and clinical trials have shown that $\left[{ }^{18} \mathrm{~F}\right] \mathrm{HX} 4$ is a reliable tool for the non- 
invasive detection of hypoxic tumor regions (9-11). Since TH-302, like tirapazamine, is only expected to have a therapeutic effect when hypoxic regions are present in the tumor (12), [ $\left.{ }^{18} \mathrm{~F}\right] \mathrm{HX} 4$ PET imaging may function as a useful predictive biomarker.

In the current study we investigated the treatment effect of $\mathrm{TH}-302$ in combination with radiotherapy in two preclinical tumor models. We hypothesize that this combination therapy will enhance the therapeutic effect. Furthermore we investigate the causal relationship between TH-302 efficacy and the modified tumor oxygenation status which was assessed before and after treatment using $\left[{ }^{18} \mathrm{~F}\right] \mathrm{HX} 4$ hypoxia PET imaging and only after treatment with pimonidazole staining. We hypothesize that the pretreatment hypoxia $\left[{ }^{18} \mathrm{~F}\right] \mathrm{HX} 4 \mathrm{PET}$ imaging will correlate with the treatment outcome.

\section{Materials and Methods}

\section{Animal, tumor models and treatment schedules}

All animal experimental procedures were approved by the Animal Ethical Committee of Maastricht University and were in accordance with the Helsinki Declaration of 1975 as revised in 2000. All animals were monitored at least 3 times a week and their tumor volume was calculated using the formula: $A \times B \times C \times \pi / 6$ in which $A, B$ and $C$ are the three orthogonal diameters of the tumor as measured using a Vernier caliper, each corrected for the thickness of the skin. Animals were randomized into the different treatment groups (Supplementary figure 5.1A) and were monitored until 4 times start volume (T4xSV) was reached. For this calculation the start volume of the first day of TH-302 treatment was used and fitting of the data was based on the regrowth phase. TH-302 was supplied by Threshold Pharmaceuticals and dissolved in $0.9 \% \mathrm{NaCl}$ to a concentration of $5 \mathrm{mg} / \mathrm{ml}$.

\section{Experimental Models}

Syngeneic rhabdomyosarcoma R1 tumors $\left(1 \mathrm{~mm}^{3}\right)$ were implanted subcutaneously in the lateral flank of adult WAG/Rij rats. Experiments were started upon a mean tumor volume of $4.2 \mathrm{~cm}^{3}$ (range 2.0-8.1) to ensure a stable hypoxic fraction. Treatment was administered on 4 consecutive days (Supplementary figure 5.1A) and consisted of an intraperitoneal injection (i.p.; QDx4) with either $\mathrm{NaCl}$ or $\mathrm{TH}-302(25,50$ or $75 \mathrm{mg} / \mathrm{kg}$ ). Before the start of treatment a PET scan was made using $\left[{ }^{18} \mathrm{~F}\right] \mathrm{HX} 4$. Radiotherapy (RT; Varian Truebeam linear accelerator; $15 \mathrm{MeV}$ electrons) was applied in a single dose of 
0, 4, 8 or $12 \mathrm{~Gy}$ on day 3 of the treatment, 3 hours after $\mathrm{NaCl}$ or $\mathrm{TH}-302$ injection, 1 hour after oxygen modification. During both PET imaging and RT rats were anaesthetized using a mixture of ketamine/xylazine (i.p; 66.7 and $6.7 \mathrm{mg} / \mathrm{kg}$ respectively). During the 5 days of treatment ( 1 day PET imaging, 4 days of injections with TH-302 or vehicle), animals were exposed to modified oxygen concentrations for 4 hours per day in order to alter the hypoxic fraction of the tumor. The combination oxygen modification of nicotinamide (i.p. $500 \mathrm{mg} / \mathrm{kg}$ ) and carbogen ( $95 \%$ oxygen, $5 \%$ $\mathrm{CO}_{2} ; 5 \mathrm{~L} / \mathrm{min}$ ) consisted of a nicotinamide injection and 30 minutes later the exposure to carbogen breathing for 3.5 hours. In the middle of the nicotinamide/carbogen treatment $\mathrm{NaCl} / \mathrm{TH}-302$ was administered. Reduced oxygen breathing (7\%, residual $\mathrm{N}_{2}$; $2.5 \mathrm{~L} / \mathrm{min}$ ) was given for 4 hours with the $\mathrm{NaCl} / \mathrm{TH}-302$ injection after the first two hours. The injection of the $\left[{ }^{18} \mathrm{~F}\right] \mathrm{HX} 4$ PET tracer (mean 18.8 MBq, range 7.1-25.1 MBq; lateral tail vein using an intravenous line (Venoflux $0.4 \mathrm{~mm} \mathrm{G27)} \mathrm{flushed} \mathrm{with} 10 \%$ heparine) was given 2 hours before the end of the oxygen modification. PET imaging was performed 3 hours post tracer injection, as previously assessed (13). [ $\left.{ }^{18} \mathrm{~F}\right] \mathrm{HX} 4 \mathrm{PET}$ scans could not be performed on all treated animals due to production and supply difficulties. For the histological control animals, PET imaging was also performed on day 4 of the treatment.

H460 lung carcinoma cells were resuspended $\left(1 \times 10^{6}\right.$ cells $/ \mathrm{ml}$; ATCC HTB-177) in Basement Membrane Matrix (Matrigel ${ }^{\mathrm{TM}} \mathrm{BD}$ Biosciences) and injected in the lateral flank of NU-Foxn1-nu (NU/NU) mice. Experiments were started upon animals reaching a mean tumor volume of $225 \mathrm{~mm}^{3}$ (range $89-273 \mathrm{~mm}^{3}$ ). Mice were treated with either $\mathrm{NaCl}$ or $\mathrm{TH}-302$ (50 mg/kg) for 5 consecutive days (QDx5). Treatment was combined with RT in a single dose of 0 or $8 \mathrm{~Gy}$ on day 4 for which the animals were anaesthetized using a mixture of ketamine/xylazine (i.p; 66.7 and $6.7 \mathrm{mg} / \mathrm{kg}$ respectively). During the 5 days of treatment, animals were exposed to different oxygen concentrations; either a combination of nicotinamide $(500 \mathrm{mg} / \mathrm{kg}$ i.p.) and carbogen breathing (95\% oxygen, $5 \%$ CO2), $21 \%$ oxygen breathing (pressured air) or $7 \%$ oxygen breathing (residual $\mathrm{N}_{2}$ ). Total treatment time for all oxygen breathing schedules was 2.5 hours with the $\mathrm{NaCl} / \mathrm{TH}-302$ injection given 1 hour after the start of the treatment. Nicotinamide was administered 30 minutes before carbogen breathing was started, which was then applied for another 2 hours. RT was given within 1 hour after the oxygen treatment. Histological controls were administered with pimonidazole $160 \mathrm{mg} / \mathrm{kg}$, i.p.; Hypoxyprobe KIT, HP3-1000; Bio-connect) and Hoechst 33342 (15mg/kg, i.v.; SigmaAldrich) 1 hour and 1 minute before sacrificing respectively, followed by excision of the 
tumors which were snap-frozen in liquid nitrogen and stored at -80 degrees Celsius until being processed.

\section{PET Image acquisition and analysis}

Tracer synthesis of $\left[{ }^{18} \mathrm{~F}\right] \mathrm{HX} 4$ was performed as described previously (14). $\left[{ }^{18} \mathrm{~F}\right] \mathrm{HX} 4$ positron emission tomography (PET) images were acquired and analyzed using a clinical PET/CT scanner (Siemens Biograph 40, Siemens Healthcare) as previously described (13). A volume of interest in the heart (sphere with a radius of $3 \mathrm{~mm}$ ) was defined as background region. The threshold to define the hypoxic fraction (HF) was set at 4.5 times the background uptake, since this method results in a hypoxic fraction which is in agreement with the pimonidazole stainings based results of a previous study on the rat rhabdomyosarcoma model (15).

\section{Immunohistochemical staining and analysis}

Frozen $\mathrm{H} 460$ xenograft tumors were sectioned $(5 \mu \mathrm{m})$ and stained for hypoxia (pimonidazole), blood vessels (CD31) and perfusion (Hoechst 33342). Sections were fixed using cold acetone, rehydrated in tris-buffered saline with $0.2 \%$ Tween-20 (TBST) and preincubated with normal goat serum before exposing them to the primary antibody rabbit anti-pimonidazole (1:150; HP3-1000, Bio-connect) and rat anti-mouse CD31 (1:500; BD biosciences). After washing with TBST, incubation with the secondary antibody goat anti-rabbit Alexa594 (1:500) and goat anti-rat Alexa488 (1:750, both Invitrogen) was performed. Sections were mounted using fluorescent mounting medium (DakoCytomation) and scanned for pimonidazole, blood vessels and perfusion. After scanning, sections were stained for hematoxylin and eosin (H\&E).

Images were acquired using an Olympus BX51WI microscope equipped with a Hamamatsu EM-CCD C9100 digital camera, a motorized stage (Ludl Mac 2000) and a 10x objective. Micromanager 1.4 software was used for automated image acquisition (16). ImageJ version 1.49e (http://rsb.info.nih.gov/ij/) was used to stitch the images and perform quantitative analyses. All image recordings and analyses were performed by an investigator blinded to the subject coding. Viable tumor tissue was first delineated manually by excluding epidermis, stroma and necrotic tumor regions based on H\&E staining. The thresholds were set manually by two independent observers to discriminate signal from background. Finally the relative hypoxic fraction, microvessel density and perfusion were calculated by determining the positive fraction within the viable tumor area. 


\section{Statistics}

GraphPad Prism software (version 5.01 for Windows) was used to perform statistical analyses. To determine the statistical significance of differences between two independent groups of variables we used an unpaired t-test while for matched groups a paired t-test was applied. A 2-way ANOVA was performed in R v3.0.1 to determine synergistic effects. P-values $<0.05$ were considered to be significant.

\section{Results}

\section{Combination of TH-302 and radiotherapy}

The effect of TH-302 in combination with RT was assessed in two tumor models, a rhabdomyosarcoma rat syngeneic model and a $\mathrm{H} 460$ human non-small cell lung cancer (NSCLC) xenograft mouse model. The treatment dose of TH-302 for rhabdomyosarcoma was assessed in a 'tolerability' study, showing $25 \mathrm{mg} / \mathrm{kg}$ (QDx4) to be the most optimal dose without adverse effects (Supplementary figure 5.2). This dose was therefore selected for further experiments. The TH-302 treatment dose of $50 \mathrm{mg} / \mathrm{kg}$ (QDx5) for $\mathrm{H} 460$ was based on literature (3).

In both tumor models TH-302 treatment showed an inhibition of the tumor growth which was further reduced when $\mathrm{TH}-302$ administration was combined with a single dose of RT ( $8 \mathrm{~Gy}$; figure 5.1A). The time to reach 4 times start volume (T4xSV, figure $5.1 B$ ) was significantly delayed upon TH-302 monotherapy from $12.4 \pm 1.7$ (mean \pm SD) to $20.4 \pm 3.5$ days for rhabdomyosarcoma $(\mathrm{P}<0.001)$ and $7.1 \pm 2.4$ to $13.6 \pm 4.8$ days for $\mathrm{H} 460$ ( $\mathrm{P}=0.003)$. Compared to RT alone, T4xSV for the combination treatment was delayed from $24.9 \pm 3.0$ to $30.8 \pm 5.9$ for rhabdomyosarcoma $(P=0.026)$ and from 16.9 \pm 7.1 to $25.2 \pm 4.9$ for $\mathrm{H} 460$ ( $P=0.014$ ), resulting in an enhancement ratio (ER) of 1.23 and 1.49 respectively (figure 5.1B, Supplementary Table 5.1 and 5.2). In addition, the effect of TH-302 was also assessed in the rhabdomyosarcoma model in combination with 4 and $12 \mathrm{~Gy}$ of RT leading to an ER of 1.28 and 1.59 respectively (Supplementary figure 5.3A\&B, Supplementary Table 5.1). TH-302 has a radio-sensitizing effect in both tumor models and all RT doses. Moreover, the effect of the combination therapy TH302 and 12Gy RT in the rhabdomyosarcoma model was even synergistic. 
A

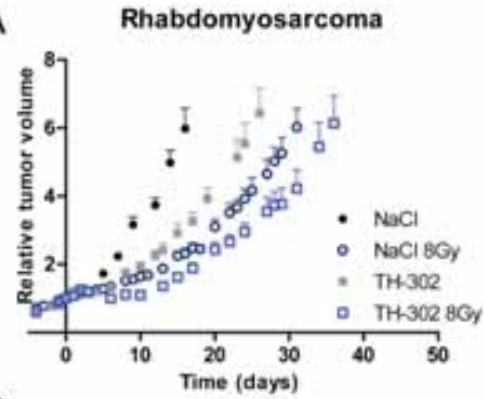

B
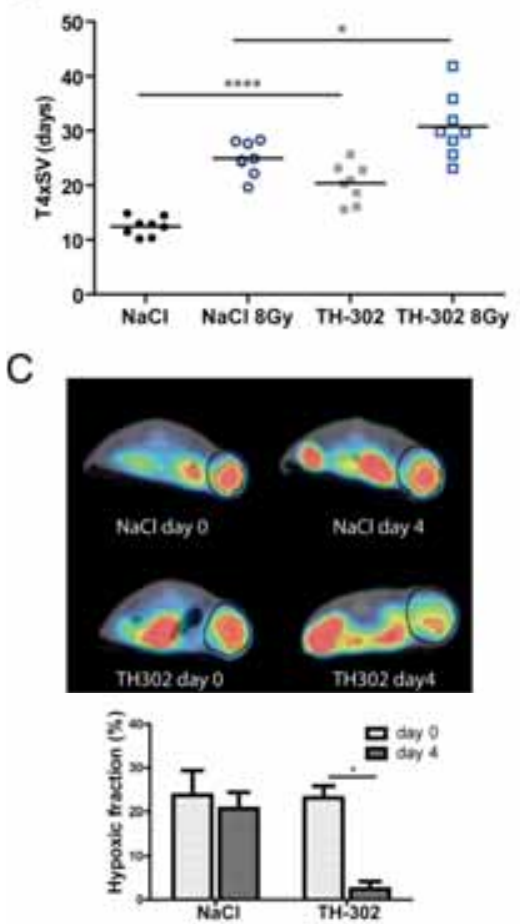

$\mathrm{H} 460$
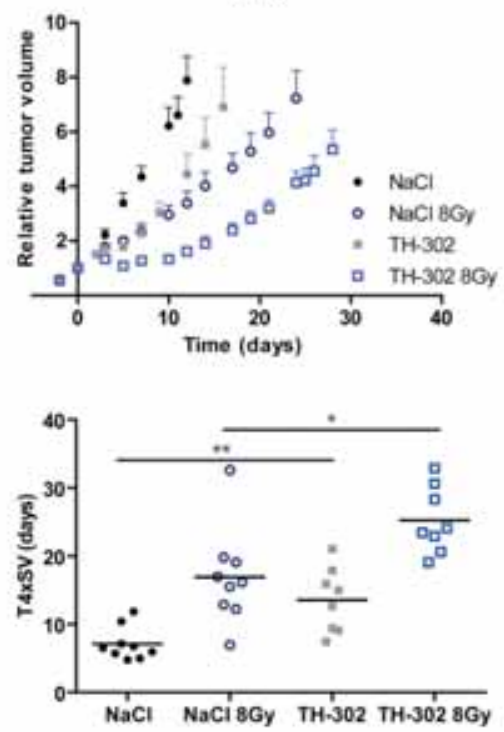

D
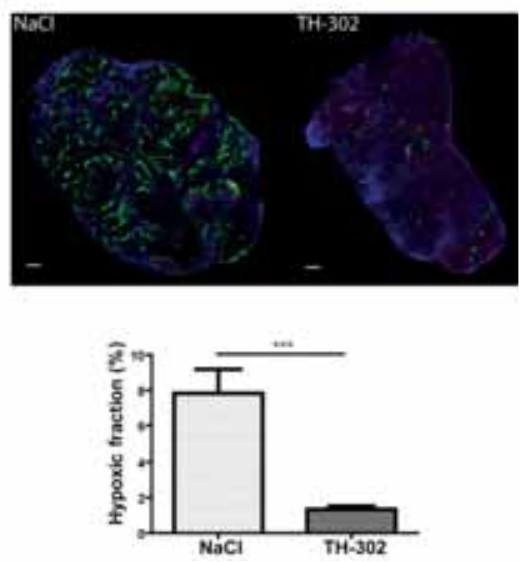

Figure 5.1 The combination of TH-302 and radiotherapy reduces tumor growth (RT; 8Gy) in a rhabdomyosarcoma $(n=8)$ and $H 460(n \geq 8)$ tumor model presented in A) growth curves and B) time to reach 4 times start volume (T4xSV). Animals were treated with either control $(\mathrm{NaCl})$ or $\mathrm{TH}-302$ administered to the rhabdomyosarcoma model for 4 consecutive days with a dose of $25 \mathrm{mg} / \mathrm{kg}$ and for the $\mathrm{H} 460$ model for 5 consecutive days using a $50 \mathrm{mg} / \mathrm{kg}$ dose. RT was applied on either the 3rd or 4th day of TH-302 treatment. The hypoxic fraction of the control animals and the animals treated with TH-302 were analyzed in $\mathrm{C}$ ) for rhabdomyosarcoma $(n=6)$ both before and immediately after treatment using $\left[{ }^{18} \mathrm{~F}\right] \mathrm{HX} 4$ hypoxia PET imaging (Top: representative $\left[{ }^{18} \mathrm{~F}\right] \mathrm{HX} 4 \mathrm{PET}$ images with the delineation of the total tumor volume in black and the HF in red. Bottom: quantification of HF per group) and in D) for $\mathrm{H} 460(\mathrm{n}=6)$ immediately after treatment using pimonidazole immunohistochemistry staining (Top: representative stainings, with hypoxia (pimonidazole) in green, perfusion (Hoechst) in blue and vessels (CD31) in red. The white scale bar indicates $500 \mu \mathrm{M}$. Bottom: quantification). Data represent average \pm SEM. ${ }^{*} \mathrm{P}<0.05, * * \mathrm{P}<0.005, * * * \mathrm{P}<0.001, * * * * \mathrm{P}<0.0001$ 
A

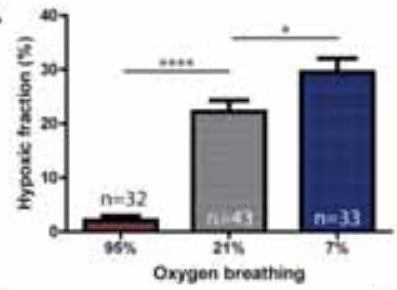

B
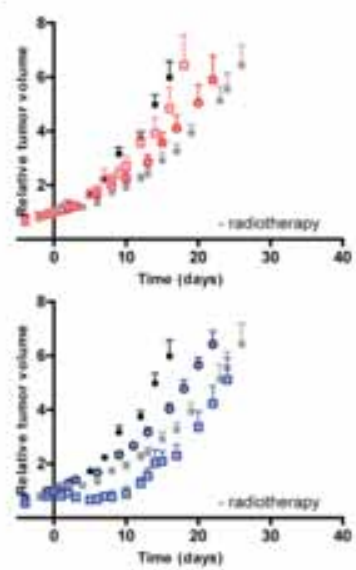

C
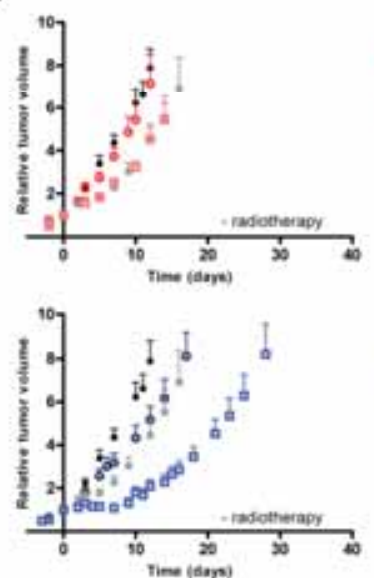

Rhabdomyosarcoma

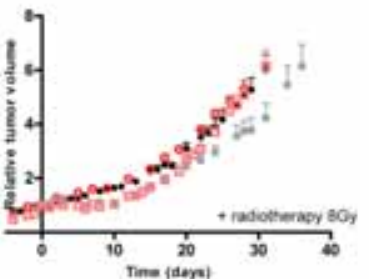

- $\mathrm{NaCl}$

- Nacin.

- thisor

a TH+302T

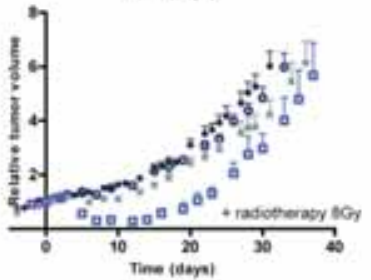

$\mathrm{H} 460$

- Nocios

- nisyo

- thises 05\%

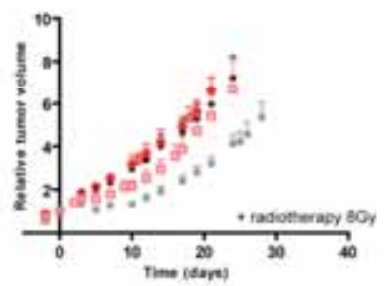

- Naca

- Notrs

- threa

- Theos $\mathrm{n}$

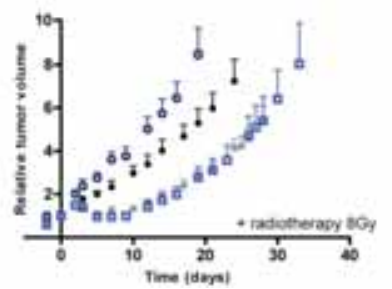

Figure 5.2 The effect of oxygen modification on the combination treatment of TH-302 and radiotherapy. A) Exposing animals to $95 \%$ oxygen (nicotinamide $500 \mathrm{mg} / \mathrm{kg}$ i.p. / carbogen breathing $95 \%$ oxygen, $5 \% \mathrm{CO}_{2}$ ), or $7 \%$ oxygen (residual $\mathrm{N}_{2}$ ) decreases or increases the hypoxic fraction respectively before the start of treatment compared to $21 \%$ oxygen breathing (ambient air). Representative $\left[{ }^{18} \mathrm{~F}\right] \mathrm{HX} 4 \mathrm{PET}$ images with the delineation of the tumor in black and the hypoxic fraction in red. ${ }^{*} P<0.05,{ }^{* * * *} P<0.0001$. Exposing the $B$ ) rhabdomyosarcoma ( $n \geq 7$ ) or C) $\mathrm{H} 460$ ( $n \geq 8$ ) model to modifying oxygen concentrations for the duration of the treatment for 4 and 2.5 hours per day respectively. Data represent mean \pm SEM. 


\section{Hypoxic fraction}

The effect of TH-302 on the hypoxic fraction (HF) in the rhabdomyosarcoma model was assessed using $\left[{ }^{18} \mathrm{~F}\right] \mathrm{HX} 4$ hypoxia PET imaging and revealed a significant decrease from a baseline of $23.1 \% \pm 6.7$ to $2.5 \% \pm 4.2$ after $\mathrm{TH}-302$ treatment $(\mathrm{P}<0.001)$. $\mathrm{NaCl}$ treatment, as expected, did not affect the HF (figure 5.1C). In the $\mathrm{H} 460$ model the HF was assessed immediately after the treatment using histological controls injected with pimonidazole. The HF in subjects treated with TH-302 significantly decreased compared to the control animals ( $\mathrm{NaCl}: 7.8 \% \pm 3.0 ; \mathrm{TH}-302$ : $1.3 \% \pm 0.5$; figure $5.1 \mathrm{D}$ ).

\section{Effect of oxygen modification on the efficacy of TH-302 and radiotherapy}

To investigate if a causal relationship exists between TH-302 efficacy and tumor oxygenation, the amount of oxygen present in the tumor was modified on the days of $\mathrm{TH}-302$ treatment by $7 \%$ or $95 \%$ oxygen breathing to increase or decrease the HF respectively. Oxygen modification was performed 1 day in advance for the rhabdomyosarcoma model in order to assess the effect of this modification on the baseline HF using $\left[{ }^{18} \mathrm{~F}\right] \mathrm{HX} 4-\mathrm{PET}$ imaging. Ambient air breathing animals had a HF of $22.2 \pm 13.8 \%$. A significant reduction in the HF to $2.1 \pm 4.7 \%$ was seen after $95 \%$ oxygen breathing $(P<0.001)$, while 7\% oxygen breathing significantly increased the HF to 29.5 $\pm 14.7 \%$ ( $P=0.029$; figure $5.2 \mathrm{~A}$ ). Exposing rhabdomyosarcoma-bearing rats to increasing oxygen conditions abolished the effect of TH-302 and reduced the T4xSV from 20.4 \pm 3.5 to $15.3 \pm 2.5$ days $(P=0.007$, figure $5.2 \mathrm{~B}$, Supplementary figure $5.3 \mathrm{C}$ and Supplementary Table 5.1) while control animals had an increased T4xSV. Upon combination with $\mathrm{RT}$, the T4xSV of TH-302 treated tumors decreased from $30.8 \pm 5.9$ $(\mathrm{TH}-302+\mathrm{RT})$ to $25.7 \pm 2.9$ days $\left(\mathrm{TH}-302+\mathrm{RT}+95 \% \mathrm{O}_{2}\right)$. This is comparable to the T4XSV of $23.2 \pm 1.7$ days for animals treated with $\mathrm{NaCl}+\mathrm{RT}$ under $95 \% \mathrm{O}_{2}$ conditions resulting in an ER of 1.11 (Supplementary 5.1). Exposing animals to 7\% oxygen breathing resulted in a T4xSV of $22.6 \pm 4.2$ days for TH-302 which is significantly delayed compared to the animals treated with $\mathrm{NaCl}$ (T4xSV: $16.1 \pm 1.9, \mathrm{P}=0.001$ ) although 7\% oxygen treatment itself also had an effect on tumor growth in control animals. In the combination therapy of $7 \%$ oxygen breathing with RT, animals treated with TH-302 had a further reduction in the T4XSV to $35.4 \pm 6.1$ days with an ER of 1.29 compared to the animals treated with $\mathrm{NaCl}$ (Supplementary figure $5.3 \mathrm{C}$ and Supplementary Table 5.1). 


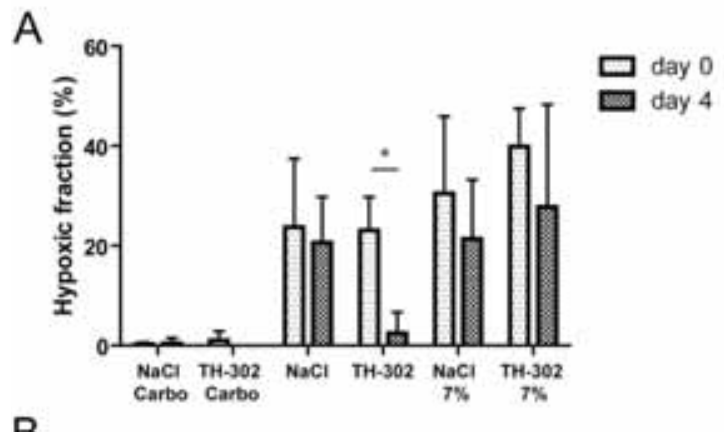

B
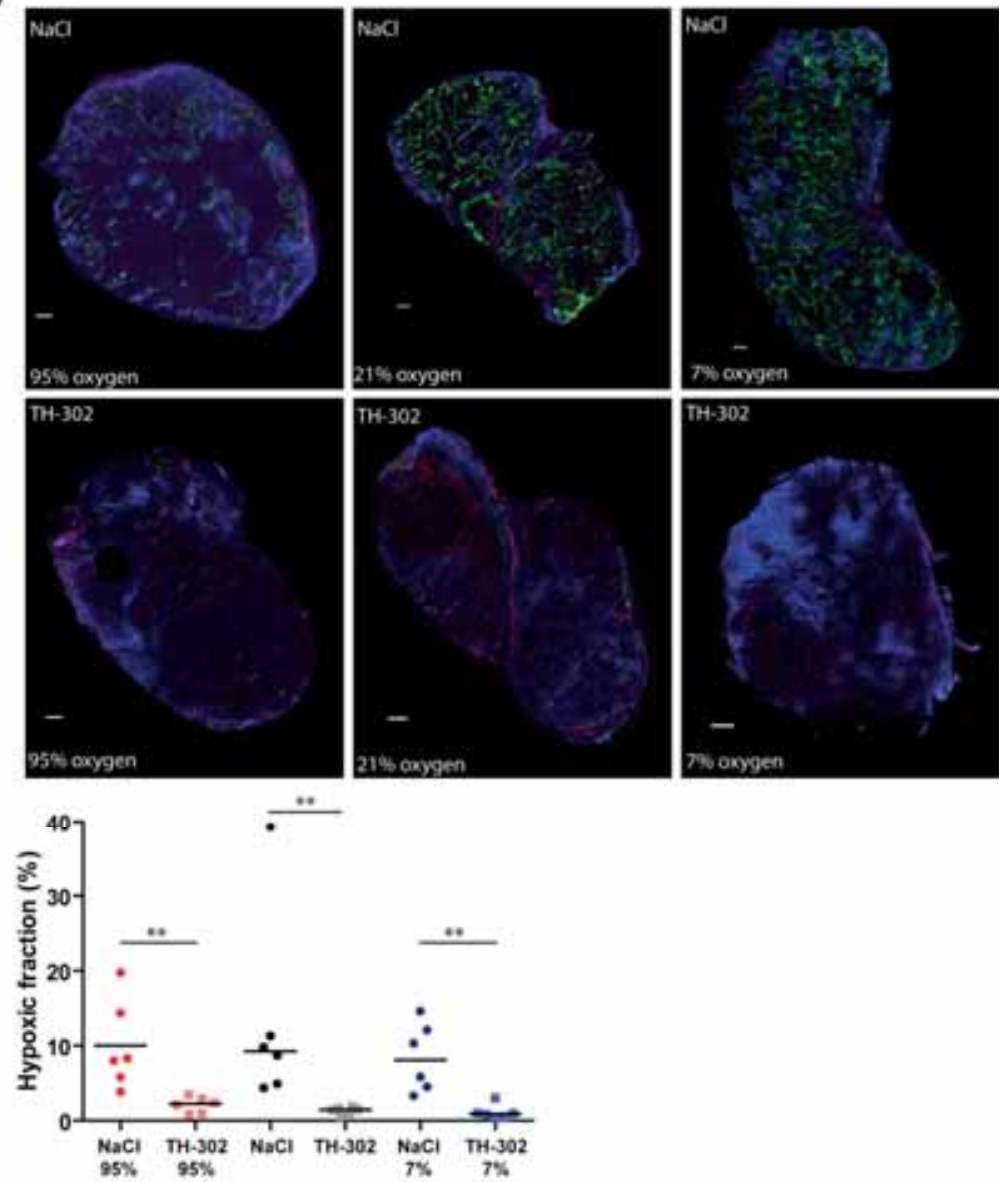

Figure 5.3 The effect of TH-302 treatment and oxygen modification on the hypoxic fraction. A) Hypoxic fraction was measured in the rhabdomyosarcoma model $(n=6)$ using $\left[{ }^{18} \mathrm{~F}\right] \mathrm{HX} 4$ hypoxia PET imaging the day before treatment and the last day of treatment with either control $(\mathrm{NaCl})$ or TH-302 in combination with $95 \%$ oxygen (nicotinamide and $95 \% \mathrm{O}_{2}$ breathing), ambient air or $7 \%$ oxygen. $\mathrm{ND}=$ not detected. $\mathrm{B}$ ) Pimonidazole staining was used to determine the hypoxic fraction after treatment in the $\mathrm{H} 460$ model. Top: a representative image is depicted per group. Bottom: quantification per group $(n=6) .{ }^{*} *<<0.01,{ }^{* * *} p<0.001$ 
Oxygen modification treatments were also applied to the $\mathrm{H} 460$ model where reducing the HF resulted in a decreased T4xSV from $25.2 \pm 4.9(\mathrm{TH}-302+\mathrm{RT})$ to $20.2 \pm 7.0$ (TH$302+\mathrm{RT}+95 \% \mathrm{O}_{2}$ ) for the combination treatment. This decrease was not significant, however. The ER of TH-302 under high oxygen concentration remained stable at 1.50 versus 1.49 at $21 \% \mathrm{O}_{2}$ breathing. The tumor growth rate itself was unaffected by $95 \%$ oxygen breathing (figure 5.2C, Supplementary figure 5.3D and Supplementary Table 5.2). Increasing the HF significantly enlarged the therapeutic potential of $\mathrm{TH}-302$ compared to normal breathing animals $(P=0.011)$, resulting in a T4XSV of $22.7 \pm 7.9$ (T4xSV TH-302 21\% $\mathrm{O}_{2}: 13.6 \pm 4.8$ ). While 7\% oxygen breathing reduced the tumor growth slightly, radiotherapy increased the tumor growth of control animals under this condition. The effect of the TH-302 and RT combination increased to an ER of 2.45 for $\mathrm{TH}-302+\mathrm{RT}$ under low oxygen concentrations versus 1.49 for TH-302 + RT under 21\% $\mathrm{O}_{2}$ concentrations (Supplementary figure 5.3D and Supplementary Table 5.2).

No toxic effects were observed for the different treatments in any of the groups as monitored by following changes in body weight (Supplementary figure 5.4A\&B).

\section{Oxygen modification and hypoxic fraction}

To assess the effect of TH-302 treatment in combination with oxygen modification on the HF, a $\left[{ }^{18} \mathrm{~F}\right] \mathrm{HX} 4$ scan was acquired before and after treatment on the rhabdomyosarcoma histological control animals. The HF of ambient air breathing animals decreased from $23 \% \pm 6.7$ at baseline to $2.5 \% \pm 4.2$ after $\mathrm{TH}-302$ treatment. For $95 \%$ oxygen breathing animals the HF was low before the start of the treatment, and this remained unchanged after either $\mathrm{NaCl}$ or $\mathrm{TH}-302$ administration (figure 5.3A). The spread in $\mathrm{HF}$ of $7 \%$ oxygen breathing animals was very large. On average the $\mathrm{HF}$ after treatment was lower than before treatment independent of $\mathrm{NaCl}$ or $\mathrm{TH}-302$ treatment although this was not significant.

The HF in the $\mathrm{H} 460$ model was determined after the last $\mathrm{TH}-302$ injection using pimonidazole staining. TH-302 treatment significantly reduced the HF compared to the control animals (figure 5.1D \& 5.3B). The different oxygen breathing conditions revealed a similar pattern; in combination with $95 \%$ oxygen breathing control animals had a HF of $10.0 \% \pm 5.9$ while animals treated with $\mathrm{TH}-302$ had a HF of $2.1 \% \pm 1.0$. Animals exposed to low oxygen concentrations in combination with $\mathrm{NaCl}$ had a $\mathrm{HF}$ of $8.4 \% \pm 4.5$ which was lower in the animals treated with $\mathrm{TH}-302(1.1 \% \pm 1.0)$. Furthermore, $\mathrm{TH}-302$-treated tumors had a decreased necrotic fraction, although this 

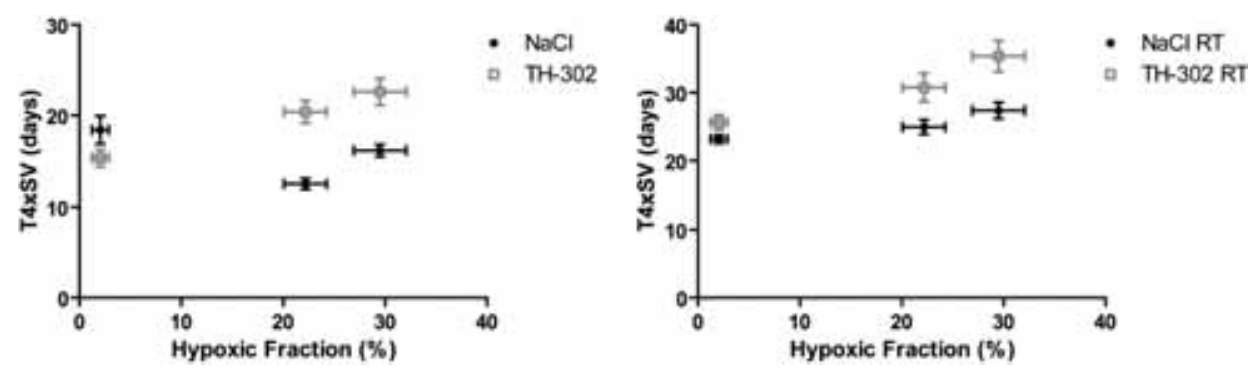

Figure 5.4 The association between the pretreatment $\left[{ }^{18} \mathrm{~F}\right] \mathrm{HX} 4$ based hypoxic fraction and the treatment outcome in rhabdomyosarcoma tumors. Hypoxic fraction (\%) as measured before the start of the treatment plotted to the time to reach 4 times the start volume (T4xSV) for the control group ( $\mathrm{NaCl}$ ) and animals treated with $\mathrm{TH}-302$ either with or without radiotherapy. Hypoxic fraction is determined using $\left[{ }^{18} \mathrm{~F}\right] \mathrm{HX} 4$ hypoxia PET imaging after $95 \%$ oxygen breathing $n=32$, ambient air breathing $n=43$ and $7 \%$ oxygen breathing $\mathrm{n}=33$. The T4xSV is calculated for $\mathrm{NaCl}$ for all oxygen concentrations using 8 animals, $\mathrm{TH}-30295 \%$ oxygen $n=7$, ambient air and $7 \%$ oxygen $n=8$. Data represent mean \pm SEM.

was only significant when animals were exposed to $21 \%$ oxygen. No differences were observed in the relative vessel area or perfusion (Supplementary figure 5.5).

Furthermore, we investigated whether the HF at the start of the therapy was associated with the treatment outcome expressed as T4XSV. The T4xSV for TH-302 treated tumors increased with increasing $\mathrm{HF}$ at onset meaning that $95 \%$ oxygen breathing animals reached their endpoint first, followed by ambient air and then $7 \%$ oxygen breathing animals (figure 5.4). The control animals, with or without radiotherapy, did not show this association.

\section{Discussion}

This study demonstrates the combination efficacy of the hypoxia-activated prodrug TH-302 with radiotherapy in two preclinical tumor models, which was causally related to the tumor oxygen status. Additionally, the $\left[{ }^{18} \mathrm{~F}\right] \mathrm{HX} 4$ determined hypoxic fraction (HF) was associated with the treatment outcome.

Pharmacokinetic studies in non-tumor bearing rats showed no adverse effects when the animals were treated with $\mathrm{TH}-302$ (17). Although rhabdomyosarcoma-bearing rats showed no adverse effect after a $25 \mathrm{mg} / \mathrm{kg}$ TH-302 dose, dose-dependent adverse effects, such as a significant drop in body weight, diarrhea and general malaise, were observed after higher dosing. Initial clinical studies also reported some adverse effects 
with skin and mucosal toxicities being dose limiting, while common adverse events were nausea, skin rash, fatigue and vomiting $(5,6)$.

In this study two different methods were used to assess the HF: noninvasive, clinically used $\left[{ }^{18} \mathrm{~F}\right] \mathrm{HX} 4$ PET imaging $(11,13)$, and immunohistochemistry of pimonidazole adducts (9). While $\left[{ }^{18} \mathrm{~F}\right] \mathrm{HX} 4$ hypoxia PET imaging represents the whole tumor in a noninvasive, reproducible, three dimensional manner, immunohistochemical stainings can, in addition to hypoxia, extract more tumor microenvironmental information from the same tumor section. In both techniques, $\left[{ }^{18} \mathrm{~F}\right] \mathrm{HX} 4$ and pimonidazole, are reduced under low oxygen concentrations (18) and a significant co-localization relationship was demonstrated at the tumor sub-regional level (9). Although monitored by different techniques, these data indicate that $\mathrm{TH}-302$ has the same reducing effect on the HF in both tumor models. Furthermore, based on these data, $\left[{ }^{18} \mathrm{~F}\right] \mathrm{HX} 4$ imaging could be used as biomarker of response in a window-of-opportunity clinical trial. By performing a pretreatment scan, the initial tumor HF can be determined, followed by a single injection of $\mathrm{TH}-302$. A post-treatment $\left[{ }^{18} \mathrm{~F}\right] \mathrm{HX} 4$ hypoxia scan can assess whether there is a response in $\mathrm{HF}$ to this hypoxia-activated prodrug, without interference of other anticancer therapies. This new approach is designed to test new molecular entities in a clinical trial while being cost and patient efficient $(19,20)$.

A correlation has previously been reported between the HF and the tumor growth inhibition in xenograft models (3). This endorses our findings of a pronounced effect of $\mathrm{TH}-302$ since the preclinical tumor models in this study were observed to have respectively somewhat higher and lower HF than the reported HF of $18.6 \%$ for rhabdomyosarcoma (15) and $16.3 \%$ for H460 (3). Although the HF in tumors was significantly decreased after $\mathrm{TH}-302$ treatment, not all hypoxic cells were eliminated, as observed in both tumor models. The remaining hypoxic cells might be resistant, unreachable by $\mathrm{TH}-302$, or caused by cycling hypoxia. In addition to the decrease in the $\mathrm{HF}$, this study shows a decrease in the necrotic fraction of the TH-302 treated tumors. This indicates that the dead cells are resorbed, which is supported by the stagnation in tumor growth after 3 days of TH-302 treatment. Other microenvironmental characteristics like relative vessel area and perfusion were not affected by $\mathrm{TH}-302$ treatment, suggesting that the tumor maintains its vasculature. This is in agreement with previously published results on solid tumors (3).

Although TH-302 exhibits anti-tumor effects as a monotherapy, it has been shown that its therapeutic efficacy increases when combined with conventional anticancer 
therapies mainly targeting the non-hypoxic cells. Based on favorable outcomes of two clinical phase II trials $(7,8)$, phase III trials in metastatic or locally advanced unresectable pancreatic adenocarcinoma (NCT01746979) and advanced soft-tissue sarcoma (NCT01440088) are currently ongoing. However, to our knowledge, no study has investigated the combination treatment of TH-302 with radiotherapy and $\left[{ }^{18} \mathrm{~F}\right] \mathrm{HX} 4$ hypoxia imaging. This combination is thought to be effective especially since RT can be locally applied, specifically targeting the tumor while preserving normal tissue. In this study we show that this combination is effective and causes a delayed tumor growth and increased T4XSV for both investigated tumor models, confirming the hypothesis that the combination therapy of TH-302 and RT will lead to an enhanced therapeutic effect. In the rhabdomyosarcoma tumor model, TH-302 treatment was combined with a single dose of 4,8 and $12 \mathrm{~Gy}$ of RT resulting in a dose dependent effect. In subsequent studies the single RT dose of $8 \mathrm{~Gy}$ was used, reasoning that the regrowth of the tumor solely depends on hypoxic cells (21), providing a basis for $\mathrm{TH}-302$ efficacy. This approach is different from clinical practice where fractionated radiotherapy schedules are used. By applying 2Gy fractions to the tumor, reoxygenation occurs and the HF gradually decreases $(22,23)$. We speculate that the combination of $\mathrm{TH}-302$ with fractionated radiotherapy would also increase the therapeutic effect of the RT since the $\mathrm{HF}$ is reduced by the pretreatment of TH-302, increasing the potential of RT.

In this study we further wanted to elucidate if TH-302 efficacy is dependent on the tumor oxygen status. Exposing animals to either nicotinamide and carbogen or $7 \%$ oxygen breathing has been demonstrated to be effective in modulating the HF in tumors $(9,24,25)$. Altering oxygen breathing before the TH-302 treatment did modify the tumor $\mathrm{HF}$ in rhabdomyosarcoma animals as measured by $\left[{ }^{18} \mathrm{~F}\right] \mathrm{HX} 4$. However, in the H460 model, the HF was only determined after TH-302 treatment and at this point no differences were observed in control animals of the various oxygen modifications. A possible explanation would be that the mice adapted to the oxygen breathing schedule, preventing the tumor HF from changing, which has been observed for mice exposed to long-term carbogen breathing $(26,27)$. In the rhabdomyosarcoma model, the tumor growth of control animals was significantly reduced upon oxygen modification as well as in the mice exposed to 7\% oxygen breathing mice. No effect on tumor growth was observed after oxygen modification in another study using $\mathrm{H} 460$ tumors exposed to $95 \%$ or $10 \%$ oxygen breathing (3). This unexpected finding could possibly be explained by the stress induced by the exposure to the oxygen modifications, although TH-302 or RT treatment groups did not seem to be influenced by this. However, by calculating the enhancement ratio these oxygen modification 
effects are taken into account. Despite these effects on growth delay a positive effect of the therapy is observed. Radiotherapy was applied 1 hour after carbogen breathing without any beneficial effect. This can be explained by the fact that tumor oxygen concentrations return to pre-carbogen levels within 1 minute after stopping carbogen treatment as detected by Eppendorf electrode measurements (28). Furthermore, clinical studies have shown that the presence of hypoxia and the pretreatment selection of patients with hypoxic tumors is essential for the combination of nicotinamide administration and carbogen breathing to be effective $(29,30)$. Breathing low oxygen concentrations reduced the effect of radiotherapy in the H460 model, indicating that, although not detected on pimonidazole immunohistochemical staining, low oxygen concentrations counteracted the irradiation. The effect of $\mathrm{TH}-302$ is abolished by carbogen breathing in the rhabdomyosarcoma model independent of radiotherapy. This can be explained by the reduced HF leaving almost no cells present to convert $\mathrm{TH}-302$ into its cytotoxic metabolite. For the $\mathrm{H} 460$ model the HF did not decrease upon carbogen breathing what reflects in the unchanged tumor growth compared to control tumors. Upon radiotherapy however, there is a trend towards a faster tumor growth that also indicating abolishment of the TH-302 efficacy. In tumors with an enlarged HF, TH-302 caused a slight, non-significant, delay in tumor growth compared to TH-302 under normal air conditions. Moreover, TH-302 decreased the HF to almost zero under ambient air conditions, while with $7 \%$ oxygen breathing the HF is still $28 \%$. Although this result could be caused by the counteracting effects of $\mathrm{TH}-302$ reducing the $\mathrm{HF}$ and the $7 \%$ oxygen breathing increasing the $\mathrm{HF}$, we speculate that it is caused by a limited availability of TH-302 to target all hypoxic cells. In H460 tumors, 7\% oxygen breathing resulted in an increased therapeutic effect with an enhancement ratio of 2.2 for $\mathrm{TH}-302$ alone and 2.5 for the combination therapy of TH-302 and RT. This result demonstrates that when sufficient TH-302 is present, more $\mathrm{TH}-302$ is reduced upon low oxygen concentrations, causing an increased cytotoxicity.

A causal relation between the pretreatment $\mathrm{HF}$ measured by $\left[{ }^{18} \mathrm{~F}\right] \mathrm{HX} 4$ and the $\mathrm{TH}-302$ treatment outcome was observed. These results indicate that pretreatment evaluation of hypoxia could be a useful tool in selecting tumors that benefit from the additional hypoxia targeting treatment. This hypoxia-based patient selection could also be used in other therapy strategies for instance to target hypoxic subvolumes by escalate radiation dose (31). Furthermore this information could be implemented in decisionsupport systems to predict tumor response and optimize patient therapy (32). These applications demonstrate the importance of gaining pretreatment information by hypoxia imaging. 


\section{Conclusion}

This study demonstrates that TH-302 treatment together with conventional radiotherapy is a promising combination with an increased therapeutic potential, and warrants further testing. Furthermore, detecting the tumor hypoxic fraction by $\left[{ }^{18} \mathrm{~F}\right] \mathrm{HX} 4$ PET imaging may allow the ability to predict which patients will benefit most from the hypoxia targeted $\mathrm{TH}-302$ treatment and gives the possibility to noninvasively monitor TH-302 efficacy in the context of window-of-opportunity trials. Based on this preclinical study we suggest a clinical trial for treating patients with the combination of TH-302 and radiotherapy while monitoring the hypoxic fraction before and during the treatment.

\section{Grant support}

Authors acknowledge financial support from the QuIC-ConCePT project, which is partly funded by EFPI A companies and the Innovative Medicine Initiative Joint Undertaking (IMI JU) under Grant Agreement No. 115151. Authors also acknowledge financial support from the EU 6th and 7th framework program (METOXIA, EURECA, ARTFORCE), Kankeronderzoekfonds Limburg from the Health Foundation Limburg and the Dutch Cancer Society (KWF UM 2011-5020, KWF-MAC 2011-4970, KWF MAC 2013-6425, KWF MAC 2013-6089). The micrographs in this paper were taken with a confocal spinning disk microscope financed by The Netherlands Organization for Scientific Research (NWO), grant number 911-06-003. 


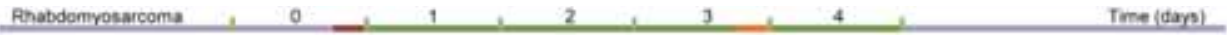

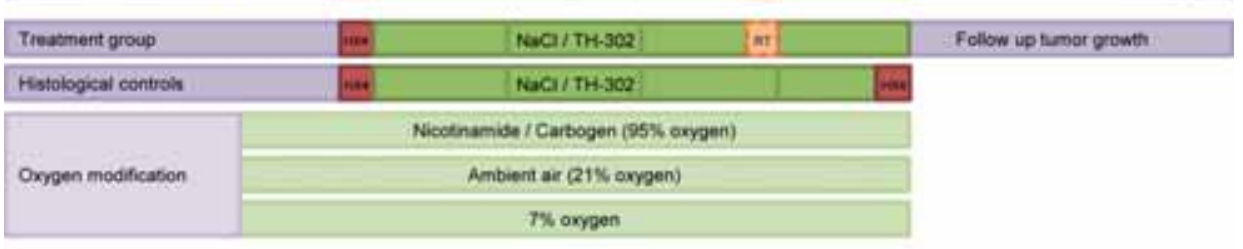

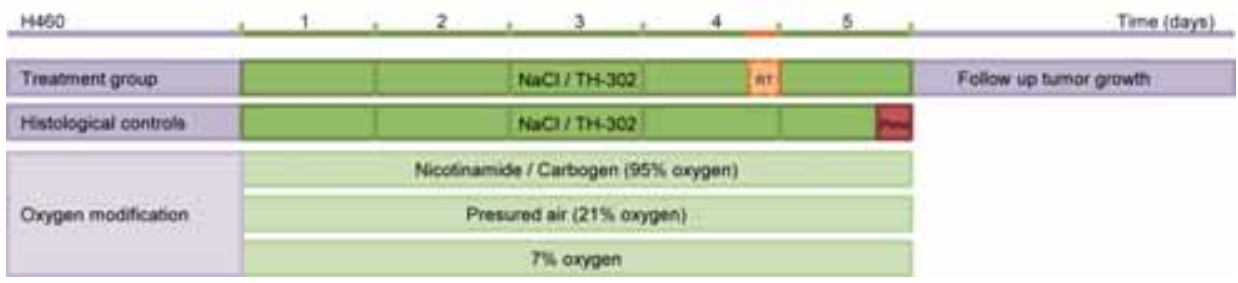

Supplementary figure $5.1 \mathrm{~A}$ ) Treatment schedule for the rhabdomyosarcoma and $\mathrm{H} 460$ models. All animals were randomized over either the treatment or the control group. These groups were both treated with either $\mathrm{NaCl}$ or TH-302 (rhabdomysarcoma $25 \mathrm{mg} / \mathrm{kg}, \mathrm{H} 46050 \mathrm{mg} / \mathrm{kg}$ ). Rhabdomyosarcoma bearing animals started with oxygen modification at day 0 and a $\left[{ }^{18} \mathrm{~F}\right] \mathrm{HX} 4$ hypoxia PET scan. For histological controls only, this scan was repeated on day 4. Animals from the treatment group were exposed to radiotherapy (RT) at the end of treatment day 3 and their tumor volume was monitored until 4 times start volume was reached. H460 tumor bearing animals from the histological control group were injected with pimonidazole and Hoechst on the last treatment day and sacrificed. Animals from the treatment group were exposed to radiotherapy at day 4 and monitored until a tumor volume of 4 times start treatment volume was reached.
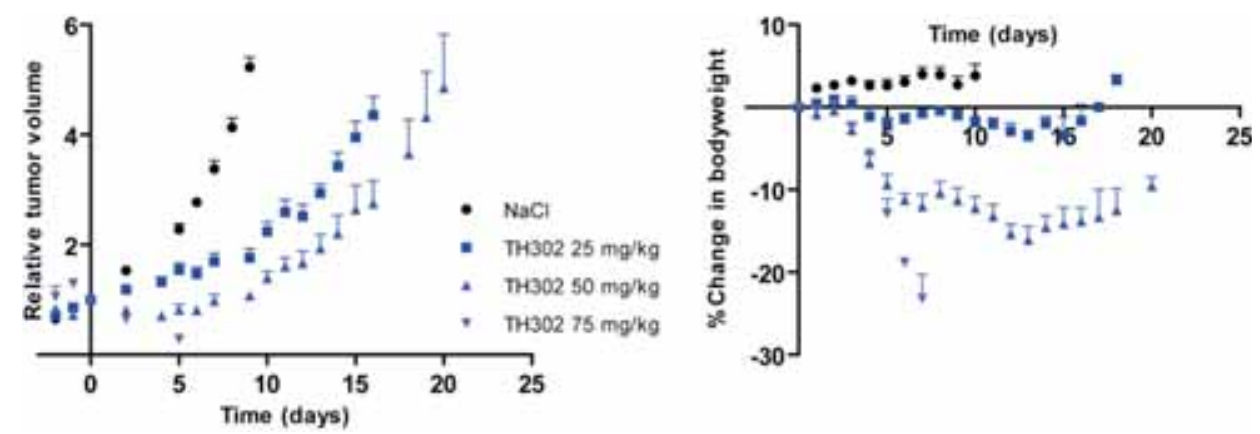

Supplementary figure $\mathbf{5 . 2}$ Optimal TH-302 dose assessment for rhabdomyosarcoma bearing rats. Animals were treated on 4 consecutive days with either the control $(\mathrm{NaCl}), 25,50$ or $75 \mathrm{mg} / \mathrm{kg} \mathrm{TH}-302$. Tumor volume (A) and body weight (B) was monitored over time. Data represent mean $\pm S E M$, for all groups $n=9$. 
A

Rhabdomyosarcoma

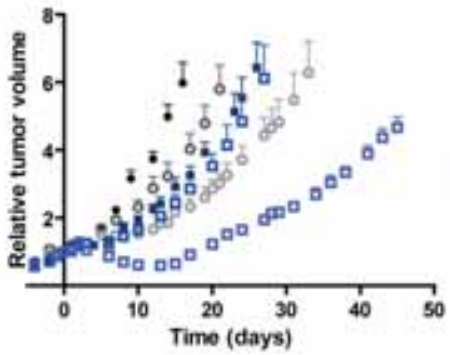

C

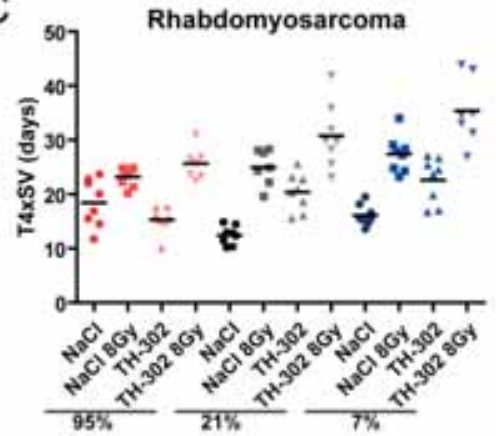

B

- $\mathrm{NaCl}$

- $\mathrm{NaCl} 4 \mathrm{~Gy}$

- $\mathrm{NaCl} 12 \mathrm{~Gy}$

- TH.302

- TH-302 4Gy

a TH302 12Gy
Rhabdomyosarcoma

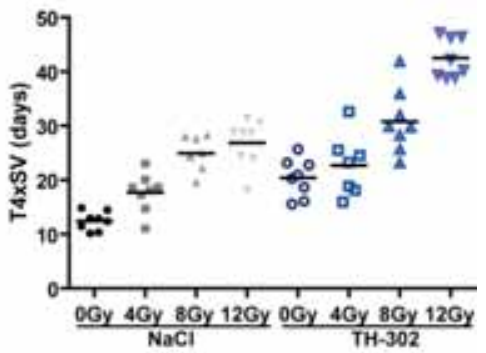

D

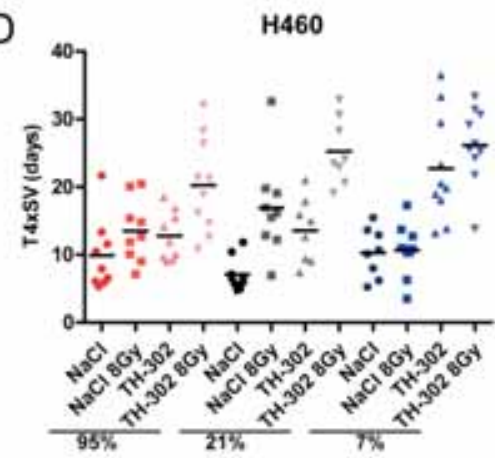

Supplementary figure $\mathbf{5 . 3}$ A) Tumor growth of rhabdomyosarcoma bearing animals $(n=8)$ treated with $\mathrm{TH}$ $302(25 \mathrm{mg} / \mathrm{kg})$ in combination with 4,8 or $12 \mathrm{~Gy}$ of RT. Data represent mean \pm SEM. B) T4xSV for rhabdomyosarcoma bearing animals treated with TH-302 in combination with RT 0, 4, 8 or 12Gy. C) T4xSV for rhabdomyosarcoma bearing animals exposed to different oxygen concentrations in combination with $\mathrm{TH}$ 302 and RT. D) T4xSV for $\mathrm{H} 460$ bearing animals exposed to different oxygen concentrations in combination with TH-302 and RT. 
A
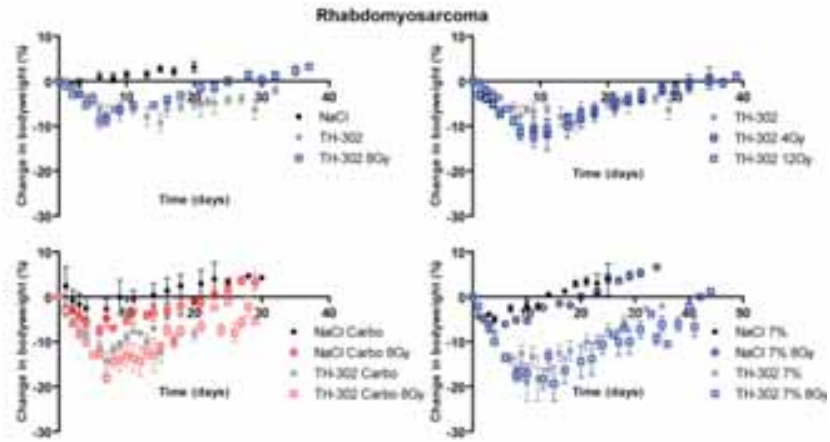

B
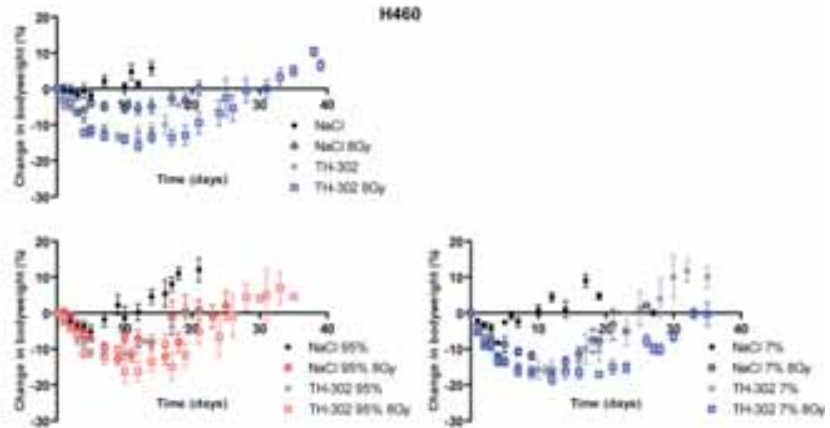

Supplementary figure 5.4 Body weight was monitored during and after treatment for both rhabdomyosarcoma (A) and $\mathrm{H} 460$ (B) tumor-bearing animals. The change in body weight is represented in percentage. Data represent mean \pm SEM.

A

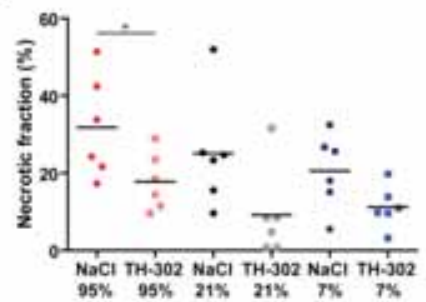

C

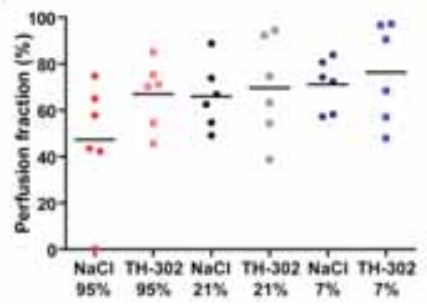

B

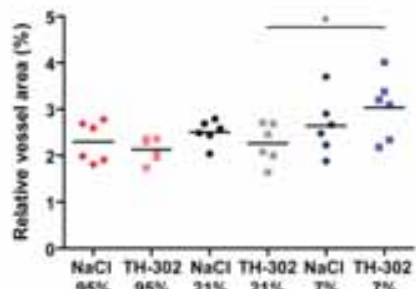

D

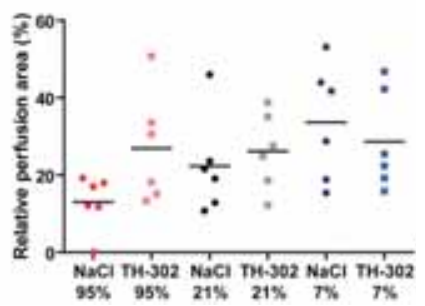

Supplementary figure 5.5 Quantification of $\mathrm{H} 460$ histological staining for A) necrotic fraction (NF), B) relative vessel area (RVA), C) perfusion fraction (PF) and D) relative perfusion area (RPA). Data represent mean \pm SEM 


\begin{tabular}{ccccc}
\hline Rhabdomyosarcoma & $\begin{array}{c}\text { T4xSV } \\
\text { NaCl }\end{array}$ & $\begin{array}{c}\text { T4xSV } \\
\text { TH-302 }\end{array}$ & P-value & ER \\
\hline 21\% & $12,4 \pm 1,7$ & $20,4 \pm 3,5$ & $<0,0001$ & 1,64 \\
\hline 21\% RT 4Gy & $17,7 \pm 3,6$ & $22,7 \pm 5,6$ & 0,0583 & 1,28 \\
21\% RT 8Gy & $24,9 \pm 3,0$ & $30,8 \pm 5,9$ & 0,0261 & 1,23 \\
\hline 21\% RT 12Gy & $26,8 \pm 4,4$ & $42,5 \pm 3,6$ & $<0,0001$ & 1,58 \\
\hline 95\% & $18,4 \pm 4,4$ & $15,3 \pm 2,5$ & 0,1234 & 0,83 \\
95\% RT & $23,2 \pm 1,7$ & $25,7 \pm 2,9$ & 0,0601 & 1,11 \\
7\% & $16,1 \pm 1,9$ & $22,6 \pm 4,2$ & 0,0013 & 1,40 \\
\hline 7\%RT & $27,4 \pm 3,4$ & $35,4 \pm 6,1$ & 0,0072 & 1,29 \\
\hline
\end{tabular}

Supplementary Table 5.1 Time to reach 4 times start volume per treatment group for rhabdomyosarcoma bearing animals. Data represent mean $\pm S D, p$-value and sensitization enhancement ratio.

\begin{tabular}{ccccc}
\hline H460 & $\begin{array}{c}\text { T4xSV } \\
\text { NaCl }\end{array}$ & $\begin{array}{c}\text { T4xSV } \\
\text { TH-302 }\end{array}$ & P-value & ER \\
\hline 21\% & $7,1 \pm 2,4$ & $13,6 \pm 4,8$ & 0,0027 & 1,9 \\
\hline $\mathbf{2 1 \%}$ RT & $16,9 \pm 7,1$ & $25,2 \pm 4,9$ & 0,0136 & 1,49 \\
\hline 95\% & $9,9 \pm 5,2$ & $12,8 \pm 3,6$ & 0,1917 & 1,29 \\
\hline 95\% RT & $13,5 \pm 4,6$ & $20,2 \pm 7,0$ & 0,0260 & 1,5 \\
\hline 7\% & $10,3 \pm 3,6$ & $22,7 \pm 7,9$ & 0,0009 & 2,21 \\
\hline 7\%RT & $10,7 \pm 3,8$ & $26,2 \pm 5,6$ & $<0,0001$ & 2,45 \\
\hline
\end{tabular}

Supplementary Table 5.2 Time to reach 4 times start volume per treatment group for $\mathrm{H} 460$ bearing animals. Data represent mean \pm SD, $p$-value and sensitization enhancement ratio. 


\section{References}

1. Horsman MR, Mortensen LS, Petersen JB, Busk M, Overgaard J. Imaging hypoxia to improve radiotherapy outcome. Nature reviews Clinical oncology. 2012;9:674-87.

2. Vaupel P, Mayer A. Hypoxia in cancer: significance and impact on clinical outcome. Cancer Metastasis Rev. 2007;26:225-39.

3. Sun JD, Liu Q, Wang J, Ahluwalia D, Ferraro D, Wang Y, et al. Selective tumor hypoxia targeting by hypoxia-activated prodrug TH-302 inhibits tumor growth in preclinical models of cancer. Clin Cancer Res. 2012;18:758-70.

4. Liu Q, Sun JD, Wang J, Ahluwalia D, Baker AF, Cranmer LD, et al. TH-302, a hypoxia-activated prodrug with broad in vivo preclinical combination therapy efficacy: optimization of dosing regimens and schedules. Cancer Chemother Pharmacol. 2012;69:1487-98.

5. Weiss GJ, Infante JR, Chiorean EG, Borad MJ, Bendell JC, Molina JR, et al. Phase 1 study of the safety, tolerability, and pharmacokinetics of $\mathrm{TH}-302$, a hypoxia-activated prodrug, in patients with advanced solid malignancies. Clin Cancer Res. 2011;17:2997-3004.

6. Ganjoo KN, Cranmer LD, Butrynski JE, Rushing D, Adkins D, Okuno SH, et al. A phase I study of the safety and pharmacokinetics of the hypoxia-activated prodrug $\mathrm{TH}-302$ in combination with doxorubicin in patients with advanced soft tissue sarcoma. Oncology. 2011;80:50-6.

7. Chawla SP, Cranmer LD, Van Tine BA, Reed DR, Okuno SH, Butrynski JE, et al. Phase II Study of the Safety and Antitumor Activity of the Hypoxia-Activated Prodrug TH-302 in Combination With Doxorubicin in Patients With Advanced Soft Tissue Sarcoma. J Clin Oncol. 2014.

8. Borad MJ, Reddy SG, Bahary N, Uronis HE, Sigal D, Cohn AL, et al. Randomized Phase II Trial of Gemcitabine Plus TH-302 Versus Gemcitabine in Patients With Advanced Pancreatic Cancer. J Clin Oncol. 2014.

9. Dubois L, Lieuwes NG, Janssen MH, Peeters WJ, Windhorst AD, Walsh JC, et al. Preclinical evaluation and validation of [18F]HX4, a promising hypoxia marker for PET imaging. Proc Natl Acad Sci U S A. 2011;108:14620-5.

10. Zegers CM, van Elmpt W, Wierts R, Reymen B, Sharifi H, Ollers MC, et al. Hypoxia imaging with [(1)(8)F]HX4 PET in NSCLC patients: defining optimal imaging parameters. Radiother Oncol. 2013;109:58-64.

11. Zegers CM, van Elmpt W, Reymen B, Even AJ, Troost EG, Ollers MC, et al. In Vivo Quantification of Hypoxic and Metabolic Status of NSCLC Tumors Using [18F]HX4 and [18F]FDG-PET/CT Imaging. Clin Cancer Res. 2014.

12. Rischin D, Hicks RJ, Fisher R, Binns D, Corry J, Porceddu S, et al. Prognostic significance of [18F]misonidazole positron emission tomography-detected tumor hypoxia in patients with advanced head and neck cancer randomly assigned to chemoradiation with or without tirapazamine: a substudy of Trans-Tasman Radiation Oncology Group Study 98.02. J Clin Oncol. 2006;24:2098-104.

13. Peeters SG, Zegers CM, Lieuwes NG, van Elmpt W, Eriksson J, van Dongen GA, et al. A Comparative Study of the Hypoxia PET Tracers [F]HX4, [F]FAZA, and [F]FMISO in a Preclinical Tumor Model. Int J Radiat Oncol Biol Phys. 2014.

14. van Loon J, Janssen $\mathrm{MH}$, Ollers $\mathrm{M}$, Aerts HJ, Dubois L, Hochstenbag $\mathrm{M}$, et al. PET imaging of hypoxia using [18F]HX4: a phase I trial. Eur J Nucl Med Mol Imaging. 2010;37:1663-8.

15. Dubois L, Landuyt W, Haustermans K, Dupont P, Bormans G, Vermaelen P, et al. Evaluation of hypoxia in an experimental rat tumour model by [(18)F]fluoromisonidazole PET and immunohistochemistry. $\mathrm{Br}$ J Cancer. 2004;91:1947-54. 
16. Edelstein A, Amodaj N, Hoover K, Vale R, Stuurman N. Computer control of microscopes using microManager. Curr Protoc Mol Biol. 2010;Chapter 14:Unit14 20.

17. Jung D, Lin L, Jiao H, Cai X, Duan JX, Matteucci M. Pharmacokinetics of TH-302: a hypoxically activated prodrug of bromo-isophosphoramide mustard in mice, rats, dogs and monkeys. Cancer Chemother Pharmacol. 2012;69:643-54.

18. Wilson WR, Hay MP. Targeting hypoxia in cancer therapy. Nat Rev Cancer. 2011;11:393-410.

19. Fass L. Imaging and cancer: a review. Mol Oncol. 2008;2:115-52.

20. Orloff J, Douglas F, Pinheiro J, Levinson S, Branson M, Chaturvedi P, et al. The future of drug development: advancing clinical trial design. Nat Rev Drug Discov. 2009;8:949-57.

21. Hill RP, Bush RS, Yeung P. The effect of anaemia on the fraction of hypoxic cells in an experimental tumour. Br J Radiol. 1971;44:299-304.

22. Stanley JA, Shipley WU, Steel GG. Influence of tumour size on hypoxic fraction and therapeutic sensitivity of Lewis lung tumour. Br J Cancer. 1977;36:105-13.

23. Wouters BG, Brown JM. Cells at intermediate oxygen levels can be more important than the "hypoxic fraction" in determining tumor response to fractionated radiotherapy. Radiat Res. 1997;147:541-50.

24. Horsman MR, Overgaard J. Preclinical studies on how to deal with patient intolerance to nicotinamide and carbogen. Radiotherapy and oncology : journal of the European Society for Therapeutic Radiology and Oncology. 2004;70:301-9.

25. Troost EG, Laverman P, Kaanders JH, Philippens M, Lok J, Oyen WJ, et al. Imaging hypoxia after oxygenation-modification: comparing [18F]FMISO autoradiography with pimonidazole immunohistochemistry in human xenograft tumors. Radiother Oncol. 2006;80:157-64.

26. Hou H, Dong R, Lariviere JP, Mupparaju SP, Swartz HM, Khan N. Synergistic combination of hyperoxygenation and radiotherapy by repeated assessments of tumor pO2 with EPR oximetry. J Radiat Res. 2011;52:568-74.

27. Khan N, Li H, Hou H, Lariviere JP, Gladstone DJ, Demidenko E, et al. Tissue pO2 of orthotopic 9L and C6 gliomas and tumor-specific response to radiotherapy and hyperoxygenation. Int J Radiat Oncol Biol Phys. 2009;73:878-85.

28. Martin L, Lartigau E, Weeger P, Lambin P, Le Ridant AM, Lusinchi A, et al. Changes in the oxygenation of head and neck tumors during carbogen breathing. Radiother Oncol. 1993;27:123-30.

29. Janssens GO, Rademakers SE, Terhaard CH, Doornaert PA, Bijl HP, van den Ende P, et al. Accelerated radiotherapy with carbogen and nicotinamide for laryngeal cancer: results of a phase III randomized trial. J Clin Oncol. 2012;30:1777-83.

30. Schuuring J, Bussink J, Bernsen HJ, Peeters W, van der Kogel AJ. Effect of carbogen breathing on the radiation response of a human glioblastoma xenograft: analysis of hypoxia and vascular parameters of regrowing tumors. Strahlenther Onkol. 2006;182:408-14.

31. Bentzen SM, Gregoire V. Molecular imaging-based dose painting: a novel paradigm for radiation therapy prescription. Semin Radiat Oncol. 2011;21:101-10.

32. Lambin P, van Stiphout RG, Starmans MH, Rios-Velazquez E, Nalbantov G, Aerts HJ, et al. Predicting outcomes in radiation oncology--multifactorial decision support systems. Nat Rev Clin Oncol. 2013;10:27-40. 


\section{Chapter 6}

\section{Specific inhibition of Carbonic Anhydrase $\mathrm{IX}$ activity enhances the in vivo therapeutic effect of tumor irradiation}

Published in: Radiother Oncol. 2011;99:424-31

Ludwig Dubois, Sarah Peeters, Natasja G. Lieuwes, Nele Geusens, Anne Thiry, Simon Wigfield, Fabrizio Carta, Alan Mcintyre, Andrea Scozzafava, Jean-Michel Dogné, Claudiu T. Supuran, Adrian L. Harris, Bernard Masereel and Philippe Lambin 


\section{Abstract}

Background and Purpose: Carbonic anhydrase (CA) IX expression is increased upon hypoxia and has been proposed as a therapeutic target since it has been associated with poor prognosis, tumor progression and $\mathrm{pH}$ regulation. The aim of this study was to evaluate the antitumor activity of a high CAIX-affinity indanesulfonamide (11c) combined with irradiation, compared with the general CA inhibitor acetazolamide (AZA).

Material and M ethods: HT-29 carcinoma cells with or without (genetic knockdown, KD) CAIX expression were incubated with 11c/AZA under different oxygen levels and proliferation, apoptosis and radiosensitivity were evaluated. 11c/AZA was administered intravenously (1x/day; 5 days) to tumor-bearing mice and tumor irradiation (10 Gy) was performed at day 3 of the injection period. Tumor growth and potential treatment toxicity were monitored ( $3 x /$ week).

Results: Treatment with 11c/AZA alone resulted in tumor regression, which was further increased in CAIX expressing cells by combining 11c with irradiation. AZA demonstrated also an additional effect in the KD tumors when combined with irradiation. CAIX inhibition in vitro significantly reduced proliferation and increased apoptosis upon hypoxia exposure without affecting intrinsic radiosensitivity.

Conclusions: Specific inhibition of CAIX activity enhanced the effect of tumors irradiation and might, therefore, be an attractive strategy to improve overall cancer treatment. 


\section{Introduction}

High tumoral carbonic anhydrase (CA) IX expression has been associated with poor prognosis, tumor progression and aggressiveness $(1,2)$. CAIX is a dimeric glycoprotein, contains an HRE element essential for its transcriptional activation upon hypoxia by HIF- $1 \alpha$ and regulates tumor $\mathrm{pH}$ by catalyzing the reversible hydration of carbon dioxide to bicarbonate and a proton $(2,3)$. Since the active site of CAIX resides in the extracellular space, it contributes to the acidification of the extracellular environment during hypoxia (4). Furthermore, it has been shown that CAIX plays an important role in maintaining a neutral intracellular $\mathrm{pH}$ within tumors. The bicarbonate ion resulting from the CAIX catalyzed reaction can be imported into the cytoplasm by exchange with a chloride anion to serve as substrate for intracellular carbonic anhydrases such as CAll in order to neutralize intracellular protons (5). Since CAIX is implicated in both extra and intracellular $\mathrm{pH}$ regulation, it has been proposed as a potential therapeutic target.

A possible approach to target CAIX would be via inhibiting its enzymatic activity with specific pharmacological inhibitors (6). Previously, we have demonstrated that binding of sulfonamides to CAIX requires both its expression and its activation and this occurs only during hypoxia (7). Also in animal models, sulfonamide accumulation was proven to be dependent on the tumor oxygenation (8). Administration of membraneimpermeable derivatives of acetazolamide, a general carbonic anhydrase inhibitor, resulted in tumor growth inhibition (9). Aromatic sulfonamides were able to reduce tumor cell proliferation and intracellular $\mathrm{pH}$, accompanied with an increase in ceramide-mediated apoptotis (10). Recently, treatment of mammary tumor-bearing mice with novel CAIX-specific (ureido)-sulfonamide and glycosylcoumarins inhibitors resulted in a significant inhibition of tumor growth and metastasis formation (1).

These reports emphasize that CAIX specific sulfonamides are promising to pursue for their tumor-specific therapeutic properties with irradiation. The aim of this study was to explore in genetic models the antitumor activity of an indanesulfonamide (11c) with high affinity for CAIX, compared with the general clinically approved CA inhibitor acetazolamide (AZA). First we investigated the binding property of a fluorescent labeled sulfamate (FC11) to validate the genetic models. Furthermore, we hypothesize that specific inhibition of CAIX activity only targets CAIX under hypoxia resulting in decreased tumor cell proliferation, induced of apoptosis and an enhanced effect of irradiation in vivo in a CAIX dependent manner. 


\section{Material and Methods}

\section{Cell culture and model}

Exponentially growing colorectal (HT-29, ATCC HTB-38) carcinoma cells were cultured in Dulbecco's modified Eagle's medium supplemented with $10 \%$ fetal bovine serum. To knockdown CA9, the HuSH-29 shRNA targeting CA9 (TR314250) and empty vector (R20003) were purchased from Origene. Cell lines were transfected using FuGENE 6 (Roche, Germany) according to manufacturer's instructions. Cells were then grown under selective pressure $(300 \mathrm{ng} / \mathrm{ml}$ puromycin) until no mock-transfected cells remained. Two individual clones were selected using cloning cylinders (Sigma) and maintained under selection. Data of the independent clone KD2 are presented in supplementary figure 6.1. Low oxygen conditions were acquired in a MACS VA500 micro-aerophilic workstation (Don Whitley Scientific, Shipley, UK). The atmosphere in the chamber consisted of $0.2 \%$ (hypoxia) or $<0.02 \%$ (anoxia, $0 \%$ ) $\mathrm{O}_{2}, 5 \% \mathrm{CO}_{2}$ and residual $\mathrm{N}_{2}(11)$. In parallel, normoxic $\left(20 \% \mathrm{O}_{2}\right)$ dishes were incubated in air with $5 \%$ $\mathrm{CO}_{2}$.

\section{Inhibition CAIX activity}

The fluorescent labeled sulfamate (FC11) directed against CAIX has been prepared by reaction of sulfamyl chloride with the residue obtained from the reaction of fluorescein isothiocyanate (FITC) with 4-aminophenol (see supplementary data). 11c was synthesized as previously described (12) and acetazolamide (AZA) was obtained from Sigma. The structure and Ki value of the different compounds are depicted in figure 6.1A. Compounds were dissolved in culture medium containing $10 \%$ DMSO at a final concentration of $100 \mu \mathrm{M}$ just before addition to the cells. For the animal experiments, $11 \mathrm{c}$ and AZA were dissolved in $\mathrm{NaCl} 0.9 \%$ containing $1 \%$ DMSO to a final concentration of $45 \mathrm{mg} / \mathrm{kg}$ and injected intravenously via a lateral tail vein.

\section{Immunoblotting and quantitative real-time PCR}

Experiments were performed as previously described $(8,13)$. Antibodies used were M75 (kindly provided by Silvia Pastorekova, Institute of Virology, Slovak Academy of Science, Bratislava, Slovak Republic) against CAIX or $\beta$-actin (Cell Signaling) as loading control. CAIX (F-CATCCTAGCCCTGGTTTTTGG, R-GCTCACACCCCCTTTGGTT) and VEGF (FGACTCCGGCGGAAGCAT, R- TCCGGGCTCGGTGATTTA) gene abundance was detected with power SYBR ${ }^{\circ}$ Green I (Applied Biosystems) and normalized to the expression levels of 18 S RNA. 


\section{FACS analysis}

Cells were incubated with FC11 30 minutes before fixation in freshly prepared 2\% paraformaldehyde. Apoptosis was detected using Alexa Fluor ${ }^{\circledR} 488$ annexin V (Molecular Probes) and propidium iodide (PI; Sigma) according to the manufacturer's guidelines. Fluorescence was analyzed using flow cytometry (FacsSort, BD Biosciences) with FITC and TRITC settings and data were normalized to the signal intensity of normoxic exposed cells. Apoptotic cells were scored as annexin $\mathrm{V}$ positive and $\mathrm{PI}$ negative.

\section{Immunofluorescence}

Cells were grown in culture slides (BD Biosciences), incubated with FC11, fixed with $2 \%$ paraformaldehyde and fluorescence was visualized using a Zeiss Axioskop. Cells for apoptosis staining were permeabilized using 0.2\% Triton X-100 (Sigma). Non-specific binding was blocked using $5 \%$ normal goat serum (RT, 2 h). Cells were incubated (4ㅇ, ON) with cleaved Caspase-3 monoclonal antibody (Cell Signaling) followed by incubation (RT, 2 h) goat anti-rabbit Alexa Fluor ${ }^{\circledR} 488$ conjugate. Before mounting with Shandon Immunomount (Thermo Fisher), cells were incubated with DAPI (RT, $10 \mathrm{~min}$ ).

\section{CA activity assay}

Carbonic anhydrase activity was determined as described previously (14). Cells were washed with cold isotonic HBS buffer and incubated (20 min) with sulfonamide. $\mathrm{pH}$ was monitored over time before and after addition of cold $\mathrm{CO}_{2}$ saturated solution.

\section{Lactic acid assay}

Lactic acid concentration in the culture medium of cells exposed to sulfonamide treatment was determined using the Lactic Acid kit (Biosentec) following manufacturer's instructions.

\section{Animal experiments}

Cells were resuspended in Basement Membrane Matrix (Matrigel ${ }^{\mathrm{TM}}$ BD Biosciences) and injected subcutaneously into the lateral flank of adult NMRI-nu (nu/nu) mice (28$32 \mathrm{~g})$. Animals were treated with sulfonamides at a tumor volume of $165 \pm 58 \mathrm{~mm}^{3}$ for 5 days ( $45 \mathrm{mg} / \mathrm{kg}$ daily) and irradiated with a single dose (10 Gy, (15)) in the middle of the treatment. Tumor growth was monitored until reaching $4 x$ the volume at irradiation time and treatment toxicity was scored by body weight measurements. 
Animal facilities and experiments were in accordance with local institutional guidelines for animal welfare and were approved by the Animal Ethical Committee of the University.

\section{Cell growth}

Cells were seeded at 5000 cells/well in 24-well plates and exposed for normoxia or hypoxia for 1-3 days. After incubation, cells were fixed with $4 \%$ paraformaldehyde (10 $\mathrm{min}$ ) and stained with $0.1 \%$ Crystal violet solution (30 $\mathrm{min}$ ). Extraction was done with $10 \%$ Acetic Acid (15 min) and absorbance at $590 \mathrm{~nm}$ was measured (FLUOstar Omega).

\section{Clonogenic survival}

Cells were exposed to normoxia or anoxia for $24 \mathrm{~h}$, subsequently irradiated ( $N: 2,4,6$ and 8 Gy; A: 4, 8, 12 and 16 Gy; 225 kV Philips X-ray tube), trypsinized and plated in triplicate for clonogenic survival. Cells were allowed to form colonies during 14 days which were fixed and stained with $4 \%$ methylene blue in $70 \%$ ethanol. Afterwards, colonies were counted in an automated way (Oxford Optronix).

\section{Statistics}

All statistical analyses were performed with GraphPad Prism version 5.01 for Windows (GraphPad Software, 2007, California, USA). An unpaired student's t-test and nonparametric Mann-Whitney $U$ test for small groups were used to determine the statistical significance of differences between two independent groups of variables. For all tests, a $\mathrm{P}<0.05$ was considered significant.

\section{Results}

HT-29 colorectal carcinoma cells showed elevated CA9 mRNA and CAIX protein levels in response to reduced oxygen concentrations, which remained high upon reoxygenation (figure $6.1 \mathrm{~B}$ and $\mathrm{C}$ ). To investigate the role of CAIX in extracellular acidosis and to facilitate the establishment of a causal relationship between CAIX expression and its therapeutic effect in more detail, CA9 was silenced genetically in a constitutive manner. A significant reduction in CA9 mRNA levels was observed for the $\mathrm{KD}$ cell line at $0.2 \%$ (hypoxia; $\mathrm{P}=0.002$ ) and $0 \%$ (anoxia; $\mathrm{P}=0.029$ ) $\mathrm{O}_{2}$ as compared with a scrambled control (EV) cell line, which demonstrated similar expression levels as the HT-29 parental cell line. Additionally, CAIX protein levels were abolished in the KD 
cells (figure 6.1C). Furthermore, other HIF responsive genes were not affected as demonstrated by the increased VEGF expression levels upon reduced oxygen concentrations for both cell lines (figure 6.1B).

A
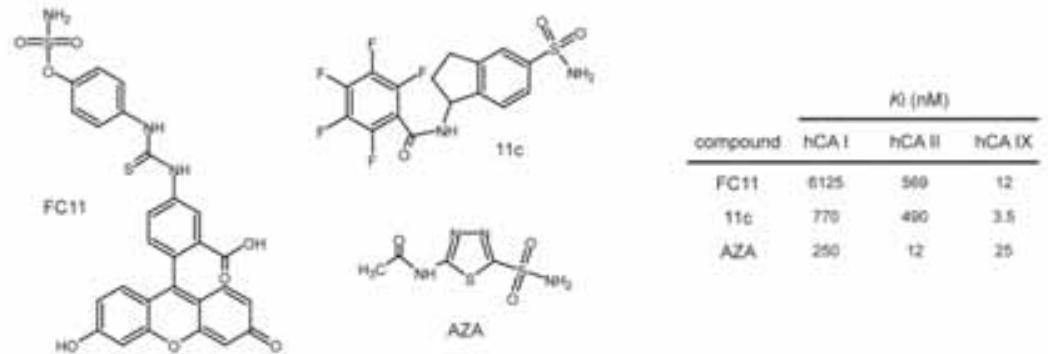

B
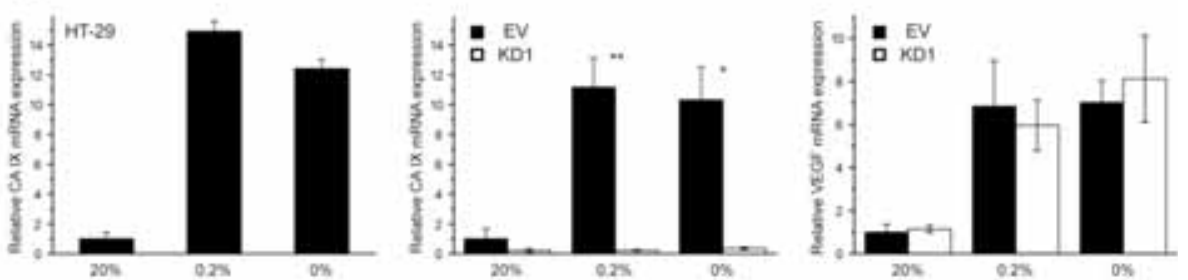

C

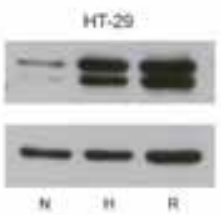

EV

KD1
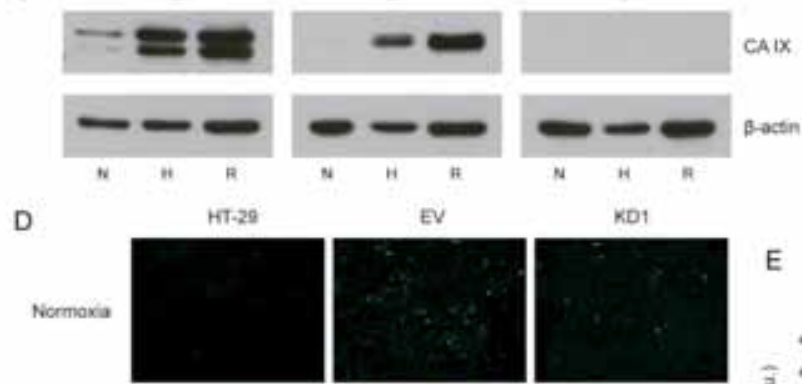

EV
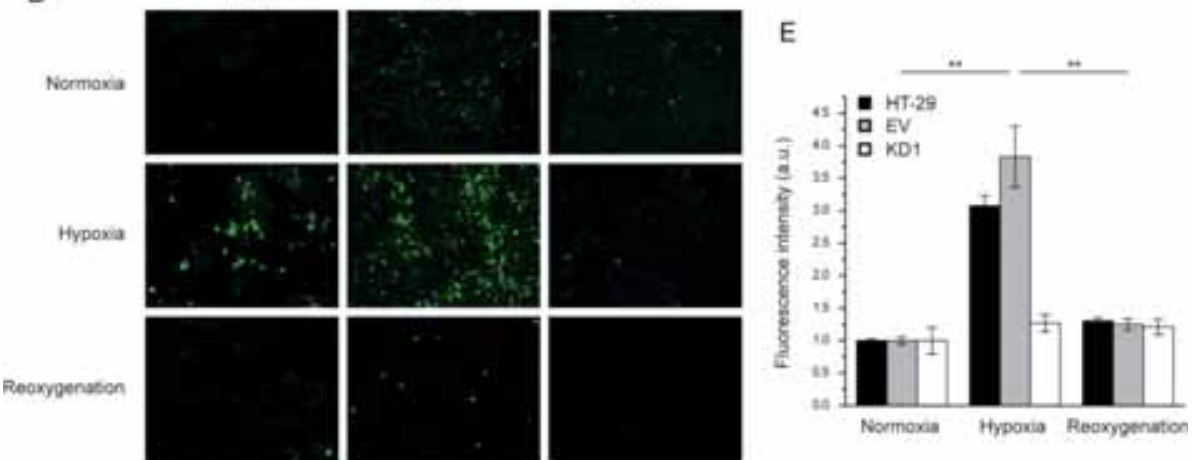

Figure 6.1 (A) Chemical structure and affinity (Ki) for CAI, CAll and CAIX of the investigated CA inhibitors. (B) CAIX and VEGF mRNA expression under different oxygen concentrations (\%) relative to normoxia for HT-29 parental, EV and KD1 cells. (C) Western blot analysis of CAIX expression in normoxic (N), hypoxic (H) or reoxygenated $\left(\mathrm{R}: 0.2 \% \mathrm{O}_{2}, 24 \mathrm{~h}\right.$ followed by $1 \mathrm{~h} 20 \% \mathrm{O}_{2}$ ) conditions for the different cell lines. $\beta$-Actin was used as loading control. (D) Representative fluorescence analysis of cells treated with $100 \mu \mathrm{M}$ FC11 under the respective oxygen conditions. (E) Quantitative FACS analysis of FC11 binding relative to normoxia. Data represent the mean $\pm S D$ of at least three independent experiments. Asterisk indicates significant difference $\left({ }^{* P}<0.05,{ }^{*} \mathrm{P}<0.01\right)$. Data for an independent CAIX KD (KD2) are shown in supplementary figure 6.1 
A
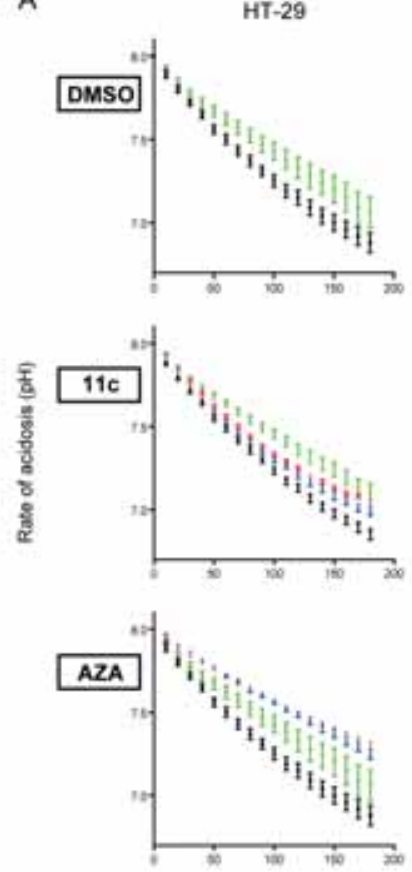

EV
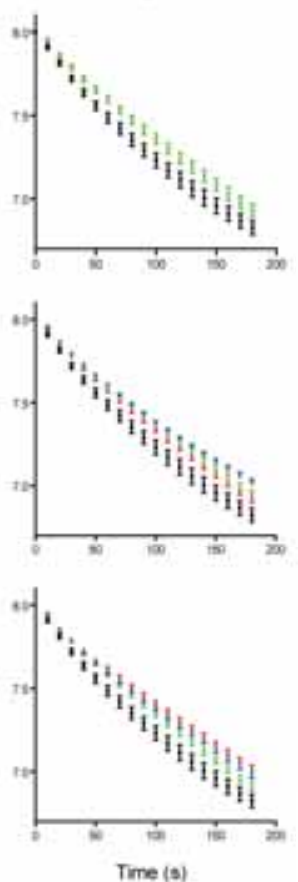

KD1
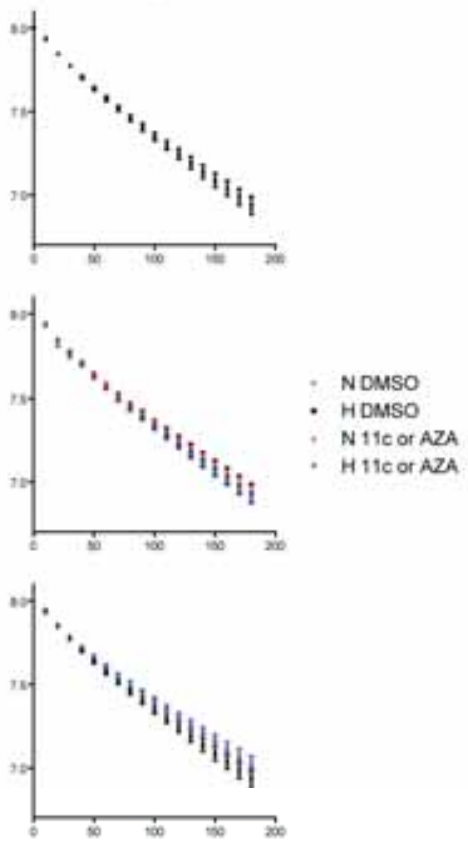

B

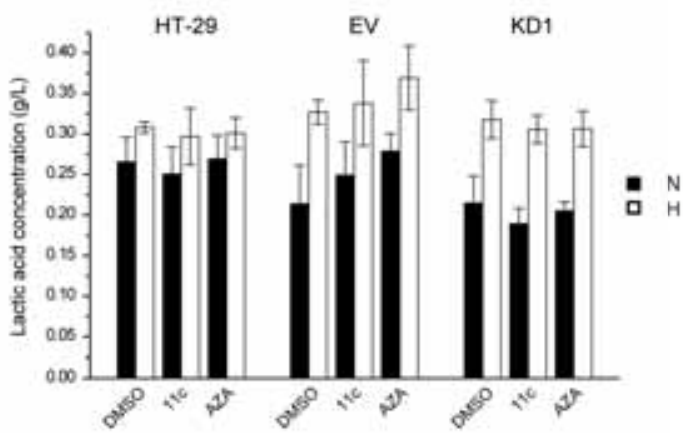

Figure 6.2 (A) Extracellular rate of acidification and (B) medium lactic acid concentrations of cells exposed to normoxia (N) or hypoxia (H) after pre-treatment with $100 \mu \mathrm{M} 11 \mathrm{c}$, AZA or vehicle (DMSO). Data represent the mean \pm SD of at least four independent experiments. Data for an independent CAIX KD (KD2) are shown in supplementary figure 6.1 . 
To define the oxygen conditions required for inhibitor binding, accumulation of the fluorescent sulfamate FC11 with high affinity for CAIX ( $\mathrm{Ki}=12 \mathrm{nM})$ was investigated. Significant higher $(\mathrm{P}=0.009) \mathrm{FC11}$ binding was observed at HT-29 and EV cells exposed to hypoxia $(0.2 \%)$ as compared with their normoxic counterparts (figure 6.1D and E), corresponding with elevated CAIX expression. Binding was dramatically reduced $(P=$ 0.002) compared with hypoxic conditions when FC11 was added upon reoxygenation, although CAIX expression levels remained high. No FC11 accumulation was observed at KD cells, corresponding with the absence of CAIX expression.

Next we examined whether CAIX depletion resulted in a decreased capacity to acidify the extracellular environment upon hypoxia. The faster rate of acidification upon hypoxia was abolished in the KD cells relative to the HT-29 parental and EV cells (figure 6.2A top). To define the necessity for CAIX activity for extracellular acidification in hypoxic conditions, the effect of a specific CAIX (11c) and a general CA (AZA) sulfonamide inhibitor on hypoxia-induced changes in extracellular $\mathrm{pH}$ was tested in these cells. The hypoxic acidification rate could be inhibited by addition of $11 \mathrm{c}$ to HT29 and EV cells, while no effect was seen in the KD cells (figure 6.2A middle). The effect of AZA was even more pronounced, resulting in an acidification rate lower then seen under normoxic conditions (figure $6.2 \mathrm{~A}$ bottom) and was also observed for the KD cells, indicating the lack of specificity of AZA for CAIX. Production of lactic acid was significantly higher upon hypoxia and no differences could be observed in cultures of CAIX-positive and CAIX-negative cells or upon CAIX activity inhibition (figure 6.2B), indicating that the lower hypoxic rate of acidification upon CAIX activity inhibition was not caused by interfering with the lactic acid production pathway.

To investigate in this exploratory study the consequences of CAIX activity inhibition on tumor growth and radiotherapy response, HT-29 tumors were established and mice were treated with $11 \mathrm{c}$ at a volume of $165 \mathrm{~mm}^{3}$ for 5 days and irradiated with a single dose (10 Gy) at the middle of the treatment. 11c treatment resulted in a significant slower tumor growth $(P=0.024)$ compared to vehicle controls. An increased specific doubling time was observed when treated with irradiation as a single treatment, which was further increased $(P=0.016)$ by combining $11 \mathrm{c}$ with irradiation (figure 6.3A top). To exclude non-specific activity of $11 \mathrm{c}$, experiments were repeated in the EV and KD models. A first observation was that the KD tumor grew slower compared with the EV tumor, indicating the importance of CAIX expression for tumor progression. Therefore, animals were treated with sulfonamides at a similar volume for both the EV and the KD tumors. No effect of $11 \mathrm{c}(\mathrm{P}=0.530)$ and no enhanced tumor effect upon combination 
A
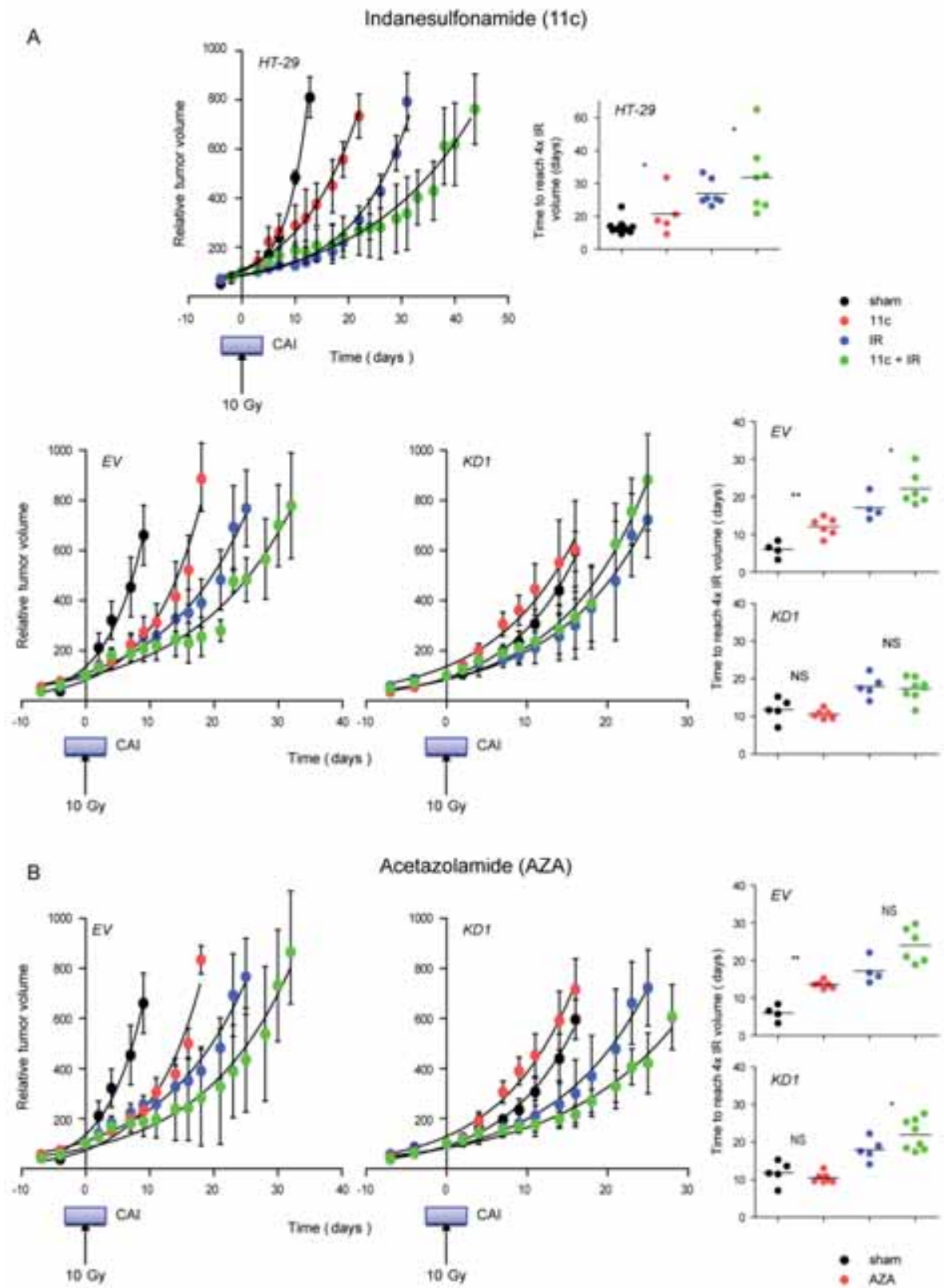

Acetazolamide (AZA)
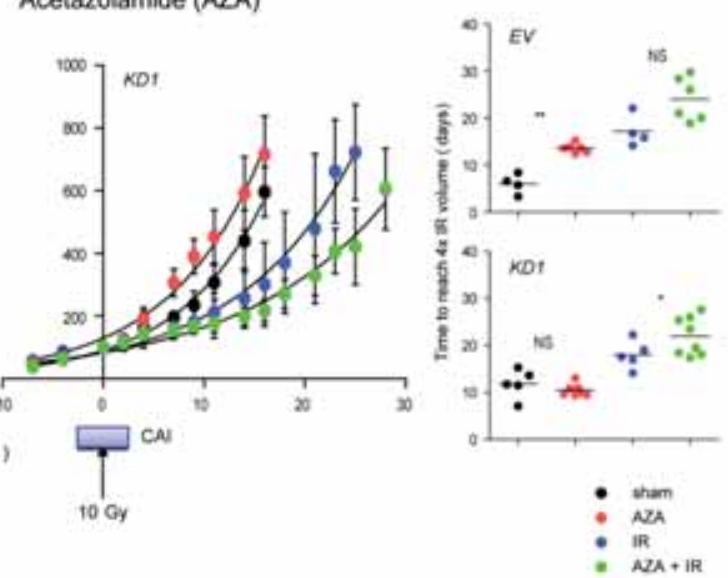

Figure $6.311 \mathrm{c}(\mathrm{A})$ or AZA (B) was administered when tumors reached an average size of $165 \mathrm{~mm} 3(45 \mathrm{mg} / \mathrm{kg}$, 5 days) and tumors were irradiated (10 Gy) at day 3 (set to 0 ) of this treatment. Tumor growth was monitored until reaching $4 x$ the volume at irradiation time and specific doubling time was calculated. Data represent the mean \pm SD of four to eight independent animals and asterisks indicate statistical significance $(* P<0.01 ; * * P<0.001), N S=$ not significant. 

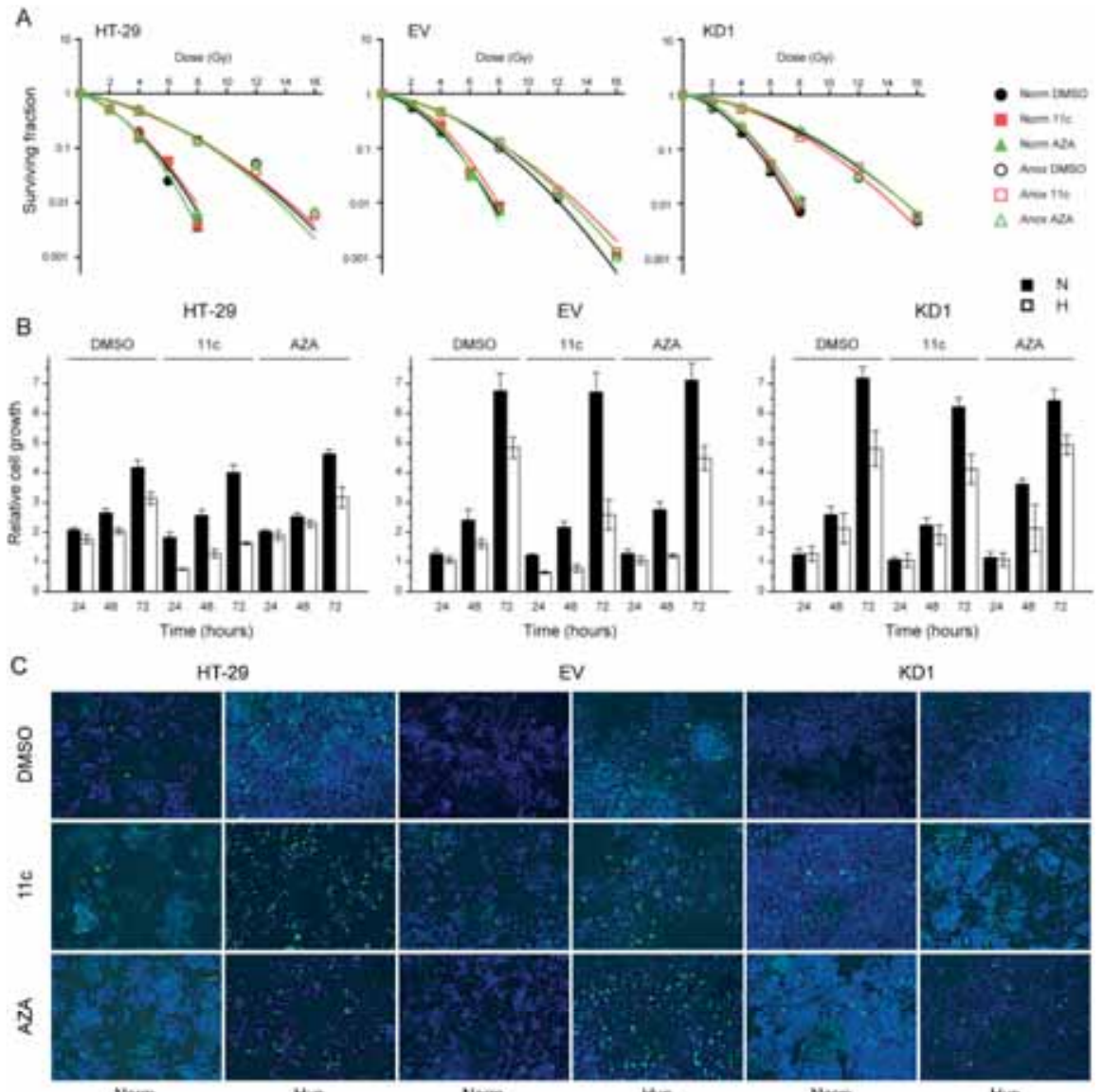

HT-29

EV

KD1
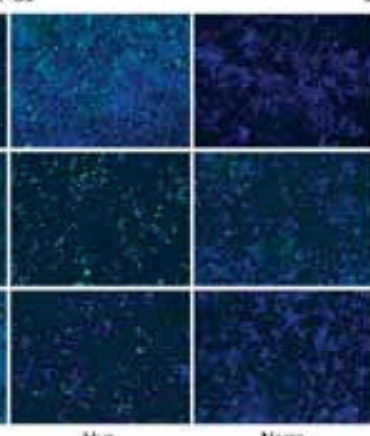

ev

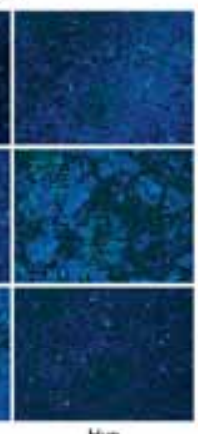

D

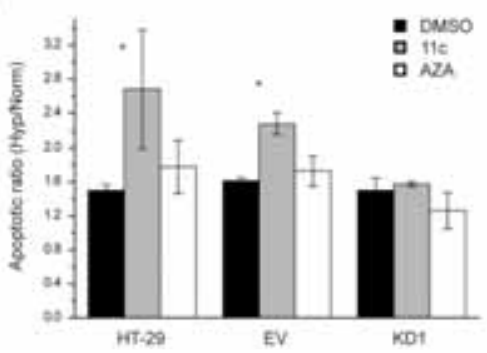

Figure 6.4 (A) Clonogenic survival assay after exposure to different doses of irradiation upon normoxia (Norm: 0, 2, 4, 6 and $8 \mathrm{~Gy}$ ) and anoxia (Anox: 0, 4, 8, 12 and $16 \mathrm{~Gy}$ ) exposed cells after pre-treatment with $100 \mu \mathrm{M} \mathrm{11c}$ or AZA. (B) Cell proliferation of cells exposed to $100 \mu \mathrm{M} \mathrm{11c}$ or AZA was monitored under normoxic (N) or hypoxic $(\mathrm{H})$ conditions by Crystal Violet staining after 1-3 days of growth. (C) Representative images of cleaved Caspase-3 staining (green) of normoxic (Norm) or hypoxic (Hyp) cells exposed to $100 \mu \mathrm{M}$ 11c or AZA. Nuclear counterstaining is assessed with DAPI (blue). (D) Quantitative FACS analysis of the hypoxia to normoxia (Hyp/Norm) apoptotic ratio, as assessed by Alexa Fluor ${ }^{\circledR}$ annexin $V$ fluorescence. Data represent the mean $\pm S D$ of three independent experiments. Asterisk indicates significant difference $(* P<$ 0.05). Data for an independent CAIX KD (KD2) are shown in supplementary figure 6.1. 
treatment $(P=0.724)$ were observed relative to the vehicle control and irradiation alone, respectively for the KD tumors as compared with the EV tumor model (figure 6.3A bottom), demonstrating the CAIX specificity of 11c treatment. Next, we examined if the clinically approved general CA inhibitor AZA exerted similar effects on therapy response. AZA treatment resulted in an increased specific doubling time only in the EV group ( $P=0.009$ ). Although no effect was found for the KD tumors ( $P=0.202), A Z A$ demonstrated additional effects on both groups when combined with irradiation (figure 6.3B), confirming its broader spectrum of action. Additionally, none of the treatment schedules caused any observable toxicity as monitored by body weight loss (data not shown).

To exclude that CA inhibition has an effect on intrinsic radiosensitivity, the response of the EV and KD cells to a combination of sulfonamides and a range of radiation doses in vitro was measured under normoxia and anoxia (figure 6.4A). Both cell lines demonstrated a more radioresistant phenotype under anoxic conditions. However, incubation with $11 \mathrm{c}$ or AZA before irradiation did not result in enhancement of the irradiation effect. Therefore the increased growth delay could not be explained by changes in radiosensitivity.

Next we investigated whether inhibition of CAIX activity could affect proliferation and apoptosis as possible explanation for the in vivo enhanced effect when combining irradiation and sulfonamides. In general, proliferation was higher for the EV and KD cells compared with the HT-29 parental cell line. Under normoxic conditions, neither 11c nor AZA inhibited tumor cell proliferation (figure 6.4B). Although cell growth decreased for all cell lines under hypoxic conditions, proliferation was more reduced by $11 \mathrm{c}$ and AZA administration only in CAIX expressing cells. However, the antiproliferative capacity of AZA was lower than that of $11 \mathrm{c}$. In the KD cells, the sulfonamides could not decrease cell proliferation (figure 6.4B). AZA and more efficiently 11c significantly induced early apoptotic events in HT-29 parental and EV cells under hypoxia as monitored by cleaved caspase-3 fluorescence (figure 6.4C), while no effect was found for the KD cells. Flow cytometry detection of annexin $V$ showed an increased hypoxia to normoxia apoptotic ratio for $11 \mathrm{c}$ and to a lesser extent for AZA only in HT-29 and EV cells (figure 6.4D), confirming our findings on caspase-3 activation. 


\section{Discussion}

CAIX has been associated with tumorigenic transformation, tumor cell migration and invasion through its capacity to acidify the extracellular microenvironment of hypoxic tumors (16) and is therefore a potential therapeutic target. Recently, sulfonamidebased CAIX inhibitors have been proposed as potential antitumor agents (6). Accumulation of a novel fluorescent labeled sulfamate FC11 (Ki = $12 \mathrm{nM})$ correlated with CAIX expression in HT-29 and EV cells exposed to hypoxia. Although CAIX expression levels remained high upon reoxygenation in these cells, in agreement with the known half-life of $38 \mathrm{~h}$ (17), sulfamate accumulation was markedly reduced. Additionally, no accumulation was observed in CAIX KD cells. Previously, we obtained similar results with a fluorescent labeled sulfonamide $(7,8)$ and together these data indicate that sulfonamides only accumulate on CAIX expressing cells during hypoxia.

Hypoxia is known to cause an acidification of the extracellular environment and it has been reported that CAIX is the main contributor for this process $(4,14)$. We obtained similar data showing that CAIX depletion slows the faster acidification rate under hypoxia. Furthermore, acetazolamide and indanesulfonamide $11 \mathrm{c}$ were also able to prevent extracellular acidification only during hypoxia in a CAIX-dependent manner, with a more CAIX-specific effect for 11c compared to the general CA inhibitor acetazolamide, in agreement with previous reports (14). It has been reported that lactic acid production is increased upon hypoxia and could predict treatment outcome by its possible contribution to the tumor acidification (18). Our data indicate that CAIX inhibition had no influence on lactic acid production either under normoxia or hypoxia. Similar results were obtained upon elimination of the CAIX catalytic active domain (4), indicating that the extracellular acidity upon hypoxia better is explained by CAIX activation than by lactic acid production.

Recently, it has been demonstrated that the tumor response to fractionated irradiation is determined by the amount of extracellular acid production $(18,19)$. Since CAIXspecific sulfonamides counteract extracellular acidification, we hypothesize that these compounds are able to sensitize tumor to irradiation. First, we observed that CAIX silencing resulted in slower tumor growth relative to the scrambled controls. Previous reports from breast and colorectal xenograft models confirmed the requirement of hypoxia-induced CAIX expression in the regulation of tumor growth $(1,20)$. Single treatment with CAIX-specific sulfonamide 11c or general CAIX inhibitor acetazolamide resulted in a significant slower tumor growth compared to vehicle controls in a CAIXdependent manner. Similar results were obtained using CAIX-specific (ureido)- 
sulfonamides and glycosylcoumarins or membrane-impermeable derivatives of acetazolamide in mammary or colorectal tumor-bearing mice $(1,9)$. Irradiation as a single treatment increased the specific doubling time independent of CAIX expression. Previously, we have demonstrated that a single dose of $10 \mathrm{~Gy}$ is sufficient to induce a growth delay in colorectal xenografts (21). This is the first report showing that CAIXspecific sulfonamides could increase the effect of irradiation in a CAIX dependent manner, since an effect was observed only for the HT-29 parental and EV group, while no enhancement was observed for the KD tumors, demonstrating the CAIX specificity of the therapy. The reduced hypoxic rate of extracellular acidification upon sulfonamide treatment might explain this increased response to irradiation. In contrast, AZA demonstrated an additional effect in both groups when combined with irradiation, which could be explained by the additional inhibition of the intracellular CAll. Recently, it has been shown that CAll is critical for $\mathrm{pH}$ homeostasis in Gl-tract organs (22) and therefore treatment of these tissue-related tumors warrants precautions for additional toxicity.

We also examined the possible molecular mechanisms underlying this antitumor effect of CAIX inhibition. The increased growth delay of the combination treatment could not be explained by changes in intrinsic radiosensitivity, since the compounds had no effect on survival of cells exposed in vitro to a range of radiation doses. The current exploratory study suggests that the enhanced effect of sulfonamide treatment combined with irradiation is the result of changes in the tumor microenvironment which renders it more sensitive to irradiation. On the other hand, in vitro inhibition of CAIX activity using sulfonamides also decreased cell proliferation and induced apoptosis only upon hypoxia and could therefore induce a significant antitumor effect, in agreement with previous reports (10). Maintaining a neutral intracellular $\mathrm{pH}$ is a key cellular strategy to protect tumor cells against apoptotic death and to permit tumor cell proliferation. Our data suggest that the potential antitumor effect of CAIX inhibition may be linked to intracellular acidosis and the underlying molecular mechanisms are currently under investigation. Furthermore, the decreased cell proliferation and induced apoptosis was more efficiency when inhibition is selective suggesting that the antitumor effect of CA inhibitors is mainly mediated by inhibition of CAIX. Furthermore, it has been shown that general CA inhibition using AZA can inhibit the invasive capacity of cells and this effect is attributed to inhibition of the CAll and CAXII isoforms, since only a low response was observed for CAIX-positive cells (23). 
To exclude that the therapeutic effect of the combination of sulfonamide and irradiation results from normal tissue effects, it is necessary to investigate radiationinduced damage on intestinal (short-term toxicity) and lung (long-term toxicity) epithelium and to calculate the dose modifying factors (DMF) for both tumor and normal tissue. Recently, experts of an ESTRO committee aiming at writing guidelines for the development of targeted drugs associated with radiation, concluded that such normal tissue experiments are needed before new agents are tested with radiotherapy in a clinical setting (D. Hollywood, personal communication) (24). We expect to find only an increased DMF for the tumor, since no signs for normal tissue toxicity were observed in the current study. Furthermore, we are currently designing stable carcinoma cells which can be induced to down-regulate gene expression in order to closely mimic the clinical situation, since tumors should be established with a wild type genotype and subsequently the CAIX gene can be switched off, allowing to investigate the effect of CAIX down-regulation.

In conclusion, our experiments demonstrate that specific inhibition of CAIX activity only targets CAIX under hypoxia resulting in a decreased hypoxic rate of extracellular acidification and tumor cell proliferation and induction of apoptosis without affecting the intrinsic radiosensitivity. In vivo, a therapeutic enhancement was observed only in CAIX expressing tumors when combined with irradiation, indicating a causal relationship between CAIX expression and its therapeutic effect. Furthermore, our data indicate the potential utility of CAIX inhibition as a new strategy for tumor-specific targeting to eventually improve the response to irradiation treatment.

\section{Acknowledgements}

This work has been funded with the support of the EU $6^{\text {th }}$ framework Program (Euroxy project ref. 2003-502932), the EU $7^{\text {th }}$ framework (Metoxia project ref. 2008-222741) and is supported by KWF and the Oxford Research UK, Oxford Cancer Imaging Centre. 


\section{Corrigendum for:}

Specific inhibition of carbonic anhydrase IX activity enhances the in vivo therapeutic effect of tumor irradiation. j.radonc.2011.05.045 (Dubois et al., 2011)

In this article (Volume 99, Issue 3, Pages 424-431) published in the June 2011 issue of Radiotherapy and Oncology, we reported the use HT-29 EV (empty vector) and shCA9 (Carbonic anhydrase IX targeting shRNA) cell lines. Using Short tandem repeat (STR) analysis we recently discovered that these cell lines are of HCT116 origin instead another colon cancer cell line. Since the proper empty vector control CAIX expressing cell line was included in the paired cell lines for both publications, our conclusion stating that the therapeutic efficacy of blocking CAIX activity is causally dependent on CAIX expression is still valid. The authors regret this error. 


\section{Supplementary data}

Synthesis of 2-(3-hydroxy-6-oxo-6H-xanthen-9-yl)-5-(3-(4-hydroxyphenyl)thioureido)benzoic acid (compound 1) (25).

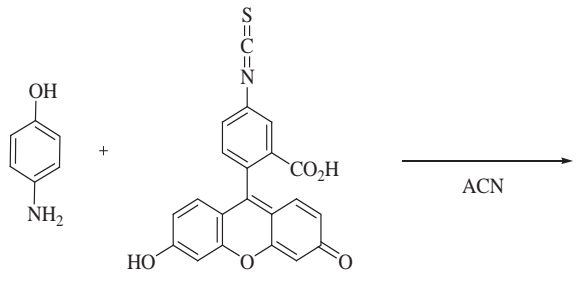

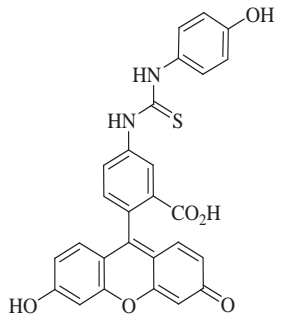

19

4-Aminophenol $(0.1 \mathrm{~g}, 1.0 \mathrm{eq})$ was added to a suspension of fluorescein isothiocyanate $(0.36 \mathrm{~g}, 1.0 \mathrm{eq})$ in dry $\mathrm{ACN}(10 \mathrm{ml})$ and the reaction was stirred under a nitrogen atmosphere overnight. The solids were separated by filtration and the filtrate concentrated under vacuum. The residue was purified by silica gel column chromatography eluting with ethyl acetate to afford 1 as an orange solid in $57 \%$ yield.

Synthesis of 2-(3-hydroxy-6-oxo-6H-xanthen-9-yl)-5-(3-(4-(sulfamoyloxy)phenyl)thioureido) benzoic acid (compound 2 = FC11).<smiles>O=C(O)c1cc(NC(=S)Nc2ccc(O)cc2)ccc1-c1c2ccc(=O)cc-2oc2cc(O)ccc12</smiles>

19<smiles>NS(=O)(=O)c1ccc(NC(=S)Nc2ccc(-c3c4ccc(=O)cc-4oc4cc(O)ccc34)c(C(=O)O)c2)cc1</smiles>

20

Compound 1 (0.1g, $1.0 \mathrm{eq})$ was dissolved in dry DMA $(3.0 \mathrm{ml})$ and freshly prepared sulfamyl chloride $(10 \mathrm{~g}, 1.0 \mathrm{eq})$ was added at room temperature portion-wise. The reaction mixture was stirred at room temperature for 5 minutes, quenched with slush and the solid formed was filtrated under vacuum, dried on air and finally purified by silica gel column chromatography eluting with ethyl acetate to afford compound 2 (FC11) as an orange solid in $56 \%$ yield. 


\section{Characterization}

(1): silica gel TLC $R_{f} 0.11$ (Ethyl Acetate); $\delta_{H}\left(400 \mathrm{MHz}, \mathrm{DMSO}-\mathrm{d}_{6}\right) 6.49\left(1 \mathrm{H}, \mathrm{dd}, \mathrm{J} 7.5,6^{\prime}\right.$ $\mathrm{H}), 6.65(4 \mathrm{H}, \mathrm{brs}), 6.70(2 \mathrm{H}, \mathrm{s}), 6.80(2 \mathrm{H}, \mathrm{d}, \mathrm{J} 8.8), 7.22(2 \mathrm{H}, \mathrm{d}, \mathrm{J} 8.8), 8.83(1 \mathrm{H}, \mathrm{d}, \mathrm{J} 7.5$, $\left.5^{\prime}-\mathrm{H}\right), 8.18\left(1 \mathrm{H}, \mathrm{s}, 2^{\prime}-\mathrm{H}\right), 9.49(1 \mathrm{H}, \mathrm{s}$, exchange with $\mathrm{D} 2 \mathrm{O}, 1-\mathrm{OH}), 9.87(1 \mathrm{H}, \mathrm{s}$, exchange with $\mathrm{D} 2 \mathrm{O}, \mathrm{NH}), 9.95(1 \mathrm{H}, \mathrm{s}$, exchange with $\mathrm{D} 2 \mathrm{O}, \mathrm{NH}), 10.18(1 \mathrm{H}, \mathrm{s}$, exchange with $\mathrm{D} 2 \mathrm{O}$, $\mathrm{OH}) ; \delta_{\mathrm{C}}\left(100 \mathrm{MHz}, \mathrm{DMSO}-\mathrm{d}_{6}\right) 180.9$ (C=S), 169.6, 160.5, 156.2, 152.9, 148.6, 142.5, $131.9,131.2$, 131.0, 130.1, 127.4, 127.38, 125.0, 118.7, 116.7, 116.6, 116.2, 113.7, $110.7,103.3,84.1$

(2 = FC11): silica gel TLC $R_{f} 0.07$ (Ethyl acetate); $\delta_{H}\left(400 \mathrm{MHz}\right.$, DMSO- $\left.\mathrm{d}_{6}\right)$ 7.06-7.38 $(8 \mathrm{H}$, m), $7.40(2 \mathrm{H}, \mathrm{s}), 7.62(2 \mathrm{H}, \mathrm{d}, \mathrm{J} 8.8), 7.93(1 \mathrm{H}, \mathrm{d}, \mathrm{J} 7.5), 8.10(2 \mathrm{H}, \mathrm{s}$, exchange with D2O, $\left.\mathrm{SO}_{2} \mathrm{NH}_{2}\right), 8.26(4 \mathrm{H}, \mathrm{s}), 8.32(1 \mathrm{H}, \mathrm{s}), 10.20(1 \mathrm{H}, \mathrm{s}$, exchange with $\mathrm{D} 2 \mathrm{O}, \mathrm{NH}), 10.31(1 \mathrm{H}, \mathrm{s}$, exchange with $\mathrm{D} 2 \mathrm{O}, \mathrm{NH}) ; \delta_{\mathrm{C}}\left(100 \mathrm{MHz}, \mathrm{DMSO}-\mathrm{d}_{6}\right) 180.8(\mathrm{C}=\mathrm{S}), 169.1,162.0,152.5$, $151.7,148.3,147.8,145.8,142.7,138.9,138.3,131.8,130.5,127.5,126.6,126.1$, $125.0,123.7,123.3,119.8,119.7,118.8,117.9,111.5,81.6$. 
A

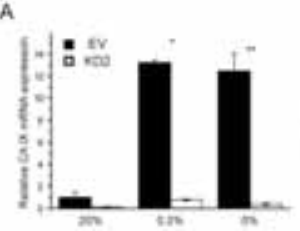

C

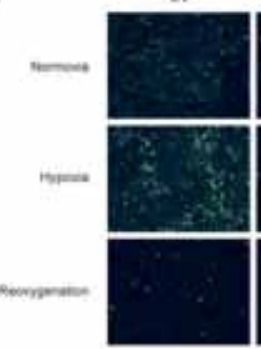

E

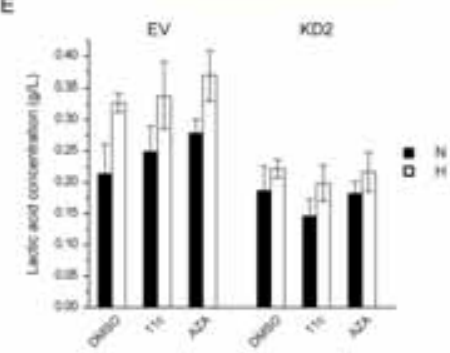

G

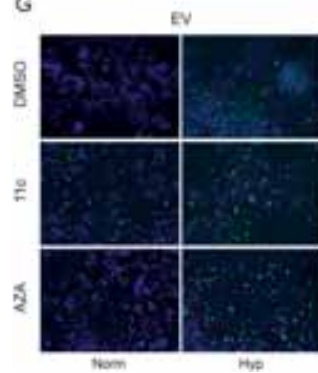

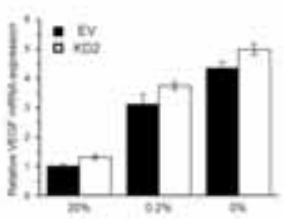

B

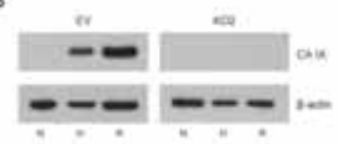

D

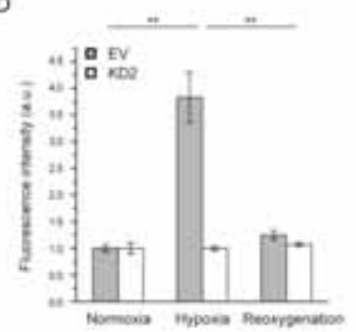

F

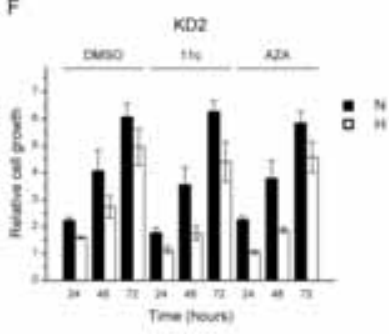

H
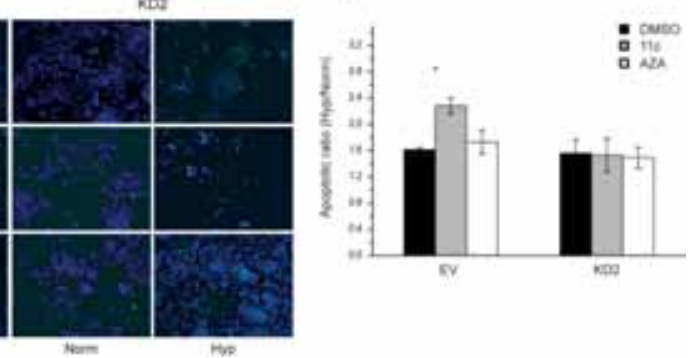

Supplementary figure 6.1 (A) CAIX and VEGF mRNA expression under different oxygen concentrations relative to normoxia for EV and KD2 cells. (B) Western blot analysis of CAIX expression in normoxic (N), hypoxic $(\mathrm{H})$ or reoxygenated $\left(\mathrm{R}: 0.2 \% \mathrm{O}_{2}, 24 \mathrm{~h}\right.$ followed by $\left.1 \mathrm{~h} 20 \% \mathrm{O}_{2}\right)$ conditions for the different cell lines. $\beta$ Actin was used as loading control. (C) Representative fluorescence analysis of cells treated with $100 \mu \mathrm{M}$ FC11 under the respective oxygen conditions. (D) Quantitative FACS analysis of FC11 binding relative to normoxia for EV and KD2 cells. (E) Medium lactic acid concentrations of cells exposed to normoxia (N) or hypoxia (H) after pre-treatment with $100 \mu \mathrm{M}$ 11c or AZA. (F) Cell proliferation of EV and KD2 cells exposed to $100 \mu \mathrm{M}$ $11 \mathrm{c}$ or AZA was monitored under normoxic $(\mathrm{N})$ or hypoxic $(\mathrm{H})$ conditions by Crystal Violet staining after 1-3 days of growth. (G) Representative images of cleaved Caspase-3 staining (green) of normoxic (Norm) or hypoxic (Hyp) cells exposed to $100 \mu \mathrm{M} 11 \mathrm{c}$ or AZA. Nuclear counterstaining is assessed with DAPI (blue). (H) Quantitative FACS analysis of the hypoxia to normoxia (Hyp/Norm) apoptotic ratio, as assessed by Alexa Fluor $^{\circledR}$ annexin $V$ fluorescence. Data represent the mean \pm SD of at least three independent experiments. Asterisk indicates significant difference $(* P<0.05, * * P<0.01)$. 


\section{References}

1. Lou Y, McDonald PC, Oloumi A, Chia SK, Ostlund C, Ahmadi A, et al. Targeting Tumor Hypoxia: Suppression of Breast Tumor Growth and Metastasis by Novel Carbonic Anhydrase IX Inhibitors. Cancer Res. 2011.

2. Potter $\mathrm{CP}$, Harris AL. Diagnostic, prognostic and therapeutic implications of carbonic anhydrases in cancer. Br J Cancer. 2003;89:2-7.

3. Alterio V, Hilvo M, Di Fiore A, Supuran CT, Pan P, Parkkila S, et al. Crystal structure of the catalytic domain of the tumor-associated human carbonic anhydrase IX. Proc Natl Acad Sci U S A. 2009;106:16233-8.

4. Svastova E, Hulikova A, Rafajova M, Zat'ovicova M, Gibadulinova A, Casini A, et al. Hypoxia activates the capacity of tumor-associated carbonic anhydrase IX to acidify extracellular pH. FEBS Lett. 2004;577:439-45.

5. Stubbs M, McSheehy PM, Griffiths JR, Bashford CL. Causes and consequences of tumour acidity and implications for treatment. Mol Med Today. 2000;6:15-9.

6. Supuran CT. Carbonic anhydrases: novel therapeutic applications for inhibitors and activators. Nat Rev Drug Discov. 2008;7:168-81.

7. Dubois L, Douma K, Supuran CT, Chiu RK, van Zandvoort MA, Pastorekova S, et al. Imaging the hypoxia surrogate marker CA IX requires expression and catalytic activity for binding fluorescent sulfonamide inhibitors. Radiother Oncol. 2007;83:367-73.

8. Dubois L, Lieuwes NG, Maresca A, Thiry A, Supuran CT, Scozzafava A, et al. Imaging of CA IX with fluorescent labelled sulfonamides distinguishes hypoxic and (re)-oxygenated cells in a xenograft tumour model. Radiother Oncol. 2009;92:423-8.

9. Ahlskog JK, Dumelin CE, Trussel S, Marlind J, Neri D. In vivo targeting of tumor-associated carbonic anhydrases using acetazolamide derivatives. Bioorg Med Chem Lett. 2009;19:4851-6.

10. Cianchi F, Vinci MC, Supuran CT, Peruzzi B, De Giuli P, Fasolis G, et al. Selective inhibition of carbonic anhydrase IX decreases cell proliferation and induces ceramide-mediated apoptosis in human cancer cells. J Pharmacol Exp Ther. 2010;334:710-9.

11. Rouschop KM, Ramaekers CH, Schaaf MB, Keulers TG, Savelkouls KG, Lambin P, et al. Autophagy is required during cycling hypoxia to lower production of reactive oxygen species. Radiother Oncol. 2009;92:411-6.

12. Thiry A, Ledecq M, Cecchi A, Dogne JM, Wouters J, Supuran CT, et al. Indanesulfonamides as carbonic anhydrase inhibitors. Toward structure-based design of selective inhibitors of the tumor-associated isozyme CA IX. J Med Chem. 2006;49:2743-9.

13. Theys J, Jutten B, Dubois L, Rouschop KM, Chiu RK, Li Y, et al. The deletion mutant EGFRvIll significantly contributes to stress resistance typical for the tumour microenvironment. Radiother Oncol. 2009;92:399-404

14. van den Beucken T, Ramaekers CH, Rouschop K, Koritzinsky M, Wouters BG. Deficient carbonic anhydrase 9 expression in UPR-impaired cells is associated with reduced survival in an acidic microenvironment. Radiother Oncol. 2009;92:437-42.

15. Dubois L, Magagnin MG, Cleven AH, Weppler SA, Grenacher B, Landuyt W, et al. Inhibition of 4E-BP1 sensitizes U87 glioblastoma xenograft tumors to irradiation by decreasing hypoxia tolerance. Int J Radiat Oncol Biol Phys. 2009;73:1219-27.

16. Swietach P, Patiar S, Supuran CT, Harris AL, Vaughan-Jones RD. The role of carbonic anhydrase 9 in regulating extracellular and intracellular ph in three-dimensional tumor cell growths. J Biol Chem. 2009;284:20299-310. 
17. Rafajova M, Zatovicova M, Kettmann R, Pastorek J, Pastorekova S. Induction by hypoxia combined with low glucose or low bicarbonate and high posttranslational stability upon reoxygenation contribute to carbonic anhydrase IX expression in cancer cells. Int J Oncol. 2004;24:995-1004.

18. Quennet V, Yaromina A, Zips D, Rosner A, Walenta S, Baumann M, et al. Tumor lactate content predicts for response to fractionated irradiation of human squamous cell carcinomas in nude mice. Radiother Oncol. 2006;81:130-5.

19. Sattler UG, Meyer SS, Quennet V, Hoerner C, Knoerzer H, Fabian C, et al. Glycolytic metabolism and tumour response to fractionated irradiation. Radiother Oncol. 2010;94:102-9.

20. Chiche J, Ilc K, Laferriere J, Trottier E, Dayan F, Mazure NM, et al. Hypoxia-inducible carbonic anhydrase IX and XII promote tumor cell growth by counteracting acidosis through the regulation of the intracellular pH. Cancer Res. 2009;69:358-68.

21. Rouschop KM, van den Beucken T, Dubois L, Niessen H, Bussink J, Savelkouls K, et al. The unfolded protein response protects human tumor cells during hypoxia through regulation of the autophagy genes MAP1LC3B and ATG5. J Clin Invest. 2010;120:127-41.

22. Sjoblom M, Singh AK, Zheng W, Wang J, Tuo BG, Krabbenhoft A, et al. Duodenal acidity "sensing" but not epithelial HCO3- supply is critically dependent on carbonic anhydrase II expression. Proc Natl Acad Sci U S A. 2009;106:13094-9.

23. Parkkila S, Rajaniemi H, Parkkila AK, Kivela J, Waheed A, Pastorekova S, et al. Carbonic anhydrase inhibitor suppresses invasion of renal cancer cells in vitro. Proc Natl Acad Sci U S A. 2000;97:2220-4.

24. Lambin P, Petit SF, Aerts HJ, van Elmpt WJ, Oberije CJ, Starmans MH, et al. The ESTRO Breur Lecture 2009. From population to voxel-based radiotherapy: exploiting intra-tumour and intra-organ heterogeneity for advanced treatment of non-small cell lung cancer. Radiother Oncol. 2010;96:145-52.

25. Pacchiano F, Aggarwal M, Avvaru BS, Robbins AH, Scozzafava A, McKenna R, et al. Selective hydrophobic pocket binding observed within the carbonic anhydrase II active site accommodate different 4-substituted-ureido-benzenesulfonamides and correlate to inhibitor potency. Chem Commun (Camb). 2010;46:8371-3. 



\section{Chapter 7}

\section{Targeting carbonic anhydrase IX by}

nitroimidazole based sulfamides enhances the therapeutic effect of tumor irradiation: a new concept of dual targeting drugs

Published in: Radiother Oncol. 2013;108:523-8

Ludwig Dubois, Sarah G.J.A. Peeters*, Simon J.A. van Kuijk*, Ala Yaromina, Natasja G. Lieuwes, Ruchi Saraya, Rianne Biemans, Marouan Rami, Nanda Kumar Parvathaneni, Daniela Vullo, Marc Vooijs, Claudiu T. Supuran, Jean-Yves Winum and Philippe Lambin *contributed equally 


\section{Abstract}

Background and Purpose: Carbonic anhydrase IX (CAIX) plays an important role in $\mathrm{pH}$ regulation processes critical for tumor cell growth and metastasis. We hypothesize that a dual targeting bioreductive nitroimidazole based anti-CAIX sulfamide drug (DH348) will reduce tumor growth and sensitize tumors to irradiation in a CAIX dependent manner.

Material and M ethods: The effect of the dual targeting anti-CAIX (DH348) and its single targeting control drugs on extracellular acidification and radiosensitivity was examined in HT-29 colorectal carcinoma cells. Tumor growth and time to reach $4 x$ start volume ( $\mathrm{T} 4 \mathrm{xSV}$ ) was monitored for animals receiving DH348 $(10 \mathrm{mg} / \mathrm{kg})$ combined with tumor single dose irradiation (10 Gy).

Results: In vitro, DH348 reduced hypoxia-induced extracellular acidosis, but did not change hypoxic radiosensitivity. In vivo, DH348 monotherapy decreased tumor growth rate and sensitized tumors to radiation (enhancement ratio 1.50) without systemic toxicity only for CAIX expressing tumors.

Conclusions: A newly designed nitroimidazole and sulfamide dual targeting drug reduces hypoxic extracellular acidification, slows down tumor growth at nontoxic doses and sensitizes tumors to irradiation all in a CAIX dependent manner, suggesting no "off-target" effects. Our data therefore indicate the potential utility of a dual drug approach as a new strategy for tumor-specific targeting. 


\section{Introduction}

Elevated carbonic anhydrase (CA) IX expression has been associated with poor prognosis, tumor progression and aggressiveness (1). The plasma membrane associated dimeric glycoprotein catalyzes the reversible hydration of carbon dioxide to bicarbonate and a proton. CAIX is therefore an important component of the $\mathrm{pH}$ regulation systems, which are activated upon anaerobic glycolysis (2). Its main function during hypoxia is to preserve a neutral intracellular $\mathrm{pH}$ favorable for tumor growth and survival, while contributing to an acidic extracellular tumor microenvironment favorable for invasiveness $(3,4)$. Since CAIX is implicated in both extra - and intracellular $\mathrm{pH}$ regulation, it has been proposed as a potential therapeutic target.

A possible approach to target CAIX would be via inhibiting its enzymatic activity with specific pharmacological inhibitors $(2,5)$. Previously, we have demonstrated that inhibitors of CAIX activity require both protein expression and activation and this is dependent on the tumor oxygenation status (6-8). Tumor-specific accumulation was found after administration of fluorescent sulfonamides at low dosage, while inhibition of tumor growth was observed at higher dose levels (7, 9-11). Treatment of mammary tumor-bearing mice with novel CAIX-specific (ureido)-sulfonamide and glycolsylcoumarins inhibitors resulted in a significant inhibition of primary tumor growth and lung metastasis formation (11). Recently, an underexplored class of sulfamate inhibitors proved to be excellent candidates for low dosage anti-metastatic drugs in breast cancer therapy (12). These reports emphasize that specific inhibitors of CAIX activity are promising to pursue for their tumor-specific therapeutic properties in combination with conventional therapies. Recently, the CAIX dependent sensitizing effect of indanesulfonamides (8) and acetazolamide (13) on respectively radio- and chemotherapy has been demonstrated.

A different approach to sensitize tumors to irradiation is the use of the 2nitroimidazole based hypoxic radiosensitizer misonidazole. This has shown to improve the radiation response both when administered in a single or fractionated radiation scheme, however with high clinical toxicity (14). The less toxic 5-nitroimidazole derivative, nimorazole, has shown to reach similar enhancement ratios as misonidazole in fractionated schedules (14) and resulted in a highly significant benefit in terms of loco-regional tumor control and disease-free survival (15). More specific targeting towards the hypoxic tumor regions using lower doses is therefore an important requisite for new compounds. 
The aim of this study was to evaluate the antitumor activity of a newly designed class of dual targeting nitroimidazole based sulfamide drugs with high affinity for CAIX. We hypothesize that these compounds specifically block CAIX activity, reduce tumor growth and sensitize tumors to irradiation in a CAIX dependent manner.

\section{Material and Methods}

\section{Cell culture and model}

Exponentially growing colorectal (HT-29, ATCC HTB-38) carcinoma cells were cultured in Dulbecco's modified Eagle's medium supplemented with $10 \%$ fetal bovine serum. HT-29 colorectal carcinoma cells harboring a shCAIX (KD) or control shRNA (EV) construct (kindly provided by Adrian Harris, Weatherall Institute of Molecular Medicine, University of Oxford, John Radcliffe hospital, Oxford, UK) were engineered as previously described (8). Low oxygen conditions were acquired in a hypoxic workstation (Ruskinn INVIVO 2 1000). The atmosphere in the chamber consisted of $0.2 \% \mathrm{O}_{2}$ (hypoxia) or $<0.02 \% \mathrm{O}_{2}$ (anoxia, $0 \%$ ), $5 \% \mathrm{CO}_{2}$ and residual $\mathrm{N}_{2}$. In parallel, normoxic $(20 \%$ $\mathrm{O}_{2}$ ) dishes were incubated in air with $5 \% \mathrm{CO}_{2}$. $\mathrm{pH}$ of the culture medium was immediately measured at the end of each experiment as previously described (6).

\section{Inhibition CAIX activity}

The structure and $\mathrm{Ki}$ value of the different compounds are depicted in figure 7.1A. Compounds were dissolved in culture medium containing $0.5 \%$ DMSO at the indicated final concentrations just before addition to the cells. For the animal experiments, DH348 was dissolved in $\mathrm{NaCl} 0.9 \%$ containing $1 \%$ DMSO to a final concentration of 10 $\mathrm{mg} / \mathrm{kg}$ and injected intravenously via a lateral tail vein.

\section{Immunoblotting}

Experiments were performed as previously described (6). Antibodies used were M75 (kindly provided by Silvia Pastorekova, Institute of Virology, Slovak Academy of Science, Bratislava, Slovak Republic) against CAIX or $\beta$-actin (Cell Signaling) as loading control. 


\section{Clonogenic survival}

Cells were exposed to normoxia or anoxia for $24 \mathrm{~h}$, subsequently irradiated (225 kV Philips X-ray tube), trypsinized and plated in triplicate for clonogenic survival. Cells were allowed to form colonies during 10 days, which were fixed and stained with $0.4 \%$ methylene blue in $70 \%$ ethanol. Afterwards, colonies were counted in an automated way (Oxford Optronix).

\section{Animal experiments}

All experiments were in accordance with local institutional guidelines for animal welfare and were approved by the Animal Ethical Committee of the University. 1.5e6 cells were resuspended in Basement Membrane Matrix (Matrigel ${ }^{\mathrm{TM}}$ BD Biosciences) and injected subcutaneously into the lateral flank of adult NMRI-nu mice (28-32 g). Intravenous DH348 treatment started at a tumor volume of $193 \pm 66 \mathrm{~mm}^{3}$ for 5 days (10 mg/kg daily) and irradiation (10 Gy single dose (8)) was performed at day 3 . Tumor growth was monitored until reaching $4 x$ the volume at irradiation time (T4xSV) and treatment toxicity was scored by body weight measurements. Radiation sensitization enhancement ratios (SER) are calculated as the ratios of the mean T4xSV of the DH348 compared to the vehicle treated irradiation groups.

\section{Immunohistochemistry}

Frozen sections were fixed in $4 \%$ formalin and incubated with normal goat serum. Contiguous sections were stained using rabbit anti-pimonidazole (1:250, NPI Inc) or rabbit anti-CAIX (1:1000, Novus Biologicals). Secondary incubation was done with biotinylated goat anti-rabbit antibody (1:200 and 1:400 for CAIX and pimonidazole respectively, DAKO). Detection was done with Vectastain ABC kit (Vector Laboratories) followed by $0.033 \%$ hydrogen peroxide in $10 \%$ diaminobenzidine (DAB; Sigma-Aldrich). Staining without primary antibody served as negative control. The sections were viewed (magnification 4x) by means of a Nikon Eclipse E800 microscope (Nikon Instruments Inc).

\section{Statistics}

All statistical analyses were performed with GraphPad Prism version 5.03 for Windows (GraphPad Software, 2009, California, USA). A non-parametric Mann-Whitney U test for small groups was used to determine the statistical significance of differences 
between two independent groups of variables. For all tests, a $\mathrm{P}<0.05$ was considered significant.

\section{Results}

HT-29 colorectal carcinoma cells showed elevated CAIX protein levels in response to anoxia (figure 7.1B) and its expression is heterogeneous across tumors, overlapping the pimonidazole-positive hypoxic areas (figure 7.1C). To examine the necessity for CAIX activity for extracellular acidification under hypoxic conditions, the effect of the dual targeting compound $\mathrm{DH} 348$ on hypoxia-induced changes in extracellular pH was tested. A significant dose-dependent $(P<0.05)$ reduction in hypoxia-induced extracellular acidosis was observed, while the effect on cells exposed to ambient air was negligible (figure 7.1D). To explore the effect of CAIX activity inhibition on tumor growth and radiotherapy response, HT-29 tumor bearing mice were treated with DH348 and subsequently irradiated. Tumor growth was significantly $(P<0.01)$ inhibited in DH348-treated mice versus mice receiving vehicle only. The time to reach $4 x$ irradiation starting volume $(T 4 x S V)$ was increased $(P<0.001)$ when treated with single dose irradiation, which was further enhanced $(P<0.05)$ upon combination with $\mathrm{DH} 348$ (figure $7.1 \mathrm{E}$ ) resulting in a radiation sensitization enhancement ratio (SER) of 1.50 .

To establish a causal relationship between CAIX expression and its therapeutic effect in more detail, CA9 was genetically silenced in a constitutive manner. A clear reduction in CAIX protein levels was observed for the CAIX knock-down (KD) cell line as compared with a control shRNA (EV) cell line (figure 7.2A). HT-29 EV xenografts demonstrated a robust and heterogeneous CAIX expression, which co-localized with the hypoxia marker pimonidazole. CAIX levels were dramatically reduced in KD xenografts, however without affecting the presence of hypoxia as assessed with pimonidazole (figure 7.2B). Next we examined whether CAIX depletion in vitro resulted in a decreased extracellular acidification upon hypoxia. Extracellular acidosis upon hypoxia was significantly reduced (30\%, $\mathrm{P}<0.01$ ) upon CAIX depletion (figure $7.2 \mathrm{C}$ ). Furthermore, we investigated if pharmacological inhibition of CAIX activity would mimic the effect of CAIX silencing. Besides the dual targeting drug DH348, the single targeting controls (NKP46 = 5-nitroimidazole: 5 -NI and NKP64 = sulfamide: Sulf) were evaluated for their capacity to reduce the CAIX dependent extracellular acidification. Both DH348 and Sulf reduced the hypoxic extracellular acidosis (43\% and 29\% for 1.0 and $0.1 \mathrm{mM}$ respectively, $\mathrm{P}<0.05$ ) to similar levels as seen for the KD cells. No effect 
A<smiles>Cc1ncc([N+](=O)[O-])n1CCNS(=O)(=O)[O-]</smiles><smiles>Cc1nnc([N+](=O)[O-])n1CCNS(C)(=O)=O</smiles><smiles>Cc1nccn1CCCNONS(=O)(=O)[O-]</smiles>

\begin{tabular}{|c|c|c|c|}
\hline \multirow[b]{2}{*}{ compound } & \multicolumn{3}{|c|}{$\kappa(\mathrm{nM})$} \\
\hline & nCAI & heall & hCA $1 x$ \\
\hline DH348 & DETS & 10.1 & 20.4 \\
\hline SKPAS IS-N & 750000 & .20000 & $\$ 20000$ \\
\hline
\end{tabular}

DH348 NKP 46 (SNN)

NKP54 (But)

B

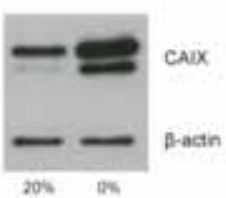

C

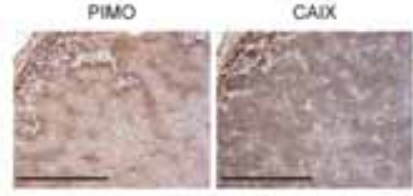

contrel

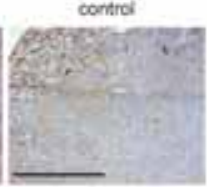

D

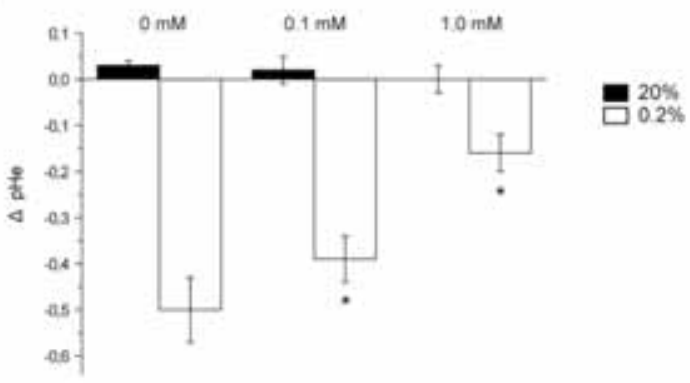

$\mathrm{E}$

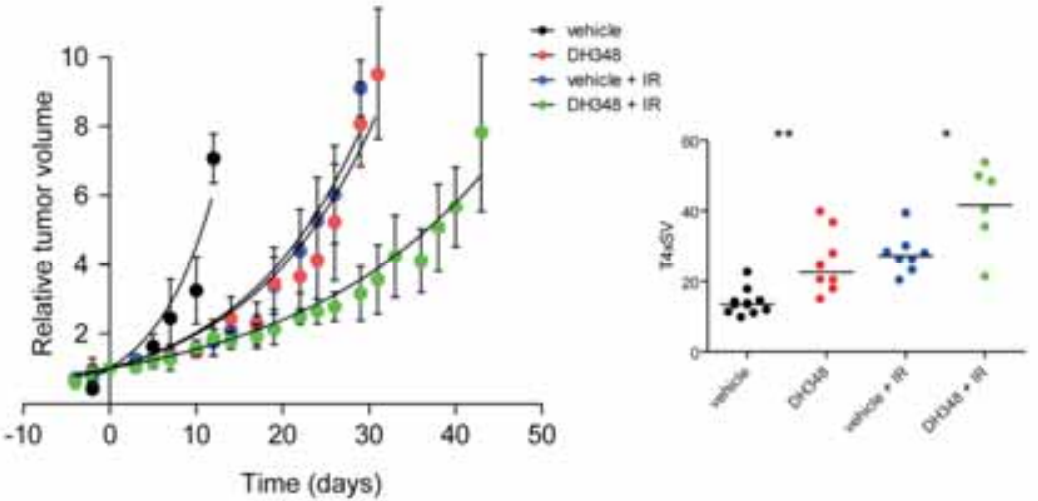

Figure 7.1 (A) Chemical structure and affinity (Ki) for CAI, CAll and CAIX of the dual targeting compound DH348 and its respective single targeting controls. (B) Western blot analysis of CAIX expression in HT-29 cells under normoxic (20\%) and anoxic (0\%) conditions. $\beta$-Actin was used as loading control. (C) Representative pimonidazole (PIMO) and CAIX immunohistochemical staining of HT-29 tumors resected 5 days after inhibitor treatment. Negative control indicates staining without the primary antibody. Bar indicates $1 \mathrm{~mm}$ length. (D) Extracellular acidification of cells exposed to normoxia (20\%) and hypoxia (0.2\%) upon treatment with 0.1 or $1.0 \mathrm{mM} \mathrm{DH348.} \mathrm{Data} \mathrm{show} \mathrm{the} \mathrm{mean} \pm$ SD of at least three independent experiments and are expressed as the difference between pHe values $\left(\Delta \mathrm{pHe}=\mathrm{pH}_{\text {after incubation }}-\mathrm{pH}_{\text {before incubation }}\right)$. (E) DH348 (10 $\mathrm{mg} / \mathrm{kg}, 5$ days) was administered intravenously when tumors reached an average size of $200 \mathrm{~mm}^{3}$ and tumors were irradiated (10 Gy) at day 3 (set to 0 ) of this treatment. Tumor growth was monitored until reaching $4 x$ the volume at irradiation time (T4xSV). Data represent the mean \pm SD of six to nine independent animals. Asterisks indicate statistical significance ( $\left.{ }^{*} \mathrm{P}<0.05 ; * * \mathrm{P}<0.01\right)$. 
A

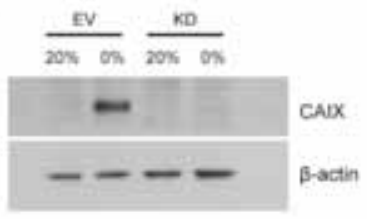

B

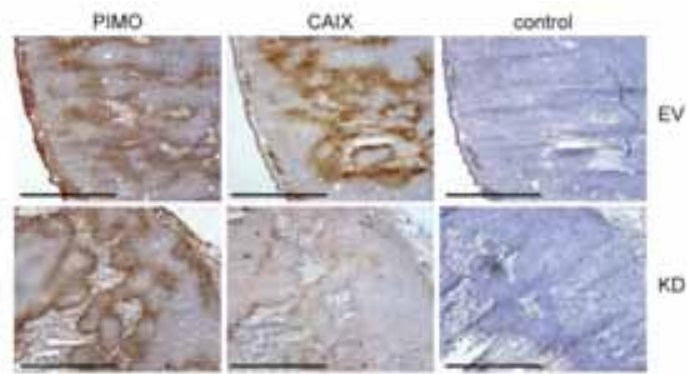

C
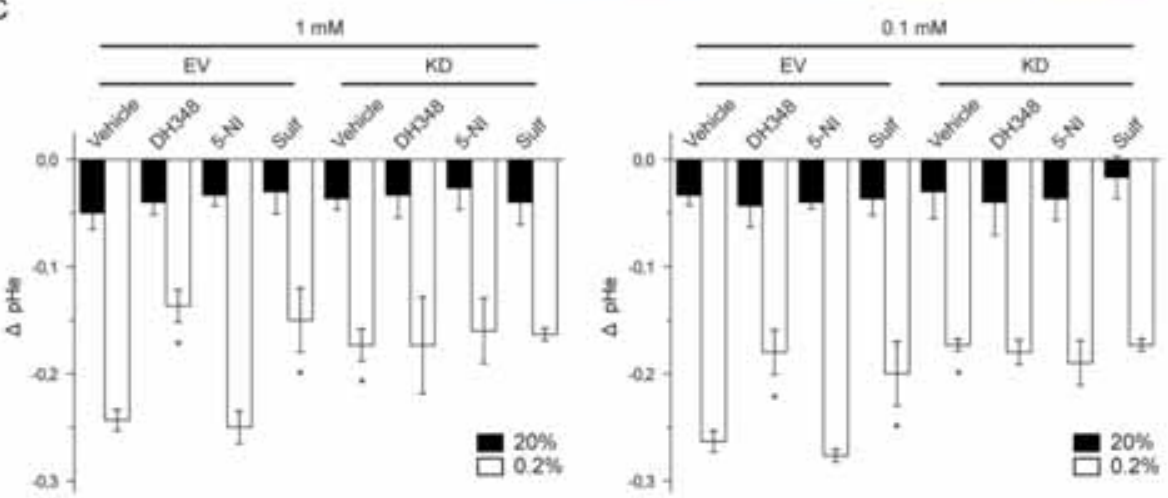

D
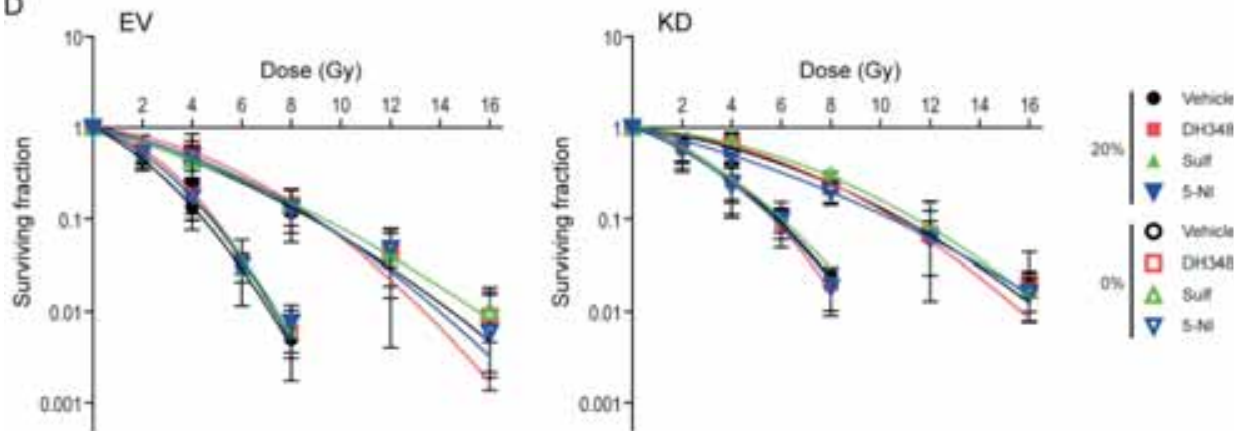

Figure7.2 (A) Western blot analysis of CAIX expression in HT-29 CAIX expressing (EV) and CAIX silenced (KD) cells under normoxic (20\%) and anoxic (0\%) conditions. (B) Representative pimonidazole (PIMO) and CAIX immunohistochemical staining of EV and KD tumors resected 5 days after inhibitor treatment. (C) Extracellular acidification of EV and KD cells exposed to normoxia (20\%) and hypoxia (0.2\%) upon treatment with 0.1 or $1.0 \mathrm{mM}$ DH348 or controls. Data represent the mean \pm SD of at least three independent experiments. (D) Clonogenic survival assay after exposure to different doses of irradiation upon normoxia (20\%: 0, 2, 4, 6 and $8 \mathrm{~Gy}$ ) and anoxia (0\%: 0, 4, 8, 12 and $16 \mathrm{~Gy}$ ) exposed $\mathrm{EV}$ and $\mathrm{KD}$ cells after pre-treatment (24h) with 33 $\mu \mathrm{M}$ DH348 or controls. Data show mean \pm SD of at least three independent experiments. Asterisks indicate statistical significance compared to EV treated with vehicle under hypoxia $\left({ }^{*} \mathrm{P}<0.05 ;{ }^{*} \mathrm{P}<0.01\right)$. 

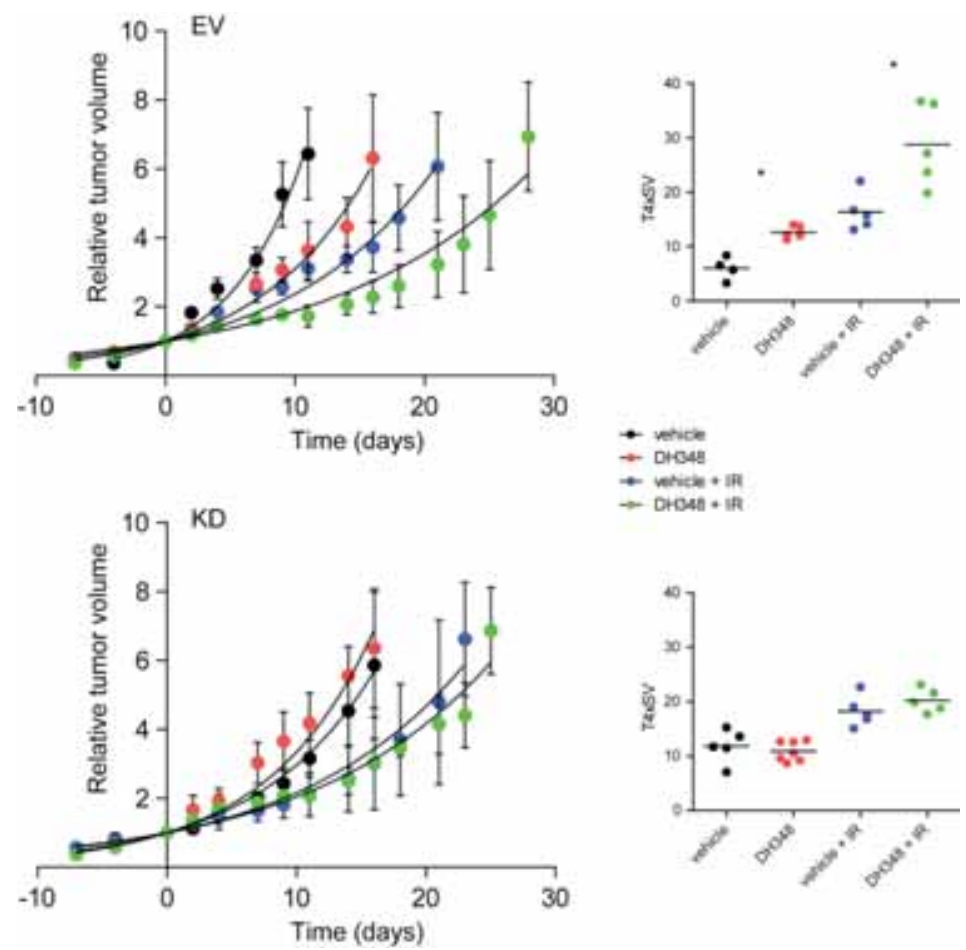

$\rightarrow$ Drost + H

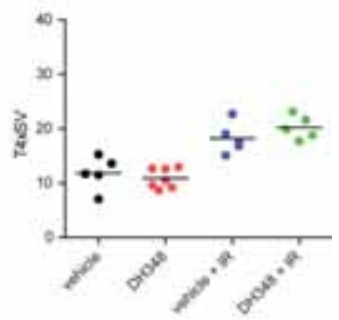

Figure7.3 DH348 (10 mg/kg, 5 days) was administered to EV or KD tumor-bearing animals when tumors reached an average size of $200 \mathrm{~mm}^{3}$ and tumors were irradiated (10 Gy) at day 3 (set to 0 ) of this treatment. Tumor growth was monitored until reaching $4 x$ the volume at irradiation time (T4xSV). Data represent the mean \pm SD of five to eight independent animals. Asterisks indicate statistical significance $\left(* \mathrm{P}<0.05 ;{ }^{* *} \mathrm{P}<\right.$ 0.01).

was seen when cells were incubated in ambient air or upon addition to KD cells. Similarly, 5-NI did not affect extracellular acidosis under any experimental conditions (figure 7.2C). To examine if inhibition of CAIX activity influences radiosensitivity, the in vitro response of EV and KD cells to varying radiation doses after pre-incubation with the different compounds was measured under normoxia and anoxia (figure 7.2D). As expected, both cell lines demonstrated a more radioresistant phenotype under anoxic conditions. However, none of the compounds when added before irradiation resulted in enhancement of the irradiation effect, suggesting the need for a naive tumor microenvironment.

To exclude non-specific activity of the dual targeting compound in vivo when investigating the effect of CAIX inhibition on radiation response, growth delay experiments were repeated for EV and KD xenografts. All animals were treated with DH348 at a similar volume for both tumor models. DH348 treatment resulted in a 
significant growth delay $(P<0.01)$ compared to vehicle controls and sensitized EV tumors to radiation $(P<0.05)$ with a SER of 1.45. These effects were only observed for the CAIX expressing EV tumor model, supporting the specificity of the CAIX targeted treatment (figure 7.3). Additionally, no body weight loss was observed for any of the treatment schedules suggesting no observable systemic toxicity (figure. S7.1).

\section{Discussion}

CAIX has been associated with cancer progression, metastasis and poor prognosis through its capacity to regulate intra - and extracellular acidity of hypoxic tumors $(1,4)$ and is therefore a potential therapeutic target. Recently, CAIX inhibitors have been proposed as potential anti-tumor agents $(2,5)$. Previously, others and we have demonstrated that not only CAIX expression, but also hypoxia is a requirement to enable accumulation of different classes of CAIX inhibitors (3, 6-8). Hypoxia causes an acidification of the extracellular environment and studies have demonstrated that CAIX is the main contributor to this process $(3,8,16)$. Also in the current study, genetic silencing of CAIX reduced the hypoxia-induced extracellular acidosis. Additionally, blocking CAIX activity using sulfamide-based CAIX inhibitors was also able to reduce extracellular acidification up to $40 \%$ only upon hypoxia in a CAIX-dependent manner, in agreement with previously obtained results using sulfonamide-based inhibitors $(6,8)$.

Hypoxia is a common feature of solid tumors and experimental and clinical studies have demonstrated that its presence is an important factor influencing tumor resistance to radiation therapy $(17,18)$. An extensively investigated approach to sensitize tumor to irradiation, is the use of nitroimidazole-based hypoxic radiosensitizers. The 2-nitroimidazole misonidazole has been shown to be a very efficient radiosensitizer in experimental and human tumors, however associated with unacceptable systemic toxicity, which prevented the drug to be given in therapeutically relevant doses (14). The less toxic 5-nitroimidazole derivative, nimorazole, has been shown to reach similar sensitizer enhancement ratios (SER 1.26) in a fractionated radiation schedule ( $300 \mathrm{mg} / \mathrm{kg}$ ) as compared to misonidazole (SER 1.32) but with far less toxicity (14) and is therefore now used in standard clinical treatment for head and neck cancer patients in Denmark (15). Recently, it has been shown that the tumor response to fractionated irradiation is also dependent on the glycolytic metabolism and therefore on extracellular acidosis (19). We have previously shown that CAIX inhibition using sulfonamide $11 \mathrm{c}$ is able to counteract the hypoxic-induced extracellular acidification resulting in a sensitization to radiation (8). Times to reach 4 
times the starting volumes (T4xSV) were 32.5 (11c) vs 27.8 (vehicle) days for HT-29 parental and 22.2 (11c) vs 17.2 (vehicle) days for EV xenografts (8), resulting in a respective SER of 1.17 and 1.29.

We therefore designed a dual targeting CAIX-specific compound (DH348) composed of a 5-nitroimidazole radiosensitizing hypoxic cells and a sulfamide targeting active CAIX. We hypothesize that this compound shows an enhanced sensitization to irradiation in a CAIX-dependent manner. Reduced CAIX expression was observed in KD xenografts without affecting the presence of hypoxia as assessed with pimonidazole staining. $A$ recent study reported an enhanced pimonidazole labeling upon CAIX silencing. However, this association was only found in spheroids, but not in xenograft models, emphasizing the requirement of host tissue (20). Single treatment with DH348 resulted in a slower tumor growth compared to vehicle treatment only in CAIX expressing tumors. Similar results were obtained in mammary or colorectal tumor-bearing mice for which the effect is dependent on the treatment schedule and the administered dose $(8,9,11)$. In contrary, a new class of CAIX-specific sulfamate inhibitors did not affect primary tumor growth at low doses, but had significant anti-metastatic properties in breast cancer therapy (12). Recently, the general CA inhibitor acetazolamide has been found to enhance the toxicity of the chemotherapeutic agent doxorubicin, with the highest effect for CAIX expressing cell lines (13). Therefore, it is promising to pursue specific inhibitors of CAIX activity for their capacity to enhance the effect of conventional therapies.

In the current study, upon treatment with the CAIX-specific dual targeting sulfamide DH348, the therapeutic effect of irradiation was enhanced in a CAIX dependent manner (SER 1.50 and 1.45 for HT-29 parental and EV tumors respectively) without systemic toxicity as evaluated by body weight loss. Higher SER was found for parental HT-29, which might be explained by higher CAIX activity, since hypoxic extracellular acidification was larger compared to the EV tumor cells. Furthermore, no sensitization (SER 1.04) could be observed for the KD tumors. The enhancement ratios found in the present study are $30 \%$ higher compared with the previously investigated indanesulfonamide (8). It can be argued that we did not include the single targeting agents in our animal study. Previously, we have demonstrated that a single CAIX targeting agent showed smaller sensitization effects compared with the currently investigated dual targeting drug (vide supra) (8). Additionally, we observed that the SER of the dual targeting compound DH348 (10 mg/kg) was also higher than the well-established hypoxic radiosensitizers misonidazole and nimorazole for which a minimal dose of 100 
$\mathrm{mg} / \mathrm{kg}$ was needed to achieve an SER above unity (14). In vitro, no hypoxic radiosensitization was observed using previously described effective dose regimens (12). Since also no radiosensitization was found for the 5-NI single compound when using equimolar doses, in vitro drug dose levels might be too low. Nimorazole has been shown to radiosensitize tumor cells, but this at dose levels 30 fold higher than used in the current study (21). We are currently investigating if higher drug doses can radiosensitize hypoxic cells in vitro. In the present proof-of-principle study only one tumor line was investigated and single dose was applied, which warrants confirmatory investigations using tumor models of other histology and fractionated radiation schemes. These experiments as well as mechanistic studies have been initiated in our laboratory. In order to prepare the first in human clinical trial, pharmacokinetic, pharmacodynamic and extensive toxicity studies are currently ongoing.

In conclusion, a newly designed nitroimidazole and sulfamide based dual targeting drug is able to reduce hypoxic extracellular acidification, to inhibit tumor growth at nontoxic doses and to sensitize tumors to irradiation in a CAIX dependent manner proving the absence of any "off-target" effect. The dual drug enhances radiation response more effectively as compared to single published CAIX targeting compounds. Our data therefore indicate the potential utility of a dual drug approach as a new strategy for tumor-specific targeting to eventually improve the response to radiation treatment.

\section{Acknowledgements}

This work has been funded with the support of the Maastro Cancer Foundation, KWF UM2012-5394 and the EU $7^{\text {th }}$ framework (Metoxia project ref. 2008-222741). 


\section{Corrigendum for:}

Targeting carbonic anhydrase IX by nitro-imidazole based sulfamides enhances the therapeutic effect of tumor irradiation: A new concept of dual targeting drugs. j.radonc.2013.06.018 (Dubois et al., 2013)

In this article (Volume 108, Issue 3, Pages 523-528) published in the September 2013 issue of Radiotherapy and Oncology, we reported the use HT-29 EV (empty vector) and shCA9 (Carbonic anhydrase IX targeting shRNA) cell lines. Using Short tandem repeat (STR) analysis we recently discovered that these cell lines are of HCT116 origin instead another colon cancer cell line. Since the proper empty vector control CAIX expressing cell line was included in the paired cell lines for both publications, our conclusion stating that the therapeutic efficacy of blocking CAIX activity is causally dependent on CAIX expression is still valid. The authors regret this error.

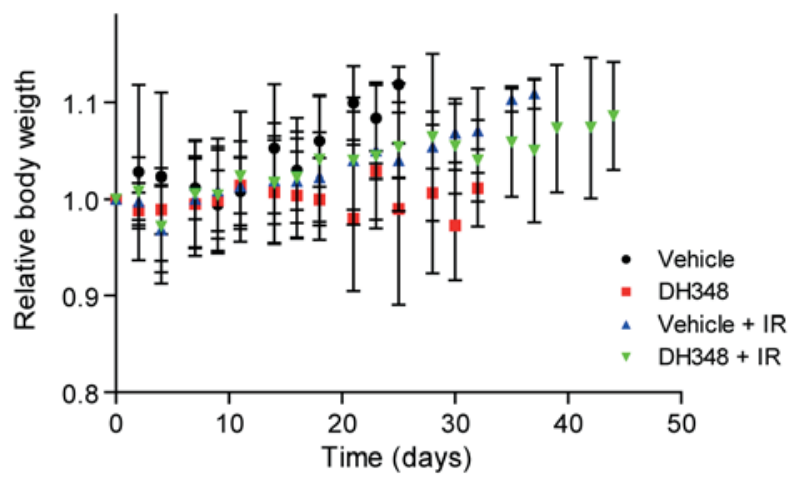

Supplementary figure 7.1 DH348 (10 mg/kg, 5 days) was administered to animals when tumors reached an average size of $200 \mathrm{~mm} 3$ and tumors were irradiated ( $10 \mathrm{~Gy}$ ) at day 3 (set to 0 ) of this treatment. Treatment toxicity was evaluated by monitoring body weight $3 x /$ week. Data represent the mean \pm SD of all animals within a treatment group (independent of the type of tumor). 


\section{References}

1. Potter CP, Harris AL. Diagnostic, prognostic and therapeutic implications of carbonic anhydrases in cancer. Br J Cancer. 2003;89:2-7.

2. McDonald PC, Winum JY, Supuran CT, Dedhar S. Recent developments in targeting carbonic anhydrase IX for cancer therapeutics. Oncotarget. 2012;3:84-97.

3. Svastova E, Hulikova A, Rafajova M, Zat'ovicova M, Gibadulinova A, Casini A, et al. Hypoxia activates the capacity of tumor-associated carbonic anhydrase IX to acidify extracellular pH. FEBS Lett. 2004;577:439-45.

4. Swietach P, Patiar S, Supuran CT, Harris AL, Vaughan-Jones RD. The role of carbonic anhydrase 9 in regulating extracellular and intracellular $\mathrm{pH}$ in three-dimensional tumor cell growths. J Biol Chem. 2009;284:20299-310.

5. Neri D, Supuran CT. Interfering with $\mathrm{pH}$ regulation in tumours as a therapeutic strategy. Nat Rev Drug Discov. 2011;10:767-77.

6. Dubois L, Douma K, Supuran CT, Chiu RK, van Zandvoort MA, Pastorekova S, et al. Imaging the hypoxia surrogate marker CA IX requires expression and catalytic activity for binding fluorescent sulfonamide inhibitors. Radiother Oncol. 2007;83:367-73.

7. Dubois L, Lieuwes NG, Maresca A, Thiry A, Supuran CT, Scozzafava A, et al. Imaging of CA IX with fluorescent labelled sulfonamides distinguishes hypoxic and (re)-oxygenated cells in a xenograft tumour model. Radiother Oncol. 2009;92:423-8.

8. Dubois L, Peeters S, Lieuwes NG, Geusens N, Thiry A, Wigfield S, et al. Specific inhibition of carbonic anhydrase IX activity enhances the in vivo therapeutic effect of tumor irradiation. Radiother Oncol. 2011;99:424-31.

9. Ahlskog JK, Dumelin CE, Trussel S, Marlind J, Neri D. In vivo targeting of tumor-associated carbonic anhydrases using acetazolamide derivatives. Bioorg Med Chem Lett. 2009;19:4851-6.

10. Buller F, Steiner M, Frey K, Mircsof D, Scheuermann J, Kalisch M, et al. Selection of Carbonic Anhydrase IX Inhibitors from One Million DNA-Encoded Compounds. ACS chemical biology. 2011;6:336-44.

11. Lou Y, McDonald PC, Oloumi A, Chia S, Ostlund C, Ahmadi A, et al. Targeting tumor hypoxia: suppression of breast tumor growth and metastasis by novel carbonic anhydrase IX inhibitors. Cancer Res. 2011;71:3364-76.

12. Gieling RG, Babur M, Mamnani L, Burrows N, Telfer BA, Carta F, et al. Antimetastatic effect of sulfamate carbonic anhydrase IX inhibitors in breast carcinoma xenografts. J Med Chem. 2012;55:5591-600.

13. Gieling RG, Parker CA, De Costa LA, Robertson N, Harris AL, Stratford IJ, et al. Inhibition of carbonic anhydrase activity modifies the toxicity of doxorubicin and melphalan in tumour cells in vitro. Journal of enzyme inhibition and medicinal chemistry. 2013;28:360-9.

14. Overgaard J, Overgaard M, Nielsen OS, Pedersen AK, Timothy AR. A comparative investigation of nimorazole and misonidazole as hypoxic radiosensitizers in a $\mathrm{C} 3 \mathrm{H}$ mammary carcinoma in vivo. $\mathrm{Br} \mathrm{J}$ Cancer. 1982;46:904-11.

15. Overgaard J, Hansen HS, Overgaard M, Bastholt L, Berthelsen A, Specht L, et al. A randomized doubleblind phase III study of nimorazole as a hypoxic radiosensitizer of primary radiotherapy in supraglottic larynx and pharynx carcinoma. Results of the Danish Head and Neck Cancer Study (DAHANCA) Protocol 5-85. Radiother Oncol. 1998;46:135-46.

16. van den Beucken $\mathrm{T}$, Ramaekers $\mathrm{CH}$, Rouschop $\mathrm{K}$, Koritzinsky $\mathrm{M}$, Wouters BG. Deficient carbonic anhydrase 9 expression in UPR-impaired cells is associated with reduced survival in an acidic microenvironment. Radiother Oncol. 2009;92:437-42. 
17. Nordsmark M, Bentzen SM, Rudat V, Brizel D, Lartigau E, Stadler P, et al. Prognostic value of tumor oxygenation in 397 head and neck tumors after primary radiation therapy. An international multicenter study. Radiother Oncol. 2005;77:18-24.

18. Yaromina A, Eckardt A, Zips D, Eicheler W, Schuetze C, Thames H, et al. Core needle biopsies for determination of the microenvironment in individual tumours for longitudinal radiobiological studies. Radiotherapy and oncology : journal of the European Society for Therapeutic Radiology and Oncology. 2009;92:460-5.

19. Sattler UG, Meyer SS, Quennet V, Hoerner C, Knoerzer H, Fabian C, et al. Glycolytic metabolism and tumour response to fractionated irradiation. Radiother Oncol. 2010;94:102-9.

20. McIntyre A, Patiar S, Wigfield S, Li JL, Ledaki I, Turley H, et al. Carbonic anhydrase IX promotes tumor growth and necrosis in vivo and inhibition enhances anti-VEGF therapy. Clin Cancer Res. 2012;18:310011.

21. Sugie $C$, Shibamoto $Y$, Ito $M$, Ogino $H$, Suzuki $H$, Uto $Y$, et al. Reevaluation of the radiosensitizing effects of sanazole and nimorazole in vitro and in vivo. Journal of radiation research. 2005;46:453-9. 

Chapter 8

General discussion \& Future perspectives 
Hypoxia is a common characteristic of solid tumors and associated with worse treatment outcome. Therefore knowledge about the presence, amount and location of those hypoxic regions would be of great value. Furthermore, drugs specifically targeting those therapy-resistant hypoxic cells may complement conventional therapies like chemotherapy and radiotherapy. In this thesis both these tumor hypoxia related aspects were assessed with the ultimate goal to investigate if noninvasive monitoring of tumor hypoxia can predict the efficacy of anti-hypoxia treatment. This chapter discusses the different studies described in this thesis with respect to each other and the most recent literature. Lastly some future perspectives will be discussed.

\section{Monitoring tumor hypoxia}

Several hypoxia PET tracers have been developed over the last decades. Chapter 2 gives an overview of 2-nitroimidazole based hypoxia PET tracers that have been positively evaluated in both preclinical and clinical studies. Preclinical models form an ideal platform for initial validation and feasibility studies. Also, studies comparing tracers for their specific characteristics are more easily performed in preclinical settings.

$\left[{ }^{18} \mathrm{~F}\right] \mathrm{FMISO}$ was the first developed PET tracer and although it has some unfavorable characteristics, multiple preclinical comparative studies reveal $\left[{ }^{18} \mathrm{~F}\right] \mathrm{FMISO}$ as being superior or equal to newly developed tracers (1-3). The only tracers so far that outperform $\left[{ }^{18} \mathrm{~F}\right] \mathrm{FMISO}$ are $\left[{ }^{18} \mathrm{~F}\right] \mathrm{FAZA}$ and $\left[{ }^{18} \mathrm{~F}\right] \mathrm{HX} 4$. However, tracers had only been studied in separate preclinical models, making a direct comparison impossible. Chapter 3 discusses the first study comparing these three tracers in one preclinical tumor model with regard to the optimal imaging time point, tumor to blood ratios, reproducibility and sensitivity to oxygen modifications. It was observed that $\left[{ }^{18} \mathrm{~F}\right] \mathrm{FMISO}$ did not reach an optimal time point for imaging, but it had high reproducibility and sensitivity for a decreasing hypoxic fraction. $\left[{ }^{18}\right.$ F]FAZA did reach an optimal imaging time at $2 \mathrm{~h}$ p.i. and showed to be sensitive to an increased hypoxic fraction, however, in this study reproducibility for $\left[{ }^{18} \mathrm{~F}\right] \mathrm{FAZA}$ was worse. For $\left[{ }^{18} \mathrm{~F}\right] \mathrm{HX} 4$ the optimal imaging time point was at $3 \mathrm{~h}$ p.i. with the highest tumor to background ratio. Furthermore, $\left[{ }^{18} \mathrm{~F}\right] \mathrm{HX} 4$ demonstrated good reproducibility and sensitivity to an increase in hypoxic fraction. Only one other study so far investigated these three hypoxia PET tracers in a direct comparison; Carlin et al. assessed the relationship between tracer uptake of $\left[{ }^{18} \mathrm{~F}\right] \mathrm{FMISO},\left[{ }^{18} \mathrm{~F}\right] \mathrm{FAZA}$ and $\left[{ }^{18} \mathrm{~F}\right] \mathrm{HX} 4$ with immunohistochemical markers for hypoxia (pimonidazole), perfusion, and Carbonic Anhydrase (CAIX) (4). All three tracers showed 
clear uptake on PET. Moreover, good, comparable results were observed between tracer autoradiography and the pimonidazole uptake or CAIX expression. Although current available tracers have proven to be reliable in visualizing tumor hypoxia, it is not inconceivable that more tracers will be developed. For a proper testing of these tracers some guidelines should be followed. They should be compared with already existing PET tracers within one tumor model, furthermore, there applicability should be proven in multiple cancer types, a causal relationship with the oxygenation has to be demonstrated and validation with immunohistochemical hypoxia markers needs to be done.

Although preclinical models give a first indication, clinical validation of tracers is of importance because of pharmacokinetic variations between human and rodents. Furthermore in preclinical setting xenograft models are often used that do not fully represent the tumor at its original location. The feasibility of hypoxia detection of $\left[{ }^{18} \mathrm{~F}\right] \mathrm{FMISO},\left[{ }^{18} \mathrm{~F}\right] \mathrm{FAZA}$ and $\left[{ }^{18} \mathrm{~F}\right] \mathrm{HX} 4$ has also been demonstrated in clinical studies. Already in $1996\left[{ }^{18} \mathrm{~F}\right] \mathrm{FMISO}$ was used to visualize hypoxic tumors and quantify the hypoxic fraction in NSCLC patients (5). The feasibility of $\left[{ }^{18} \mathrm{~F}\right] \mathrm{FMISO}$ to detect hypoxia has been demonstrated in multiple cancer sites including brain (6), pancreatic (7), rectal (8), and renal (9) cancer. For $\left[{ }^{18} \mathrm{~F}\right]$ FAZA several studies have been conducted in $\operatorname{NSCLC}(10,11)$ and rectal cancer $(12)$. One study on prostate cancer has been published for $\left[{ }^{18} \mathrm{~F}\right] \mathrm{FAZA}$, but results were negative (13). Only few studies have investigated $\left[{ }^{18} \mathrm{~F}\right] \mathrm{HX} 4$ in clinical settings. First safety studies were performed in primates and healthy human volunteers (14), followed by a safety and feasibility study in patients with histologically proven solid cancer (15). A comparative study in head and neck cancer patients demonstrated that $\left[{ }^{18} \mathrm{~F}\right] \mathrm{HX} 4$ imaging at $1.5 \mathrm{~h}$ p.i. has similar tumor to muscle ratios as $\left[{ }^{18} \mathrm{~F}\right] \mathrm{FMISO}$ imaging at $2 \mathrm{~h}$ p.i (16). Moreover, two recently published papers by Zegers et al. assessed the most optimal imaging parameters of $\left[{ }^{18} \mathrm{~F}\right] \mathrm{HX} 4$ in non-small cell lung cancer (NSCLC). These studies demonstrated that imaging contrast is superior at $4 \mathrm{~h}$ p.i. compared to $2 \mathrm{~h}$ p.i. although uptake patterns correlated strongly between the two scans. A reduction of the scan time from 30 minutes to 10 minutes did not affect the detection of the hypoxic fraction, only when acquisition time was reduced to 5 minutes this induced a significant difference (17). Furthermore, overlap fractions between $\left[{ }^{18} \mathrm{~F}\right] \mathrm{HX} 4$ and the sugar analog $\left[{ }^{18} \mathrm{~F}\right] \mathrm{FDG}$ were calculated. The $\left[{ }^{18} \mathrm{~F}\right] \mathrm{FDG}$ positive volume was up to 3 times larger than the $\left[{ }^{18} \mathrm{~F}\right] \mathrm{HX} 4$ volume, and in $53 \%$ of the cases less than $1 \mathrm{~cm}^{3}$ of the hypoxia area was outside the $\left[{ }^{18} \mathrm{~F}\right]$ FDG region (18). Although there is an overlap between the areas detected with 
$\left[{ }^{18} \mathrm{~F}\right] \mathrm{FDG}$ and with $\left[{ }^{18} \mathrm{~F}\right] \mathrm{HX} 4$, this also indicates that both tracers clearly monitor different characteristics of the tumor and thereby complement each other.

In conclusion, these data suggest that all three tracer, $\left[{ }^{18} \mathrm{~F}\right] \mathrm{FMISO},\left[{ }^{18} \mathrm{~F}\right] \mathrm{FAZA}$ and $\left[{ }^{18} \mathrm{~F}\right] \mathrm{HX} 4$, are suitable for the noninvasive detection or tumor hypoxia. Furthermore, the clinically used $\left[{ }^{18} \mathrm{~F}\right] \mathrm{FDG}$ is proven to be a good tracer to detect tumors, however it does not accurately correspond with the hypoxic fraction in the tumor. Therefore $\left[{ }^{18}\right.$ F]FDG and hypoxia markers should be used complementary to each other.

Another approach to monitor tumor hypoxia is via surrogate hypoxia tracers that detect proteins that are upregulated in response to hypoxia. Directing a tracer to these proteins does not represent tumor hypoxia directly, but visualizes cells that have undergone a change upon low oxygen concentration. CAIX is a protein upregulated in response to hypoxia (19) and its expression is associated with worse clinical outcome (20). It has been demonstrated that the expression of CAIX is mainly located within the hypoxic regions; immunohistochemical staining for pimonidazole and CAIX give good overlap and also cells that are positive for hypoxia PET tracer uptake on autoradiography show a partial overlay with CAIX expression (4). However, there are also several studies that demonstrate the mismatch between pimonidazole assessed hypoxic regions and CAIX in both preclinical (21) and clinical studies (22). The cells expressing CAIX and located outside the hypoxic fraction could be reoxygenated, but due to the protein half-life of 38 hours the CAIX expression can still be there. Moreover, there is a difference in the capturing threshold between techniques capable of detecting hypoxia; pimonidazole has a threshold of $<10 \mathrm{mmHg}(4)$, while HIF stabilization occurs at oxygen levels $<20 \mathrm{mmHg}(23)$. Furthermore, since CAIX is also an attractive protein for targeting, direct monitoring of the presence of this protein will allow patient selection for additional treatment with specific CAIX inhibitors. The ability to monitor CAIX inhibitors by fluorescent labeling has been shown in preclinical setting (24), however this is not widely applicable in clinical setting.

In Chapter 4 a new molecule for monitoring CAIX activity, [ $\left.{ }^{18} \mathrm{~F}\right] \mathrm{VM} 4-037$, was investigated in two preclinical tumor models. Although in vitro results were favorable, no uptake from the CAIX expressing tumor was found in vivo. Metabolic analysis demonstrated no rapid degradation of the molecule, since 45 minutes post injection still $43 \%$ of the tracer was present. A possible explanation for the negative in vivo results could be attributed to the presence of the tumor microenvironment such as vasculature, surrounding tissue or nonspecific binding. Small inhibitors would have the 
ability to distinguish active CAIX from total CAIX expression, this in contrast to peptides, antibody fragments and full antibodies which detect the presence of CAIX. Full antibodies for the detection of CAIX expression do exist (25). Recently two newly developed tracers directed against CAIX have been evaluated positively. Both tracers, $\left[{ }^{18} \mathrm{~F}\right] 4 \mathrm{~d}$ based on peptides (26) and 99-Technetium labeled $(\mathrm{HE})_{3}$-ZCAIX:1 based on affibodies (27), are small. In contrast to full antibodies, small molecules distribute fast throughout the body and tumor and allow detection on relatively short notice. Further investigation of these tracers on, for instance, specificity for CAIX is needed before it can be introduced into the clinic.

Concluding, two ways of tumor hypoxia monitoring were investigated in this thesis. On the one hand the further characterization of the already existing hypoxia PET tracers while on the other hand a new surrogate hypoxia tracer was studied. Both approaches could be very useful in the monitoring of cancer patients, and providing useful information, however at this point the hypoxia PET tracers are more established and applicable in the clinical practice.

\section{Targeting hypoxia to improve radiotherapy}

Conventional anticancer therapies, radiotherapy and chemotherapy, are known to be less effective when cells are hypoxic. Therefore there is a need for additional drugs that specifically target these therapy-resistant cells. In this thesis two ways of specific hypoxia targeting were investigated; direct targeting of the hypoxia cells by a specific hypoxia-activated prodrug and targeting of the hypoxia response protein CAIX. It is unlikely that those drugs will induce cure as monotherapy, however, they could perfectly serve to complement the existing conventional anti-cancer therapies.

The concept of hypoxia-activated prodrugs (HAP) is based on the specific targeting of hypoxic regions without causing toxicity to well-oxygenated tissues. Several of these drugs have been designed and tested in preclinical and clinical setting. $\mathrm{TH}-302$, one of the new generation HAP, in combination with standard chemotherapy treatment has shown promising results in clinical trials (28-30). This in contradiction to a previously studied HAP, tirapazamine, which showed no additional treatment effect in a phase III clinical trial (31). In Chapter 5 the efficacy of TH-302 treatment in combination with radiotherapy was assessed for the first time. TH-302 as monotherapy clearly induces a tumor growth delay by specifically targeting of the hypoxic fraction. In combination with radiotherapy the effect on tumor growth is more pronounced than any of the 
therapies alone and can be modulated by altering the tumor oxygenation status. Other studies already showed a beneficial effect of TH-302 in combination with chemotherapeutics (29). The addition of TH-302 to the standard doxorubicin therapy in advanced soft tissue sarcoma (28) or gemcitabine treatment in pancreatic cancers (32) is already positively evaluated in a phase II clinical trial and phase III trials are ongoing. A clinical phase I trial for TH-302 to complement radiotherapy will start soon. These data indicate that $\mathrm{TH}-302$ is a new promising drug that specifically targets the hypoxic cells and thereby has the potential to complement conventional chemo- and radiotherapy treatments.

In chapter $\mathbf{6}$ and $\mathbf{7}$ targeting of the hypoxia response protein CAIX was studied. CAIX is upregulated in response to hypoxia and is known to play an important role in the regulation of the $\mathrm{pH}$. Maintenance of a stable intracellular $\mathrm{pH}$ is of great importance for the cell in order to survive. Moreover, the acidification of the extracellular environment contributes to a more aggressive behavior of the tumor cell $(33,34)$, and is also known to reduce the efficacy of conventional therapies (35). In Chapter 6 an indanesulfonamide $11 \mathrm{C}$ was shown to induce a tumor growth delay in combination with radiotherapy only in CAIX expressing tumors. In vitro an increased cleaved caspase 3 was detected indicating more apoptosis when CAIX was inhibited by the indanesulfonmide. Chapter 7 evaluated the dual-targeting drug DH348 consisting of both a sulfamide that is directed against CAIX and a 5-nitroimidazole that radiosensitizes hypoxic cells. By combining these two targeting mechanisms, this drug is thought to be extra specific for targeting the hypoxic cells. Before, 2-nitroimidazoles were found to be effective in sensitizing tumors to radiotherapy, however, with high clinical toxicity. It has been demonstrated that 5-nitroimidazoles were as effective, but with less toxic side effects (36). This dual targeting drug showed the ability to reduce the extracellular acidification in vitro. In vivo, a tumor growth delay was induced in CAIX expressing tumors which was further enhanced in combination with radiotherapy.

The difference in anti-tumor efficacy between the indanesulfonamide $11 \mathrm{C}$ and the dual targeting drug DH348 in combination with radiotherapy can be best compared by calculating the sensitization enhancement ratio (SER). In HT29 colorectal xenograft tumors the SER of $11 \mathrm{C}$ was 1.17 while a SER of 1.50 was reached using DH348. This demonstrates that the dual targeting drug is more effective. However, a disadvantage in this study is that the single moiety compounds were not taken along. This was only investigated in in vitro experiments and demonstrated that both the dual drug $\mathrm{DH} 348$ 
and the sulfonamide moiety had the capacity to reduce the acidification. Furthermore, the radiosensitizing capacity was assessed and although it was expected that the single 5-nitroimidazole compound would have an effect, this was not observed, due to the low dosing. Despite this limitation, data are promising and DH348 should be investigated further.

Both CAIX inhibitors showed an effect on tumor growth even without additional radiotherapy treatment. This is surprising since it wasn't expected that the inhibition of a single protein would induce such a detectable effect. Some literature suggests that inhibition of CAIX induces apoptosis (37). In chapter 6 an increased cleaved caspase 3 is detected upon the inhibition of CAIX under hypoxic conditions, indicating apoptosis. Furthermore there are several indications that CAIX is involved in other processes besides its function in $\mathrm{pH}$ regulation. The involvement of CAIX in the PI3/Akt pathway (38) and a potential function in the mTOR signaling (39) has been described. This pathway is known to be involved in cell survival, metabolism and tumor growth (40). In addition an interaction between CAIX and the anion exchanger (AE1) and the sodiumbicarbonate co-transporter (NBCe1) were found in a proximity ligation assay (41). These transmembrane transporters are responsible for the transport of the, via CAIX formed, bicarbonate back into the cell, thereby contributing to the maintenance of a stable intracellular $\mathrm{pH}$. All these studies indicate that CAIX is involved in more processes than initially was thought and endorse the potential of CAIX inhibition as anti-cancer therapy. Further research of the downstream processes, for instance by screenings on protein level, might be of importance and provide information that might be useful for the further development of CAIX inhibitors.

\section{Integrating hypoxia imaging in the treatment decision}

The ultimate goal of monitoring hypoxia is to use this information for prognostic and predictive purposes and when possible integrate this knowledge in complementing conventional treatment options to improve outcome. In daily practice monitoring of hypoxia is not yet applied, however, in preclinical and clinical studies this topic receives a lot of attention. As discussed in chapter $\mathbf{2}$ the prognostic value of hypoxia imaging has been assessed for different cancer types. Although there are some clinical studies that do not find an association (42), multiple studies have shown a positive correlation between hypoxia imaging and outcome. For head and neck cancer a combination of the nodal stage and $\left[{ }^{18}\right.$ F]FMISO PET imaging correlated significantly with survival (43), which was confirmed in another study correlating $\left[{ }^{18} \mathrm{~F}\right] \mathrm{FMISO}$ uptake and 
chemotherapy response (44). In addition, the prognostic value of hypoxia imaging was observed among others in glioma (45-47) and in breast cancer for the prediction of treatment resistance to hormonal therapy (48).

Although there are numerous positive publications on the prognostic and predictive value of hypoxia PET imaging, there are still some concerns about technical limitations and biological effects. One technical limitation is the resolution of PET scans. Hypoxia occurs at the cellular level, while the size of one PET voxel is approximately $5 \times 5 \times 5 \mathrm{~mm}^{3}$ and hence represents an average of all cells within this voxel. Immunohistochemical (IHC) markers, like pimonidazole and Hoechst, can be used to detect hypoxia and perfusion respectively on a microscopic level (49). Despite the differences in resolution between PET and IHC markers, a good correlation between pimonidazole uptake and tracer distribution has been observed for $\left[{ }^{18} \mathrm{~F}\right] \mathrm{FMISO}$ both on autoradiography or PET $(50,51),\left[{ }^{18} \mathrm{~F}\right] \mathrm{FAZA}(52,53)$ and $\left[{ }^{18} \mathrm{~F}\right] \mathrm{HX} 4(54)$, indicating that hypoxia PET imaging does give a good representation of the tumor's global oxygen status.

Another concern regarding hypoxia PET imaging is the reproducibility of a scan. A hypoxia PET image of a patient represents the oxygenation status of the tumor at that particular moment. When these images are used to generate personalized imageguided radiotherapy (IGRT) plans in which the hypoxic, radioresistant cells of the tumor will be boosted $(55,56)$, it would be desirable to have a tracer that shows stable uptake over time before the start of therapy to allow correct treatment planning. In chapter 3 the reproducibility of $\left[{ }^{18} \mathrm{~F}\right] \mathrm{FMISO},\left[{ }^{18} \mathrm{~F}\right] \mathrm{FAZA}$ and $\left[{ }^{18} \mathrm{~F}\right] \mathrm{HX} 4$ was detected within a 48 hour time frame, demonstrating good reproducibility for $\left[{ }^{18} \mathrm{~F}\right] \mathrm{FMISO}$ and $\left[{ }^{18} \mathrm{~F}\right] \mathrm{HX} 4$, while $\left[{ }^{18} \mathrm{~F}\right] \mathrm{FAZA}$ has poor reproducibility. However another study, using a cervix tumor model, shows good overlap between two $\left[{ }^{18} \mathrm{~F}\right] \mathrm{FAZA}$ pretreatment scans (53). Since these are preclinical studies, the relevance for the clinic has to be investigated. Furthermore, the reproducibility might be dependent on the tumor model, as suggested by the preclinical data.

Besides the reproducibility of a hypoxia PET image before treatment, the ability to monitor changes in the hypoxic fraction over the course of treatment is also of importance. Chemotherapy and radiotherapy are likely to influence the hypoxic fraction and therefore hypoxia imaging should be repeated on regular basis in order to correctly adapt the treatment. As discussed in chapter $\mathbf{2}$, voxel to voxel analysis of a $\left[{ }^{18} \mathrm{~F}\right]$ FAZA PET scan of a preclinical cervix tumor model showed a change in uptake after 4 and 10 fractions of $2.5 \mathrm{~Gy}$ or radiotherapy compared with the pretreatment scan (53). 
In addition the treatment effect of a pan-erbB tyrosine kinase inhibitors could be monitored over the course of treatment, revealing a decrease in the uptake of $\left[{ }^{18}\right.$ F]FAZA over days (57), which was also observed for Gefitinib, another tyrosine kinase inhibitor, while the control group remained hypoxic (58). In clinical studies monitoring of the hypoxic fraction was also performed during treatment. For head and neck cancer patients a decreased uptake of $\left[{ }^{18} \mathrm{~F}\right] \mathrm{FMISO}(42,59)$ or $\left[{ }^{18} \mathrm{~F}\right] \mathrm{FAZA}(60)$ was detected after treatment. However, for non-small cell lung cancer the observed changes over treatment were unpredictable or undetectable using $\left[{ }^{18} \mathrm{~F}\right] \mathrm{FMSO}(61,62)$, while $\left[{ }^{18} \mathrm{~F}\right] \mathrm{FAZA}$ did detect an elimination of the hypoxic fraction during treatment (11). This diversity in hypoxia detection over treatment suggests a difference in hypoxia persistence between different cancer sites and warrants further investigation. In addition, with this new knowledge complementary treatment can be given.

In Chapter 5 the pretreatment $\left[{ }^{18} \mathrm{~F}\right] \mathrm{HX} 4$ PET scan was shown to be a predictive factor in the treatment outcome of $\mathrm{TH}-302$. To induce altered oxygen concentrations in the tumor, oxygen modification was applied by either giving high oxygen concentrations (carbogen $\left[95 \% \mathrm{O}_{2}, 5 \% \mathrm{CO}_{2}\right]$ ) in combination with nicotinamide or low oxygen concentrations (7\% oxygen). This oxygen modification was applied already one day before treatment to assess the hypoxic fraction using $\left[{ }^{18} \mathrm{~F}\right] \mathrm{HX} 4$ imaging. The treatment effect of TH-302 was enhanced when the hypoxic fraction was increased while the absence of hypoxia completely abolished the TH-302 effect. In Chapter 3 it was already shown that the effect of oxygen modification can be observed by hypoxia PET imaging, although the approach was somewhat different than in Chapter 5. The hypoxia PET tracer was injected and accumulation was allowed under normal air conditions for 2.5 hours, before a PET scan was acquired. Only thereafter animals were exposed to modified oxygen concentrations and a second PET scan revealed that the uptake was modified compared to normal air breathing animals. It was demonstrated by others that oxygen breathing starting 2 minutes before $\left[{ }^{18} \mathrm{~F}\right] \mathrm{FAZA}$ injection was as good in modifying the tumor uptake as starting 1 hour up front (63). In another study performed in our lab using the same tumor model, the oxygenation status was modified 1 hour pre- and 2 hours post- treatment while the effect could be detected for an additional 2 hours on $\left.{ }^{18} \mathrm{~F}\right] \mathrm{HX} 4$ PET imaging (54), probably due to the retention of the tracer in the hypoxic cells. From this we can conclude that the detection of hypoxia using PET is sufficiently sensitive to the oxygen status of the tumor. Furthermore, it was shown that the treatment effect of $\mathrm{TH}-302$ is dependent on the amount of hypoxia present and this can be predicted by pretreatment hypoxia 
assessment. This demonstrates the strength of the interaction between the monitoring and targeting of hypoxia.

\section{Future perspectives}

Current clinical practice is shifting from a 'one size fits all' approach towards a more individualized treatment planning. This shift is stimulated by the increase in available knowledge, techniques and treatment options. The ability to detect tumor hypoxia, a main contributor to treatment outcome, has immensely evolved, together with the development of drugs specifically targeting those therapy-resistant areas. In this thesis two strategies to visualize hypoxia were assessed at preclinical level, accompanied by two strategies to target hypoxia. These strategies will contribute in the process towards personalized medicine; however, more steps need to be taken before this goal will be reached.

Studies have shown the prognostic and predictive value of hypoxia imaging or the ability to designate patients that will benefit from an additional therapy. In order to implement these data into the daily clinical practice, together with all the other available data of the medical and molecular field, a multifactorial decision support system is needed (64). This would allow the stratification of patients into a certain group and assign them to a suitable treatment. Over time, more information should be implemented in these databases, e.g. patient characteristics and additional treatments. However, to expand the databases, clinical studies are needed to test new drugs or treatment approaches available.

The implementation of new drugs in the clinic is a long and expensive process. Therefore 'window-of-opportunity' trials are recently being introduced $(65,66)$. Using this approach $\left[{ }^{18} \mathrm{~F}\right] \mathrm{HX} 4$ imaging may be used as biomarker of response, especially suitable in the context of testing new drugs for hypoxia targeting like TH-302. Upon patient inclusion, a baseline hypoxia PET scan should be performed followed by the administration of the hypoxia targeting drug. A post-treatment hypoxia PET scan can assess the effect of the single treatment on the hypoxic fraction and decide whether it is useful to continue the treatment. This will allow clinical testing of new treatment entities while being cost and patient efficient.

So far, clinical phase II trials have demonstrated the potential of $\mathrm{TH}-302$ in combination with chemotherapies, and phase III clinical trials are ongoing. Moreover, a 
phase I trial is starting soon to assess the effect of TH-302 in combination with radiotherapy. This illustrates the high potential of this hypoxia-activated drug. However, to make those clinical trials successful, pretreatment assessment of tumor hypoxia is necessary in order to select the patients that may benefit from this additional therapy.

In addition, monitoring and targeting of the hypoxia response protein CAIX also hold future possibilities, as demonstrated in this thesis. Although monitoring of CAIX activity may help to select patients that would benefit from additional CAIX inhibition treatment, there is no read-out for treatment efficacy yet. Upon the inhibition of the CAIX function, the protein is still available and therefore monitoring of the protein cannot be used. A so called 'biomarker of efficacy' might be of interest to select in an early phase of the treatment whether patients respond to the therapy. One possibility could be the detection of shed CAIX, detectable in the urine or blood plasma. Furthermore, modification on protein or genomic level induced by the CAIX inhibition which could be detected on patient tumor samples or circulating in the patient's blood, might serve as such a biomarker (67).

The development of cancer is in $65 \%$ of the tumor types caused by bad luck, due to random mutations induced in normal, non-cancerous stem cells during DNA replication. The complete prevention of cancer will therefore be impossible, even when the environmental factors and inherited predispositions do not contribute to this carcinogenic process (68). Knowledge about the tumor and its characteristics in an early stage of the disease will be of great importance to select the right treatment for each individual tumor. The detection of tumor hypoxia, as main contributor to therapy resistance, is proven to give important additional information. Further implementation of this technique and further development and validation of new anti-cancer treatments will improve the treatment of cancer patients and contribute to the aim of personalized medicine. 


\section{References}

1. Reischl G, Dorow DS, Cullinane C, Katsifis A, Roselt P, Binns D, et al. Imaging of tumor hypoxia with [124I]IAZA in comparison with [18F]FMISO and [18F]FAZA--first small animal PET results. J Pharm Pharm Sci. 2007;10:203-11.

2. Gronroos T, Bentzen L, Marjamaki P, Murata R, Horsman MR, Keiding S, et al. Comparison of the biodistribution of two hypoxia markers [18F]FETNIM and [18F]FMISO in an experimental mammary carcinoma. Eur J Nucl Med Mol Imaging. 2004;31:513-20.

3. Dubois L, Landuyt W, Cloetens L, Bol A, Bormans G, Haustermans K, et al. [18F]EF3 is not superior to [18F]FMISO for PET-based hypoxia evaluation as measured in a rat rhabdomyosarcoma tumour model. Eur J Nucl Med Mol Imaging. 2009;36:209-18.

4. Carlin S, Zhang H, Reese M, Ramos NN, Chen Q, Ricketts SA. A comparison of the imaging characteristics and microregional distribution of 4 hypoxia PET tracers. J Nucl Med. 2014;55:515-21.

5. Rasey JS, Koh WJ, Evans ML, Peterson LM, Lewellen TK, Graham MM, et al. Quantifying regional hypoxia in human tumors with positron emission tomography of [18F]fluoromisonidazole: a pretherapy study of 37 patients. Int J Radiat Oncol Biol Phys. 1996;36:417-28.

6. Szeto MD, Chakraborty G, Hadley J, Rockne R, Muzi M, Alvord EC, Jr., et al. Quantitative metrics of net proliferation and invasion link biological aggressiveness assessed by MRI with hypoxia assessed by FMISO-PET in newly diagnosed glioblastomas. Cancer research. 2009;69:4502-9.

7. Segard T, Robins PD, Yusoff IF, Ee H, Morandeau L, Campbell EM, et al. Detection of hypoxia with 18Ffluoromisonidazole (18F-FMISO) PET/CT in suspected or proven pancreatic cancer. Clinical nuclear medicine. 2013;38:1-6.

8. Loi S, Ngan SY, Hicks RJ, Mukesh B, Mitchell P, Michael M, et al. Oxaliplatin combined with infusional 5fluorouracil and concomitant radiotherapy in inoperable and metastatic rectal cancer: a phase I trial. $\mathrm{Br}$ J Cancer. 2005;92:655-61.

9. Lawrentschuk N, Poon AM, Foo SS, Putra LG, Murone C, Davis ID, et al. Assessing regional hypoxia in human renal tumours using $18 \mathrm{~F}$-fluoromisonidazole positron emission tomography. BJU Int. 2005;96:540-6.

10. Postema EJ, McEwan AJ, Riauka TA, Kumar P, Richmond DA, Abrams DN, et al. Initial results of hypoxia imaging using 1-alpha-D: -(5-deoxy-5-[18F]-fluoroarabinofuranosyl)-2-nitroimidazole ( 18F-FAZA). Eur J Nucl Med Mol Imaging. 2009;36:1565-73.

11. Trinkaus ME, Blum R, Rischin D, Callahan J, Bressel M, Segard T, et al. Imaging of hypoxia with 18F-FAZA PET in patients with locally advanced non-small cell lung cancer treated with definitive chemoradiotherapy. J Med Imaging Radiat Oncol. 2013;57:475-81.

12. Havelund BM, Holdgaard PC, Rafaelsen SR, Mortensen LS, Theil J, Bender D, et al. Tumour hypoxia imaging with $18 \mathrm{~F}$-fluoroazomycinarabinofuranoside PET/CT in patients with locally advanced rectal cancer. Nucl Med Commun. 2013;34:155-61.

13. Garcia-Parra R, Wood D, Shah RB, Siddiqui J, Hussain H, Park H, et al. Investigation on tumor hypoxia in resectable primary prostate cancer as demonstrated by 18F-FAZA PET/CT utilizing multimodality fusion techniques. Eur J Nucl Med Mol Imaging. 2011;38:1816-23.

14. Doss M, Zhang JJ, Belanger MJ, Stubbs JB, Hostetler ED, Alpaugh K, et al. Biodistribution and radiation dosimetry of the hypoxia marker $18 \mathrm{~F}-\mathrm{HX} 4$ in monkeys and humans determined by using whole-body PET/CT. Nucl Med Commun. 2010;31:1016-24.

15. van Loon J, Janssen MH, Ollers M, Aerts HJ, Dubois L, Hochstenbag M, et al. PET imaging of hypoxia using [18F]HX4: a phase I trial. Eur J Nucl Med Mol Imaging. 2010;37:1663-8. 
16. Chen L, Zhang Z, Kolb HC, Walsh JC, Zhang J, Guan Y. (1)(8)F-HX4 hypoxia imaging with PET/CT in head and neck cancer: a comparison with (1)(8)F-FMISO. Nucl Med Commun. 2012;33:1096-102.

17. Zegers $C M$, van Elmpt $W$, Wierts $R$, Reymen $B$, Sharifi $H$, Ollers $M C$, et al. Hypoxia imaging with [(1)(8)F]HX4 PET in NSCLC patients: defining optimal imaging parameters. Radiother Oncol. 2013;109:58-64.

18. Zegers CM, van Elmpt W, Reymen B, Even AJ, Troost EG, Ollers MC, et al. In Vivo Quantification of Hypoxic and Metabolic Status of NSCLC Tumors Using [18F]HX4 and [18F]FDG-PET/CT Imaging. Clin Cancer Res. 2014;20:6389-97.

19. Harris AL. Hypoxia--a key regulatory factor in tumour growth. Nat Rev Cancer. 2002;2:38-47.

20. Generali D, Berruti A, Brizzi MP, Campo L, Bonardi S, Wigfield S, et al. Hypoxia-inducible factor-1alpha expression predicts a poor response to primary chemoendocrine therapy and disease-free survival in primary human breast cancer. Clin Cancer Res. 2006;12:4562-8.

21. Shin KH, Diaz-Gonzalez JA, Russell J, Chen Q, Burgman P, Li XF, et al. Detecting changes in tumor hypoxia with carbonic anhydrase IX and pimonidazole. Cancer Biol Ther. 2007;6:70-5.

22. Rademakers SE, Lok J, van der Kogel AJ, Bussink J, Kaanders JH. Metabolic markers in relation to hypoxia; staining patterns and colocalization of pimonidazole, HIF-1alpha, CAIX, LDH-5, GLUT-1, MCT1 and MCT4. BMC Cancer. 2011;11:167.

23. Nordsmark M, Eriksen JG, Gebski V, Alsner J, Horsman MR, Overgaard J. Differential risk assessments from five hypoxia specific assays: The basis for biologically adapted individualized radiotherapy in advanced head and neck cancer patients. Radiother Oncol. 2007;83:389-97.

24. Dubois L, Douma K, Supuran CT, Chiu RK, van Zandvoort MA, Pastorekova S, et al. Imaging the hypoxia surrogate marker CA IX requires expression and catalytic activity for binding fluorescent sulfonamide inhibitors. Radiother Oncol. 2007;83:367-73.

25. Tafreshi NK, Lloyd MC, Bui MM, Gillies RJ, Morse DL. Carbonic anhydrase IX as an imaging and therapeutic target for tumors and metastases. Subcell Biochem. 2014;75:221-54.

26. Liu Z, Pourghiasian M, Radtke MA, Lau J, Pan J, Dias GM, et al. An organotrifluoroborate for broadly applicable one-step 18F-labeling. Angew Chem Int Ed Engl. 2014;53:11876-80.

27. Honarvar H, Garousi J, Gunneriusson E, Hoiden-Guthenberg I, Altai M, Widstrom C, et al. Imaging of CAIX-expressing xenografts in vivo using 99mTc-HEHEHE-ZCAIX:1 Affibody molecule. International journal of oncology. 2015;46:513-20.

28. Chawla SP, Cranmer LD, Van Tine BA, Reed DR, Okuno SH, Butrynski JE, et al. Phase II study of the safety and antitumor activity of the hypoxia-activated prodrug TH-302 in combination with doxorubicin in patients with advanced soft tissue sarcoma. J Clin Oncol. 2014;32:3299-306.

29. Liu Q, Sun JD, Wang J, Ahluwalia D, Baker AF, Cranmer LD, et al. TH-302, a hypoxia-activated prodrug with broad in vivo preclinical combination therapy efficacy: optimization of dosing regimens and schedules. Cancer Chemother Pharmacol. 2012;69:1487-98.

30. Sun JD, Liu $Q$, Wang J, Ahluwalia D, Ferraro $D$, Wang $Y$, et al. Selective tumor hypoxia targeting by hypoxia-activated prodrug TH-302 inhibits tumor growth in preclinical models of cancer. Clin Cancer Res. 2012;18:758-70.

31. Reddy SB, Williamson SK. Tirapazamine: a novel agent targeting hypoxic tumor cells. Expert Opin Investig Drugs. 2009;18:77-87.

32. Borad MJ, Reddy SG, Bahary N, Uronis HE, Sigal D, Cohn AL, et al. Randomized Phase II Trial of Gemcitabine Plus TH-302 Versus Gemcitabine in Patients With Advanced Pancreatic Cancer. J Clin Oncol. 2014.

33. Svastova E, Hulikova A, Rafajova M, Zat'ovicova M, Gibadulinova A, Casini A, et al. Hypoxia activates the capacity of tumor-associated carbonic anhydrase IX to acidify extracellular pH. FEBS Lett. 2004;577:439-45 
34. Swietach P, Patiar S, Supuran CT, Harris AL, Vaughan-Jones RD. The role of carbonic anhydrase 9 in regulating extracellular and intracellular ph in three-dimensional tumor cell growths. The Journal of biological chemistry. 2009;284:20299-310.

35. Webb BA, Chimenti M, Jacobson MP, Barber DL. Dysregulated pH: a perfect storm for cancer progression. Nat Rev Cancer. 2011;11:671-7.

36. Overgaard J, Overgaard M, Nielsen OS, Pedersen AK, Timothy AR. A comparative investigation of nimorazole and misonidazole as hypoxic radiosensitizers in a $\mathrm{C} 3 \mathrm{H}$ mammary carcinoma in vivo. $\mathrm{Br} \mathrm{J}$ Cancer. 1982;46:904-11.

37. Cianchi F, Vinci MC, Supuran CT, Peruzzi B, De Giuli P, Fasolis G, et al. Selective inhibition of carbonic anhydrase IX decreases cell proliferation and induces ceramide-mediated apoptosis in human cancer cells. J Pharmacol Exp Ther. 2010;334:710-9.

38. Ditte P, Dequiedt F, Svastova E, Hulikova A, Ohradanova-Repic A, Zatovicova M, et al. Phosphorylation of carbonic anhydrase IX controls its ability to mediate extracellular acidification in hypoxic tumors. Cancer Res. 2011;71:7558-67.

39. Lock FE, McDonald PC, Lou Y, Serrano I, Chafe SC, Ostlund C, et al. Targeting carbonic anhydrase IX depletes breast cancer stem cells within the hypoxic niche. Oncogene. 2013;32:5210-9.

40. Wouters BG, Koritzinsky M. Hypoxia signalling through mTOR and the unfolded protein response in cancer. Nat Rev Cancer. 2008;8:851-64.

41. Svastova E, Witarski W, Csaderova L, Kosik I, Skvarkova L, Hulikova A, et al. Carbonic anhydrase IX interacts with bicarbonate transporters in lamellipodia and increases cell migration via its catalytic domain. J Biol Chem. 2012;287:3392-402.

42. Lee N, Nehmeh S, Schoder H, Fury M, Chan K, Ling CC, et al. Prospective trial incorporating pre-/midtreatment [18F]-misonidazole positron emission tomography for head-and-neck cancer patients undergoing concurrent chemoradiotherapy. International journal of radiation oncology, biology, physics. 2009;75:101-8.

43. Rajendran JG, Schwartz DL, O'Sullivan J, Peterson LM, Ng P, Scharnhorst J, et al. Tumor hypoxia imaging with [F-18] fluoromisonidazole positron emission tomography in head and neck cancer. Clinical cancer research : an official journal of the American Association for Cancer Research. 2006;12:5435-41.

44. Sato J, Kitagawa Y, Yamazaki Y, Hata H, Asaka T, Miyakoshi M, et al. Advantage of FMISO-PET over FDGPET for predicting histological response to preoperative chemotherapy in patients with oral squamous cell carcinoma. Eur J Nucl Med Mol Imaging. 2014.

45. Cher LM, Murone C, Lawrentschuk N, Ramdave S, Papenfuss A, Hannah A, et al. Correlation of hypoxic cell fraction and angiogenesis with glucose metabolic rate in gliomas using $18 \mathrm{~F}$-fluoromisonidazole, 18F-FDG PET, and immunohistochemical studies. J Nucl Med. 2006;47:410-8.

46. Kawai N, Lin W, Cao WD, Ogawa D, Miyake K, Haba R, et al. Correlation between (18)Ffluoromisonidazole PET and expression of HIF-1alpha and VEGF in newly diagnosed and recurrent malignant gliomas. Eur J Nucl Med Mol Imaging. 2014;41:1870-8.

47. Swanson KR, Chakraborty G, Wang CH, Rockne R, Harpold HL, Muzi M, et al. Complementary but distinct roles for MRI and 18F-fluoromisonidazole PET in the assessment of human glioblastomas. J Nucl Med. 2009;50:36-44.

48. Cheng J, Lei L, Xu J, Sun Y, Zhang Y, Wang X, et al. 18F-fluoromisonidazole PET/CT: a potential tool for predicting primary endocrine therapy resistance in breast cancer. J Nucl Med. 2013;54:333-40.

49. Bussink J, van Herpen CM, Kaanders JH, Oyen WJ. PET-CT for response assessment and treatment adaptation in head and neck cancer. Lancet Oncol. 2010;11:661-9.

50. Dubois L, Landuyt W, Haustermans K, Dupont P, Bormans G, Vermaelen P, et al. Evaluation of hypoxia in an experimental rat tumour model by [(18)F]fluoromisonidazole PET and immunohistochemistry. Br J Cancer. 2004;91:1947-54. 
51. Troost EG, Laverman P, Kaanders JH, Philippens M, Lok J, Oyen WJ, et al. Imaging hypoxia after oxygenation-modification: comparing [18F]FMISO autoradiography with pimonidazole immunohistochemistry in human xenograft tumors. Radiother Oncol. 2006;80:157-64.

52. Busk M, Horsman MR, Jakobsen S, Keiding S, van der Kogel AJ, Bussink J, et al. Imaging hypoxia in xenografted and murine tumors with 18F-fluoroazomycin arabinoside: a comparative study involving microPET, autoradiography, PO2-polarography, and fluorescence microscopy. Int J Radiat Oncol Biol Phys. 2008;70:1202-12.

53. Busk M, Mortensen LS, Nordsmark M, Overgaard J, Jakobsen S, Hansen KV, et al. PET hypoxia imaging with FAZA: reproducibility at baseline and during fractionated radiotherapy in tumour-bearing mice. Eur J Nucl Med Mol Imaging. 2013;40:186-97.

54. Dubois LJ, Lieuwes NG, Janssen MH, Peeters WJ, Windhorst AD, Walsh JC, et al. Preclinical evaluation and validation of [18F]HX4, a promising hypoxia marker for PET imaging. Proc Natl Acad Sci U S A. 2011;108:14620-5.

55. Hendrickson K, Phillips M, Smith W, Peterson L, Krohn K, Rajendran J. Hypoxia imaging with [F-18] FMISO-PET in head and neck cancer: potential for guiding intensity modulated radiation therapy in overcoming hypoxia-induced treatment resistance. Radiother Oncol. 2011;101:369-75.

56. Thorwarth D, Eschmann SM, Paulsen F, Alber M. Hypoxia dose painting by numbers: a planning study. Int J Radiat Oncol Biol Phys. 2007;68:291-300.

57. Dorow DS, Cullinane C, Conus N, Roselt P, Binns D, McCarthy TJ, et al. Multi-tracer small animal PET imaging of the tumour response to the novel pan-Erb-B inhibitor $\mathrm{Cl}-1033$. Eur J Nucl Med Mol Imaging. 2006;33:441-52.

58. Solomon B, Binns D, Roselt P, Weibe LI, McArthur GA, Cullinane C, et al. Modulation of intratumoral hypoxia by the epidermal growth factor receptor inhibitor gefitinib detected using small animal PET imaging. Mol Cancer Ther. 2005;4:1417-22.

59. Yamane T, Kikuchi M, Shinohara S, Senda M. Reduction of [(18)F]fluoromisonidazole uptake after neoadjuvant chemotherapy for head and neck squamous cell carcinoma. Mol Imaging Biol. 2011;13:227-31.

60. Servagi-Vernat S, Differding S, Hanin FX, Labar D, Bol A, Lee JA, et al. A prospective clinical study of (1)(8)F-FAZA PET-CT hypoxia imaging in head and neck squamous cell carcinoma before and during radiation therapy. Eur J Nucl Med Mol Imaging. 2014;41:1544-52.

61. Koh WJ, Bergman KS, Rasey JS, Peterson LM, Evans ML, Graham MM, et al. Evaluation of oxygenation status during fractionated radiotherapy in human nonsmall cell lung cancers using [F18]fluoromisonidazole positron emission tomography. International journal of radiation oncology, biology, physics. 1995;33:391-8.

62. Vera P, Bohn P, Edet-Sanson A, Salles A, Hapdey S, Gardin I, et al. Simultaneous positron emission tomography (PET) assessment of metabolism with (1)(8)F-fluoro-2-deoxy-d-glucose (FDG), proliferation with (1)(8)F-fluoro-thymidine (FLT), and hypoxia with (1)(8)fluoro-misonidazole (F-miso) before and during radiotherapy in patients with non-small-cell lung cancer (NSCLC): a pilot study. Radiotherapy and oncology : journal of the European Society for Therapeutic Radiology and Oncology. 2011;98:10916.

63. Maier FC, Kneilling M, Reischl G, Cay F, Bukala D, Schmid A, et al. Significant impact of different oxygen breathing conditions on noninvasive in vivo tumor-hypoxia imaging using [(1)(8)F]-fluoroazomycinarabino-furanoside ([(1)(8)F]FAZA). Radiat Oncol. 2011;6:165.

64. Lambin P, van Stiphout RG, Starmans MH, Rios-Velazquez E, Nalbantov G, Aerts HJ, et al. Predicting outcomes in radiation oncology--multifactorial decision support systems. Nat Rev Clin Oncol. 2013;10:27-40.

65. Fass L. Imaging and cancer: a review. Mol Oncol. 2008;2:115-52. 
66. Orloff J, Douglas F, Pinheiro J, Levinson S, Branson M, Chaturvedi P, et al. The future of drug development: advancing clinical trial design. Nat Rev Drug Discov. 2009;8:949-57.

67. Neal JW, Gainor JF, Shaw AT. Developing biomarker-specific end points in lung cancer clinical trials. Nat Rev Clin Oncol. 2014.

68. Tomasetti C, Vogelstein B. Cancer etiology. Variation in cancer risk among tissues can be explained by the number of stem cell divisions. Science. 2015;347:78-81. 
Summary 
Solid tumors are often characterized with regions of low oxygen concentrations, hypoxia, caused by chaotic blood vessel development and impaired perfusion. Those regions are known to be less sensitive to conventional anticancer treatments like chemotherapy and radiotherapy. Knowledge about the presence and location of hypoxic tumor regions can be obtained by performing a positron emission tomography (PET) scan combined with a computed tomography (CT) scan for anatomical information. The detection of hypoxia can be done in a direct manner using PET tracers that accumulate in hypoxic regions. Another approach is to use a surrogate hypoxia marker, a tracer that interacts with proteins that are upregulated in a hypoxiadependent manner like carbonic anhydrase IX (CAIX). Since the treatment response to conventional anticancer therapies is negatively influenced by the presence of tumor hypoxia, there is a need for drugs specifically targeting those hypoxic regions. In line with hypoxia imaging, therapies against hypoxic cells can be either directed towards hypoxic cells themselves as well as directed to the hypoxia responsive proteins.

The aim of this thesis is to use the theranostic approach, combining imaging and therapy, to conduct preclinical studies in line with the concept of personalized medicine. The first part focused on the noninvasive visualization of tumor hypoxia, while the objective of the second part was to target tumor hypoxia, where possible guided by hypoxia imaging, with the aim to improve therapy outcome.

\section{Part I: Noninvasive imaging of the hypoxic tumor microenvironment}

Numerous 2-nitroimidazole based PET tracers have been developed for the noninvasive detection of tumor hypoxia. After initial validation in preclinical setting, several tracers were evaluated in clinical trials. Chapter 2 gives an overview of the tracers that have demonstrated clinical potential. Both preclinical and clinical studies are discussed with regard to validation, quantification, pharmacokinetic analysis, applications like prognostic / predictive value and monitoring of tumor hypoxia during treatment and hypoxia targeting. These studies confirm the value of noninvasive PET imaging in the monitoring of hypoxia. However, more clinical trials are needed before hypoxia PET tracers will be incorporated in daily clinical practice. So far, no direct comparison was performed between multiple hypoxia PET tracers. Most studies focus on one single tracer or a comparison with polarographic oxygen electrodes or $\left[{ }^{18} \mathrm{~F}\right] \mathrm{FMISO}$ solely. In chapter 3 , three clinically available hypoxia PET tracers were compared within one preclinical tumor model. For $\left[{ }^{18} \mathrm{~F}\right] \mathrm{FMISO},\left[{ }^{18} \mathrm{~F}\right] \mathrm{FAZA}$ and $\left[{ }^{18} \mathrm{~F}\right] \mathrm{HX} 4$ the optimal imaging time point was determined together with the reproducibility of the tracer uptake over 48 hours. Sensitivity to oxygen modifications was also 
investigated to characterize the differences between the tracers. This study indicates that all investigated tracers show different characteristics.

Another approach to visualize tumor hypoxia is by imaging the hypoxia responsive protein CAIX. The transcription of this protein is upregulated via 'hypoxia-inducible factor' (HIF) which binds to the 'hypoxia-response element' (HRE) on the CA9 gene. Therefore the expression of CAIX is increased upon hypoxia and can be seen as a surrogate marker of hypoxia. For the detection of CAIX [ $\left.{ }^{18} \mathrm{~F}\right] \mathrm{VM} 4-037$ was developed and the clinical safety of this tracer was already demonstrated in a phase I study. The uptake and CAIX specificity of $\left[{ }^{18}\right.$ F]VM4-037 was studied in chapter 4 using two CAIX expressing preclinical tumor models. No specific uptake in the tumors could be detected, indicating that VM4-037 is not suitable for CAIX visualization.

\section{Part II: Targeting of the hypoxic tumor regions and the hypoxia response.}

Specific targeting of the hypoxic tumor regions was studied in the second part of the thesis. Drugs have been developed that are non-toxic under normal oxygen concentrations and are converted to a cytotoxic variant under hypoxia. One of these so-called 'hypoxia-activated prodrugs' is TH-302. The effect of TH-302 monotherapy and its effect in combination with chemotherapy showed promising results. In chapter 5 the combination of TH-302 and radiotherapy was investigated for the first time. Two preclinical tumor models were treated with the combination of radiotherapy and $\mathrm{TH}-$ 302. TH-302 was demonstrated to decrease the hypoxic fraction and decrease the tumor growth, while the combination treatment of TH-302 and radiotherapy had an enhanced effect and further reduced the tumor growth. Furthermore, the hypoxic fraction before treatment as detected by $\left[{ }^{18} \mathrm{~F}\right] \mathrm{HX} 4$ hypoxia imaging was associated with the treatment outcome. A clinical trial will be started to further study the effects of TH302 in combination with radiotherapy.

In chapter 6 and 7 the potential of small molecule inhibitors of CAIX in improving radiotherapy outcome was studied. The expression of CAIX is mostly tumor specific and upregulated under hypoxic conditions. Furthermore, CAIX is known to have a role in the cellular $\mathrm{pH}$ balance; it maintains a stable intracellular $\mathrm{pH}$ while it acidifies the extracellular environment contributing to more aggressive cellular behavior. An indanesulfonamide specifically targeting CAIX function was studied in chapter 6 in combination with radiotherapy and showed a reduction in the tumor growth. The effect of a dual targeting compound, DH348, was assessed in chapter 7. The novelty of this compound lies within its two sided function; the sulfonamide part is directed 
against the active site of CAIX while the 5-nitroimidazole part functions as a radiosensitizer in hypoxic areas. The effect of the dual targeting drug DH348 was demonstrated to be more effective in combination with radiotherapy than the single CAIX targeting drug.

In conclusion, this thesis confirms the potential of hypoxia PET tracer to monitor tumor hypoxia and further characterizes the different tracers on a preclinical level. Furthermore, specific targeting of hypoxia using the hypoxia activated prodrug $\mathrm{TH}-302$ or inhibitors of CAIX has been shown to radio-sensitize tumors and improve the outcome in a combined treatment. Most important, the ability of $\left[{ }^{18} \mathrm{~F}\right] \mathrm{HX} 4$ to monitor tumor hypoxia over TH-302 treatment has been demonstrated as well as the association between pretreatment hypoxia imaging and therapy outcome. This theranostic approach demonstrates the strength of combining hypoxia imaging and targeting. 


\section{Samenvatting}


Een veel voorkomend kenmerk van solide tumoren is het gebrek aan zuurstof in sommige gebieden. Deze zuurstof arme gebieden worden ook wel hypoxische gebieden genoemd en worden veroorzaakt door chaotisch gevormde bloedvaten waardoor zowel de perfusie als de diffusie van zuurstof in de tumor wordt beperkt. Het is bekend dat deze hypoxische gebieden minder gevoelig zijn voor kanker behandelingen zoals chemotherapie en bestraling / radiotherapie. Een PET scan kan informatie geven over de aanwezigheid van tumor hypoxie en wordt vaak gecombineerd met een CT scan voor anatomische informatie. Een PET scan maakt gebruik van radioactief gelabelde tracers die worden geïnjecteerd in een patiënt en die specifiek accumuleren in zuurstof arme gebieden waardoor de aanwezigheid van hypoxie kan worden gedetecteerd. Een andere manier om hypoxie te detecteren is door middel van een surrogaat hypoxie merker; een tracer die bindt aan een eiwit dat wordt gereguleerd door hypoxie en enkel onder lage zuurstof condities aanwezig is. Een eiwit waarvan de expressie op deze manier wordt geregeld is koolzuur anhydrase ofwel carbonic anhydrase IX (CAIX).

Het succes van de klassieke kanker behandelingen wordt negatief beïnvloed door de aanwezigheid van hypoxie en daarom zijn er nieuwe medicijnen nodig die specifiek gericht zijn tegen deze gebieden. Net als bij hypoxie beeldvorming kan de anti-hypoxie therapie worden gericht direct tegen de hypoxie zelf, maar ook tegen de eiwitten die worden gereguleerd onder hypoxie.

Het doel van dit proefschrift is om door middel van een theranostische benadering, het combineren van beeldvorming/diagnostiek en therapie, studies uit te voeren volgens het concept van gepersonaliseerde geneeskunde. Het eerste deel van het proefschrift is gericht op de niet-invasieve beeldvorming van tumor hypoxie, terwijl het tweede deel van zich richt op nieuwe therapieën tegen tumor hypoxie, waar mogelijk gecombineerd met hypoxie beeldvorming.

\section{Deel 1: Niet-invasieve beeldvorming van de hypoxische tumor omgeving}

Verschillende PET tracers zijn gebaseerd op een 2-nitroimidazole scheikundige groep en zijn ontwikkeld voor de niet-invasieve detectie van tumor hypoxie. De eerste validatie van deze tracers gebeurt in preklinische modellen voordat ze verder worden geëvalueerd in klinische studies. In hoofdstuk 2 wordt een overzicht gegeven van tracers met een bewezen klinische potentie. Hierin worden zowel preklinische als klinische studies beschreven met betrekking tot validatie, kwantificatie, farmacokinetische analyses, toepassingen zoals prognostische en predicatieve waarde en het 
opvolgen van tumor hypoxie tijdens de conventionele anti-kanker behandeling of specifieke hypoxie gerichte therapie. Al deze studies tonen de toegevoegde waarde van niet-invasieve PET beeldvorming in het opvolgen van tumor hypoxie. Er zijn echter meer klinische studies nodig voordat hypoxie PET beeldvorming daadwerkelijk zal worden toegepast in de dagelijkse praktijk. Tot op heden zijn er geen studies uitgevoerd die een directe vergelijking maken tussen verschillende hypoxie PET tracers. De meeste studies bestuderen één enkele tracer en vergelijken deze enkel met invasieve technieken of met de eerst ontwikkelde hypoxie PET tracer $\left[{ }^{18} \mathrm{~F}\right] \mathrm{FMISO}$. In hoofdstuk 3 worden drie klinisch beschikbare hypoxie PET tracers vergeleken binnen één preklinisch tumor model. Voor $\left[{ }^{18} \mathrm{~F}\right] \mathrm{FMISO},\left[{ }^{18} \mathrm{~F}\right] \mathrm{FAZA}$ en $\left[{ }^{18} \mathrm{~F}\right] \mathrm{HX} 4$ werd het optimale tijdspunt voor beeldvorming bepaald samen met de reproduceerbaarheid van de tracer opname over een tijdsinterval van 48 uur. Daarnaast werd de gevoeligheid aan veranderingen in zuurstof concentratie bepaald voor de verschillende tracers. Deze studie laat zien dat de drie bestudeerde tracers verschillende karakteristieken hebben.

Een andere manier om tumor hypoxie in beeld te brengen is door middel van beeldvorming van de moleculaire reactie ten gevolge van hypoxie en CAIX is een van de eiwitten die hiervoor geschikt is. De transcriptie van dit eiwit wordt gereguleerd door de 'hypoxie-induceerbare factor' (HIF) waardoor CAIX verhoogd tot expressie komt onder hypoxische condities en kan worden gezien als een surrogaat marker voor hypoxie. Voor de detectie van CAIX is een PET tracer ontwikkeld, [ $\left.{ }^{18} \mathrm{~F}\right] \mathrm{VM} 4-037$, waarvan in een klinische fase I studie de veiligheid voor patiënten is aangetoond. Hoofdstuk 4 bestudeerd in welke mate $\left[{ }^{18} \mathrm{~F}\right] \mathrm{VM} 4-037$ specifiek is voor CAIX door gebruik te maken van twee CAIX positieve preklinische tumor modellen. In de tumoren wordt echter geen specifieke opname van de tracer gedetecteerd en hierdoor lijkt $\left[{ }^{18} \mathrm{~F}\right] \mathrm{VM} 4-037$ niet geschikt voor de visualisatie van CAIX.

\section{Deel II: Therapieën tegen de hypoxische tumor gebieden}

Het tweede deel van dit proefschrift onderzoekt therapieën die specifiek gericht zijn tegen de hypoxische gebieden in de tumor. Er bestaan medicijnen die niet werkzaam zijn onder normale zuurstof concentraties en die worden geactiveerd onder hypoxie waardoor ze specifiek deze cellen doden. TH-302 is een van deze zogenaamde 'hypoxie geactiveerde pro-drugs'. De werking van TH-302 als monotherapie en het effect in combinatie met chemotherapie is al aangetoond en lijkt veelbelovend. In hoofdstuk 5 wordt de combinatie van TH-302 en radiotherapie voor de eerste keer bestudeerd. Twee preklinische modellen worden behandeld met de combinatie therapie van TH- 
302 en radiotherapie. Behandeling met enkel TH-302 laat een afname in de hoeveelheid hypoxie zien en een vertraging in de tumor groei, terwijl de combinatie behandeling van TH-302 en radiotherapie de tumor groei nog verder vertraagd. Door middel van $\left[{ }^{18} \mathrm{~F}\right] \mathrm{HX} 4$ hypoxie PET beeldvorming wordt de hypoxische fractie bepaald voor de start van de behandeling en deze is geassocieerd met het effect van TH-302 behandeling. Een klinische studie wordt gestart om het effect van TH-302 in combinatie met radiotherapie verder te bestuderen.

In hoofdstuk 6 en 7 wordt onderzocht of CAIX geremd kan worden door middel van een klein molecuul dat bindt aan het actieve deel van CAIX en of hiermee het effect van radiotherapie kan worden vergroot. CAIX wordt voornamelijk in de hypoxische tumor gebieden tot expressie gebracht waar het een rol heeft in de $\mathrm{pH}$ balans van de cel; het draagt bij aan een stabiele intracellulaire $\mathrm{pH}$ en gaat samen met verzuring van de extracellulaire omgeving waardoor een cel agressiever gedrag gaat vertonen. Hoofdstuk 6 bestudeerd een indanesulfonamide dat specifiek gericht is tegen CAIX en in combinatie met radiotherapie laat dit een afname van de tumor groei zien. In hoofdstuk 7 wordt DH348, een samengesteld molecuul, onderzocht. Het vernieuwende aspect van dit molecuul is de tweezijdige functie; het sulfamide deel is gericht tegen het actieve deel van CAIX terwijl het 5-nitroimidazole gedeelte de hypoxische gebieden gevoeliger maakt voor bestraling. Wanneer DH348 wordt gecombineerd met radiotherapie is het effect hiervan groter dan van een molecuul dat enkel tegen CAIX gericht is.

Concluderend laat dit proefschrift zien dat PET beeldvorming de potentie heeft om de mate van hypoxie in de tumor te bepalen en werden verschillende karakteristieken van enkele hypoxie tracer bestudeerd op preklinisch niveau. Daarnaast werd het effect van medicijnen, specifiek gericht tegen hypoxie, onderzocht door middel van twee benaderingen; TH-302 wat wordt geactiveerd onder lage zuurstof concentraties en remmers van het hypoxie gereguleerde eiwit CAIX. Beide therapieën laten zien dat de tumor gevoeliger wordt voor bestraling wat zorgt voor een betere uitkomst. Bovendien is $\left[{ }^{18} \mathrm{~F}\right] \mathrm{HX} 4$ in staat tot hypoxie beeldvorming gedurende de $\mathrm{TH}-302$ behandeling en is er een associatie tussen de hypoxische fractie, gedetecteerd voor de behandeling, en het effect van behandeling. Deze theranostische benadering bevestigt de kracht van het combineren van hypoxie beeldvorming en het specifiek richten van een behandeling tegen hypoxie. 


\section{Valorization addendum}


In this thesis the scientific relevance of imaging and specific targeting of the hypoxic tumor has been demonstrated. However, to make this knowledge of value for the general society, it has to be converted into a product or process that can be applied and results in a benefit for the community. Cancer is one of the main causes of death worldwide and although the anticancer treatments are improving, there is no such thing as a 'golden bullet'. It has been realized that every patient is unique and with that every tumor as well. Therefore, treatment has shifted from a 'one size fits all' approach to a more individualized treatment plan in which the anticancer therapy is adapted to the specific characteristics of the cancer. In this chapter, the knowledge obtained in this thesis will be placed in perspective of the valorization process, discuss its value for the general society, and the potential role in the future of cancer treatment.

\section{Clinical relevance}

Since the treatment of cancer patients is more and more moving into a personalized treatment, knowledge about the tumor and its heterogeneity is of great importance in selecting the most optimal treatment strategy. Conventional anti-cancer therapies are known to be less effective when tumors have regions of low oxygen concentrations, also known as hypoxia, and the prognosis of these patients is worse. Therefore there is a need for the detection of hypoxia and furthermore, additional treatment options for hypoxia targeting are needed to improve the prognosis. The information about the presence of hypoxia can be obtained by tumor biopsies. However, this technique is invasive and the information is based on a small piece of the tumor, not necessarily representing the whole tumor. In this thesis the topic of hypoxia PET imaging is discussed and investigated (chapter 2, 3, 4 and 5). The advantage of this technique is that it is noninvasive, repeatable, and does represent the whole tumor, thereby providing important information for the selection of patients suitable for a specific therapy. Hereby the treatment can better be stratified to the suited patients. Studies in this thesis validate three hypoxia PET tracers on several characteristics (chapter 3 ) and show an association between pretreatment monitoring of tumor hypoxia and therapy outcome (chapter 5). Although this thesis involves mostly preclinical work which cannot one to one be translated to the human situation, these kind of studies are needed to provide a first indication before moving to patients. For the hypoxia tracer $\left[{ }^{18} \mathrm{~F}\right] \mathrm{HX} 4$, which showed very promising results on preclinical level, promising data have been obtained from first clinical trials, underscoring the value of preclinical research. 
This thesis also investigates potential new drugs that could sensitize tumors to radiotherapy. Since conventional anticancer therapies are less effective in hypoxic regions, specific drugs targeting these areas could improve the treatment outcome. In chapter 5 TH-302, a drug specifically activated in hypoxic regions, is investigated. The efficacy of this drug in combination with chemotherapy was already demonstrated and is currently under investigation in clinical studies. In this thesis the combination of TH302 and radiotherapy is studied for the first time and shows promising results, and based on this a first clinical trial will be started. Patients with hypoxic tumors could benefit from this additional hypoxia targeting treatment to the standard treatment they receive.

From an economically perspective the application of hypoxia PET imaging would be of interest. When patients can be stratified into being hypoxic or not, their treatment plan can be adapted. Instead of randomly assigning patients to a certain treatment group, more specific treatment plans can be applied, resulting in a more cost efficient way of treatment. This also underscores the importance of good hypoxia targeting drugs (as investigated in chapter 5, 6 and 7), which can improve treatment outcome.

\section{Gain for society}

The translation of the scientific findings discussed in this thesis towards the daily clinical practice would result in a benefit for both doctor and cancer patient. First of all would the doctor be able to make better decisions in treatment planning and have a wider range of additional drugs available. A hypoxia PET scan would give information on the presence and amount of hypoxia, and even more value could be added when this monitoring of hypoxia could be extended from pretreatment monitoring only, to evaluating hypoxia over treatment. A first association between pretreatment hypoxia imaging and treatment outcome of a specific hypoxia targeting drug is demonstrated in this thesis, together with monitoring of the treatment effect after hypoxia targeting therapy (chapter 5). Furthermore when there is a broad range of drugs available, each specifically targeting a characteristic of a tumor, this would increase the tumor control probability. With this information on tumor hypoxia and with the specific targeting drugs present, the doctor could better help the patient. Therefore, not only the doctor will gain from these scientific findings, but also the patient will benefit, although the last mentioned will most likely not be aware of all the research that underlies the treatment. 


\section{Improve in health care}

The theranostic approach of this thesis could potentially contribute to an improved health care. Imaging of tumor characteristics like hypoxia has been demonstrated in this thesis (chapter 2, 3 and 5). Although this is still a very costly tool, it might be able to select patients for a specific therapy and monitor at early stage if the treatment is effective. By applying this noninvasive technique, patients are not exposed to situations that might interfere with their general health condition. One disadvantage of this technique is the scanning procedure, which involves an intravenous injection of a radioactive labeled tracer, followed by a scan 2 to 3 hours later in which the patient has to lay still. However, the obtained information can better select patients for a certain treatment group which might prevent patients from randomly being exposed to one treatment that is not suitable for them, causing switching between multiple treatments. The further development of specific hypoxia targeting drugs also contributes to this. Monitoring of tumor hypoxia is only relevant when specific treatments or drugs for these patients exist to improve their outcome. In this thesis two approaches have been investigated; direct targeting of the hypoxic regions by $\mathrm{TH}$ 302 (chapter 5) and indirect hypoxia targeting via CAIX (chapter 6\&7).

\section{Novelty of the concept}

The concept of visualizing tumor hypoxia is not new. Several techniques exist that have the potency to visualize the human body, the tumor in general and specific characteristics of the humor. For the detection of tumor hypoxia using PET, multiple tracers have been developed and validated in preclinical and clinical studies (as reviewed in chapter 2). However, the study described in chapter 3 is the first to compare three clinically used hypoxia PET tracers within one tumor model. Imaging of the hypoxia response by using small molecules to target CAIX (chapter 4) is also a known concept although so far this has only been successful using antibodies or relatively large molecules.

Since conventional anticancer treatments are impaired by certain tumor characteristics like hypoxia, several drugs have been developed that specifically target those therapy resistant regions. The aim is to sensitize tumors to the conventional chemo- or radiotherapy and improve the therapeutic outcome. For $\mathrm{TH}-302$, a hypoxia-activated cytotoxin, the combination with chemotherapy was already investigated in clinical trials and showed promising results. Therefore the novelty of chapter 5 cannot be found in the function of $\mathrm{TH}-302$, but in the combination with radiotherapy. The 
concept of sensitizing tumors by eradicating the hypoxic fraction has been studied before using other hypoxia-activated produgs, but was so far not effective. In our study, TH-302 showed to be a potent drug with its therapeutic effect being dependent on the amount of hypoxia.

The idea to target CAIX as a therapeutic target has also been studied before. However, the approach of dual targeting, as described in chapter 7, is a novel concept. The drug investigated in this study is directed against both hypoxia as well as CAIX and with that aims to increase the specificity of the targeting. For this group of compounds a patent has been obtained, however, the efficacy of this class of drugs needs to be investigated in more detail before it can be used in the clinical practice.

\section{Road to the market}

The research described in this thesis does have a potential clinical relevance with a gain for society and could eventually lead to an improved health care. However, there is still a long way to go before this will all be implemented. Hypoxia imaging is already applied in clinical trials and has shown some promising first results. Although some first steps are taken, more, mainly clinical, studies are needed to really prove the additive value of hypoxia imaging before it will be used in daily clinical practice.

Several steps need to be taken before the concept of CAIX imaging can be applied in clinical practice. First an imaging tracer needs to be developed and tested in preclinical setting before first clinical trials, toxicology studies, can be performed. The strength of this concept could really be underscored if also a specific CAIX targeting drug would be available that has a clear demonstrated effect on treatment outcome. The combination of the two, imaging and targeting of CAIX, could be very interesting, but still has a long way to come.

For the bioreductive drug TH-302 clinical trials are already in clinical phase III, however, this is in combination with chemotherapeutics. For the combination with radiotherapy a clinical phase I trial is about to start in esophageal cancer patients. Testing of those kinds of hypoxia specific drugs can be done very efficiently in the so-called 'window of opportunity trials'. After a first hypoxia PET scan which determines the hypoxic fraction present at start of the treatment, a single dose of the hypoxia targeting agent, in this case $\mathrm{TH}-302$, should be given as a monotherapy. One week later a second hypoxia scan can than determine the effect on the hypoxic fraction and determining whether the patient responses to the drug in order to decide the specific treatment in this patient should be continued. After this the regular treatment, including the additional drug, 
can be started. This would be a very cost and patient effective way of testing new hypoxia targeting drugs.

Overall this thesis contains several research projects with a clinical relevance. CAIX imaging and targeting needs additional pre-clinical research before it will be applied in the clinical. However, hypoxia PET imaging is already in clinical trials and for the combination treatment of $\mathrm{TH}-302$ and radiotherapy a clinical phase I trial is about to start. 
Acknowledgements / Dankwoord 
En dan is het bijna zo ver, het enige dat nog rest in dit boekje is een dankwoord. Een beetje een droevig moment omdat er een einde komt aan mijn tijd bij Maastro en ik afscheid ga nemen van veel mensen, maar tegelijkertijd ook een moment om terug te kijken op een mooie periode met vele goede herinneringen. Via deze weg wil ik iedereen bedanken die op welke manier dan ook heeft bijgedragen aan dit boekje. Een aantal mensen wil ik graag in het bijzonder bedanken.

Beste Philippe, bedankt dat je mij de mogelijkheid hebt gegeven om een PhD te doen. Ik waardeer de verscheidenheid aan projecten binnen de (m)CAT groep en de translationele benadering wat leidt tot een echt van 'bench to bedside' proces.

Beste Ludwig, een speciaal dankjewel voor jou. Toen ik 6,5 jaar geleden als junior student bij jou stage kwam lopen had ik niet gedacht dat het zou eindigen met een PhD. Ik was je eerste PhD student en zeker in het begin was dit voor ons alle twee een uitdaging. Ik waardeer je passie voor wetenschap en je oneindige drive om experimenten tot een goed einde te brengen, dag en nacht! Bedankt voor je vertrouwen in mij en alle kansen die ik heb gekregen om mezelf te ontwikkelen tot waar ik nu ben. Ik wens je veel geluk in de toekomst!

Graag wil ik de leden van de leescommissie, Prof dr. F.C.S. Ramaekers, Prof dr. R.P. Coppes, Prof dr. M.J. Daemen, Prof dr. F. Mottaghy, Dr. K.M.A. Rouschop, bedanken voor hun tijd om mijn proefschrift te lezen en beoordelen.

Thanks to all the co-authors. Thank you all for your contribution and scientific input.

Een dankjewel voor de mensen van het CPV, Richard, Saskia, Clarice, Rick, Harry, voor alle goede zorgen en betrokkenheid bij de experimenten.

Ook de mensen van het RNL/SBE, Sandra, Martine, Pascal, wil ik graag bedanken. Jullie makte het altijd weer mogelijk om onze experimenten op een goede en doordachte manier uit te voeren.

Nucleaire geneeskunde, Ivo, Matthias en Maartje, ik keek altijd uit naar jullie telefoontje, wat betekende dat ik uit de startblokken kon en mocht beginnen aan een nieuw experiment. Het was altijd gezellig om even een praatje met jullie te maken. 
Voor alle radioactieve labelingen van de tracers wil ik de VU bedanken. Dankjewel dat ik altijd op jullie kon rekenen als ik weer een levering HX4 nodig had, zelfs als dit meerdere keren per week was!

I would like to thank Threshold Pharmaceuticals for providing us with TH-302 and allowing us to study this drug in combination with radiotherapy. A special thanks to Charles and Jessica for the collaboration and all the scientific input. Katrin, thank you for giving me the opportunity to visit Threshold, it was great to meet everyone!

Voor alle ontspannen uurtjes muziek maken wil ik graag The ProTones bedanken, Lars, Erik, Rianne, Simone, Jørgen, Hubert, Jolanda, Jean, Rob en Ludwig, dankjulliewel voor de gezellige repetities en optredens.

Ook wil ik enkele mensen uit de kliniek bedanken.

Rianne, dankjewel voor de administratieve ondersteuning. Voor alle ondersteuning tijdens het scannen en bestralen wil ik graag de mensen van de PET/CT en de planning bedanken.

Karen en Wouter voor alle hulp en input vanuit het klinische perspectief. Karen, dankjewel dat je altijd tijd maakte voor het analyseren van de beeldjes, altijd met een kritische blik en goede input.

En natuurlijk alle mensen uit de kliniek voor de gezellige Lab vs Kliniek uitjes!

Een speciaal woordje van dank ook voor mijn twee paranimfen, Maud en Rianne.

Lieve Maud, jij was er al vanaf het moment dat ik stage kwam lopen en al snel werden we kantoormaatjes op de $6^{\mathrm{e}}$ verdieping en later weer op de $3^{\mathrm{e}}$. Bij jou kan ik altijd terecht voor goed advies, al dan niet wetenschappelijk (:). Dankjewel voor de vele gezellige momentjes, zonder jou was het lang niet zo leuk geweest!

Lieve Rianne, net als ik begon ook jij als student bij Maastro-lab, al gingen we pas echt samen werken toen je bij de mCAT groep kwam. Ik zal nooit vergeten dat je belde toen je een hele clonogene had laten vallen. Toen je de proefdiercursus ging volgen begon de pret pas echt! Ratjes knuffelen :-). Dankjewel voor al je hulp en je vrolijke, energieke aanwezigheid!

A big thank you to Maastro-lab. A lot has changed over the years, some people left, a lot of new people came; Thank you all for the scientific discussions, friendly environment, coffee breaks and all the fun moments. 
Carla, wat zou het lab zijn zonder jouw vriendelijke aanwezigheid. Dankjewel dat ik met iedere administratieve vraag altijd bij jou terecht kon.

Natasja, toen Ludwig tijdens mijn stage vroeg of ik mee wilde helpen met een proefdierstudie heb ik even getwijfeld, maar jullie hebben mij kunnen overtuigen om dit wel te doen en hier ben ik heel dankbaar voor. Jij hebt me vanaf het begin veel vaardigheden bijgebracht en daarna hebben we met veel plezier samengewerkt op verschillende studies. Dankjewel!

Roger en Marco, als studenten zijn we samen bij Maastro begonnen en hoewel we ieder op een ander onderwerp zaten was er toch een soort van verbondenheid. Sanaz you joined Maastro soon after we all started our PhD and you were also part of this 'generation'. I am really happy that we could share this $\mathrm{PhD}$ experience and it is good to see that we are all finishing up and moving on to the next stage in our careers, all over the world. I wish all of you all the best for the future!

Of course a thank you to all members of the mCAT group, Kranthi, Marike, Simon, Nicolle, Raymon, Ala, Linda, Natasja and Rianne, you are fantastic people and I really enjoyed working with you. Marike, Raymon en Linda, mijn kantoorgenootjes, dank jullie wel voor de gezelligheid op kantoor.

Milou, bedankt voor je inzet en gezelligheid op het lab. En natuurlijk Milou en Barry voor de fietstochtjes die we hebben gemaakt :)

All people that are or were part of Maasto-lab over the past years; Marc, Jan, Ludwig, Kasper, Carla, Natasja, Kim S, Kim P, Rianne, Hanneke, Lydie, Arjan, Paolo, Ala, Barry, Linda, An, Roger, Tom, Marco, Sanaz, Nicolle, Kranthi, Simon, Marike, Raymon, Venus, Eloy, Tessa, Alexy, Maud, Twan, Chantal, Caroline, Nele, Nejla, Ruchi, Frank, Daniela, Janneke. Thank you all for the great time!

Mijn lieve vriendinnetjes wil ik bedanken voor alle afleiding en ontspanning. Jeanou, het is altijd gezellig om met jou bij te kletsen als ik weer eens terug ben in Ospel.

Laura en Nienke, ook al zie ik jullie niet zo heel vaak, het voelt altijd weer als vanouds als ik jullie zie. Ik hoop dat we samen nog veel kerstmarkten gaan bezoeken (;) Mirelle, bedankt voor de gezellige avonden samen koken, thee drinken en kletsen over ons PhD-studenten leventje.

Mirte, Monique en Elsemiek, zonder jullie was mijn leventje in Maastricht bij lange na niet hetzelfde geweest! Sinds onze introductie bij Saurus hebben we veel dingen samen meegemaakt. Dank jullie wel voor de ontelbare etentjes, kopjes thee, glaasjes wijn, slappe lach momentjes, sauna bezoekjes, stapavonden, uitstapjes en natuurlijk 
onze vakanties van dicht bij huis tot iets verder weg! Misschien is het juist wel omdat we alle vier anders zijn dat onze combinatie zo fantastisch werkt :) Ik hoop nog heel veel tijd met jullie door te brengen!

Tot slot wil ik graag mijn familie bedanken voor de belangstelling in mijn werk. Hopelijk heb ik enigszins duidelijk kunnen maken wat ik de afgelopen jaren heb gedaan. Door jullie kijk op de wetenschap werd alles zo nu en dan weer in perspectief geplaatst.

Henny, Ger, Tom en Joyce, bedankt voor het warme welkom in jullie familie. Het is altijd gezellig om naar Brabant te komen.

Fieke, Bas en Bram, en Anne, Bart en Amber, bedankt voor alle gezelligheid en steun de afgelopen jaren. Fieke en Anne, wat is het fijn om zulke lieve zusjes als jullie te hebben! Bas en Bart jullie zorgen samen met Ruud voor het mannelijke tegenwicht als we met z'n alle bij elkaar zijn, wat soms wel nodig is. En natuurlijk super leuk om alle kleine aanwinsten in de familie te mogen verwelkomen :;

Lieve mama, het is altijd heerlijk om weer even naar Ospel te komen en lekker thuis te zijn. Bij jou kan ik altijd terecht voor goed advies, of het nu om een bak recept gaat of over complexere zaken. Dankjewel dat je er altijd voor me bent!

Lieve Papa, ik denk nog vaak aan je, je gevoel voor humor en je positieve, nuchtere kijk op het leven. Zonder jou was dit boekje lang niet zo mooi geweest!

Lieve Ruud, dankjewel voor je steun, begrip, geduld, vrolijkheid en liefde de afgelopen jaren. Ook al zijn we niet altijd samen, toch sta je altijd voor me klaar. Ik kijk enorm uit naar een mooie toekomst samen met jou! 



\section{Curriculum Vitae}


Sarah Peeters was born on February $17^{\text {th }} 1987$ in Weert, the Netherlands. She was raised in the small village of Ospel where she spent her childhood and went to primary school. In 1999 she started high school VWO at Bisschoppelijk College in Weert where she graduated in 2005. In that same year she started the study Molecular Life Sciences at the University in Maastricht. After obtaining her bachelor's degree in 2008, she started the Master Molecular Life Sciences; Clinical Molecular Sciences. During this period she performed two internships at the department of Radiation Oncology (MaastRO), both involving cancer research and the role of CAIX in therapy resistance and malignant behavior of the tumor. In 2010 she finished the Master program and started as a PhD student at Maastricht University, department of Radiation Oncology (MaastRO). Her research involved studying the hypoxic tumor microenvironment with the focus on hypoxia imaging and targeting. The results of her research are presented in this thesis. 


\section{List of publications}


Trani D*, Yaromina A*, Dubois L, Granzier M, Peeters SGJA, Biemans R, et al. Preclinical assessment of efficacy of radiation dose painting based on intratumoral FDGPET uptake. In preparation

Peeters SGJA, Zegers CM, Biemans R, Lieuwes NG, van Stiphout RG, Yaromina A, et al. $\mathrm{TH}-302$ in combination with radiotherapy enhances the therapeutic outcome and is associated with pretreatment $\left[{ }^{18} \mathrm{~F}\right] \mathrm{HX} 4$ hypoxia PET imaging. Clin Cancer Res. 2015 In Press

Peeters SGJA, Dubois L, Lieuwes NG, Laan D, Mooijer M, Schuit RC, et al. [ ${ }^{18}$ F]VM4-037 MicroPET Imaging and Biodistribution of Two In Vivo CAIX-Expressing Tumor Models. Mol Imaging Biol. 2015 In Press

Peeters SGJA*, Zegers CM*, Yaromina A, van Elmpt W, Dubois L, Lambin P. Current pre-clinical and clinical applications of hypoxia PET imaging using 2-nitroimidazoles. Q J Nucl Med Mol Imaging. 2015;59:39-57.

Peeters SGJA, Zegers CM, Lieuwes NG, van Elmpt W, Eriksson J, van Dongen GA, et al. A Comparative Study of the Hypoxia PET Tracers $\left[{ }^{18} \mathrm{~F}\right] \mathrm{HX} 4,\left[{ }^{18} \mathrm{~F}\right] \mathrm{FAZA}$, and $\left[{ }^{18} \mathrm{~F}\right] \mathrm{FMISO}$ in a Preclinical Tumor Model. Int J Radiat Oncol Biol Phys. 2015;91:351-9.

Zegers C, Peeters S, Dubois L, Lambin P. Niet-invasieve PET/CT beeldvorming voor het detecteren en kwantificeren van tumor hypoxie. Oncology News International 2014

Dubois L, Peeters SGJA*, van Kuijk SJ*, Yaromina A, Lieuwes NG, Saraya R, et al. Targeting carbonic anhydrase IX by nitroimidazole based sulfamides enhances the therapeutic effect of tumor irradiation: a new concept of dual targeting drugs. Radiother Oncol. 2013;108:523-8.

Peeters S, Lambin P, Dubois L. Noninvasive hypoxia imaging. In Tumour hypoxia: molecular mechanisms and clinical implications, Pastorekova S, Kopacek J, editors. 2012 Peter Lang GmbH and Veda, SAS Publishing house, p 397-428

Zegers C, Reymen B, Buijsen J, van Stiphout R, Peeters S, Lammering G, Hoebers F, Troost E, van Baardwijk A, Oellers M, van Elmpt W, Lambin P. De bijdrage van PETbeeldvorming in radiotherapie. Het voorbeeld van long en rectumkanker. Kankerbreed 2012 
Dubois L, Peeters S, Lieuwes NG, Geusens N, Thiry A, Wigfield S, et al. Specific inhibition of carbonic anhydrase IX activity enhances the in vivo therapeutic effect of tumor irradiation. Radiother Oncol. 2011;99:424-31.

* indicates equal contribution 
D

$\varnothing$

$\nabla$

$\nabla$

$\Delta$

$\nabla$

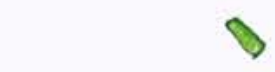

$\vartheta$

$\diamond$

$\nabla$

$\nabla$

D

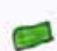

$D$

0

$\Delta$

4

D

a

$\triangleright$

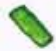

